\title{
Quantification of Back- End Nuclear Fuel Cycle Metrics Uncertainties Due to Cross Sections
}

\author{
Tracy E. Stover, Jr.
}

November 2007

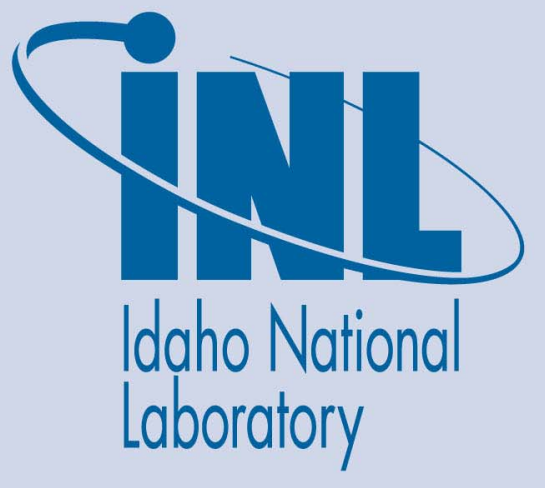

The INL is a U.S. Department of Energy National Laboratory operated by Battelle Energy Alliance 
INL/EXT-07-13592

\title{
Quantification of Back-End Nuclear Fuel Cycle Metrics Uncertainties Due to Cross Sections
}

\author{
Tracy E. Stover, Jr. \\ A Thesis Submitted to the Graduate Faculty of North Carolina State University in \\ Partial Fulfillment of the Requirements for the Degree of Master of Science in \\ Nuclear Engineering \\ Mary Lou Dunzik-Gougar, LDRD Principle Investigator ${ }^{1}$ \\ Dr. Paul Turinsky, LDRD Co-Investigator ${ }^{2}$ \\ ${ }^{1}$ Idaho National Laboratory \\ ${ }^{2}$ North Carolina State University
}

November 2007

Idaho National Laboratory

Idaho Falls, Idaho 83415

Prepared for the

U.S. Department of Energy

Through the INL LDRD Program

Under DOE Idaho Operations Office

Contract DE-AC07-05ID14517 


\begin{abstract}
This work examines uncertainties in the back end fuel cycle metrics of isotopic composition, decay heat, radioactivity, and radiotoxicity. Most advanced fuel cycle scenarios, including the ones represented in this work, are limited by one or more of these metrics, so that quantification of them becomes of great importance in order to optimize or select one of these scenarios. Uncertainty quantification, in this work, is performed by propagating cross-section covariance data, and later number density covariance data, through a reactor physics and depletion code sequence. Propagation of uncertainty is performed primarily via the Efficient Subspace Method (ESM). ESM decomposes the covariance data into singular pairs and perturbs input data along independent directions of the uncertainty and only for the most significant values of that uncertainty. Results of these perturbations being collected, ESM directly calculates the covariance of the observed output posteriori. By exploiting the rank deficient nature of the uncertainty data, ESM works more efficiently than traditional stochastic sampling, but is shown to produce equivalent results. ESM is beneficial for very detailed models with large amounts of input data that make stochastic sampling impractical.
\end{abstract}

In this study various fuel cycle scenarios are examined. Simplified, representative models of pressurized water reactor (PWR) and boiling water reactor (BWR) fuels composed of both uranium oxide and mixed oxides are examined. These simple models are intended to give a representation of the uncertainty that can be associated with open uranium oxide fuel 
cycles and closed mixed oxide fuel cycles. The simplified models also serve as a demonstration to show that ESM and stochastic sampling produce equivalent results, because these models require minimum computer resources and have amounts of input data small enough such that either method can be quickly implemented and a numerical experiment performed. The simplified models are followed by more rigorous reactor physics and depletion models showing a PWR uranium oxide fuel and various metal fast reactor fuels composed of transuranics. The more rigorous models include multi-group cross sections, multiple burnup steps, neutron transport calculations to update cross sections, and multi-scale multi-physics code sequences to simulate a complete fuel lifetime. Finally, the fast reactor and PWR fuels are combined in a closed fast reactor recycle fuel cycle, and uncertainties on the resulting equilibrium cycle examined. 


\section{Acknowledgements}

I would like to thank my advisor Dr. Paul Turinsky whose enthusiastic

guidance and commanding knowledge of the subject have been crucial to both this work and my post-graduate development. Next, I thank Dr. Hany Abdel-Khalik who has been continuously available to assist me in this work and help me through the many difficulties in the subject matter. I would also like to thank Dr. Masood Iqbal for very helpful conversation and instruction in the use of alternative cross-section processors and depletion codes. In particular I must thank Mr. Matthew Jessee for his vast support in dealing with and understanding the implementation of mathematics involved in this research, and Mr. Ross Hays, Mr. Christopher Briggs, and Mr. Jason Elkins for their thoughtful advice on advanced programming and instruction in the use of various models. 


\section{Table of Contents}

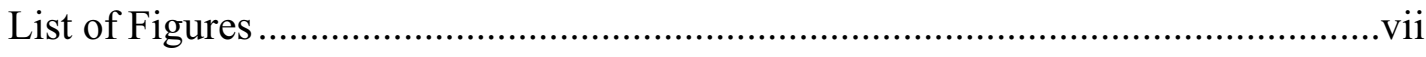

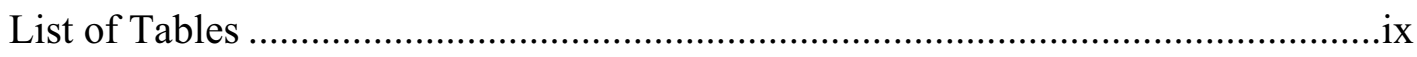

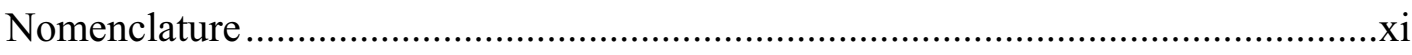

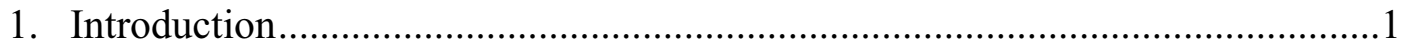

1.1 Importance to the Nuclear Fuel Cycle ...............................................

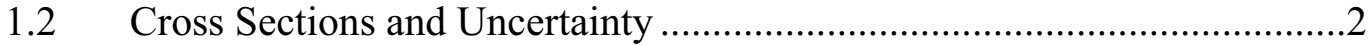

1.3 Review of Uncertainty Propagation Techniques ....................................

1.4 Overview of Computational Modeling Software.....................................8

1.5 Fuel Types and Scenarios of Interest ..................................................11

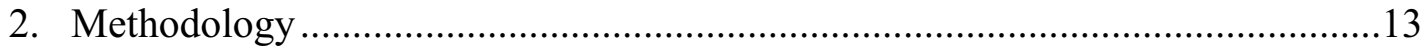

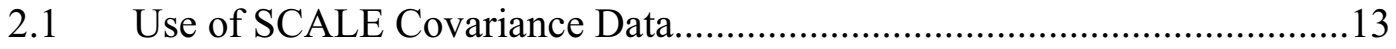

2.2 Use of REBUS Covariance Data ......................................................... 16

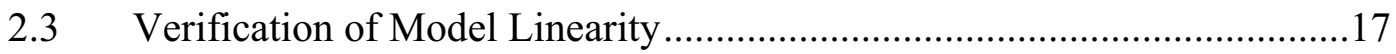

2.4 Implementation of Stochastic Sampling Method.....................................21

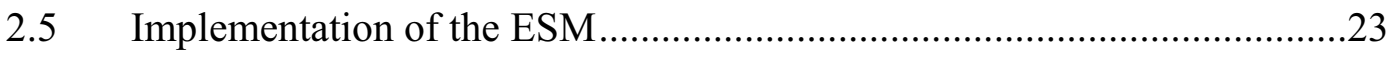

2.6 Computational Models Employed for Each Method ................................26

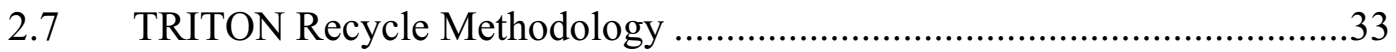

2.8 Statistical Analysis Performed on Results .................................................

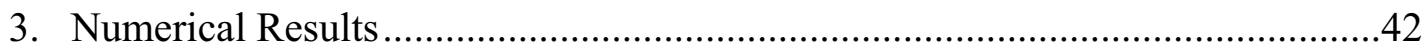

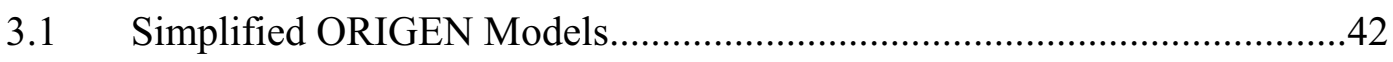

3.1.1 Equivalency of ESM and Stochastic Methods...........................42

3.1.2 PWR Model with UOX Fuel ...................................................44

3.1.3 Typical LWR with UOX Fuel..................................................48

3.1.4 BWR Models with UOX Fuel....................................................49

3.1.5 PWR Models with MOX Fuels .....................................................52

3.1.6 Comparison of Results, Simplified ORIGEN Models ...................55

3.1.7 A Brief Experiment with Operational Uncertainties .....................62

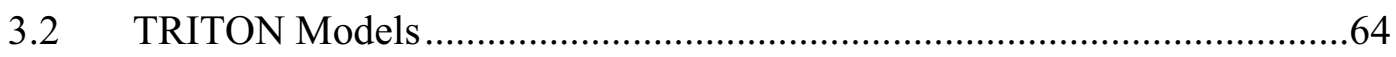

3.2.1 PWR Model in TRITON with UOX Fuel..................................64

3.2.2 Fast Reactor Models with Transuranic Fuels..............................75

3.2.3 Fast Reactor Model with Transuranic Fuel and Recycling.............79 
3.2.4 Comparison of Results, TRITON Models ...................................83

3.3 REBUS Fast Reactor Equilibrium Model..........................................89

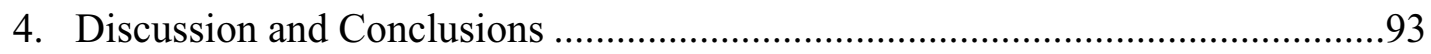

4.1 Discussion of the Use of ESM in this Study .........................................93

4.2 Discussion Concerning the Results of the Models ...................................93

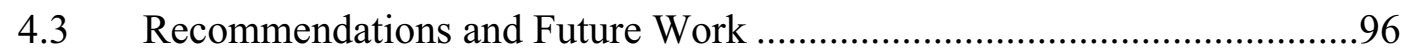

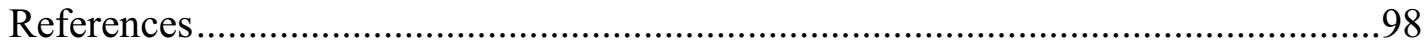

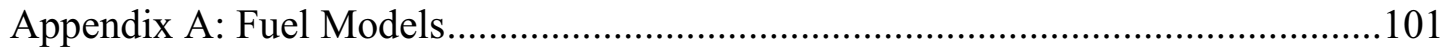

I. The Typical LWR Model Using a UOX Fuel.......................................101

II. The PWR Model Using a UOX Fuel ....................................................101

III. The BWR Model Using a UOX Fuel..................................................104

IV. The PWR Separation Model Using a UOX Fuel ...................................107

V. The PWR Model Using a MOX Fuel ...................................................108

VI. The PWR Model Using a MOX Fuel with Impurities ............................111

VII. The PWR Model Using TRITON .....................................................115

VIII. TRITON FR Models Using Actinide Fuels .........................................118

IX. REBUS Fast Reactor Model with Actinide Fuel and Recycle ................126

Appendix B: Graphical Verification of Model Linearity ...................................... 140

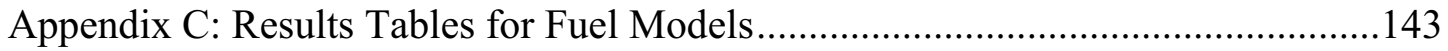




\section{$\underline{\text { List of Figures }}$}

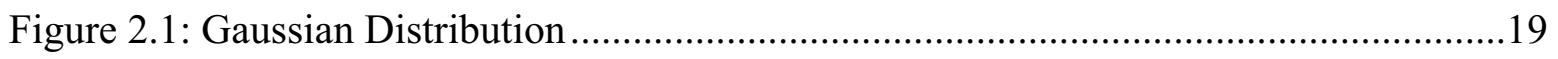

Figure 2.2: Flux Spectra for PWR Models. ...................................................................28

Figure 2.3: Flux Spectra for BWR Models.................................................................28

Figure 3.1: Decay heat comparison of stochastic and ESM sampling methods..................58

Figure 3.2: Radioactivity comparison of stochastic and ESM sampling methods. ..............58

Figure 3.3: Decay heat comparison of SCALE provided data and SAS2H updated data....59

Figure 3.4: Radioactivity, SCALE provided data and SAS2H updated data.......................59

Figure 3.5: Decay heat comparison for BWR fuels........................................................60

Figure 3.6: Radioactivity Comparison for BWR fuels. ...............................................60

Figure 3.7: Decay heat comparison of UOX and MOX fuels.........................................61

Figure 3.8: Radioactivity comparison for UOX and MOX fuels......................................61

Figure 3.9: Decay heat uncertainty of key isotopes due to various sources. ......................62

Figure 3.10: Radioactivity uncertainty in key isotopes due to various sources...................63

Figure 3.11: Decay heat comparison of simple ORIGEN and TRITON models. ................85

Figure 3.12: Radioactivity comparison of simple ORIGEN and TRITON models..............85

Figure 3.13: Decay heat comparison of three fast reactor fuels. .....................................86

Figure 3.14: Radioactivity comparison for three fast reactor fuels. .................................86

Figure 3.15: Decay heat comparison of once through and recycled fast reactor fuels. .........87

Figure 3.16: Radioactivity comparison of once through and recycled fast reactor fuels. .....87

Figure 3.17: k-effective uncertainty due to recycled isotopics uncertainties only. ...............88

Figure 3.18: k-effective uncertainty due to cross sections and recycled

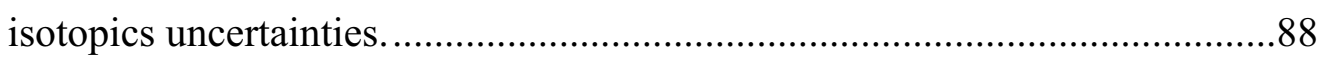

Figure 3.19: Decay heat comparison of REBUS and TRITON Models............................92

Figure 3.20: Radioactivity comparison of REBUS and TRITON Models. ........................92

Figure A.II.1: SAS2H Model, PWR, 4.5 w/o and 40 GWD/MTU ....................................102

Figure A.II.2: ORIGEN Model, PWR, 4.5 w/o and 40 GWD/MTU .................................103

Figure A.III.1: SAS2H Model, BWR, 4.5 w/o and 40 GWD/MTU ...................................105

Figure A.III.2: ORIGEN Model, BWR, 4.5 w/o and 40 GWD/MTU ...............................106

Figure A.V.1: SAS2H Model, MOX fuel in PWR, 50 GWD/MTHM ...............................109

Figure A.V.2: ORIGEN Model, MOX fuel in PWR, 50 GWD/MTHM ...........................110

Figure A.VI.1: SAS2H Model, MOX with impurities fuel in PWR, 50 GWD/MTHM .......112 
Figure A.VI.2: ORIGEN Model, MOX with impurities fuel in PWR, 50 GWD/MTHM.....113

Figure A. VII.1: TRITON Model, PWR, 4.5 w/o and 48 GWD/MTU .................................115

Figure A. VIII.1: TRITON Model, CR=1.05 ………………………………………........119

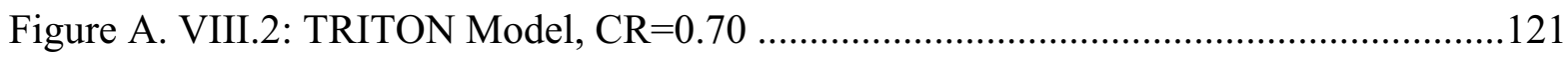

Figure A. VIII.3: TRITON Model, CR=0.25 ............................................................123

Figure A.IX.1: REBUS Equilibrium Model, CR = 0.77..................................................126

Figure B.1: Nuclide Output Discharge Mass Linearity ………..........................................141

Figure B.1: Sampling Output Nuclide Discharge Mass Distributions....................................142 


\section{List of Tables}

Table 2.1: Listing of Considered Nuclides and Reactions in SCALE Library.....................16

Table 2.2: Listing of Considered Nuclides and Reactions in REBUS Library.....................17

Table 2.3: Brief Summary of Fuel Types Examined Using SAS2H + ORIGEN ................27

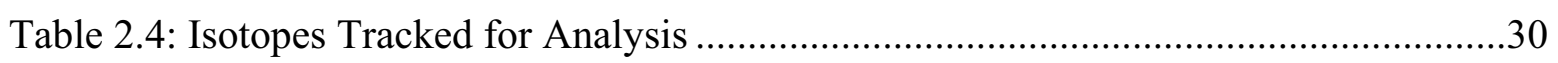

Table 2.5: Brief Summary of Fuel Types Examined Using TRITON ................................31

Table 2.6: Specific values, per nuclide, for metrics of interest.........................................39

Table 3.1: Comparison of isotopic uncertainties from the two methods. .............................43

Table 3.2: Discharge isotopics for the PWR simplified model. ..........................................44

Table 3.3: Masses, with uncertainty, of actinides at 3 decay times. ..................................45

Table 3.4: Comparison of separation at 3 times vs. no separation, first 100047

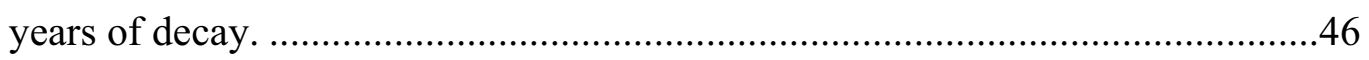

Table 3.5: Comparison of separation at 3 times vs. no separation, $2500-$

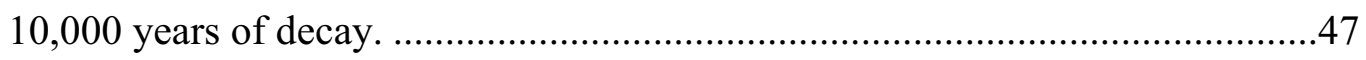

Table 3.6: Discharge isotopics for typical LWR simplified model. ...................................48

Table 3.7: Isotopics uncertainties for typical LWR simplified model ...............................49

Table 3.8: Discharge isotopics for BWR fuel burned at $0 \%$ void. ....................................50

Table 3.9: Discharge isotopics for BWR fuel burned at 35\% void. .....................................50

Table 3.10: Discharge isotopics for BWR fuel burned at 50\% void. ................................51

Table 3.11: Discharge isotopics for BWR fuel burned at $65 \%$ void. .................................51

Table 3.12: Isotopics uncertainties for BWR models. ......................................................52

Table 3.13: Discharge isotopics for the "clean" MOX fuel. .............................................54

Table 3.14: Discharge isotopics for MOX fuel with impurities. .......................................54

Table 3.15: Isotopic uncertainties for MOX fuels. ....................................................55

Table 3.16: Comparison of isotopics between models. ................................................65

Table 3.17: 40 GWD/MTU Discharge Isotopics ..............................................................66

Table 3.18: 48 GWD/MTU Discharge Isotopics .........................................................66

Table 3.19: Resulting standard deviations from both models.........................................67

Table 3.20: Change in flux as burnup increases. .............................................................. 70

Table 3.21: Change in 1-group cross section uncertainty due to flux change. ....................71

Table 3.22: Comparing discharge isotopic uncertainties for various flux updates...............72

Table 3.23: Change in Am-243 capture cross section due to resonance treatment. .............7 
Table 3.24: Fuel composition data for fast reactor models. .77

Table 3.25: Fuel geometry and power data for fast reactor models..................................77

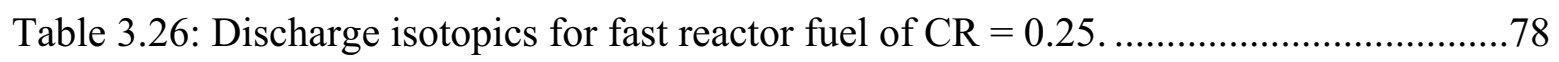

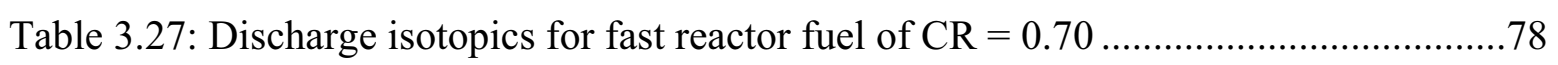

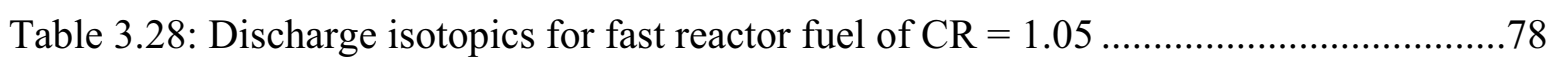

Table 3.29: Relative isotopic uncertainties for fast reactor fuels........................................79

Table 3.30: Comparison of fuel composition properties for once through and

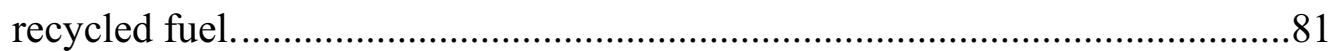

Table 3.31: Discharge isotopics for equilibrium recycled fuel........................................81

Table 3.32: Discharged isotopics uncertainties originating from recycled isotopics

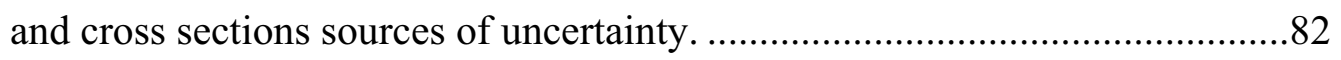

Table 3.33: Operating Parameters for REBUS model......................................................91

Table 3.34: Discharge Isotopics for REBUS model. ..........................................................91

Table 3.35: Isotopc Uncertainties for REBUS model..................................................91

Table A.IV.1: Isotopics of discharged UOX fuel at 5, 10, and 25 years after irradiation.....108

Table A.VIII.1: Fast Reactor Fuel Composition Data, by conversion ratio ........................118

Table A. VIII.2: Cladding Composition Data.................................................................118

Table A. VIII.3: Operating Conditions and Geometry Data...............................................118

Table C.1: Results table for the PWR model using ESM for uncertainty. ...........................144

Table C.2: Results table for PWR fuel in the simplified model. ...................................... 145

Table C.3: Results table for typical LWR fuel simplified model. .....................................146

Table C.4: Results table for BWR fuel burned at $0 \%$ void...............................................147

Table C.5: Results table for BWR fuel burned at 35\% void.............................................. 148

Table C.6: Results table BWR fuel burned at 50\% void. .................................................149

Table C.7: Results table for BWR fuel burned at $65 \%$ void............................................... 150

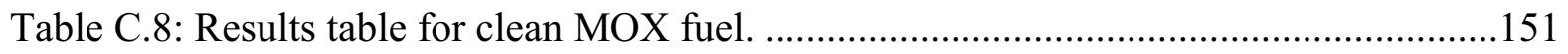

Table C.9: Results table for MOX fuel with impurities......................................................152

Table C.10: Results table for TRITON PWR model, 48 GWD/MTU................................153

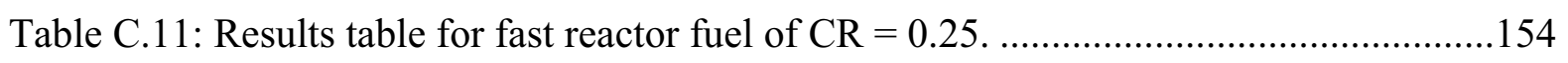

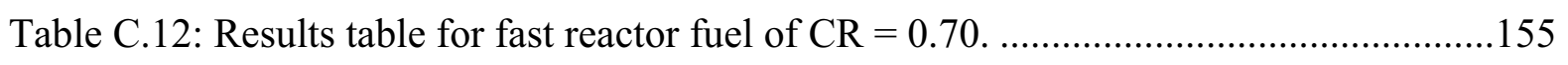

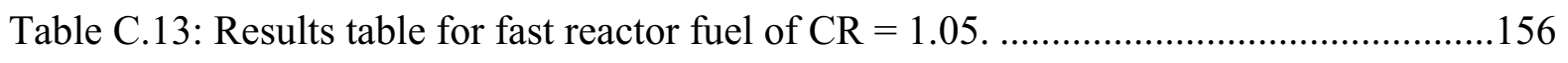

Table C.14: Results table for equilibrium recycled fast reactor fuel of $\mathrm{CR}=0.70 \ldots \ldots \ldots \ldots .157$

Table C.15: Results table for REBUS equilibrium recycled fast reactor fuel. .....................158 


\section{Nomenclature}

ALWR - Advanced Light Water Reactor

BOC - Beginning of Cycle

BOL - Beginning of Life

BWR - Boiling Water Reactor

BU - Burnup

CR - Conversion Ratio

ENDF - Evaluated Nuclear Data File

EOC - End of cycle

EOL - End of Life

ESM - Efficient Subspace Method(s)

FP - Fission Product(s)

FR - Fast Reactor

GWD - Giga Watt Days

LWR - Light Water Reactor

MOX - Mixed Oxide

MTHM - Metric Ton Heavy Metal

MTIHM - Metric Ton Initial Heavy Metal

MTU - Metric Ton Uranium

PWR - Pressurized Water Reactor

TRU - Transuranic(s)

UOX - Uranium Oxide

w/o - Weight Percent 


\section{QUANTIFICATION OF BACK-END NUCLEAR FUEL CYCLE METRICS UNCERTAINTIES DUE TO CROSS SECTIONS 1. Introduction}

\subsection{Importance to the Nuclear Fuel Cycle}

Over the next several years, policy makers will be assessing the deployment of various components of the_nuclear fuel_cycle, e.g. the Yucca Mountain_repository, reprocessing plants, new reactors, etc. This research will be conducted in conjunction with the SINEMA (Simulation Institute for Nuclear Energy Modeling and Analysis) project headed by Idaho National Laboratory, which aims to produce a computational tool to be provided to policy makers for the assessment and comparison of various fuel cycle scenarios [1]. The objective of this work is to develop uncertainty propagation techniques to assess the affect of certain design and operation parameters on back-end fuel cycle metrics that are of key importance in various fuel cycle scenarios. Comparing two fuel cycles might be irrelevant if the uncertainty in a key metric between them overlaps.

Key metrics will hereinafter be defined as anything that is a limiting factor for the technology or facilities which are deployed in the current nuclear fuel cycle or may be deployed in future advanced fuel cycle scenarios. The nearest future deployment seems to be the spent fuel repository to be located at Yucca Mountain, Nevada. The repository's capacity is currently limited by the heat produced by the decay of the spent fuel such that the temperature between the repository tunnels remains below the local boiling temperature of water. Heat load is dominated by fission products in the first 1500 years, when peak heat production occurs and by minor actinides thereinafter [2][3]. In the very distant future the waste packages are assumed to fail and the metric of concern is then what material is released, i.e. isotopic inventory and the radiotoxicity of the material released to the biosphere. It has been suggested that implementing a so-called advanced fuel cycle that 
includes reprocessing of spent nuclear fuel could extend the lifetime of the repository by reusing fissile material and reclassifying inert material that would otherwise fill the repository quickly in the once through fuel cycle [4]. A good example is that greater than $95 \%$ of spent uranium oxide fuel is U-238, a low level waste that is safe enough to store somewhere other than the repository if separated out [5]. When considering the reprocessing of spent fuel for a mixed oxide fuel for a light water reactor, or an actinide fuel for a fast reactor, the concerns become radioactivity of the fuel, which facilities must contain, and the inventory of material which can be extracted from the fuel at the time of separation. Convenience of physical properties requires only the examination of the uncertainty that arises in isotopic inventories since heat, radioactivity, and radiotoxicity are linearly proportional to mass. Apart from the significant economical and political challenges of implementing advanced fuel cycles or operating a repository at all, e.g. high cost of reprocessing and poor public opinion [6], the nature of engineering requires designs to be built around safety margins which are limited by the metrics discussed above. Reducing uncertainty not only allows for a better evaluation of fuel cycles but also more economical and efficient designs of the associated infrastructure.

\subsection{Cross Sections and Uncertainty}

Reaction cross-sections, as part of Evaluated Nuclear Data File (ENDF) [7], provide a large amount of information that is essential to any nuclear calculation, e.g. the models that predict the behavior and operation of nuclear reactors and the resulting spent fuel. Since the aim of this work is to develop a generalized uncertainty propagation technique for nuclear models that demand large input data sets and produce large output parameter data sets, crosssections' uncertainty is the source that will be examined. The uncertainty cross sections 
contribute to the output parameters of discharged isotopic masses, decay heat load, radioactivity and radiotoxicity are the back-end nuclear fuel cycle metrics that are analyzed herein. Since the evaluation of these data is continuously being updated, emphasis is placed on their uncertainties - variances and covariances - with that data also made available in conjunction with the cross-sections themselves. The problem is augmented by the complex nature of cross-sections, measured as a function of the kinetic energy of the neutrons that are causing the reactions. Homogenization, or the averaging of a cross-section over a fixed energy range and/or spatial region, is often implemented to reduce the computational burden. The level of homogenization varies depending upon the application, ranging from hundreds of pieces of data for simple depletion, to millions of data pieces for precise in-core calculations. Considering that every material charged to, or created in, a reactor has many cross-sections for many different reaction types, even when represented by only one energy group, spatially homogenized over the entire core, the volume of uncertainty data is still large and propagating its affect on various metrics is a daunting task.

\subsection{Review of Uncertainty Propagation Techniques}

Uncertainty data allows uncertainty models to be applied and propagated through crucial parameters for evaluating the design system in question, such as reactor operation and the nuclear fuel cycle as a whole. "Propagating uncertainties is a non-trivial task because of the computational complexity often associated with the various modeling stages of the fuel cycles, and the size and type of different sources of uncertainties." [8] It is also beneficial to recognize that modeling uncertainties can be introduced through the numerical approximations that are typically found in models of complex systems, but for this study the 
focus is on those sources of uncertainty that are inputs to the model, particularly crosssections input to a nuclear physics model.

The most basic analytical method is to perturb an input by some value and observe how the output is affected. While this approach efficiently arrives at a direct sensitivity of a model to an individual parameter, the investigator will usually only examine a few parameters due to the time requirements. Case in point is the work of E. Schneider [10] who introduced set perturbations into a few key cross sections and modeled the response of discharge isotopics to those perturbations. When considering huge volumes of input data such as thousands of cross-sections coupled with long CPU run-times of complex, multiphysic models, this method is very tedious and time consuming.

The classic approach to the uncertainty analysis of nuclear systems is the use of adjoint solutions that arrive at the sensitivity of a metric to all input parameters [9]. While the change in the metric to any change in that particular parameter is now known, the drawback is that $m$ metrics will require $m$ adjoint solutions [11]. If one follows this process to obtain sensitivity coefficients, $\mathrm{S}$, for many parameters, for example $\mathrm{R}$ cross sections, $\mathrm{S}_{\mathrm{R}}$ one arrives at a so-called sensitivity matrix $\overline{\overline{S_{R}}}$. Note that here and throughout the remainder of this document, variables shown with a single bar are assumed to be vectors and variables with a double bar are assumed to be matrices. The uncertainty matrix of a metric to this set of parameters is easily obtained by multiplying the sensitivity matrix by the covariance matrix of the parameters by the transpose of the sensitivity matrix [11][12][13], producing what is sometimes called the "sandwich" equation. This classical approach has been studied and repeated, and consistently yields reliable and verified results. The work of H. Aliberti, et. al. uses this approach to evaluate the uncertainty of reactor and fuel cycle parameters, e.g. 
reactivity, decay heat, etc., in regards to cross-sections and is a valuable source with which to compare the results of this work.

The process can be very time consuming from a computational viewpoint because, every metric must have an adjoint solution and a set of sensitivity parameters evaluated. H. AbdelKhalik of North Carolina State University has recently developed the Efficient Subspace Method (ESM) which approximates the behavior of a large, rank deficient matrix, such as the cross-section covariance matrix or the sensitivity matrix, in an effort to make computations more efficient [14]. ESM works most efficiently when the input data and the number of metrics of interest to be observed are both large. ESM also requires the problem to be illconditioned, as are many complex system problems. ESM can be implemented in existing models, but requires linear algebra operations to be applied via pre- and post- processors. In addition to its use for propagating cross-sections uncertainties, ESM has been harnessed for performing adaptive simulation of reactor core calculations. Adaptive simulation is an inverse theory approach that adjusts cross-sections to enhance the agreement between the measured and code-predicted core observables of interest, e.g. core power distribution, and core reactivity. Adaptive simulation is currently the focus of various research projects at NC State.

Another method of uncertainty propagation is the so-called forward perturbation method, which can either be deterministic or stochastic in nature [11]. The deterministic approach works best when the input data field is small because this method determines sensitivity by input data perturbation one piece at a time [15]. Because the input data set for cross-sections can be very large, this approach was not considered. Alternatively, the stochastic approach can be confidently used for a larger input data set and works well when 
the amount of output data is large [15]. This method uses a Monte Carlo sampling (random or Latin-Hypercube [16]) of the total input data skewed by the data probability distributions. Many samples of inputs are run with existing models and probability distributions of output are determined directly from the results [11][15]. A study of the convergence of the distributions is often necessary to determine the number of samples needed to assure confidence in a specific problem.

Directly sampling the probability distribution is ideal only when the input parameters are independent [15]. In the case of covariance data for a large number of parameters input to a complex multi-scale model, two main issues arise: 1) in using random input samples to calculate outputs that are functions of many variables, some sample sets could be linearly dependent, i.e. the output could be approximated by a linear combination of previous samples, increasing the number of samples required because essentially the same sample is being repeated, and 2) covariance is defined as the expected variance of one random variable with respect to another random variable [17], which means that the probability distributions of input parameters are correlated, and that simply sampling a distribution of one parameter does not take into account its variance due to another. In the realm of linear algebra, covariances exist as the off diagonal elements of the covariance matrix and variances are the diagonal elements.

If the model is linear, both of these issues can be avoided by a single adjustment to the forward method. To account for correlations and to ensure that each set of samples is linearly independent, the covariance matrix is processed by singular value decomposition into eigenvalues and corresponding eigenvectors. The eigenvalues which are derived from the covariance matrix are used in the probability distributions and the eigenvectors, by 
definition, are linearly independent. The samples used as input are a combination of samples from each of the eigen-pairs where the square root of the eigenvalue is the standard deviation of the sample [18]. When H. Kawano, et. al., used this procedure, it was applied only to the multi-group covariance matrix of the Pu-239 fission cross section. The resulting affects on criticality were subsequently examined and compared to a benchmark experiment. While fission of Pu-239 is very important for both uranium and mixed oxide fuels, as well as nuclear weapons, it is still just one reaction among many. When the covariance matrix is very sparse, this method yields another benefit for the analysis in that the eigenvectors of a sparse matrix will contain one element that is very close to 1 and the other elements will be very small. Thus, if one perturbs along only a single eigenvector at a time, the perturbation can be traced back to a single cross-section since one would have received the majority of the perturbation along that eigenvector. This is used to determine which cross-sections contribute most to the resulting uncertainty in the output.

In reviewing the methods available, both ESM and stochastic forward perturbation using the eigen-pair approach and random sampling show promise for such a problem as set forth in this work. It will be shown later that the models used in this work are nearly linear and converge after a reasonable number of samples to justify using either approach. In development of the propagation techniques in this work, the stochastic perturbation approach was used on simple LWR fuel models, namely uranium and mixed oxide fuels. Due to the fast execution time of simplified and somewhat crude models of these fuels and the linear algebra processors required for ESM, both of which will be addressed in Section 2, this appeared a prudent choice. The benefit of this simplified model is that one can compare the traditional stochastic method to the newly developed ESM. A validation experiment for 
ESM, implemented within the simple model, shows that both methods produced equivalent results but that the stochastic method required less mathematical manipulation. When a much more detailed realistic fuel model is needed, however, e.g. many burnup steps in a neutron transport model using multi-group cross section data, stochastic methods become impractical and the use of the ESM becomes necessary. Such a model is the standard in practical fuel analysis and is also needed when the simplified models failed to provide needed resolution and linearity when examining fast reactor fuels. Due to the fact that the two approaches were determined to be equivalent, the move to this method, was made with confidence.

\subsection{Overview of Computational Modeling Software}

As already stated, the techniques developed in this work are implemented in preexisting fuel cycle models. Computational modeling programs are cornerstones of the nuclear industry since full scale experiments are often not a pursuable approach. The preexisting models chosen for this study are the SCALE 5.0 software package available from Oak Ridge National Laboratory, specifically, the ORIGEN depletion code, the SAS2H sequence, and the TRITON sequence; and, the REBUS 3.0 code from Argonne National Laboratory. Qualifications of the SCALE package include verified and validated models of benchmark experiments augmented by package popularity, user-friendliness, and convenient technical support from the developer [19]. Furthermore, the SCALE package also includes a pre-formatted 44-group library containing variance and covariance information for a number of key reactions types and isotopes. The REBUS model, which has also been verified and validated, has a somewhat more difficult input structure, but was specifically designed for fast reactor models [20]. 
ORIGEN is a time-dependent point-depletion analysis code that can track changes in concentrations of a large number of isotopes due to nuclear transmutation and radioactive decay. The program uses the matrix exponential expansion method to solve the Bateman depletion equations for any number of discrete points in time. ORIGEN can model nuclear fuel at various stages during the fuel cycle, including irradiation, storage, transportation, etc. ORIGEN operates with various library formats, the two most common being a card image library and a binary working library. The three-group card image library must be supplied by the user in the required format and include a corresponding three group flux spectrum in the ORIGEN input deck. SCALE is distributed with a three-group card image library, and its corresponding flux spectrum that is representative of a typical light water reactor. The typical flux spectrum is also available in 44-group and 238-group representations. The library type most often used is an AMPX formatted binary library. The master libraries containing basic ENDF data in 1-, 3-, 44-, and 238- groups are included with the SCALE package. Because SCALE is a multi-physics program, there are drivers and programs that can update the master library to create a problem-specific working library that is usable in ORIGEN. When ORIGEN uses a binary library, the cross sections applied are in either onegroup or three-group values that are representative of the specific problem that the working library was created for. This allows ORIGEN to execute very quickly and elminates the need to input a specially formatted card-image library or a fuel specific flux spectrum, which is already accounted for in the new cross-sections. [21]

The SAS2H sequence uses various codes within SCALE to produce a detailed model of a fuel assembly. SAS2H is a coupled one-dimensional depletion and shielding analysis sequence. SAS2H is designed to create a 1-D model of a specific fuel type and then track 
various parameters -- reactivity, isotopics, dose rates in storage, etc. -- through the life of the fuel. The user supplies a fuel composition, geometry, power and decay history, and optionally, a storage cask description for disposal dose analysis. Problem specific, burnup dependent cross-sections are derived using two separate lattice cell models in a pseudo 2-D model that utilizes 1-D neutron transport modeling. The process also produces problemdependent flux spectra in the same number and ranges of groups as the master library input to SAS2H. SAS2H uses the ORIGEN code to do all of its depletion analysis both for the incore depletion and out-of-core decay. While SAS2H was mainly designed to model light water reactor and research reactor fuels, it can also be used to create a crude fast reactor model if given the fuel composition and geometry for such a reactor. [22]

The TRITON sequence is also another all inclusive depletion analysis, like the SAS2H routine. Unlike SAS2H, however, TRITON solves the transport equation in a 2dimensional geometry. TRITON is particularly used for modeling single fuel assemblies or individual Wigner cells, the latter of which will be used in this work. TRITON must be given buffer region input as it does not automatically account for non-fuel holes in the lattice like SAS2H. The biggest drawback is that TRITON was developed intentionally for commercial reactors whose fuel is by standard in a square lattice. While TRITON can model any number of polygon geometries within a given domain, the outer domain is forced to be rectangular, which is effective for square unit cells but lacks the resolution and proper moderator modeling abilities for other geometries, for example, a hexagonal cell for a fast reactor fuel. This work recognizes this shortcoming of the model and acknowledges that the results will not be absolutely accurate because of it. In its defense, TRITON is a much more 
detailed model than SAS2H and overcomes some modeling inadequacies of SAS2H while maintaining all the analysis abilities. [23]

REBUS is used in the latter part of this study to compare the fast reactor results from TRITON, since the fast reactor models examined were created at Argonne using this code. Also, the many group cross section library and associated covariance matrix for REBUS is based upon a sodium cooled fast reactor flux spectrum, whereas the data available in the SCALE package is based upon a light water thermal reactor flux spectrum. REBUS was used since it has the unique ability to recycle fuel, using both reprocessing plants and external sources, and iteratively find some equilibrium fuel composition to meet operating parameters and cycle energy requirements, while using the available recycle feed. As used in this study, REBUS incorporates the DIF3D diffusion theory code utilizing the finite difference option. Hexagonal-z geometry for the core is modeled, with each hexagon representing a fuel assembly with homogenized cross sections employed. The drawback to REBUS is that a few-group covariance matrix did not exist a priori as it did with the SCALE package. Thanks to the work of Dr. Masood Iqbal and Dr. Hany Abdel-Khalik, a 15-group covariance matrix [24] for key reaction types and isotopes was created specifically for REBUS at North Carolina State University using the Argonne cross section processing code $M^{2} 2$ [25]. Dr. Hany Abdel-Khalik also implemented the efficient subspace method (ESM) of uncertainty propagation in REBUS.

\subsection{Fuel Types and Scenarios of Interest}

The most logical place to begin the analysis of uncertainty in various fuel types is to first analyze the fuel of the current reactor fleet deployed in the U.S. - low enriched uranium oxide fuel. Care is taken to select, directly or similarly from other studies, fuel types that 
represent an actual equilibrium cycle fuel or a fuel for a predicted equilibrium cycle, i.e. not a specialized fuel designed for start-up cores or demonstration experiments. For the current reactor designs in this study, that fuel is a $4.5 \mathrm{w} / \mathrm{o}$ uranium oxide fuel burned to 40 GWD/MTU modeled first by the typical light water reactor information provided with SCALE, and then in both a pressurized water reactor and a boiling water reactor of various void fractions as modeled by SAS2H. To consider an advanced fuel cycle in the advent of a reprocessing infrastructure being considered in the U.S., mixed oxide and fast reactor fuels are also considered. Models include an ALWR MOX containing plutonium and uranium, a mixed oxide fuel with neptunium and americium impurities, and three fast reactor fuels, of various conversion ratios, made up of spent light water reactor fuel to burn off minor actinides. Finally, an experiment is conducted to demonstrate the effects of accumulating uncertainty in the input isotopics themselves as fuel is recycled in the fast reactor case. The former single pass fast reactor models are examined in TRITON and the latter fast reactor recycle scenario will be modeled both TRITON and REBUS. The fast reactor and its corresponding fuel types are modeled after Argonne's Advanced Burner Test Reactor [26]. 


\section{Methodology}

\subsection{Use of SCALE Covariance Data}

The SCALE 5.0 package is distributed with two 44-group covariance libraries, based on a light water thermal reactor flux spectrum, that contain information for approximately 700 nuclide-reaction pairs for many key isotopes. A full listing of all available data is too lengthy for this document but the reader is referred to the manual describing the library [31]. Effort is taken, through assumptions and model limits, to reduce this volume of data both to fit the input needed for models and to reduce the computational effort needed to implement the chosen uncertainty propagation technique. Unexpectedly, one of the assumptions made so the data will fit the ORIGEN code, actually expands the volume of information.

The covariance library containing information for most nuclide-reaction pairs is chosen as the data source for this work. The first reduction in data is to examine only the reactions that are important to reactor calculation for depletion analysis, and the only reactions ORIGEN uses -- neutron capture and fission. Those reactions in particular are: $(n, \gamma),(n, p),(n, \alpha),($ fission$),(n, 2 n)$, and $(n, 3 n)$. The result is that covariance data for 701 nuclide-reaction pairs is reduced to 116 pairs by removing the cross-sections that are not of interest to depletion. For the simplified models, the perturbations are introduced into ORIGEN as the cross sections are read from the library, whereas with the more rigorous TRITON model, perturbations are made directly in the master cross section library before it is used by the code. When coupling a binary library to ORIGEN, generated by SAS2H as described later, the simplified model directly uses one-group cross-sections that are ideally representative of the specific problem. With this restriction on input data, the 44-group 
neutron flux spectrum generated by SAS2H is used to collapse the 44-group covariance data to one-group values, instantly reducing the volume of data by a factor of 44 solely so that its affects can be applied directly to ORIGEN. With the exception of the typical library examined, which was prepared a priori by Oak Ridge, the flux spectra are generated for each simplified fuel/reactor examined in this work and the beginning of life total flux was chosen as a representative spectrum to be used for the collapse. This topic will be addressed again when discussing the TRITON results. For TRITON, perturbations obtained from the 44group covariance library can be introduced directly into the 44-group master cross section library given as input to the code since both are of the same group structure. This eliminates all the pre-processing discussed above for the simplified models.

The covariance library contains data for ten materials in elemental form rather than the isotope specific reaction that ORIGEN uses. While this is of no consequence to TRITON which recognizes elemental forms and deals with them internally, the simplified models that use only ORIGEN for sampling need nuclide specific values. With this in mind, the data for those ten elements - magnesium, silicon, potassium, chromium, iron, nickel, copper, zirconium, hafnium and lead - is assumed to apply equally to isotopes of each element which are included in the cross section library. The result is the expansion of the data to a final value of 223 nuclide-reaction pairs that are considered in this work. Table 2.1 lists the nuclide-reaction pairs, using asterisks $\left(^{*}\right)$ to indicate data that were expanded from the elemental form and crosses $(\dagger)$ indicating pairs that have off-diagonal covariance data. 


\begin{tabular}{|c|c|c|c|c|c|}
\hline Nuclide & Reaction(s) & Nuclide & Reaction(s) & Nuclide & Reaction(s) \\
\hline $\mathrm{H}-1$ & $(\mathrm{n}, \gamma)$ & Co-59 & $\begin{array}{l}(\mathrm{n}, 2 \mathrm{n}),(\mathrm{n}, \gamma), \\
(\mathrm{n}, \alpha)\end{array}$ & Eu-153 & $(\mathrm{n}, \gamma)$ \\
\hline Li-6 & $\begin{array}{l}(\mathrm{n}, \gamma) \text { (not used in } \\
\text { ORIGEN) }\end{array}$ & Ni-58* & $\begin{array}{l}(\mathrm{n}, \gamma),(\mathrm{n}, \mathrm{p}),(\mathrm{n}, \alpha) \\
(\mathrm{n}, 2 \mathrm{n})\end{array}$ & Eu-154 & $(\mathrm{n}, \gamma)$ \\
\hline $\mathrm{Li}-7$ & $(\mathrm{n}, \gamma)$ & Ni-59* & $\begin{array}{l}(\mathrm{n}, \gamma),(\mathrm{n}, \mathrm{p}),(\mathrm{n}, \alpha) \\
(\mathrm{n}, 2 \mathrm{n})\end{array}$ & Eu-155 & $(n, \gamma)$ \\
\hline B-10 ${ }^{\dagger}$ & $(n, p)$ & Ni-60* & $\begin{array}{l}(\mathrm{n}, \gamma),(\mathrm{n}, \mathrm{p}),(\mathrm{n}, \alpha) \\
(\mathrm{n}, 2 \mathrm{n})\end{array}$ & Gd-154 & $(\mathrm{n}, \gamma)$ \\
\hline $\mathrm{C}-12$ & $(\mathrm{n}, \gamma),(\mathrm{n}, \mathrm{p}),(\mathrm{n}, \alpha)$ & Ni-61* & $\begin{array}{l}(\mathrm{n}, \gamma),(\mathrm{n}, \mathrm{p}),(\mathrm{n}, \alpha) \\
(\mathrm{n}, 2 \mathrm{n})\end{array}$ & Gd-155 & $(\mathrm{n}, \gamma)$ \\
\hline $\mathrm{N}-14$ & $(\mathrm{n}, \gamma),(\mathrm{n}, \mathrm{p}),(\mathrm{n}, \alpha)$ & $\mathrm{Ni}-62^{*}$ & $\begin{array}{l}(\mathrm{n}, \gamma),(\mathrm{n}, \mathrm{p}),(\mathrm{n}, \alpha) \\
(\mathrm{n}, 2 \mathrm{n})\end{array}$ & Gd-156 & $(\mathrm{n}, \gamma)$ \\
\hline O-16 & $(n, p),(n, \alpha)$ & Ni-63* & $\begin{array}{l}(\mathrm{n}, \gamma),(\mathrm{n}, \mathrm{p}),(\mathrm{n}, \alpha) \\
(\mathrm{n}, 2 \mathrm{n})\end{array}$ & Gd-157 & $(\mathrm{n}, \gamma)$ \\
\hline F-19 & $(\mathrm{n}, \gamma),(\mathrm{n}, \mathrm{p}),(\mathrm{n}, \alpha)$ & Ni-64* & $\begin{array}{l}(\mathrm{n}, \gamma),(\mathrm{n}, \mathrm{p}),(\mathrm{n}, \alpha) \\
(\mathrm{n}, 2 \mathrm{n})\end{array}$ & Hf-174* & $(\mathrm{n}, \gamma)$ \\
\hline $\mathrm{Na}-23$ & $\begin{array}{l}(\mathrm{n}, \gamma),(\mathrm{n}, \mathrm{p}),(\mathrm{n}, \mathrm{\alpha}) \\
(\mathrm{n}, 2 \mathrm{n})\end{array}$ & $\mathrm{Ni}-65^{*}$ & $\begin{array}{l}(\mathrm{n}, \gamma),(\mathrm{n}, \mathrm{p}),(\mathrm{n}, \alpha) \\
(\mathrm{n}, 2 \mathrm{n})\end{array}$ & Hf-175* & $(n, \gamma)$ \\
\hline Mg-24* & $(\mathrm{n}, \gamma)$ & Ni-66* & $\begin{array}{l}(\mathrm{n}, \gamma),(\mathrm{n}, \mathrm{p}),(\mathrm{n}, \mathrm{\alpha}) \\
(\mathrm{n}, 2 \mathrm{n})\end{array}$ & Hf-176* & $(\mathrm{n}, \gamma)$ \\
\hline $\mathrm{Mg}-25^{*}$ & $(\mathrm{n}, \gamma)$ & $\mathrm{Cu}-63^{*}$ & $(\mathrm{n}, \gamma)$ & Hf-177* & $(\mathrm{n}, \gamma)$ \\
\hline Mg-26* & $(n, \gamma)$ & Cu-64* & $(\mathrm{n}, \gamma)$ & Hf-178* & $(n, \gamma)$ \\
\hline Mg-27* & $(\mathrm{n}, \gamma)$ & $\mathrm{Cu}-65^{*}$ & $(\mathrm{n}, \gamma)$ & Hf-179* & $(\mathrm{n}, \gamma)$ \\
\hline Mg-28* & $(\mathrm{n}, \gamma)$ & Cu-66* & $(\mathrm{n}, \gamma)$ & Hf-180* & $(\mathrm{n}, \gamma)$ \\
\hline Al-27 & $\begin{array}{l}(\mathrm{n}, \gamma),(\mathrm{n}, \mathrm{p}),(\mathrm{n}, \alpha) \\
(\mathrm{n}, 2 \mathrm{n})\end{array}$ & Cu-67* & $(\mathrm{n}, \gamma)$ & Hf-181* & $(\mathrm{n}, \gamma)$ \\
\hline $\mathrm{Si}-28 *$ & $(n, p),(n, \alpha)$ & $\mathrm{Zr}-89^{*}$ & $(\mathrm{n}, \gamma)$ & Hf-182* & $(\mathrm{n}, \gamma)$ \\
\hline Si-29* & $(n, p),(n, \alpha)$ & Zr-90* & $(\mathrm{n}, \gamma)$ & Au-197 & $(\mathrm{n}, \gamma)$ \\
\hline Si-30* & $(n, p),(n, \alpha)$ & $\mathrm{Zr}-91^{*}$ & $(\mathrm{n}, \gamma)$ & $\mathrm{Pb}-204 *$ & $(\mathrm{n}, 2 \mathrm{n}),(\mathrm{n}, 3 \mathrm{n}),(\mathrm{n}, \gamma)$ \\
\hline Si-31* & $(n, p),(n, \alpha)$ & Zr-92* & $(\mathrm{n}, \gamma)$ & $\mathrm{Pb}-205^{*}$ & $(\mathrm{n}, 2 \mathrm{n}),(\mathrm{n}, 3 \mathrm{n}),(\mathrm{n}, \gamma)$ \\
\hline Si-32* & $(n, p),(n, \alpha)$ & Zr-93* & $(\mathrm{n}, \gamma)$ & $\mathrm{Pb}-206^{*}$ & $(\mathrm{n}, 2 \mathrm{n}),(\mathrm{n}, 3 \mathrm{n}),(\mathrm{n}, \gamma)$ \\
\hline $\mathrm{K}-39 *$ & $(\mathrm{n}, \gamma)$ & Zr-94* & $(\mathrm{n}, \gamma)$ & $\mathrm{Pb}-207^{*}$ & $(\mathrm{n}, 2 \mathrm{n}),(\mathrm{n}, 3 \mathrm{n}),(\mathrm{n}, \gamma)$ \\
\hline $\mathrm{K}-40 *$ & $(\mathrm{n}, \gamma)$ & Zr-95* & $(\mathrm{n}, \gamma)$ & $\mathrm{Pb}-208^{*}$ & $(\mathrm{n}, 2 \mathrm{n}),(\mathrm{n}, 3 \mathrm{n}),(\mathrm{n}, \gamma)$ \\
\hline $\mathrm{K}-41 *$ & $(\mathrm{n}, \gamma)$ & Zr-96* & $(\mathrm{n}, \gamma)$ & $\mathrm{Pb}-209^{*}$ & $(\mathrm{n}, 2 \mathrm{n}),(\mathrm{n}, 3 \mathrm{n}),(\mathrm{n}, \gamma)$ \\
\hline K-42* & $(\mathrm{n}, \gamma)$ & $\mathrm{Zr}-97^{*}$ & $(\mathrm{n}, \gamma)$ & Th-232 & $(\mathrm{n}, \gamma),($ fission$)$ \\
\hline K-43* & $(\mathrm{n}, \gamma)$ & Mo-95 & $(\mathrm{n}, \gamma)$ & U-233 & $(\mathrm{n}, \gamma),($ fission $)$ \\
\hline Cr-50* & $\begin{array}{l}(\mathrm{n}, \gamma),(\mathrm{n}, \mathrm{p}),(\mathrm{n}, \alpha) \\
(\mathrm{n}, 2 \mathrm{n}),(\mathrm{n} 3 \mathrm{n})\end{array}$ & Tc-99 & $(\mathrm{n}, \gamma)$ & U-234 & $(\mathrm{n}, \gamma),($ fission$)$ \\
\hline Cr-51* & $\begin{array}{l}(\mathrm{n}, \gamma),(\mathrm{n}, \mathrm{p}),(\mathrm{n}, \alpha) \\
(\mathrm{n}, 2 \mathrm{n}),(\mathrm{n} 3 \mathrm{n})\end{array}$ & Ru-101 & $(\mathrm{n}, \gamma)$ & $\mathrm{U}-235^{\dagger}$ & $(\mathrm{n}, \gamma),($ fission $)$ \\
\hline Cr-52* & $\begin{array}{l}(\mathrm{n}, \gamma),(\mathrm{n}, \mathrm{p}),(\mathrm{n}, \alpha) \\
(\mathrm{n}, 2 \mathrm{n}),(\mathrm{n} 3 \mathrm{n})\end{array}$ & Rh-103 & $(\mathrm{n}, \gamma)$ & U-236 & $(\mathrm{n}, \gamma),($ fission$)$ \\
\hline Cr-53* & $\begin{array}{l}(\mathrm{n}, \gamma),(\mathrm{n}, \mathrm{p}),(\mathrm{n}, \alpha) \\
(\mathrm{n}, 2 \mathrm{n}),(\mathrm{n} 3 \mathrm{n})\end{array}$ & Ag-109 & $(\mathrm{n}, \gamma)$ & $\mathrm{U}-238^{\dagger}$ & $\begin{array}{l}(\mathrm{n}, \gamma),(\text { fission}), \\
(\mathrm{n}, 2 \mathrm{n}),(\mathrm{n} 3 \mathrm{n})\end{array}$ \\
\hline
\end{tabular}




\begin{tabular}{|c|c|c|c|c|c|}
\hline Cr-54* & $\begin{array}{l}(\mathrm{n}, \gamma),(\mathrm{n}, \mathrm{p}),(\mathrm{n}, \alpha) \\
(\mathrm{n}, 2 \mathrm{n}),(\mathrm{n} 3 \mathrm{n})\end{array}$ & In-115 & $(\mathrm{n}, \gamma)$ & $\mathrm{Np}-237$ & (fission) \\
\hline Cr-55* & $\begin{array}{l}(\mathrm{n}, \gamma),(\mathrm{n}, \mathrm{p}),(\mathrm{n}, \alpha) \\
(\mathrm{n}, 2 \mathrm{n}),(\mathrm{n} 3 \mathrm{n})\end{array}$ & Cs-133 & $(\mathrm{n}, \gamma)$ & $\mathrm{Pu}-238$ & $(\mathrm{n}, \gamma),($ fission$)$ \\
\hline Mn-55 & $(\mathrm{n}, 2 \mathrm{n})$ & $\mathrm{Nd}-143$ & $(\mathrm{n}, \gamma)$ & $\mathrm{Pu}-239^{\dagger}$ & $(\mathrm{n}, \gamma),($ fission $)$ \\
\hline Fe-54* & $\begin{array}{l}(\mathrm{n}, \gamma),(\mathrm{n}, \mathrm{p}),(\mathrm{n}, \alpha) \\
(\mathrm{n}, 2 \mathrm{n})\end{array}$ & $\mathrm{Nd}-145$ & $(\mathrm{n}, \gamma)$ & $\mathrm{Pu}-240^{\dagger}$ & $(\mathrm{n}, \gamma),($ fission $)$ \\
\hline $\mathrm{Fe}-55^{*}$ & $\begin{array}{l}(\mathrm{n}, \gamma),(\mathrm{n}, \mathrm{p}),(\mathrm{n}, \alpha) \\
(\mathrm{n}, 2 \mathrm{n})\end{array}$ & Sm-147 & $(\mathrm{n}, \gamma)$ & $\mathrm{Pu}-241^{\dagger}$ & $(\mathrm{n}, \gamma),($ fission$)$ \\
\hline Fe-56* & $\begin{array}{l}(\mathrm{n}, \gamma),(\mathrm{n}, \mathrm{p}),(\mathrm{n}, \alpha) \\
(\mathrm{n}, 2 \mathrm{n})\end{array}$ & Sm-159 & $(\mathrm{n}, \gamma)$ & $\mathrm{Pu}-242^{\dagger}$ & $\begin{array}{l}(\mathrm{n}, \gamma),(\text { fission}), \\
(\mathrm{n}, 2 \mathrm{n}),(\mathrm{n} 3 \mathrm{n})\end{array}$ \\
\hline Fe-57* & $\begin{array}{l}(\mathrm{n}, \gamma),(\mathrm{n}, \mathrm{p}),(\mathrm{n}, \alpha) \\
(\mathrm{n}, 2 \mathrm{n})\end{array}$ & Sm-150 & $(\mathrm{n}, \gamma)$ & $\mathrm{Am}-241^{\dagger}$ & $(\mathrm{n}, \gamma),($ fission$)$ \\
\hline Fe-58* & $\begin{array}{l}(\mathrm{n}, \gamma),(\mathrm{n}, \mathrm{p}),(\mathrm{n}, \alpha) \\
(\mathrm{n}, 2 \mathrm{n})\end{array}$ & Sm-151 & $(n, \gamma)$ & Am-243 & $(\mathrm{n}, \gamma),($ fission $)$ \\
\hline Fe-59* & $\begin{array}{l}(\mathrm{n}, \gamma),(\mathrm{n}, \mathrm{p}),(\mathrm{n}, \alpha) \\
(\mathrm{n}, 2 \mathrm{n})\end{array}$ & Sm-152 & $(n, \gamma)$ & & \\
\hline
\end{tabular}

Table 2.1: Listing of Considered Nuclides and Reactions in SCALE Library.

\subsection{Use of REBUS Covariance Data}

The 15-group covariance library, developed for a sodium cooled fast reactor, pertains explicitly to the 15-group cross section files which are used in the REBUS model. Due to their specific nature, and the fact that they will be directly used to create perturbations in the REBUS fast reactor models, no modification or simplifications are necessary. The 101 reaction types for twenty isotopes included in this library are available from Brookhaven National Laboratory [24]. The nuclides and reactions represented appear in Table 2.2. 


\begin{tabular}{|c|c|c|c|c|c|}
\hline Nuclide & Reaction(s) & Nuclide & Reaction(s) & Nuclide & Reaction(s) \\
\hline \multirow[t]{2}{*}{$\mathrm{Cr}-52$} & $(\mathrm{n}, \gamma),(\mathrm{n}, \mathrm{el}),\left(\mathrm{n}, \mathrm{n}^{\prime}\right)$ & Np-237 & $\begin{array}{l}(\mathrm{n}, \gamma),(\text { fission}), \\
(\mathrm{n}, \mathrm{el})\end{array}$ & $\begin{array}{l}\text { Am- } \\
242 m\end{array}$ & $\begin{array}{l}(\mathrm{n}, \gamma),(\text { fission}), \\
(\mathrm{n}, \mathrm{el})\end{array}$ \\
\hline & $(\mathrm{n}, 2 \mathrm{n})$ & & $\begin{array}{l}(\mathrm{n}, 2 \mathrm{n}),\left(\mathrm{n}, \mathrm{n}^{\prime}\right),(\mathrm{v}- \\
\text { bar) }\end{array}$ & & $\begin{array}{l}(\mathrm{n}, 2 \mathrm{n}),\left(\mathrm{n}, \mathrm{n}^{\prime}\right),(\mathrm{v}- \\
\text { bar })\end{array}$ \\
\hline \multirow[t]{2}{*}{ Fe-56* } & $(\mathrm{n}, \gamma),(\mathrm{n}, \mathrm{el}),\left(\mathrm{n}, \mathrm{n}^{\prime}\right)$ & $\mathrm{Pu}-238$ & $\begin{array}{l}(\mathrm{n}, \gamma),(\text { fission}), \\
(\mathrm{n}, \mathrm{el})\end{array}$ & $\begin{array}{l}\text { Am- } \\
243\end{array}$ & $\begin{array}{l}(\mathrm{n}, \gamma),(\text { fission}), \\
(\mathrm{n}, \mathrm{el})\end{array}$ \\
\hline & $(\mathrm{n}, 2 \mathrm{n})$ & & $(\mathrm{n}, 2 \mathrm{n}),\left(\mathrm{n}, \mathrm{n}^{\prime}\right)$ & & $\begin{array}{l}(\mathrm{n}, 2 \mathrm{n}),\left(\mathrm{n}, \mathrm{n}^{\prime}\right),(\mathrm{v}- \\
\text { bar })\end{array}$ \\
\hline \multirow[t]{2}{*}{$\mathrm{Ni}-58^{*}$} & $(\mathrm{n}, \gamma),(\mathrm{n}, \mathrm{el}),\left(\mathrm{n}, \mathrm{n}^{\prime}\right)$ & $\mathrm{Pu}-239$ & $\begin{array}{l}(\mathrm{n}, \gamma),(\text { fission}), \\
(\mathrm{n}, \mathrm{el})\end{array}$ & $\mathrm{Cm}-242$ & $\begin{array}{l}(\mathrm{n}, \gamma),(\text { fission}), \\
(\mathrm{n}, \mathrm{el})\end{array}$ \\
\hline & $(\mathrm{n}, 2 \mathrm{n})$ & & $\begin{array}{l}(\mathrm{n}, 2 \mathrm{n}),\left(\mathrm{n}, \mathrm{n}^{\prime}\right),(\mathrm{v}- \\
\text { bar })\end{array}$ & & $(\mathrm{n}, 2 \mathrm{n}),\left(\mathrm{n}, \mathrm{n}^{\prime}\right)$ \\
\hline \multirow[t]{2}{*}{ U-234 } & $(\mathrm{n}, \gamma),($ fission$),(\mathrm{n}, \mathrm{el})$ & $\mathrm{Pu}-240$ & $\begin{array}{l}(\mathrm{n}, \gamma),(\text { fission}) \\
(\mathrm{n}, \mathrm{el})\end{array}$ & $\mathrm{Cm}-243$ & $\begin{array}{l}(\mathrm{n}, \gamma),(\text { fission}), \\
(\mathrm{n}, \mathrm{el})\end{array}$ \\
\hline & $(\mathrm{n}, 2 \mathrm{n}),\left(\mathrm{n}, \mathrm{n}^{\prime}\right)$ & & $\begin{array}{l}(\mathrm{n}, 2 \mathrm{n}),\left(\mathrm{n}, \mathrm{n}^{\prime}\right),(\mathrm{v}- \\
\text { bar })\end{array}$ & & $(\mathrm{n}, 2 \mathrm{n}),\left(\mathrm{n}, \mathrm{n}^{\prime}\right)$ \\
\hline \multirow[t]{2}{*}{$\mathrm{U}-235$} & $(v$-bar) & $\mathrm{Pu}-241$ & $\begin{array}{l}(\mathrm{n}, \gamma),(\text { fission}), \\
(\mathrm{n}, \mathrm{el})\end{array}$ & $\mathrm{Cm}-244$ & $\begin{array}{l}(\mathrm{n}, \gamma),(\text { fission}), \\
(\mathrm{n}, \mathrm{el})\end{array}$ \\
\hline & & & $\begin{array}{l}(\mathrm{n}, 2 \mathrm{n}),\left(\mathrm{n}, \mathrm{n}^{\prime}\right),(\mathrm{v}- \\
\text { bar })\end{array}$ & & $(\mathrm{n}, 2 \mathrm{n}),\left(\mathrm{n}, \mathrm{n}^{\prime}\right)$ \\
\hline \multirow[t]{2}{*}{ U-236 } & $(\mathrm{n}, \gamma),($ fission$),(\mathrm{n}, \mathrm{el})$ & $\mathrm{Pu}-242$ & $\begin{array}{l}(\mathrm{n}, \gamma),(\text { fission}), \\
(\mathrm{n}, \mathrm{el})\end{array}$ & $\mathrm{Cm}-245$ & $\begin{array}{l}(\mathrm{n}, \gamma),(\text { fission}), \\
(\mathrm{n}, \mathrm{el})\end{array}$ \\
\hline & $(\mathrm{n}, 2 \mathrm{n}),\left(\mathrm{n}, \mathrm{n}^{\prime}\right)$ & & $(\mathrm{n}, 2 \mathrm{n}),\left(\mathrm{n}, \mathrm{n}^{\prime}\right)$ & & $(\mathrm{n}, 2 \mathrm{n}),\left(\mathrm{n}, \mathrm{n}^{\prime}\right)$ \\
\hline \multirow[t]{2}{*}{ U-238 } & $(\mathrm{n}, \gamma),($ fission$),(\mathrm{n}, \mathrm{el})$ & Am-241 & $\begin{array}{l}(\mathrm{n}, \gamma),(\text { fission}) \\
(\mathrm{n}, \mathrm{el})\end{array}$ & & \\
\hline & $(n, 2 n),\left(n, n^{\prime}\right),(v-b a r)$ & & $\begin{array}{l}(\mathrm{n}, 2 \mathrm{n}),\left(\mathrm{n}, \mathrm{n}^{\prime}\right),(\mathrm{v}- \\
\text { bar })\end{array}$ & & \\
\hline
\end{tabular}

Table 2.2: Listing of Considered Nuclides and Reactions in REBUS Library.

\subsection{Verification of Model Linearity}

As stated in the introduction, before either stochastic forward perturbation or ESM methods are implemented, it is reasonable to check the linearity of the model to be used. Uncertainty propagation can be done by either a Monte Carlo sampling scheme, which can propagate all the moments of the input data, given infinite runs, to build the probability distributions, or a Moments Method, which propagates only selected moments of the distribution [11]. Assuming that the choice between the methods depends on the nature of 
the probability distribution of the input data and the linearity of the model, it is reasonable to spend some time on this topic.

To illustrate this, the definition of the first and second moments are introduced as follows, assuming the probability distribution of input data $y$, is $p(y)$ and $p(y) d y$ is the probability that input data will be between $y$ and $y+d y$ :

$$
\begin{gathered}
p_{1}=\int_{-\infty}^{\infty} y p(y) d y=\mu \\
p_{2}=\int_{-\infty}^{\infty} y^{2} p(y) d y=\sigma^{2}-\mu^{2} \Rightarrow p_{2}-\mu^{2}=\int_{-\infty}^{\infty}(y-\mu)^{2} p(y) d y=\sigma^{2}
\end{gathered}
$$

where $\mu$ is the average of all possible values of the input data, i.e. the mean, and $\sigma^{2}$ describes the average of the squared differences between all possible values and the mean, i.e. the variance. Higher order moments exist with physical and statistical meaning, but since they will not be addressed in this work, further explanation is available in the references [11][17]. These moments are what define a probability distribution and the nature of the distribution determines how many moments are needed for its reconstruction.

The Gaussian distribution depicted in Figure 2.1 is characterized by only the first two moments, mean and variance.

$$
p_{\text {Gauss }}(y)=\frac{1}{\sigma \sqrt{2 \pi}} \exp \left(-\frac{(y-\mu)^{2}}{2 \sigma^{2}}\right)
$$




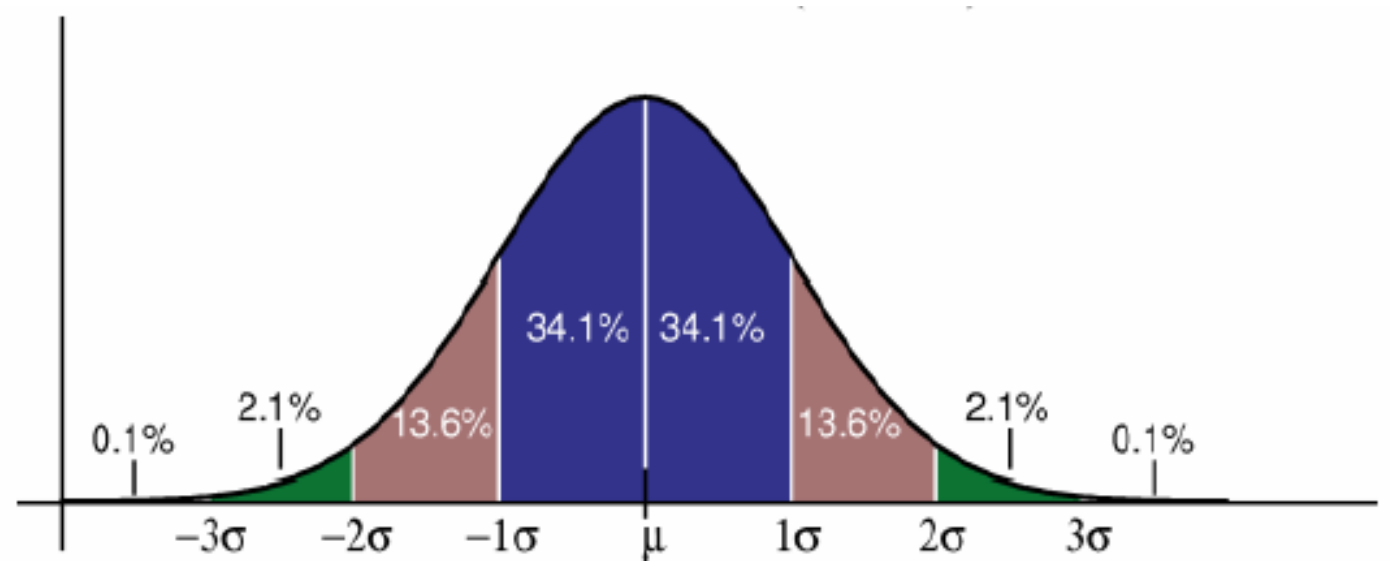

Figure 2.1: Gaussian Distribution

Further, rigorous mathematical proof shows that if a model is linear, a Gaussian input will produce a Gaussian output [23]. The first moment of the output corresponds to the reference output values calculated based on the mean input values. The second moment, variance, is obtained by re-running the model with input data perturbed by an amount proportional to the standard deviation. Most input cross-sections lack information about the second moment and no ENDF library contains information about higher order moments. For this reason it is often assumed cross-sections are normally distributed given a lack of higher order moments. Along with that assumption, this work is based on the observation that the model used, i.e. ORIGEN, which shall be discussed in detail later, is nearly linear over the range of uncertainties of interest.

To study the linearity of the model the following study is conducted. Let the model be defined by an operator, $\Omega$,:

$$
\overline{y_{0}}=\Omega\left(\overline{\sigma_{0}}\right)
$$

where $\overline{\sigma_{0}}$ is a vector of input cross-sections $i=1, \ldots N$ where subscript 0 denotes reference values, and $\overline{y_{0}}$ are calculated isotopics. The model $\Omega$ is judged linear around $\overline{\sigma_{0}}$ if it satisfies the condition: 
Given arbitrary perturbations $\delta \bar{\sigma}_{i}, i=1 \rightarrow N$

Calculate $\delta \bar{y}_{i}=\Omega\left(\bar{\sigma}_{0}+\delta \bar{\sigma}_{i}\right)-\Omega\left(\bar{\sigma}_{0}\right)$, for $i=1 \rightarrow N$

Then, $\Rightarrow \Omega\left(\bar{\sigma}_{0}+\sum_{i=1}^{N} a_{i} \delta \bar{\sigma}_{i}\right)-\Omega\left(\bar{\sigma}_{0}\right)=\sum_{i}^{N} a_{i} \delta \bar{y}_{i}$

for arbitrary $a_{i}$

The physical interpretation is for every cross-section perturbation, $\delta \overline{\sigma_{i}}$, the corresponding

affect $\delta \overline{y_{i}}$ is obtained by running the code with the reference value and then again with

cross-sections perturbed. The code can be run $N$ times with each execution corresponding to a random cross-section perturbation, and then run with cross-sections perturbed by a linear combination of the previous $N$ perturbations, i.e.

$$
\bar{\sigma}=\overline{\sigma_{0}}+\sum_{i=1}^{N} a_{i} \delta \overline{\sigma_{i}}
$$

where $a_{i}$ are arbitrary weights. If the model is linear, the perturbations should be approximately given by a linear combination of the original perturbations $\delta \overline{y_{i}}$, i.e.

$$
\Omega\left(\sigma_{0}+\sum_{i=1}^{N} a_{i} \delta \overline{\sigma_{i}}\right)-\Omega\left(\sigma_{0}\right) \approx \sum_{i}^{N} a_{i} \delta \overline{y_{i}}
$$

The difference between the two approaches is used to qualitatively judge model linearity. It is assumed that the weights $a_{i}$ summed over $N$ equal 1.

This qualitative approach must be applied to all generated outputs. If at any time the output is judged non-linear over a range of uncertainties, in this case within 4 standard deviations of the mean, then the outputs would no longer be Gaussian. It was so determined that the ORIGEN model is nearly linear. Appendix B includes further graphical support by showing 1) linear changes in output isotopics over a range of cross section perturbations, and 2) Gaussian output of samples given Gaussian inputs. 


\subsection{Implementation of Stochastic Sampling Method}

A linear model allows the implementation of either ESM or stochastic perturbation. Before discussing the implementation of the uncertainty propagation method, it is worthwhile to review the structure and origin of the covariance matrix, particularly as it exists in the SCALE library chosen as the data source for the majority of this work. The cross-section covariance matrix is given by:

$$
\overline{\overline{C_{\sigma}}}=\left[\begin{array}{cccc}
\operatorname{Cov}\left(\sigma_{1}, \sigma_{1}\right) & \operatorname{Cov}\left(\sigma_{1}, \sigma_{2}\right) & \cdots & \operatorname{Cov}\left(\sigma_{1}, \sigma_{n}\right) \\
\operatorname{Cov}\left(\sigma_{1}, \sigma_{2}\right) & \operatorname{Cov}\left(\sigma_{2}, \sigma_{2}\right) & \cdots & \vdots \\
\vdots & \cdots & \ddots & \vdots \\
\operatorname{Cov}\left(\sigma_{n}, \sigma_{1}\right) & \cdots & \cdots & \operatorname{Cov}\left(\sigma_{n}, \sigma_{n}\right)
\end{array}\right]
$$

where $\operatorname{Cov}\left(\sigma_{\mathrm{i}}, \sigma_{\mathrm{j}}\right)$ is the absolute covariance between cross-sections $\mathrm{i}$ and $\mathrm{j}$ and is defined by:

$$
\operatorname{Cov}\left(\sigma_{i}, \sigma_{j}\right)=\int_{-\infty}^{\infty}\left(\sigma_{i}-\sigma_{i 0}\right)\left(\sigma_{j}-\sigma_{j 0}\right) p\left(\sigma_{i}, \sigma_{j}\right) d \sigma_{i} d \sigma_{j}
$$

In this notation, subscripts $i$ and $j$ denote isotope, energy group, and reaction type dependence. Since the absolute values of the cross-sections will change for each unique problem, it is not convenient to work with this absolute covariance data. The relative covariance matrix, in which each element is between -1 and 1 , will be useful for simplifying the perturbation method shown later and can be obtained by using:

$$
C_{R i j}=\frac{\operatorname{Cov}\left(\sigma_{i}, \sigma_{j}\right)}{\sigma_{i} \sigma_{j}}
$$

to obtain [30]:

$$
\overline{\overline{C_{R \sigma}}}=\left[\begin{array}{cccc}
C_{R 11} & C_{R 12} & \cdots & C_{R 1 n} \\
C_{R 21} & C_{R 22} & \cdots & \vdots \\
\vdots & \cdots & \ddots & \vdots \\
C_{R n 1} & \cdots & \cdots & C_{R n n}
\end{array}\right]
$$


If given the sensitivity matrix of y with respect to $\sigma, \overline{\overline{S_{R}}}$, the uncertainty in the output parameters, $\overline{\overline{C_{y}}}$, can be evaluated as

$$
\overline{\overline{C_{y}}}=\overline{\overline{S_{R}}} \overline{\overline{C_{R \sigma}}}{\overline{\overline{S_{R}}}}^{T}
$$

In practice, these matrices or their products, are rarely directly constructed, but the effect of this product when using the forward perturbation with eigen-pair approach is evaluated as follows.

The singular value decomposition of $\overline{\overline{C_{R \sigma}}}$ is defined as:

$$
\overline{\overline{C_{R \sigma}}}=\overline{\bar{W}} \overline{\bar{\Sigma}_{\sigma}} \overline{\bar{W}}^{T}
$$

where $\overline{\overline{\Sigma_{\sigma}}}$ is the diagonal matrix of eigenvalues and $\overline{\bar{W}}$ the orthonormal matrix of eigenvectors where $\overline{\bar{W}}=\left[\overline{w_{1}}, \overline{w_{2}}, \ldots, \overline{w_{n}}\right]$ and $\bar{w}_{i}^{T} \overline{w_{j}}=0 \leftrightarrow i \neq j$.

Since this is a stochastic forward perturbation method, a form of Monte Carlo sampling is implemented. Each sample is a perturbation of each cross section, and that perturbation, $\gamma_{\mathrm{i}}$, for cross-section $i$, is defined as follows [18]:

$$
\gamma_{i}=\sum_{j=1}^{n} \xi_{j}\left(\overline{w_{j}}\right)_{i}
$$

where the value $\xi_{\mathrm{j}}$ is a random sample obtained from the eigenvalue $\Sigma_{\mathrm{jj}}$ having the Gaussian distribution defined as:

$$
p\left(\xi_{j}\right) d \xi_{j}=\frac{1}{\sqrt{2 \pi \Sigma_{j}}} \exp \left(\frac{-\xi_{j}^{2}}{2 \Sigma_{j}}\right) d \xi_{j}
$$

Finally, since the covariance data in the matrix $\overline{\overline{C_{R \sigma}}}$ that was decomposed was relative data, any perturbed cross-section, $\sigma_{i}$, is simply: 


$$
\sigma_{i}=\sigma_{i 0}\left(1+\gamma_{i}\right)
$$

where $\sigma_{i 0}$ denotes the unperturbed cross section. Perturbations are introduced thusly for all cross-sections $i=1, \ldots, n$. The matrix decomposition and creation of a set of input perturbations can be done a priori by auxiliary codes developed specifically for this purpose, thus sampling can use the model as a tool to produce perturbed results without modifying the model itself.

\subsection{Implementation of the ESM}

As indicated earlier, ESM methods are a favorable alternative to a stochastic forward perturbation when dealing with a large volume of input data, in this case a cross-section covariance matrix that is sparse and ill-conditioned, as required for ESM. The following section will describe the ESM method in brief but for the most detailed, rigorous, and formal definition, the reader is referred to H. Abdel-Khalik [14].

Consider $n$ input data and $m$ output data derived by using the model $\Omega$. ESM states that for $n$ inputs, at most $n$ runs are required to fully characterize the distributions of the output, as opposed to stochastic methods which typically require a number of samples on the order of $n$. Define $\bar{y}$ as the vector of $\mathrm{m}$ number densities calculated by:

$$
\bar{y}=\overline{\bar{\Omega}}(\bar{\sigma})=\overline{y_{0}}+\overline{\bar{\Omega}}\left(\bar{\sigma}-\overline{\sigma_{0}}\right)+O\left(\left(\bar{\sigma}-\overline{\sigma_{0}}\right)^{2}\right)
$$

where $\bar{\sigma}$ are the $n$ cross-section inputs. The second-order term can be ignored because of

the linearity over the range of cross-section, and the matrix $\overline{\bar{\Omega}}$, the Jacobi matrix, denotes the first derivatives of number density with respect to cross-cross section: 


$$
[\overline{\bar{\Omega}}]_{i j}=\frac{\delta y_{i}}{\delta \sigma_{j}}
$$

As stated before, the second moments of the input data are characterized by the covariance matrix, $\overline{\overline{C_{\sigma}}}$, which can be decomposed as:

$$
\overline{\overline{C_{\sigma}}}=\overline{\bar{W}} \overline{\overline{\Sigma_{\sigma}}} \overline{\bar{W}}^{T}
$$

Then the second order moments of the output data are characterized by the covariance matrix

$$
\overline{\overline{C_{y}}}=\overline{\bar{\Omega}} \overline{\overline{C_{\sigma}}} \overline{\bar{\Omega}}^{T}
$$

Combing these yields:

$$
\overline{\overline{C_{y}}}=\overline{\bar{\Omega}} \overline{\bar{W}} \overline{\overline{\Sigma_{\omega}}} \overline{\bar{W}}^{T} \overline{\bar{\Omega}}^{T}=\overline{\bar{\Omega}} \overline{\bar{W}}{\overline{\overline{\Sigma_{\omega}}}}^{1 / 2}\left(\overline{\bar{\Omega}} \overline{\bar{W}}{\overline{\overline{\Sigma_{\omega}}}}^{1 / 2}\right)^{T}
$$

The problem is that the matrix $\overline{\bar{\Omega}}$ is not available a priori, and in practice is rarely calculated. Stochastic methods build the values of $\overline{\overline{C_{y}}}$ by repeated sampling of perturbed inputs, where as ESM directly calculates $\overline{\overline{C_{y}}}$ by the following:

$$
\overline{\overline{C_{y}}}=\overline{\overline{Y_{\Sigma}}} \overline{\overline{Y_{\Sigma}}}{ }^{T} \text { where } \overline{\overline{Y_{\Sigma}}}=\left[\begin{array}{llll}
\delta \overline{y_{s 1}} & \delta \overline{y_{s 2}} & \ldots & \delta \overline{y_{s r}}
\end{array}\right]
$$

where $r$ is the rank of the input data covariance matrix and the input perturbations are $\delta \bar{\sigma}=s_{j} \overline{w_{j}}$, where $s_{j}$ is the square root of the $\mathrm{j}^{\text {th }}$ diagonal element of $\overline{\overline{\Sigma_{\sigma}}}$, and $\delta \overline{y_{s j}}$ is given by:

$$
\delta \overline{y_{s j}}=\overline{\bar{\Omega}}\left(\overline{\sigma_{0}}+s_{j} \overline{w_{j}}\right)-\overline{\bar{\Omega}}\left(\overline{\sigma_{0}}\right), j=1, \ldots, r
$$

The $\mathrm{j}^{\text {th }}$ perturbations are along the $\mathrm{j}^{\text {th }}$ singular vector of the input covariance matrix and proportional to the $\mathrm{j}^{\text {th }}$ singular value. When repeated $\mathrm{r}$ times, this procedure propagates the second moments of the input data through the model, where $r$ is the effective rank of the 
input covariance matrix $\overline{\overline{C_{\sigma}}}$, i.e. the number of singular values whose magnitudes are considered sufficiently large to not ignore. $\overline{\overline{C_{y}}}$ can now be calculated directly and, if desired, the singular value decomposition of $\overline{\overline{C_{y}}}$ can be obtained using $\overline{\overline{Y_{\Sigma}}}=\overline{\overline{U_{\Sigma}}} \overline{\overline{S_{\Sigma}}}{\overline{\overline{V_{\Sigma}}}}^{T}$ :

$$
\overline{\overline{C_{y}}}=\overline{\overline{Y_{\Sigma}}}{\overline{\overline{Y_{\Sigma}}}}^{T}=\overline{\overline{U_{\Sigma}}} \overline{\overline{S_{\Sigma}}}{\overline{\overline{V_{\Sigma}}}}^{T} \overline{\overline{V_{\Sigma}}}{\overline{\overline{S_{\Sigma}}}}^{T}{\overline{\overline{U_{\Sigma}}}}^{T}=\overline{\overline{U_{\Sigma}}}{\overline{\overline{S_{\Sigma}}}}^{2}{\overline{\overline{U_{\Sigma}}}}^{T}
$$

Implementing this within a pre-existing model is not impossible but requires a nontrivial effort and a mastery of both the linear algebra involved and computer code to perform those mathematic operations. The experiment which was used to validate this method within the simplified model and compare it to stochastic sampling created the set of $\delta \overline{y_{s j}}$ by elementary matrix operations executed in a separate program, using data especially for this one case. The model was then executed $r$ times. The data was collected into matrices by an auxiliary code and then processed by MatLab 6.5 to calculate $\overline{\overline{C_{y}}}$ as described. The numerical results validating ESM as equivalent to the stochastic approach are presented later.

When ESM was used in the more detailed TRITON model, which operates on the 44group cross sections, the methodology had to be formally implemented in a usable code. Mr. Matthew Jessee created a code that performs the above decomposition of the 44-group covariance library, $\overline{\overline{C_{\sigma}}}$, provided with SCALE 5.0, and creates perturbed 44-group crosssection libraries that can be fed directly to the TRITON model. Mr. Jessee was gracious enough to provide this resource and explain its use. This code performs the singular value decomposition of the 44-group covariance matrix, block by block, where a block is considered to be the square sub-matrix containing a single nuclide-reaction pair, and also all other nuclide-reaction pairs related to it by available covariance data. In most cases, this is 
simply the $44 \times 44$ matrix for a particular nuclide and reaction combination since the covariance data is so sparse. The largest blocks occur for uranium and the transuranics, which have covariance data because their practical significance has warranted such studies. Further, those studies are of an experimental nature in which transuranics are often so dilute in the sample that their reactions are measured as ratios to the reactions of uranium, thus producing correlation data between those reactions. A simple post-processing code was then written to handle the calculation of $\overline{\overline{C_{y}}}$.

\subsection{Computational Models Employed for Each Method}

As indicated in the introduction, this study will first use the SAS2H sequence and the ORIGEN depletion code in a simplified manner, and later the detailed TRITON sequence, all of which are available in the SCALE 5.0 package. The final model in this study uses the fast reactor code REBUS available from Argonne. For the simplified models, a particular fuel type and geometry are modeled using the SAS2H sequence supplied with a 44-group master library provided with the SCALE package which contains cross section data along with resonance parameters, Bondarenko data, flux spectrum information, scattering matrices, specific radioactivity and decay heat constants for each isotope, etc. [30]. Table 2.3 shows a brief summary of the different fuel types used in this study. More explicit definitions will appear later in the Results section, with a more detailed description, and SAS2H and ORIGEN input decks for each of the fuels included in Appendix A. 


\begin{tabular}{|l|l|l|l|}
\hline Fuel Type & Reactor & Enrichment & Geometry \\
\hline UOX & BWR & $4.5 \mathrm{w} / \mathrm{o}$ & $7 \times 7$ square lattice \\
\hline UOX & PWR & $4.5 \mathrm{w} / \mathrm{o}$ & $\begin{array}{l}17 \times 17 \text { square lattice, } 25 \\
\text { water holes }\end{array}$ \\
\hline MOX & PWR & $\begin{array}{l}1.4 \mathrm{w} / \mathrm{o} \mathrm{U}-235,8 \mathrm{w} / \mathrm{o} \mathrm{Pu}(65 \% \\
\text { fissile })\end{array}$ & $\begin{array}{l}17 \times 17 \text { square lattice, } 25 \\
\text { water holes }\end{array}$ \\
\hline MOX & PWR & $\begin{array}{l}1.4 \mathrm{w} / \mathrm{o} \mathrm{U}-235,8 \mathrm{w} / \mathrm{o} \mathrm{Pu}(65 \% \\
\text { fissile }), 1 \mathrm{w} / \mathrm{o} \mathrm{Am}, 1.5 \mathrm{w} / \mathrm{o} \mathrm{Np}\end{array}$ & $\begin{array}{l}17 \mathrm{x} 17 \text { square lattice, } 25 \\
\text { water holes }\end{array}$ \\
\hline
\end{tabular}

Table 2.3: Brief Summary of Fuel Types Examined Using SAS2H + ORIGEN

SAS2H, when given the 44-group master library, produces 44-group flux spectra.

The beginning of cycle fuel specific flux spectrum is used to collapse the 44-group covariance matrix to a 1-group covariance matrix for use in the stochastic sampling procedure described in Section 2.4. The result is a set of covariance data specific for the given fuel type being modeled. SAS2H also produces a transport updated 1-group, binary cross-section library on which the problem specific cross sections are now stored and will be used in the stand alone ORIGEN model. Note that the library for the simplified model accounts for only one representative burnup step across the life of the fuel. The following figures show the SAS2H flux spectra calculated for each of the models in Table 2.3 plotted with the typical LWR spectrum as well, where Figure 2.2 shows the PWR fuels for both UOX and MOX, and Figure 2.3 shows the BWR fuel at various void fractions. Unlike the later TRITON model, fluxes from SAS2H are not normalized to the same fuel specific power density, but the input power applied to all materials in the model. 


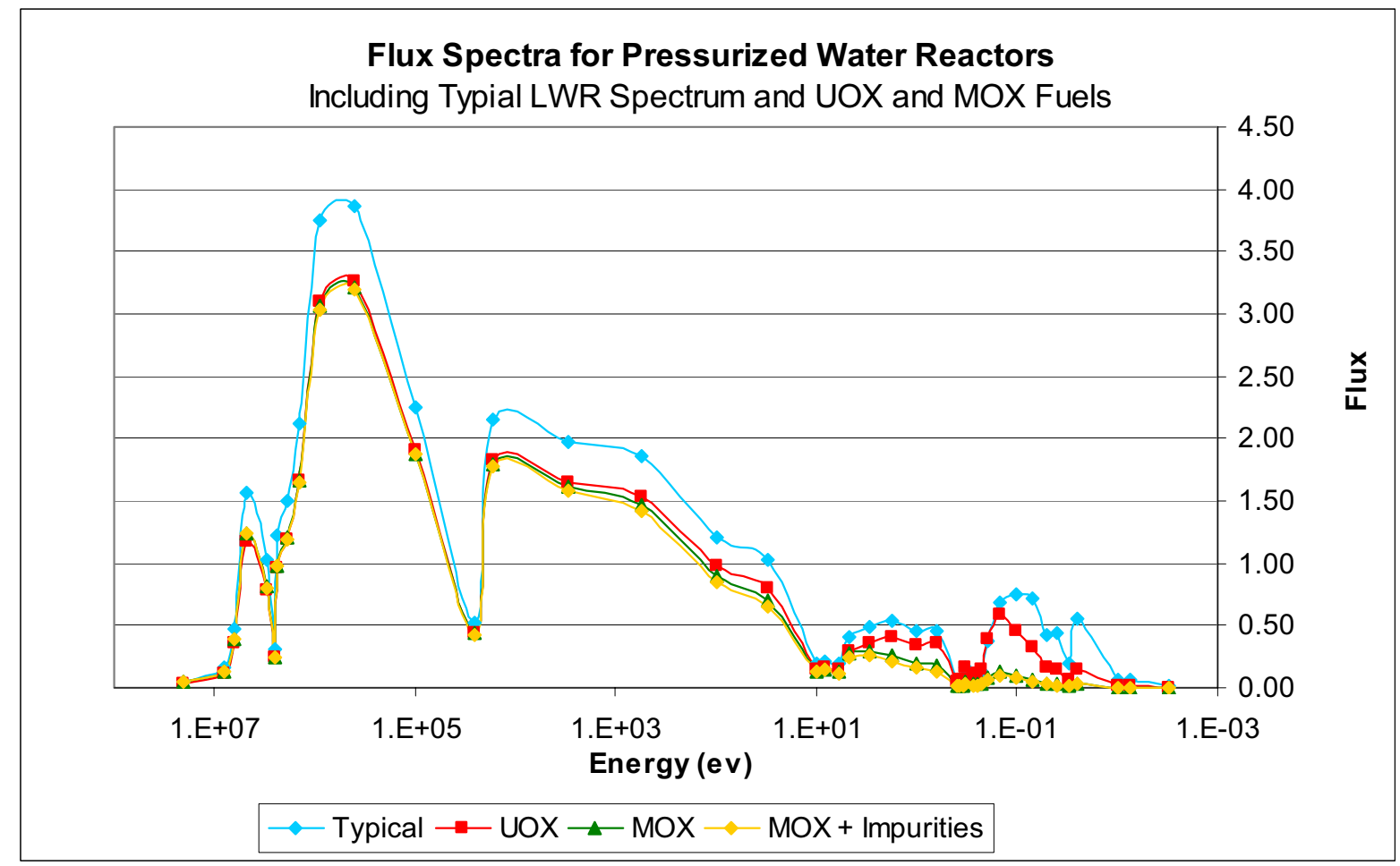

Figure 2.2: Flux Spectra for PWR Models

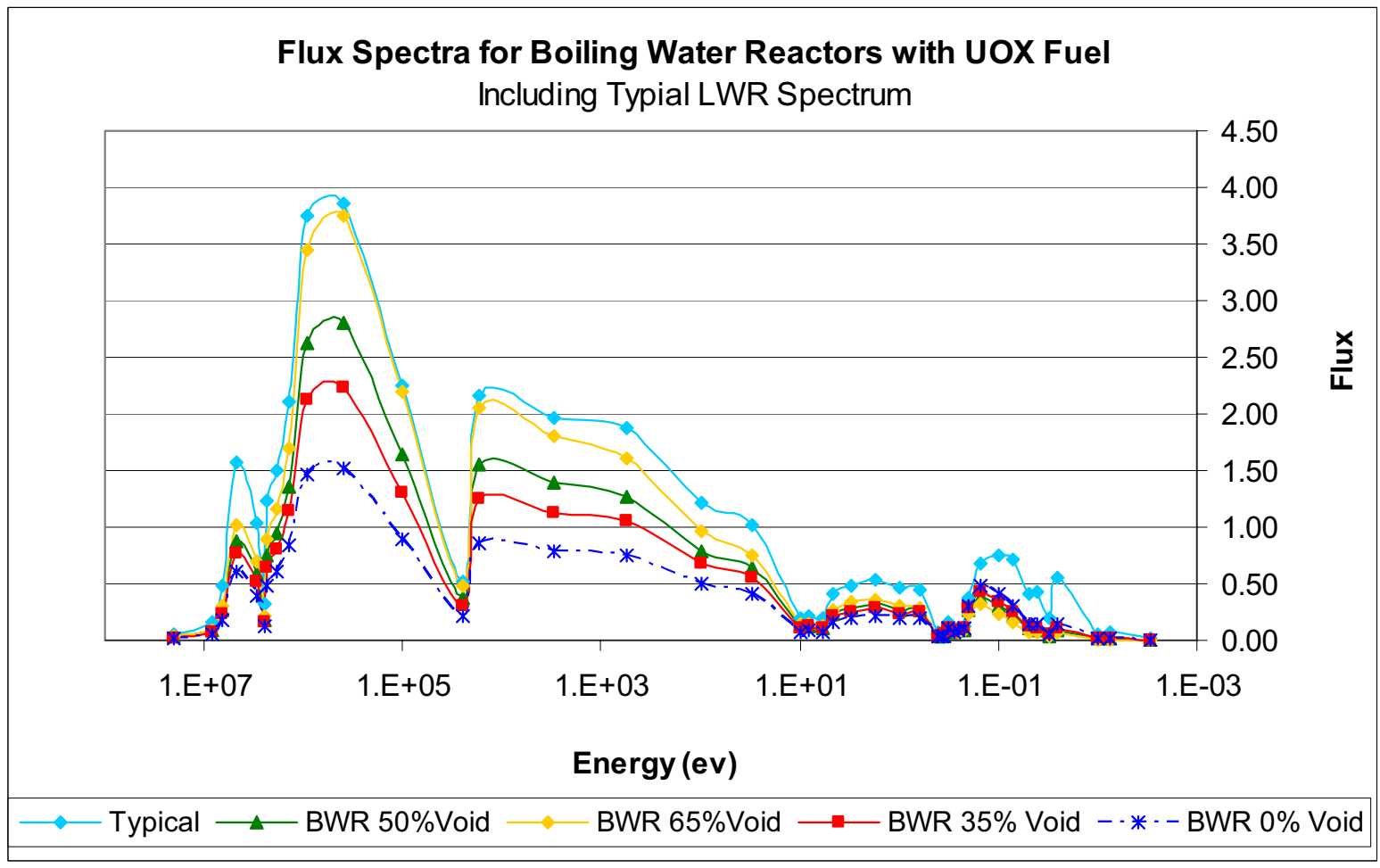

Figure 2.3: Flux Spectra for BWR Models 
The stand-alone ORIGEN model is defined as 1 metric ton of the fuel modeled by SAS2H. ORIGEN depletes the model using the same power history and burnup as specified in the SAS2H model, then proceeds to decay the discharged isotopics over a series of time steps between discharge and 10,000 years, which covers the reprocessing time-frame through when waste canister failures are assumed in a waste repository. ORIGEN uses the fuel specific cross sections that were created by SAS2H for a specific fuel with a set of specific power cycle parameters. The nominal ORIGEN case, which is run with the unperturbed cross sections, creates the nominal values for the model. The nominal ORIGEN model is set to output not only the discharge isotopic masses, but also the isotopic and total values for each of the metrics of interest. Specific values for decay heat, activity, and toxicity can be obtained by dividing the metric by the mass or by directly printing the specific values from the binary library. The ORIGEN model, into which perturbations are introduced, is set to determine only the masses, since they are all that will be needed for statistical evaluation, as will be described in the next section. One-group perturbations are created a priori for the stochastic sampling method for each fuel and for the ESM comparison experiment. The perturbations, as described in the previous sections, are then introduced directly into the cross sections as they are read into ORIGEN from the binary library by interrupting the code at that point and perturbing the cross-sections that covariance data are available for. A code was written to run ORIGEN for $\mathrm{N}$ samples and then acquire the number densities of the tracked nuclides from each sample (see Table 2.4) and group the results by the various decay times. The nuclides tracked are chosen mainly for their contribution to decay heat or toxicity, some of which are only chemically toxic rather than a producer of non-negligible radiation. Moreover, together the tracked nuclides represent greater than $95 \%$ of heat, radioactivity, and 
radiotoxicity for any time greater than 10 years for all of the fuels modeled in this work. A nominal execution of ORIGEN takes approximately eight seconds to execute on desktop PC and the sampling version that introduces perturbations takes approximately seconds to execute on the same platform.

\begin{tabular}{|l|l|l|l|l|l|l|}
\hline Pb-210 & Pa-231 & U-238 & Pu-240 & Am-243 & Se-79 & Cs-134 \\
\hline Ra-226 & U-234 & Np-237 & Pu-241 & Cm-242 & Sr-90 & Cs-137 \\
\hline Ac-227 & U-235 & Np-239 & Pu-242 & Cm-244 & Y-90 & Ba-137m \\
\hline Th-227 & U-236 & Pu-238 & Am-241 & Cm-245 & Tc-99 & \\
\hline Th-230 & U-237 & Pu-239 & Am-242m & C-14 & I-129 & \\
\hline
\end{tabular}

Table 2.4: Isotopes Tracked for Analysis

The TRITON model was used only for the fast reactors fuels and a validation for the pressurized water reactor fuel. For the purposes of this research, TRITON will be run as a stand-alone model using the 44-group master library as an input. The choice was made to fully exploit TRITON's detailed modeling abilities as this was the natural progression from the cruder light water reactor models already described. For each of the fast reactor fuels modeled, and a 4.5 w/o uranium oxide fuel, detailed geometries of the unit cell and fuel composition defined by volume fractions were entered into the input. The biggest difference, besides going from simple ORIGEN depletion to a rigorous 2-D transport, was burning the fuel over $25+$ smaller burnup steps with both cross-section and flux spectrum updates for each step as opposed to a single, representative step employed in the ORIGEN model with only one cross section update at mid-point of that step. At the end of the TRIRON execution, the discharge number densities of the same isotopes are decayed using the same time steps as in previous models. 


\begin{tabular}{|l|l|l|l|}
\hline Fuel Type & Reactor & Enrichment & Geometry \\
\hline UOX & PWR & $4.5 \mathrm{w} / \mathrm{o}$ & $\begin{array}{l}17 \mathrm{x} 17 \text { square lattice, } 25 \text { water } \\
\text { holes }\end{array}$ \\
\hline $\begin{array}{l}\text { Actinide, } \\
\text { metal }\end{array}$ & $\begin{array}{l}\mathrm{FR}, \\
\mathrm{CR}=0.25\end{array}$ & $\begin{array}{l}59.2 \mathrm{w} / \mathrm{o} \text { transuranics, } 20 \mathrm{w} / \mathrm{o} \\
\text { zirconium }\end{array}$ & 217 pin hexagonal lattice \\
\hline $\begin{array}{l}\text { Actinide, } \\
\text { metal }\end{array}$ & $\mathrm{FR}$, & $\begin{array}{l}20.6 \mathrm{w} / \mathrm{o} \text { transuranics, } 10 \mathrm{w} / \mathrm{o} \\
\text { zirconium }\end{array}$ & 169 pin hexagonal lattice \\
\hline $\begin{array}{l}\text { Actinide, } \\
\text { metal }\end{array}$ & $\begin{array}{l}\mathrm{FR}, \\
\mathrm{CR}=1.05\end{array}$ & $\begin{array}{l}16.2 \mathrm{w} / \mathrm{o} \text { transuranics, } 10 \mathrm{w} / \mathrm{o} \\
\text { zirconium }\end{array}$ & 127 pin hexagonal lattice \\
\hline
\end{tabular}

Table 2.5: Brief Summary of Fuel Types Examined Using TRITON

Since TRITON takes approximately twenty to thirty seconds per burnup step to execute and has, in principle, 44 times as much input data, it is apparent that stochastic sampling is not a reasonable method to use with this model. Not only does TRITON take longer to execute, but it also uses the 44-group master cross section library as input and thus the 44-group covariance library as a source for perturbations. The principles of Monte Carlo sampling indicate that many thousands of samples would have to be run to properly propagate the uncertainties. In going from 1-group to 44-group covariance data the effective rank of the covariance matrix increased from 223 to 1938, with cutoff criteria imposed on singular values with a magnitude less than $10^{-6}$ relative to the reference cross section. So even ESM will require the execution of the code 1938 times, but this is still far less than computationally taxing than running over 3000 samples which is on the order of the input data. For each sample set, perturbations according to the ESM method are introduced directly into the 44-group master library, using a code generously provided by Mr. Jessee, and a new perturbed library is created which is subsequently input to the TRITON model. It was concluded that the primary depletion model is nearly linear from the linearity study of ORIGEN, but in order to confidently avoid non-linearities that may arise if cross sections are perturbed outside the linear region, instead of multiplying by the square root of the 
eigenvalue, the perturbation is scaled by a scaling factor, $\mathrm{SCF}=0.07$, divided by the infinity norm of the eigenvector being used in a particular sample.

$$
\bar{\sigma}=\overline{\sigma_{0}}\left(I+\frac{S C F}{\left\|\overline{w_{i}}\right\|_{\infty}} \overline{w_{i}}\right)
$$

The scaling factor and infinity norm are divided out in post processing, and the singular value is multiplied back into the output when computing the covariance matrix of the output.

$$
\begin{gathered}
\delta y_{i}=\left[\Omega\left(\overline{\sigma_{0}}+\frac{S C F}{\left\|\overline{w_{i}}\right\|_{\infty}} \overline{w_{i}}\right)-\Omega\left(\overline{\sigma_{0}}\right)\right] \frac{\left\|\overline{w_{i}}\right\|_{\infty}}{S C F} \\
\overline{\overline{C_{y}}}=\left(\overline{\overline{Y_{\Sigma}}} \overline{\bar{S}}^{1 / 2}\right)\left(\overline{\overline{Y_{\Sigma}}}=1 / 2\right)^{T}
\end{gathered}
$$

The covariance matrix for output isotopics directly provides the uncertainty information needed for this study, namely the standard deviation of each isotope tracked.

The ESM sampling method was similarly implemented in REBUS by Dr. AbdelKhalik. Both TRITON and REBUS will be used for modeling recycling of the fast reactor fuel corresponding to the conversion ratio of about 0.70 . As discussed before, given material feeds and reprocessing parameters, REBUS does this automatically. A external procedure is developed and implemented for TRITON to emulate this recycling methodology. That procedure is described in the following section. The recycle procedure of taking all the fast reactor transuranics and combining them with spent LWR fuel is that which is outlined in Argonne's ABTR Preconceptual Design Report [26]. 


\subsection{TRITON Recycle Methodology}

Having evaluated the resulting isotopic covariance matrices for once-through fuels in both thermal and fast reactors, our attention turns to a recycling scheme. The recycling scheme creates a transuranic fuel, made of spent LWR fuel, burns it in a fast reactor, and then recycles that fuel back into the fast reactor, making up part of the fuel mass by adding more spent LWR fuel to the mix. The transuranics recycled are Np-237, Np-239, Pu-238, Pu-239, Pu-240, Pu-241, Pu-242, Am-241, Am-242m, Am-243, Cm-242, Cm-244, and Cm-245. The first step is to create a nominal recycle case by taking mass $M_{F R}^{\text {Recycle }}$ from 1.5 year decayed fast reactor fuel and adding mass $\mathrm{M}_{\mathrm{LWR}}$ from $10 \mathrm{yr}$ decayed thermal reactor fuel (burnup of $33 \mathrm{GWD} / \mathrm{MTU}$ and an original enrichment of $3.3 \mathrm{w} / \mathrm{o}$ ) and mass $\mathrm{M}_{\mathrm{DU}}$ from a depleted uranium source to create a new fuel having the same volume loading as the original fuel, and thus approximately the same total heavy metal mass, given by:

$$
M_{F R}^{B O L}=M_{F R}^{\text {Re cycle }}+M_{L W R}^{\mathrm{Re} c y c l e}+M_{D U}
$$

Here, an assumption about the type of reprocessing is made, e.g. UREX, perfect separation, etc. In this work, all the transuranics from the spent fast reactor fuel are added back and enrichment made up by LWR spent fuel transuranics, $M_{L W R}^{\text {Recycle }}$, with the remaining mass being depleted uranium, $M_{D U}$. To obtain the additional equation required to solve for $M_{L W R}^{\text {Recyle }}$ and $M_{D U}$, it is required that the composition of $M_{F R}^{B O L}$ be such so as to achieve the

cycle energy requirement. $M_{F R}^{\text {Recycle }}$ is therefore all the mass of the recycled fuel extracted after $M_{F R}^{B O L}$ is burnt and reprocessed. This implies the following relationship: 


$$
M_{F R}^{B O L}-M_{F R}^{\mathrm{Re} c y c l e}=M_{L W R}^{\mathrm{Re} c y c l e}+M_{D U}
$$

The depleted uranium is assumed to have a fixed isotopic composition of $99.8 \mathrm{w} / \mathrm{o} \mathrm{U}-238$ and 0.2 w/o U-235. Further, the isotopic compositions of the LWR and FR fuels are known as well, so that when the masses are combined, masses of individual isotopes add. Since the TRITON model requires input isotopics to be in $\mathrm{w} / \mathrm{o}, \mathrm{M}_{\mathrm{R}}$ must be expanded in terms of its composition, completed as now explained. Treat the mass as a vector composed of isotopes from element $k: \overline{M_{F R, k}^{B O L}}$. Convert the masses in $\overline{M_{F R, k}^{B O L}}$ to weight percents by $\frac{\overline{M_{F R, k}^{B O L}}}{\| \overline{M_{F R, k}^{B O L} \|_{1}}}$, where the one-norm is the sum of the masses of all isotopes of element $k$. The last expression gives the isotopic data for element $k$ that will be input to the TRITON model. This process is repeated for each element $k$. For this experiment, the discharged isotopics and end of life keffective values from the initial fresh fuel TRITON input are taken to be the target values, despite k-effective being greater than 1 . The output data from the unperturbed TRITON model are considered to be the nominal values for each recycle step. The k-effective values are not only recorded for statistical analysis, but are also used to adjust the transuranic enrichment for the next recycle, in an effort to maintain the end of life k-effective value. Next, let $\overline{\overline{C_{L W R}^{\text {Recycle }}}}$ and $\overline{\overline{C_{F R}^{\text {Recycle }}}}$ be the absolute isotopic covariance matrices of the once-through thermal and fast reactors, respectively, and converted, if necessary, from their relative values to units of mass. The fast reactor data are taken 1.5 years after discharge and the thermal reactor data are taken at 10 years of decay, i.e. time lapse to recycle. These matrices are 13 x 13 containing number density uncertainties for the transuranics already mentioned. These two matrices are the results of earlier work with each of these fuel types 
using the TRITON model with our ESM approach to propagate uncertainties due to crosssections. We now wish to recycle the fuel characterized by these uncertainties.

The masses of each isotope of each element in each fuel stream is perturbed

separately using $\overline{\overline{C_{L W R}^{\text {Recycle }}}}$ and $\overline{\overline{C_{F R}^{\text {Recycle }}}}$, via the ESM method. Each matrix is decomposed such that:

$$
\overline{\overline{C^{\text {Recycle }}}}=\overline{\overline{W^{\text {Recycle }}}} \overline{\overline{\sum^{\text {Recycle }}}}{\overline{\bar{W}^{\text {Recycle }}}}^{T}
$$

Perturbations are introduced into the masses of the 13 isotopes in that fuel stream by scaling a singular vector by the square root of the corresponding singular value and adding this perturbation vector to the mass vector. These perturbed isotopics are then used to satisfy equation 2.29, just as the unperturbed values would be. Finally, just as in the unperturbed case, the masses of each element are converted to weight percents as required by TRITON's input structure, and a perturbed input written for the model.

Finally, uncertainty is propagated by running the model to equilibrium several times, each time choosing a subsequent singular pair to perturb with, i.e. perturbing along $w_{1}, w_{2}$, etc. with each new run of the model. Experimentation revealed that only the first six singular pair perturbations needed to be run to effectively propagate the uncertainty, which is in accordance with the theory of ESM. The above procedure is repeated each time the fuel is recycled, thus creating a new $\overline{\overline{C_{F R}^{B O L}}}$ and a new decomposition to perturb by, for each recycle step.

The resulting recycled fuel nuclei number density uncertainties are combined with the nuclei number density uncertainties due to cross-section uncertainties into a single uncertainty vector, $\overline{\sigma_{\text {TOTAL }}}$, where the elements of the vector denote different isotopes. 
Experience showed that nuclei number density uncertainties due to cross-section uncertainties changed little unless major compositional changes are made to the fuel (see Results section). In the following, the subscript $F R$ denotes recycled fuel nuclei number density uncertainties at EOL originating from the uncertainty of the material making up the fuel independent of cross section uncertainties, and the subscript $X S$ denotes fuel nuclei number density uncertainties at EOL originating from cross-section uncertainties:

$$
\left(\overline{\sigma_{\text {TOTAL }}}\right)_{i}=\sqrt{\left(\overline{\sigma_{X S}}\right)_{i}^{2}+\left(\overline{\sigma_{F R}}\right)_{i}^{2}+2\left(\overline{\sigma_{X S}}\right)_{i}\left(\overline{\sigma_{F R}}\right)_{i}\left(\rho_{i}\right)}
$$

where $\rho$ is a correlation between the two uncertainties. Having the total uncertainty on the recycled fuel composition at equilibrium, the mass uncertainties, and in turn the key metrics uncertainties, for any given discharged fuel that will be sent to permanent disposal can be computed. Values of operational parameters (e.g. k-effective) can be collected from the model both at equilibrium and between recycle steps, and the uncertainties on those parameters computed as well.

Since the cross section originated uncertainties on EOL isotopics affect the subsequent reload isotopics, there must be correlation. However, within the scope of this work, it is assumed that $\rho=0$, because the only foreseeable method to obtain that correlation with the TRITON model is a posteriori calculation from the results of running the possible cross-section uncertainties with each of the possible recycle nuclei number density uncertainties, through equilibrium. This task is currently too computationally taxing as it would require execution time of $(15$ minutes per execution $)\left(r_{X S}+1\right)\left(r_{R}+1\right)(6+1)$ times, where 1 is for the nominal case and $r_{X S}$ and $r_{R}$ are the effective ranks of the covariance matrices for cross-sections and recycled isotopics, respectively. 


\subsection{Statistical Analysis Performed on Results}

The forward perturbation sampling process produces a large group of data for each fuel type; these raw data are in a form in which it can be processed using rudimentary statistical methods. The ESM sampling method directly calculates the covariance matrix of the isotopics in post processing, as already discussed, so much of this section applies only to the simplified ORIGEN models rather than the TRITON and REBUS models. First, the unperturbed values of the fuel sample produced by ORIGEN -- isotopics and total heat, radioactivity and radiotoxicity -- using the problem specific cross-sections provided by the SAS2H model are, by definition, the mean and most likely values for the particular model. For the thirty-three nuclides tracked, there are $\mathrm{N}$ samples of isotopic masses at discharge and $1,5,10,50,100,500,1000,2500,5000$, and 10000 years of decay. The values at discharge and 1 year are neglected in the statistical analysis as many very-short lived isotopes present at this time contribute much of the heat load in the first 1 year or so until they die off. Thus, the thirty three nuclides tracked would not cover greater than $95 \%$ of the heat, etc. that is of interest. This is practically justified by the fact that the fuel will be closely monitored and guarded in wet storage for at least the first five years, and this work is mainly concerned with the affects on the repository and reprocessing aspects which take place later.

Basic statistics are applicable to this data because all the metrics are linearly and directly proportional to mass, therefore each have specific values. Each isotope tracked has some specific constant for heat $[\mathrm{W} / \mathrm{g}]$, activity $[\mathrm{Ci} / \mathrm{g}]$, and radiotoxicity $\left[\left(\mathrm{m}^{3}\right.\right.$ air or water to dilute to acceptable leve)/g]. The statistical process is justified by the equation: 


$$
\mu_{y}=\left(\frac{d y}{d x}\right) \mu_{x}
$$

which states that the uncertainty in a parameter $\mathrm{y}$ that is dependent upon parameter $\mathrm{x}$ is simply the derivative of the relationship that relates the two times the uncertainty in $\mathrm{x}$ [28]. For all the metrics of interest, a change in the metric is simply the change in mass times the specific value for that metric. All that is needed, therefore, are the statistics of the isotopic masses which can be translated to the metrics by means of these specific values, which are given in Table 2.6. 


\begin{tabular}{|c|c|c|c|c|}
\hline Nuclide & Ci/Gram & $\begin{array}{l}\text { Heat/Gram } \\
\text { (W) }\end{array}$ & $\mathrm{m}^{3}$ Air/g & $\mathrm{m}^{3}$ Water/g \\
\hline pb210 & $7.6376 \mathrm{E}+01$ & $1.7901 \mathrm{E}-02$ & NA & NA \\
\hline ra226 & $9.8912 \mathrm{E}-01$ & $2.8565 \mathrm{E}-02$ & NA & NA \\
\hline ac227 & $7.2373 E+01$ & $3.5003 \mathrm{E}-02$ & $2.3886 \mathrm{E}+16$ & NA \\
\hline th227 & $3.0749 E+04$ & $1.1235 \mathrm{E}+03$ & $1.8412 \mathrm{E}+17$ & NA \\
\hline th230 & 2.0627E-02 & $5.8238 \mathrm{E}-04$ & $1.2352 \mathrm{E}+12$ & NA \\
\hline pa231 & 4.7253E-02 & 1.4378E-03 & $3.9708 \mathrm{E}+12$ & NA \\
\hline u234 & $6.2204 \mathrm{E}-03$ & 1.7905E-04 & $3.5144 \mathrm{E}+10$ & $1.6456 \mathrm{E}+04$ \\
\hline $\mathrm{u} 235$ & 2.1624E-06 & $5.9912 \mathrm{E}-08$ & 1.1033E+07 & $5.4884 \mathrm{E}+00$ \\
\hline $\mathrm{u} 236$ & 6.4706E-05 & 1.7521E-06 & $3.3701 \mathrm{E}+08$ & $1.6423 \mathrm{E}+02$ \\
\hline $\mathrm{u} 237$ & $8.1658 \mathrm{E}+04$ & $1.5809 \mathrm{E}+02$ & $9.3110 \mathrm{E}+13$ & $3.3604 \mathrm{E}+09$ \\
\hline $\mathrm{u} 238$ & 3.3633E-07 & $8.5129 \mathrm{E}-09$ & $1.6170 \mathrm{E}+06$ & 8.1832E-01 \\
\hline np237 & $7.0521 \mathrm{E}-04$ & 2.0119E-05 & $2.1177 \mathrm{E}+10$ & $4.1977 \mathrm{E}+03$ \\
\hline np239 & $2.3206 \mathrm{E}+05$ & $5.8682 \mathrm{E}+02$ & $1.3896 \mathrm{E}+14$ & $1.0046 \mathrm{E}+10$ \\
\hline pu238 & $1.7132 \mathrm{E}+01$ & 5.6779E-01 & $1.1271 \mathrm{E}+15$ & $2.1309 \mathrm{E}+08$ \\
\hline pu239 & $6.2072 \mathrm{E}-02$ & $1.9291 \mathrm{E}-03$ & $4.4656 \mathrm{E}+12$ & $8.3881 \mathrm{E}+05$ \\
\hline pu240 & $2.2708 \mathrm{E}-01$ & 7.0707E-03 & $1.6336 \mathrm{E}+13$ & $3.0686 \mathrm{E}+06$ \\
\hline pu241 & $1.0343 \mathrm{E}+02$ & $3.2868 \mathrm{E}-03$ & $1.4266 \mathrm{E}+14$ & $2.6864 \mathrm{E}+07$ \\
\hline pu242 & $3.9558 \mathrm{E}-03$ & 1.1682E-04 & $2.6025 \mathrm{E}+11$ & $5.1307 \mathrm{E}+04$ \\
\hline am241 & $3.4309 \mathrm{E}+00$ & 1.1448E-01 & $1.9718 \mathrm{E}+14$ & $3.7091 \mathrm{E}+07$ \\
\hline am242m & $1.0481 \mathrm{E}+01$ & 4.2370E-03 & $5.7904 \mathrm{E}+14$ & $1.0760 \mathrm{E}+08$ \\
\hline am243 & 1.9969E-01 & $6.4285 \mathrm{E}-03$ & $1.1476 \mathrm{E}+13$ & $2.1588 \mathrm{E}+06$ \\
\hline $\mathrm{cm} 242$ & $3.3124 E+03$ & $1.2085 E+02$ & $1.1705 \mathrm{E}+16$ & $2.1509 \mathrm{E}+09$ \\
\hline $\mathrm{cm} 244$ & $8.0981 E+01$ & $2.8322 E+00$ & $2.7733 \mathrm{E}+15$ & $5.2585 \mathrm{E}+08$ \\
\hline $\mathrm{cm} 245$ & 1.7177E-01 & $5.7170 \mathrm{E}-03$ & $1.0224 \mathrm{E}+13$ & $1.9497 \mathrm{E}+06$ \\
\hline c 14 & $4.4584 \mathrm{E}+00$ & 1.3074E-03 & $1.5535 \mathrm{E}+10$ & NA \\
\hline se 79 & 1.5362E-02 & $5.0813 \mathrm{E}-06$ & $6.2704 \mathrm{E}+07$ & $2.4079 \mathrm{E}+03$ \\
\hline sr 90 & $1.4117 \mathrm{E}+02$ & 1.6393E-01 & $1.3574 \mathrm{E}+13$ & $2.1357 \mathrm{E}+08$ \\
\hline tc 99 & 1.7114E-02 & $8.5821 \mathrm{E}-06$ & $1.3370 \mathrm{E}+08$ & $5.9217 \mathrm{E}+02$ \\
\hline $\mathrm{i} 129$ & 1.7659E-04 & $8.2592 \mathrm{E}-08$ & $1.0149 \mathrm{E}+07$ & $1.0512 \mathrm{E}+03$ \\
\hline cs137 & $8.7021 \mathrm{E}+01$ & $9.6718 \mathrm{E}-02$ & $2.0380 \mathrm{E}+12$ & $6.1282 \mathrm{E}+07$ \\
\hline ba137m & $5.3801 \mathrm{E}+08$ & $2.1138 \mathrm{E}+06$ & NA & $5.3801 \mathrm{E}+08$ \\
\hline y90 & $5.4342 \mathrm{E}+05$ & $3.0086 \mathrm{E}+03$ & $4.8957 \mathrm{E}+14$ & $7.9331 \mathrm{E}+10$ \\
\hline cs134 & $1.2944 E+03$ & $1.3197 \mathrm{E}+01$ & $1.5521 \mathrm{E}+13$ & $1.3290 \mathrm{E}+09$ \\
\hline
\end{tabular}

Table 2.6: Specific values, per nuclide, for metrics of interest.

For each isotope tracked, the sample mean, $\mu_{\mathrm{m}}$ and sample standard deviation, $\Sigma_{\mathrm{SD}}$ are calculated using the equations below [28]. 


$$
\begin{gathered}
\mu_{m}=\frac{1}{N} \sum_{i=1}^{N} x_{i} \\
\Sigma_{S D}=\sqrt{\frac{1}{N-1} \sum_{i=1}^{N}\left(x_{i}-\mu_{m}\right)^{2}}
\end{gathered}
$$

The standard deviation of the isotopic masses is the key metric needed for the analysis. Thus it must be justified that the standard deviation calculated is from a reasonably accurate sample. Comparison is made between the sampled mean and the true mean in terms of the expected standard deviation of the mean, $\Sigma_{\mathrm{m}}$, calculated by:

$$
\Sigma_{m}=\frac{\Sigma_{S D}}{\sqrt{N}}
$$

This is a modification of the Central Limit Theorem, where the value of the mean of the sample is expected to deviate from the true value for a finite number of samples [28]. If the difference between the sampled mean and the true mean is within two or fewer standard deviations of the mean, the sample can be said to be reasonable. Finally, the convergence of the mean and the standard deviation are examined, i.e. after how many samples do they reach a nearly constant value. Those values tended toward 200 for the mean and 220-250 for the standard deviation so $\mathrm{N}=300$ samples is adequate. The standard deviation of the mass translates directly into uncertainties for each of the metrics observed, i.e. $+/-5 \%$ in mass produces $+/-5 \%$ in heat, etc. Also, consider the objective of a $95 \%$ confidence interval, using the student- $t$ distribution, the standard deviation is multiplied by 1.96 to obtain the $95 \%$ confidence interval rather than just one standard deviation which provides approximately $68 \%$ confidence [28].

Next, the affect of the uncertainty on the total values is examined. The resulting uncertainties, $\mu_{\mathrm{R}}$, are propagated by the square root of the sum of the squares [28]: 


$$
\mu_{R}=\sqrt{\sum_{i=1}^{L} \mu_{m, i}^{2}}
$$

where $\mathrm{L}$ is the number of parameters, i.e. tracked nuclides. This equation, by definition, assumes that there is no correlation between each isotopic metric, e.g. the heat produced by plutonium does not affect the heat produced by strontium. This produces a total uncertainty in that metric as contributed by the nuclides tracked, which can be compared to the nominal value of that metric for the particular fuel type and decay time. This is done not only to see how much uncertainty is imparted to the metric by the uncertainties of these isotopes, but also to verify that in tracking the specific 33 nuclides at least $95 \%$ of the total heat, etc. for that discrete point in time is observed.

Finally, for the fast reactor fuels modeled in TRITON and REBUS, the isotopics covariance matrix was constructed using the algorithm discussed in Section 2.5. The square roots of the diagonal elements represent the standard deviations of each of the tracked nuclides, equivalent to the standard deviations calculated from stochastic sampling. Also, in the TRITON results, the sub-matrix containing the actinides Np-237 through Cm-244 is extracted to be used for uncertainty propagation when examining recycling of fast reactor fuels. 


\section{Numerical Results}

\subsection{Simplified ORIGEN Models}

\subsubsection{Equivalency of ESM and Stochastic Methods}

The essential benefit of ESM is that it will produce the same results as stochastic sampling and will work efficiently in models where stochastic methods would be impractical to implement. To verify this numerically, both ESM and the full stochastic sampling are implemented for the simplified PWR model, which is simple enough to allow either method. The 1-group covariance matrix is decomposed and 223 perturbations $(r a n k=223)$ are created according to the formulas already discussed. Perturbations are introduced directly within ORIGEN, using the cross section library made by SAS2H for the PWR fuel. Considering the time required to implement this method versus the fast execution time of the simple stochastic model, ESM is not well suited to small, simple models. In more sophisticated models where the runtime increases greatly and stochastic methods are not practical, however, ESM becomes worth the time it takes to implement.

Table 3.1 presents the comparison of the isotopics' uncertainties predicted by each method and Table 3.2 gives the nominal discharge isotopics for this model for reference. It is clear from these results that the two methods are producing equivalent results, as expected. A further look at the simplified PWR model is included in the next section. Note here, and in subsequent sections, only the discharge isotopics and the isotopic uncertainties for each model will be presented, as number density vs. time is calculated from the decay of the discharge isotopics. A larger, generalized results table for decay heat, radioactivity, 
radiotoxicity, and uncertainty contributors is available for each model, but due to their size, have been included in Appendix $\mathrm{C}$ and the reader is referred to that section. The tables included there appear in the order in which models are presented in the main text.

\begin{tabular}{|l|r|r|}
\hline \multirow{2}{*}{ Nuclide } & \multicolumn{2}{|c|}{ \% Uncertianty } \\
\cline { 2 - 3 } & Stochastic & ESM \\
\hline pb210 & 1.1951 & 1.1477 \\
\hline ra226 & 1.3548 & 1.3008 \\
\hline ac227 & 0.4805 & 0.4575 \\
\hline th227 & 0.4805 & 0.4578 \\
\hline th230 & 1.4910 & 1.4327 \\
\hline pa231 & 0.5177 & 0.4957 \\
\hline u234 & 1.5787 & 1.5193 \\
\hline u235 & 1.3125 & 1.3377 \\
\hline u236 & 0.7124 & 0.7017 \\
\hline u237 & 2.5207 & 2.5509 \\
\hline u238 & 0.0775 & 0.0759 \\
\hline np237 & 0.6054 & 0.6158 \\
\hline np239 & 13.6428 & 13.8702 \\
\hline pu238 & 1.0497 & 1.0579 \\
\hline pu239 & 0.8064 & 0.8851 \\
\hline pu240 & 2.5656 & 2.7661 \\
\hline pu241 & 2.5207 & 2.5509 \\
\hline pu242 & 2.5983 & 2.6248 \\
\hline am241 & 2.5016 & 2.5345 \\
\hline am242m & 2.1720 & 2.2293 \\
\hline am243 & 13.6428 & 13.8698 \\
\hline cm242 & 2.1717 & 2.2289 \\
\hline cm244 & 11.3402 & 11.6702 \\
\hline cm245 & 10.2508 & 10.5995 \\
\hline c 14 & 0.4523 & 0.4900 \\
\hline se 79 & 0.3663 & 0.3825 \\
\hline sr 90 & 0.3748 & 0.3853 \\
\hline tc 99 & 1.9704 & 1.8803 \\
\hline i129 & 0.4186 & 0.4487 \\
\hline cs137 & 0.3822 & 0.3986 \\
\hline ba137m & 0.3822 & 0.3988 \\
\hline y90 & 0.3748 & 0.3857 \\
\hline cs134 & 1.1380 & 1.2094 \\
\hline sm
\end{tabular}

Table 3.1: Comparison of isotopic uncertainties from the two methods. 


\begin{tabular}{|c|c|c|c|c|c|}
\hline \multicolumn{6}{|c|}{ Discharge Isotopics, grams / MTHM } \\
\hline pb210 & $2.415 \mathrm{E}-11$ & np237 & $5.538 \mathrm{E}+02$ & cm244 & $3.215 \mathrm{E}+01$ \\
\hline ra226 & $1.628 \mathrm{E}-08$ & np239 & $8.925 \mathrm{E}+01$ & cm245 & $1.208 \mathrm{E}+00$ \\
\hline ac227 & 3.443E-09 & pu238 & $1.791 \mathrm{E}+02$ & c 14 & 3.335E-03 \\
\hline th227 & 1.014E-11 & pu239 & & se 79 & $5.817 \mathrm{E}+00$ \\
\hline th230 & $1.140 \mathrm{E}$ & pu240 & 1.88 & sr 90 & $6.899 \mathrm{E}+02$ \\
\hline pa231 & 3.116E-04 & pu241 & $\mathrm{E}+03$ & tc 99 & $9.577 \mathrm{E}+02$ \\
\hline u234 & 1.530 & & $6 \mathrm{E}+02$ & i129 & $1.741 \mathrm{E}+02$ \\
\hline u235 & $1.233 \mathrm{E}+04$ & am241 & $4.302 \mathrm{E}+01$ & cs137 & $1.485 \mathrm{E}+03$ \\
\hline u236 & $5.524 \mathrm{E}+03$ & am242m & 9.197E-01 & ba137m & $2.283 \mathrm{E}-04$ \\
\hline u237 & $1.346 \mathrm{E}+01$ & am243 & $1.162 \mathrm{E}+02$ & y90 & $1.866 \mathrm{E}-01$ \\
\hline u238 & $9.298 \mathrm{E}+05$ & cm242 & $1.346 \mathrm{E}+01$ & cs134 & $1.439 \mathrm{E}+02$ \\
\hline
\end{tabular}

Table 3.2: Discharge isotopics for the PWR simplified model.

\subsubsection{PWR Model with UOX Fuel}

The following data are for a representative UOX fuel that is burned in a pressurized water reactor. An updated cross-section library is created using SAS2H for one representative burnup step, and the resulting 1-group working library used with ORIGEN. The 44-group covariance library is collapsed to 1-group using the beginning of cycle flux spectrum for this fuel, and stochastic sampling is implemented. The UOX fuel is $4.5 \mathrm{w} / \mathrm{o}$ and burned to $40 \mathrm{GWD} / \mathrm{MTU}$ in a single cycle representative of a once-through fuel. The geometry is a 17x17 Westinghouse fuel assembly with no burnable poison elements and 25 water holes with one being an instrumentation hole; adapted from Gauld [21]. See Appendix A for a more detailed description of this model. Since the discharge isotopics and the isotopic uncertainties for this model have already been presented in the previous section, and the results table is available in Appendix C, the discussion moves on to the separation study of this fuel.

This simple experiment examines the affect of decay heat uncertainties in the process of UOX fuel separation, a key aspect of fuel reprocessing. The simple model 4.5 w/o UOX that was burned to $40 \mathrm{GWD} / \mathrm{MTU}$ is decayed in three separate cases for 5, 10 and 25 years 
(Table 3.3). At each individual time the uranium, plutonium, neptunium, americium, and curium were separated out by elemental species (henceforth referred to as lumps) with assumed 100\% separation efficiency. The lumps were then decayed over the 10,000 year time, regardless of the lump's separation time or decay products. The heat load of each of these lumps is compared with the heat load of the total fuel assembly over the same decay time. The same process is then repeated for the masses plus one standard deviation of the isotopic uncertainties which propagates the uncertainty associated with each separation time. Table 3.4 shows these decays heat loads for the first 1000 years and Table 3.5 shows 2,500 to 10,000 years (see the Appendix A for a more detailed description of this model). As data shows, the majority of the long term heat load resides with the decaying of actinides. If these can be burned off in some reprocessing scheme, margin to the taxing heat limits on the repository could be realized. This experiment provides some insight for the more rigorous experiment of uncertainty propagation in recycled fast reactor fuel.

\begin{tabular}{|c|c|c|c|c|c|c|}
\hline Nuclide & Mass at & 5 Years & Mass at & 0 Years & Mass at & 5 Years \\
\hline & Grams & $+/-$ & Grams & $+/-$ & Grams & $+/-$ \\
\hline u234 & $1.60 \mathrm{E}+02$ & $.53 E+00$ & 1.67E+02 & $2.64 \mathrm{E}+00$ & & \\
\hline $\mathrm{u} 235$ & $1.23 E+04$ & $1.62 \mathrm{E}+02$ & & & & \\
\hline u236 & $5.53 E+03$ & $3.94 \mathrm{E}+01$ & $5.53 E+03$ & & $5.53 E+03$ & $3.94 \mathrm{E}+01$ \\
\hline u237 & & $9.45 \mathrm{E}-07$ & & & & \\
\hline u238 & & & & & & \\
\hline np237 & & +00 & & & -02 & $E+00$ \\
\hline np239 & E-04 & 1.37E-05 & E-04 & & $=-05$ & 1.36E-05 \\
\hline pu2 & & & & & +02 & +00 \\
\hline pu239 & -03 & +01 & +03 & +01 & $=03$ & $E+01$ \\
\hline pu240 & & $=01$ & +03 & & +03 & $E+01$ \\
\hline pu241 & +03 & $E+01$ & $E+02$ & $E+01$ & +02 & $E+01$ \\
\hline pu242 & $5.58 \mathrm{E}+02$ & $1.45 \mathrm{E}+01$ & $5.58 \mathrm{E}+02$ & $1.45 \mathrm{E}+01$ & $5.58 \mathrm{E}+02$ & $1.45 \mathrm{E}+01$ \\
\hline am241 & $3.79 \mathrm{E}+02$ & $9.49 \mathrm{E}+00$ & $6.41 \mathrm{E}+02$ & $0 \mathrm{E}+01$ & $2 E+03$ & $2.80 \mathrm{E}+01$ \\
\hline am242m & & & & & E-01 & $E-02$ \\
\hline am243 & $1.16 \mathrm{E}+02$ & $1.59 \mathrm{E}+01$ & 1.16E+02 & $1.59 \mathrm{E}+01$ & 1.16E+02 & $1.58 \mathrm{E}+01$ \\
\hline cm242 & 8.07E-03 & 1.75E-04 & $2.28 \mathrm{E}-03$ & 4.96E-05 & 2.12E-03 & 4.60E-05 \\
\hline $\mathrm{cm} 244$ & $2.66 \mathrm{E}+01$ & $3.02 E+00$ & $2.20 \mathrm{E}+01$ & $2.49 \mathrm{E}+00$ & $1.24 \mathrm{E}+01$ & $1.40 \mathrm{E}+00$ \\
\hline $\mathrm{cm} 245$ & $1.21 \mathrm{E}+00$ & 1.24E-01 & $1.21 \mathrm{E}+00$ & $1.24 \mathrm{E}-01$ & $1.21 \mathrm{E}+00$ & 1.24E-01 \\
\hline
\end{tabular}

Table 3.3: Masses, with uncertainty, of actinides at 3 decay times. 


\begin{tabular}{|c|c|c|c|c|c|c|c|c|}
\hline \multirow{3}{*}{ Element } & \multicolumn{8}{|c|}{$\begin{array}{l}\text { If Separated At } 5 \text { Years After Discharge, a } 4.5 \text { w/o Burned for } 40 \text { GWD/MTU, } \\
\text { (Note: times below are after separation) }\end{array}$} \\
\hline & \multicolumn{2}{|c|}{$50(55)$ Years } & \multicolumn{2}{|c|}{$100(105)$ Years } & \multicolumn{2}{|c|}{$500(505)$ Years } & \multicolumn{2}{|c|}{1000 (1005) Years } \\
\hline & w & $+/-W^{\prime}$ & $\mathbf{W}$ & $+/-W^{\prime}$ & $\mathbf{W}$ & $+/-W^{\prime}$ & $\mathbf{W}$ & $+/-W^{\prime}$ \\
\hline $\bar{U}$ & 0.049 & 0.001 & 0.049 & 0.001 & 0.049 & 0.001 & 0.049 & 0.001 \\
\hline $\mathrm{Np}$ & 0.012 & 0.000 & 0.012 & 0.000 & 0.012 & 0.000 & 0.013 & 0.000 \\
\hline $\mathrm{Pu}$ & 218.010 & 4.235 & 195.781 & 4.025 & 91.127 & 2.085 & 52.097 & 1.136 \\
\hline $\mathrm{Am}$ & 41.217 & 1.102 & 38.110 & 1.026 & 20.348 & 0.589 & 9.513 & 0.319 \\
\hline $\mathrm{Cm}$ & 11.264 & 0.310 & 1.823 & 0.050 & 0.186 & 0.005 & 0.179 & 0.005 \\
\hline
\end{tabular}

\begin{tabular}{|c|c|c|c|c|c|c|c|c|}
\hline \multirow{3}{*}{ Element } & \multicolumn{8}{|c|}{$\begin{array}{l}\text { If Separated At } 10 \text { Years After Discharge, a } 4.5 \text { w/o Burned for } 40 \text { GWD/MTU, } \\
\text { (Note: times below are after separation) }\end{array}$} \\
\hline & \multicolumn{2}{|c|}{$50(60)$ Years } & \multicolumn{2}{|c|}{100 (110) Years } & \multicolumn{2}{|c|}{$500(510)$ Years } & \multicolumn{2}{|c|}{$1000(1010)$ Years } \\
\hline & $\mathbf{W}$ & $+/-W^{\prime}$ & $\mathbf{W}$ & $+1-W^{\prime}$ & $\mathbf{W}$ & $+/-W^{\prime}$ & $\mathbf{W}$ & $+/-W^{\prime}$ \\
\hline $\mathrm{U}$ & 0.050 & 0.001 & 0.050 & 0.001 & 0.050 & 0.001 & 0.051 & 0.001 \\
\hline $\mathrm{Np}$ & 0.013 & 0.000 & 0.013 & 0.000 & 0.013 & 0.000 & 0.013 & 0.000 \\
\hline $\mathrm{Pu}$ & 188.917 & 3.590 & 167.357 & 3.374 & 76.953 & 1.739 & 45.779 & 0.981 \\
\hline $\mathrm{Am}$ & 68.819 & 1.792 & 63.588 & 1.662 & 33.770 & 0.925 & 15.538 & 0.471 \\
\hline $\mathrm{Cm}$ & 9.302 & 1.033 & 1.506 & 0.167 & 0.156 & 0.016 & 0.149 & 0.015 \\
\hline
\end{tabular}

\begin{tabular}{|c|c|c|c|c|c|c|c|c|}
\hline \multirow{3}{*}{ Element } & \multicolumn{8}{|c|}{$\begin{array}{l}\text { If Separated At } 25 \text { Years After Discharge, a } 4.5 \text { w/o Burned for } 40 \text { GWD/MTU, } \\
\text { (Note: times below are after separation) }\end{array}$} \\
\hline & \multicolumn{2}{|c|}{$50(75)$ Years } & \multicolumn{2}{|c|}{$100(125)$ Years } & \multicolumn{2}{|c|}{500 (525) Years } & \multicolumn{2}{|c|}{1000 (1025) Years } \\
\hline & $\mathbf{W}$ & $+/-W^{\prime}$ & $\mathbf{W}$ & $+/-W^{\prime}$ & $\mathbf{W}$ & $+/-W^{\prime}$ & $\mathbf{W}$ & $+/-W^{\prime}$ \\
\hline$U$ & 0.054 & 0.001 & 0.054 & 0.001 & 0.054 & 0.001 & 0.054 & 0.001 \\
\hline $\mathrm{Np}$ & 0.013 & 0.000 & 0.013 & 0.000 & 0.013 & 0.000 & 0.013 & 0.000 \\
\hline $\mathrm{Pu}$ & 131.727 & 1.327 & 112.187 & 1.425 & 50.156 & 1.033 & 33.859 & 0.677 \\
\hline $\mathrm{Am}$ & 119.336 & 3.064 & 110.214 & 2.835 & 58.356 & 1.542 & 26.584 & 0.747 \\
\hline $\mathrm{Cm}$ & 5.274 & 0.516 & 0.857 & 0.083 & 0.093 & 0.008 & 0.090 & 0.008 \\
\hline
\end{tabular}

\begin{tabular}{|c|c|c|c|c|c|c|c|c|}
\hline & \multicolumn{8}{|c|}{$\begin{array}{l}\text { 1MT of Fuel After Discharge, a } 4.5 \text { w/o FA Burned for } 40 \text { GWD/MTU, (Note: times } \\
\text { below are after irradiation) }\end{array}$} \\
\hline & \multicolumn{2}{|c|}{50 Years } & \multicolumn{2}{|c|}{ 100Years } & \multicolumn{2}{|c|}{500 Years } & \multicolumn{2}{|c|}{1000 Years } \\
\hline & $\mathbf{W}$ & $+/-W^{\prime}$ & $\mathbf{W}$ & $+/-W^{\prime}$ & $\mathbf{W}$ & $+/-W^{\prime}$ & $\mathbf{W}$ & $+/-W^{\prime}$ \\
\hline FUEL & 662.820 & 19.380 & 356.663 & 8.038 & 112.558 & 4.262 & 62.215 & 1.999 \\
\hline
\end{tabular}

Table 3.4: Comparison of separation at 3 times vs. no separation, first 1000 years of decay. 


\begin{tabular}{|c|c|c|c|c|c|c|}
\hline \multirow{3}{*}{ Element } & \multicolumn{6}{|c|}{$\begin{array}{l}\text { If Separated At } 5 \text { Years After Discharge, a } 4.5 \text { w/o FA } \\
\text { Burned for } 40 \text { GWD/MTU, (Note: times below are after } \\
\text { separation) }\end{array}$} \\
\hline & \multicolumn{2}{|c|}{$2500(2505)$ Years } & \multicolumn{2}{|c|}{5000 (5005) Years } & \multicolumn{2}{|c|}{$\begin{array}{c}10000(10005) \\
\text { Years }\end{array}$} \\
\hline & $\mathbf{W}$ & $+/-W^{\prime}$ & $\mathbf{W}$ & $+/-W^{\prime}$ & $\mathbf{W}$ & $+/-W^{\prime}$ \\
\hline $\mathrm{U}$ & 0.051 & 0.001 & 0.056 & 0.001 & 0.065 & 0.001 \\
\hline $\mathrm{Np}$ & 0.013 & 0.000 & 0.013 & 0.000 & 0.014 & 0.000 \\
\hline $\mathrm{Pu}$ & 23.033 & 0.413 & 17.347 & 0.281 & 12.833 & 0.187 \\
\hline $\mathrm{Am}$ & 1.481 & 0.112 & 0.604 & 0.079 & 0.439 & 0.059 \\
\hline $\mathrm{Cm}$ & 0.154 & 0.004 & 0.119 & 0.003 & 0.071 & 0.002 \\
\hline
\end{tabular}

\begin{tabular}{|c|c|c|c|c|c|c|}
\hline \multirow{3}{*}{ Element } & \multicolumn{6}{|c|}{$\begin{array}{l}\text { If Separated At } 10 \text { Years After Discharge, a } 4.5 \text { w/o FA } \\
\text { Burned for } 40 \text { GWD/MTU, (Note: times below are after } \\
\text { separation) }\end{array}$} \\
\hline & \multicolumn{2}{|c|}{$2500(2510)$ Years } & \multicolumn{2}{|c|}{$5000(5010)$ Years } & \multicolumn{2}{|c|}{$\begin{array}{c}10000(10010) \\
\text { Years }\end{array}$} \\
\hline & $\mathbf{W}$ & $+/-W^{\prime}$ & $\mathbf{W}$ & $+/-W^{\prime}$ & $\mathbf{W}$ & $+/-W^{\prime}$ \\
\hline$U$ & 0.053 & 0.001 & 0.057 & 0.001 & 0.067 & 0.001 \\
\hline $\mathrm{Np}$ & 0.013 & 0.000 & 0.013 & 0.000 & 0.014 & 0.000 \\
\hline $\mathrm{Pu}$ & 22.472 & 0.399 & 17.343 & 0.280 & 12.833 & 0.187 \\
\hline $\mathrm{Am}$ & 2.029 & 0.128 & 0.619 & 0.081 & 0.444 & 0.060 \\
\hline $\mathrm{Cm}$ & 0.129 & 0.013 & 0.100 & 0.010 & 0.060 & 0.006 \\
\hline
\end{tabular}

\begin{tabular}{|c|c|c|c|c|c|c|}
\hline \multirow{3}{*}{ Element } & \multicolumn{6}{|c|}{$\begin{array}{l}\text { If Separated At } 25 \text { Years After Discharge, a } 4.5 \text { w/o FA } \\
\text { Burned for } 40 \text { GWD/MTU, (Note: times below are after } \\
\text { separation) }\end{array}$} \\
\hline & \multicolumn{2}{|c|}{2500 (2525) Years } & \multicolumn{2}{|c|}{5000 (5025) Years } & \multicolumn{2}{|c|}{$\begin{array}{c}10000(10025) \\
\text { Years }\end{array}$} \\
\hline & W & $+/-W^{\prime}$ & $\mathbf{W}$ & $+/-W^{\prime}$ & $\mathbf{W}$ & $+/-W^{\prime}$ \\
\hline $\mathrm{U}$ & 0.056 & 0.001 & 0.061 & 0.001 & 0.072 & 0.001 \\
\hline $\mathrm{Np}$ & 0.013 & 0.000 & 0.014 & 0.000 & 0.015 & 0.000 \\
\hline $\mathrm{Pu}$ & 21.410 & 0.372 & 17.332 & 0.280 & 12.828 & 0.186 \\
\hline $\mathrm{Am}$ & 3.037 & 0.151 & 0.647 & 0.081 & 0.456 & 0.059 \\
\hline $\mathrm{Cm}$ & 0.078 & 0.007 & 0.061 & 0.005 & 0.036 & 0.003 \\
\hline
\end{tabular}

\begin{tabular}{|l|c|c|c|c|c|c|}
\hline \multirow{3}{*}{} & \multicolumn{6}{|c|}{ 1MT of Fuel After Discharge, a 4.5 w/o FA Burned for 40} \\
& GWD/MTU, (Note: times below are after irradiation) \\
\cline { 2 - 7 } & $\mathbf{2 5 0 0}$ Years & \multicolumn{2}{|c|}{$\mathbf{5 0 0 0}$ Years } & $\mathbf{1 0 0 0 0}$ Years \\
\cline { 2 - 7 } & $\mathbf{W}$ & $\mathbf{+}-\mathbf{W}$ & $\mathbf{W}$ & $\mathbf{+}-\mathbf{W}$ & $\mathbf{W}$ & $\mathbf{+ / - \mathbf { W }}$ \\
\hline FUEL & 24.808 & 0.594 & 18.184 & 0.446 & 13.460 & 0.281 \\
\hline
\end{tabular}

Table 3.5: Comparison of separation at 3 times vs. no separation, 2500 - 10,000 years of decay. 


\subsubsection{Typical LWR with UOX Fuel}

The SCALE package comes with a prepared "test" card-image cross-section library that is ideally representative of a typical LWR. The following data depict a uranium oxide fuel depleted, with stochastic sampling, in ORIGEN using this typical LWR cross section library provided with SCALE, and the typical LWR flux spectrum (also provided) to collapse the 44-group covariance library. The UOX fuel is $4.5 \mathrm{w} / \mathrm{o}$ and burned to $40 \mathrm{GWD} / \mathrm{MTU}$ in a single representative burnup step divided into depletion intervals by default in ORIGEN (see the Appendix A for a more detailed description of these models). While in terms of isotopics, the model produced similar results to the PWR model as expected (Table 3.6), the uncertainties using the typical flux spectrum tended to over predict those obtained from the PWR model (Table 3.7).

\begin{tabular}{|c|c|c|c|c|c|}
\hline \multicolumn{6}{|c|}{ Discharge Isotopics, grams / MTHM } \\
\hline pb210 & $2.113 \mathrm{E}-11$ & np237 & $4.696 \mathrm{E}+02$ & cm244 & $1.930 \mathrm{E}+01$ \\
\hline ra226 & $641 \mathrm{E}-08$ & np239 & & cm245 & 7.951E-01 \\
\hline ac227 & 114E-09 & pu238 & $1.343 E+02$ & c 14 & $\mathrm{E}-03$ \\
\hline th227 & & & & & +00 \\
\hline th230 & 1.164E-03 & pu240 & $E+03$ & 90 & $E+02$ \\
\hline pa231 & 3.150 & pu241 & $E+03$ & & $9.754 \mathrm{E}+02$ \\
\hline u234 & 4 & & +02 & & +02 \\
\hline u235 & $1.547 \mathrm{E}+02$ & am241 & $3.190 \mathrm{E}+01$ & cs137 & +03 \\
\hline $\mathbf{u} 236$ & $5.641 \mathrm{E}+03$ & am242m & 5.701E-01 & ba137m & $5 \mathrm{E}-04$ \\
\hline u237 & $1.202 \mathrm{E}+01$ & am243 & $8.028 \mathrm{E}+01$ & y90 & 1.939E-01 \\
\hline u238 & $9.323 E+05$ & cm242 & $1.000 \mathrm{E}+01$ & cs134 & $1.506 \mathrm{E}+02$ \\
\hline
\end{tabular}

Table 3.6: Discharge isotopics for typical LWR simplified model. 


\begin{tabular}{|c|c|c|c|c|c|}
\hline \multicolumn{7}{|c|}{ Isotopic Uncertainties (\% St. Dev.) } \\
\hline Nuclide & Uncertainty & Nuclide & Uncertainty & Nuclide & Uncertainty \\
\hline pb210 & 1.1518 & np237 & 1.0320 & cm244 & 11.4636 \\
\hline ra226 & 1.3188 & np239 & 12.6984 & cm245 & 11.2314 \\
\hline ac227 & 0.8004 & pu238 & 2.4674 & c 14 & 0.6833 \\
\hline th227 & 0.8007 & pu239 & 2.2019 & se 79 & 0.4147 \\
\hline th230 & 1.4124 & pu240 & 2.8370 & sr 90 & 0.7126 \\
\hline pa231 & 1.3595 & pu241 & 3.7081 & tc 99 & 1.6437 \\
\hline u234 & 1.4532 & pu242 & 3.9948 & i129 & 0.5690 \\
\hline u235 & 6.1838 & am241 & 3.6751 & cs137 & 0.3347 \\
\hline u236 & 1.3642 & am242m & 3.1688 & ba137m & 0.3347 \\
\hline u237 & 3.7081 & am243 & 12.6985 & y 90 & 0.7126 \\
\hline u238 & 0.0894 & $\mathbf{c m 2 4 2}$ & 3.1693 & cs134 & 1.9556 \\
\hline
\end{tabular}

Table 3.7: Isotopics uncertainties for typical LWR simplified model.

\subsubsection{BWR Models with UOX Fuel}

The following data are for a representative UOX fuel burned in a boiling water reactor. An updated cross-section library is created using SAS2H for one representative burnup step, and the 1-group working library used with ORIGEN. The 44-group covariance library is collapsed to 1-group using the beginning of cycle flux spectrum for this fuel, and stochastic sampling is implemented. The UOX fuel is $4.5 \mathrm{w} / \mathrm{o}$ and burned to $40 \mathrm{GWD} / \mathrm{MTU}$ in a single cycle representative of a once-through fuel. The geometry is a 7x7 General Electric fuel assembly homogenized to $4.5 \mathrm{w} / \mathrm{o}$, with no burnable poison elements; adapted from Hermann [32]. The experiment is repeated for void fractions of $0 \%, 35 \%, 50 \%$, and $65 \%$ by modifying the average density of the coolant (see the Appendix A for a more detailed description of this model). Table 3.8 - Table 3.11 show the discharge isotopics for each of the voids, and Table 3.12 gives a listing of the isotopics uncertainties for each void. The key observation to take away from these data, as will be stressed again later, that for UOX fuels in a LWR (be it PWR or BWR), the uncertainties are on the same order of magnitude. Across the range of voids, uncertainties do change by a factor of 1.1 to 1.7 . The 
top of the fuel is typically at $70-80 \%$ void while the bottom is always at $0 \%$ void, resulting in an average operating void in the 40\% - 50\% range. This indicates that the isotopics and their uncertainties will be a function of not only burnup but also void history, both dependent upon not only the fuel assembly, but axial position within the assembly. The BWR results in that voided region are similar to the PWR results. In fact the PWR uncertainties fall within the uncertainties of the $50 \%$ void and $65 \%$ BWR void results.

\begin{tabular}{|l|r|l|r|l|r|}
\hline \multicolumn{7}{|c|}{ Discharge Isotopics, grams / MTHM } \\
\hline pb210 & $1.338 \mathrm{E}-11$ & np237 & $3.433 \mathrm{E}+02$ & cm244 & $9.658 \mathrm{E}+00$ \\
\hline ra226 & $1.793 \mathrm{E}-08$ & np239 & $8.156 \mathrm{E}+01$ & cm245 & $1.883 \mathrm{E}-01$ \\
\hline ac227 & $1.680 \mathrm{E}-09$ & pu238 & $8.692 \mathrm{E}+01$ & c 14 & $3.165 \mathrm{E}-03$ \\
\hline th227 & $4.465 \mathrm{E}-12$ & pu239 & $3.294 \mathrm{E}+03$ & se 79 & $5.966 \mathrm{E}+00$ \\
\hline th230 & $1.380 \mathrm{E}-03$ & pu240 & $1.848 \mathrm{E}+03$ & sr 90 & $7.468 \mathrm{E}+02$ \\
\hline pa231 & $1.941 \mathrm{E}-04$ & pu241 & $8.414 \mathrm{E}+02$ & tc 99 & $1.001 \mathrm{E}+03$ \\
\hline u234 & $1.675 \mathrm{E}+02$ & pu242 & $4.334 \mathrm{E}+02$ & i129 & $1.602 \mathrm{E}+02$ \\
\hline u235 & $8.102 \mathrm{E}+03$ & am241 & $1.936 \mathrm{E}+01$ & cs137 & $1.491 \mathrm{E}+03$ \\
\hline u236 & $5.706 \mathrm{E}+03$ & am242m & $3.008 \mathrm{E}-01$ & ba137m & $2.290 \mathrm{E}-04$ \\
\hline u237 & $9.736 \mathrm{E}+00$ & am243 & $5.414 \mathrm{E}+01$ & y90 & $2.063 \mathrm{E}-01$ \\
\hline u238 & $9.370 \mathrm{E}+05$ & $\mathbf{c m 2 4 2}$ & $7.232 \mathrm{E}+00$ & cs134 & $1.141 \mathrm{E}+02$ \\
\hline
\end{tabular}

Table 3.8: Discharge isotopics for BWR fuel burned at $0 \%$ void.

\begin{tabular}{|l|c|l|l|l|r|}
\hline \multicolumn{7}{|c|}{ Discharge Isotopics, grams / MTHM } \\
\hline pb210 & $1.692 \mathrm{E}-11$ & np237 & $4.265 \mathrm{E}+02$ & cm244 & $1.668 \mathrm{E}+01$ \\
\hline ra226 & $1.728 \mathrm{E}-08$ & np239 & $8.489 \mathrm{E}+01$ & cm245 & $4.522 \mathrm{E}-01$ \\
\hline ac227 & $2.391 \mathrm{E}-09$ & pu238 & $1.202 \mathrm{E}+02$ & c 14 & $3.247 \mathrm{E}-03$ \\
\hline th227 & $6.618 \mathrm{E}-12$ & pu239 & $4.161 \mathrm{E}+03$ & se 79 & $5.911 \mathrm{E}+00$ \\
\hline th230 & $1.274 \mathrm{E}-03$ & pu240 & $1.922 \mathrm{E}+03$ & sr 90 & $7.234 \mathrm{E}+02$ \\
\hline pa231 & $2.459 \mathrm{E}-04$ & pu241 & $1.112 \mathrm{E}+03$ & tc 99 & $9.833 \mathrm{E}+02$ \\
\hline $\mathbf{u 2 3 4}$ & $1.613 \mathrm{E}+02$ & pu242 & $4.859 \mathrm{E}+02$ & i129 & $1.663 \mathrm{E}+02$ \\
\hline $\mathbf{u 2 3 5}$ & $9.843 \mathrm{E}+03$ & am241 & $2.770 \mathrm{E}+01$ & cs137 & $1.489 \mathrm{E}+03$ \\
\hline $\mathbf{u 2 3 6}$ & $5.640 \mathrm{E}+03$ & am242m & $4.901 \mathrm{E}-01$ & ba137m & $2.288 \mathrm{E}-04$ \\
\hline $\mathbf{u 2 3 7}$ & $1.124 \mathrm{E}+01$ & $\mathbf{a m 2 4 3}$ & $7.594 \mathrm{E}+01$ & y90 & $1.977 \mathrm{E}-01$ \\
\hline $\mathbf{u 2 3 8}$ & $9.340 \mathrm{E}+05$ & $\mathbf{c m 2 4 2}$ & $9.467 \mathrm{E}+00$ & $\mathbf{c s 1 3 4}$ & $1.268 \mathrm{E}+02$ \\
\hline
\end{tabular}

Table 3.9: Discharge isotopics for BWR fuel burned at $35 \%$ void. 


\begin{tabular}{|c|c|c|c|c|c|}
\hline \multicolumn{6}{|c|}{ Discharge Isotopics, grams / MTHM } \\
\hline pb210 & $2.010 \mathrm{E}-11$ & np237 & $4.842 \mathrm{E}+02$ & cm244 & $2.278 \mathrm{E}+01$ \\
\hline ra226 & 1.671E-08 & np239 & $8.780 \mathrm{E}+01$ & $\mathrm{~cm} 245$ & $7.550 \mathrm{E}-01$ \\
\hline ac227 & $.958 \mathrm{E}-$ & pu238 & 02 & c 14 & 3.306 \\
\hline th227 & 8.384E-12 & pu239 & $E+03$ & se 79 & $5.867 E+00$ \\
\hline th230 & 1.195E-03 & pu240 & $1.990 \mathrm{E}+03$ & sr 90 & $\mathrm{E}+02$ \\
\hline pa231 & 2.839E-04 & pu241 & 1.33 & tc 99 & 9.68 \\
\hline u234 & $1.564 \mathrm{E}+02$ & pu242 & 5.166 & $\mathbf{i 1 2 9}$ & +02 \\
\hline u235 & $1.109 \mathrm{E}+04$ & am241 & $3.478 \mathrm{E}+01$ & cs137 & $1.487 E+03$ \\
\hline u236 & $5.621 \mathrm{E}+03$ & am242m & $6.776 \mathrm{E}-01$ & ba137m & $2.286 \mathrm{E}-04$ \\
\hline u237 & $1.223 \mathrm{E}+01$ & am243 & $9.138 \mathrm{E}+01$ & y90 & $1.918 \mathrm{E}-01$ \\
\hline u238 & $9.317 \mathrm{E}+05$ & cm242 & $1.122 \mathrm{E}+01$ & cs134 & $1.359 E+02$ \\
\hline
\end{tabular}

Table 3.10: Discharge isotopics for BWR fuel burned at $50 \%$ void.

\begin{tabular}{|l|l|l|l|l|r|}
\hline \multicolumn{7}{|c|}{ Discharge Isotopics, grams / MTHM } \\
\hline pb210 & $2.601 \mathrm{E}-11$ & np237 & $5.685 \mathrm{E}+02$ & cm244 & $3.264 \mathrm{E}+01$ \\
\hline ra226 & $1.570 \mathrm{E}-08$ & np239 & $9.288 \mathrm{E}+01$ & cm245 & $1.398 \mathrm{E}+00$ \\
\hline ac227 & $3.847 \mathrm{E}-09$ & pu238 & $1.934 \mathrm{E}+02$ & c 14 & $3.392 \mathrm{E}-03$ \\
\hline th227 & $1.126 \mathrm{E}-11$ & pu239 & $6.262 \mathrm{E}+03$ & se 79 & $5.797 \mathrm{E}+00$ \\
\hline th230 & $1.073 \mathrm{E}-03$ & pu240 & $2.119 \mathrm{E}+03$ & sr 90 & $6.803 \mathrm{E}+02$ \\
\hline pa231 & $3.400 \mathrm{E}-04$ & pu241 & $1.704 \mathrm{E}+03$ & tc 99 & $9.429 \mathrm{E}+02$ \\
\hline u234 & $1.487 \mathrm{E}+02$ & pu242 & $5.478 \mathrm{E}+02$ & i129 & $1.764 \mathrm{E}+02$ \\
\hline u235 & $1.290 \mathrm{E}+04$ & am241 & $4.697 \mathrm{E}+01$ & cs137 & $1.483 \mathrm{E}+03$ \\
\hline u236 & $5.638 \mathrm{E}+03$ & am242m & $1.058 \mathrm{E}+00$ & ba137m & $2.281 \mathrm{E}-04$ \\
\hline u237 & $1.363 \mathrm{E}+01$ & am243 & $1.118 \mathrm{E}+02$ & y90 & $1.837 \mathrm{E}-01$ \\
\hline u238 & $9.280 \mathrm{E}+05$ & cm242 & $1.392 \mathrm{E}+01$ & cs134 & $1.488 \mathrm{E}+02$ \\
\hline
\end{tabular}

Table 3.11: Discharge isotopics for BWR fuel burned at $65 \%$ void. 


\begin{tabular}{|l|c|c|c|c|}
\hline \multicolumn{5}{|c|}{ Isotopic Uncertainties (\% St. Dev.) } \\
\hline \multirow{2}{*}{ Nuclide } & \multicolumn{4}{|c|}{ Uncertainty } \\
\cline { 2 - 5 } & $\mathbf{0 \%}$ Void & $\mathbf{3 5 \%}$ Void & $\mathbf{5 0 \%}$ Void & $\mathbf{6 5 \%}$ Void \\
\hline pb210 & 0.798 & 1.056 & 1.126 & 1.388 \\
\hline ra226 & 0.902 & 1.193 & 1.274 & 1.575 \\
\hline ac227 & 0.335 & 0.429 & 0.477 & 0.548 \\
\hline th227 & 0.335 & 0.429 & 0.477 & 0.548 \\
\hline th230 & 1.022 & 1.335 & 1.415 & 1.725 \\
\hline pa231 & 0.424 & 0.504 & 0.570 & 0.594 \\
\hline u234 & 1.146 & 1.462 & 1.526 & 1.809 \\
\hline u235 & 1.360 & 1.472 & 1.757 & 1.677 \\
\hline u236 & 0.481 & 0.608 & 0.731 & 0.839 \\
\hline u237 & 2.105 & 2.390 & 2.492 & 2.479 \\
\hline u238 & 0.055 & 0.065 & 0.069 & 0.079 \\
\hline np237 & 0.541 & 0.593 & 0.638 & 0.697 \\
\hline np239 & 8.910 & 11.086 & 12.685 & 14.483 \\
\hline pu238 & 1.102 & 1.101 & 1.147 & 1.172 \\
\hline pu239 & 0.817 & 0.867 & 0.874 & 0.920 \\
\hline pu240 & 2.157 & 2.381 & 2.637 & 2.536 \\
\hline pu241 & 2.105 & 2.390 & 2.492 & 2.479 \\
\hline pu242 & 2.011 & 2.252 & 2.437 & 2.531 \\
\hline am241 & 2.089 & 2.372 & 2.474 & 2.462 \\
\hline am242m & 1.807 & 2.071 & 2.156 & 2.154 \\
\hline am243 & 8.910 & 11.086 & 12.685 & 14.483 \\
\hline cm242 & 1.806 & 2.070 & 2.155 & 2.153 \\
\hline cm244 & 7.416 & 9.233 & 10.618 & 12.041 \\
\hline cm245 & 6.816 & 8.431 & 9.687 & 10.851 \\
\hline c 14 & 0.392 & 0.441 & 0.483 & 0.544 \\
\hline se 79 & 0.316 & 0.363 & 0.408 & 0.465 \\
\hline sr 90 & 0.322 & 0.381 & 0.436 & 0.496 \\
\hline tc 99 & 1.309 & 1.609 & 1.864 & 2.238 \\
\hline i129 & 0.362 & 0.408 & 0.444 & 0.502 \\
\hline cs137 & 0.326 & 0.374 & 0.413 & 0.473 \\
\hline ba137m & 0.326 & 0.374 & 0.413 & 0.473 \\
\hline y 90 & 0.322 & 0.381 & 0.436 & 0.496 \\
\hline cs134 & 0.962 & 1.063 & 1.166 & 1.305 \\
\hline
\end{tabular}

Table 3.12: Isotopics uncertainties for BWR models.

\subsubsection{PWR Models with MOX Fuels}

The following data is for two representative MOX fuels burned in a pressurized water reactor, adapted from fuel compositions in Bathke [4]. An updated cross-section library is created using SAS2H for one representative burnup step and the 1-group binary library used 
with ORIGEN. The 44-group covariance library is collapsed to 1-group using the beginning of cycle flux spectrum for each fuel, and stochastic sampling is implemented. The first, clean MOX fuel is $91.903 \mathrm{w} / \mathrm{o}$ uranium with the following composition: $1.40 \mathrm{w} / \mathrm{o}$ U-235, 98.572 w/o U-238, $0.028 \mathrm{w} / \mathrm{o}$ U-234, and $8.097 \mathrm{w} / \mathrm{o}$ plutonium. The plutonium has the composition: 1.655 w/o Pu-238, 61.751 w/o Pu-239, 24.701 w/o Pu-240, 3.248 w/o Pu-241, 8.645 w/o Pu242. The second MOX fuel is representative of imperfect separation techniques and includes only 89.403 w/o uranium and impurities of 1 w/o Np-237 and 1.5 w/o Am-241. The geometry of both fuels is that of a $17 \times 17$ Westinghouse-type fuel assembly with 25 water holes and they are each burned to $50 \mathrm{GWD} / \mathrm{MTHM}$ (see the Appendix A for a more detailed description of these models). Table 3.13 and Table 3.14 show the discharge isotopics for the two MOX fuels, and Table 3.15 presents the isotopic uncertainties for the two fuels. It can be seen from these data that making a significant change in the fuel composition will result in a change in the uncertainties. 


\begin{tabular}{|l|l|l|l|l|r|}
\hline \multicolumn{7}{|c|}{ Discharge Isotopics, grams / MTHM } \\
\hline pb210 & $1.310 \mathrm{E}-10$ & np237 & $3.290 \mathrm{E}+02$ & cm244 & $9.958 \mathrm{E}+02$ \\
\hline ra226 & $1.840 \mathrm{E}-08$ & np239 & $9.836 \mathrm{E}+01$ & cm245 & $9.472 \mathrm{E}+01$ \\
\hline ac227 & $6.215 \mathrm{E}-09$ & pu238 & $4.530 \mathrm{E}+03$ & c 14 & $5.611 \mathrm{E}-03$ \\
\hline th227 & $3.415 \mathrm{E}-11$ & pu239 & $2.384 \mathrm{E}+04$ & se 79 & $6.108 \mathrm{E}+00$ \\
\hline th230 & $1.292 \mathrm{E}-03$ & pu240 & $1.549 \mathrm{E}+04$ & sr 90 & $4.569 \mathrm{E}+02$ \\
\hline pa231 & $4.894 \mathrm{E}-04$ & pu241 & $9.475 \mathrm{E}+03$ & tc 99 & $1.127 \mathrm{E}+03$ \\
\hline $\mathbf{u 2 3 4}$ & $2.299 \mathrm{E}+02$ & pu242 & $6.822 \mathrm{E}+03$ & i129 & $3.038 \mathrm{E}+02$ \\
\hline $\mathbf{u 2 3 5}$ & $7.081 \mathrm{E}+03$ & am241 & $6.046 \mathrm{E}+02$ & cs137 & $1.864 \mathrm{E}+03$ \\
\hline $\mathbf{u 2 3 6}$ & $1.503 \mathrm{E}+03$ & am242m & $1.961 \mathrm{E}+01$ & ba137m & $2.872 \mathrm{E}-04$ \\
\hline $\mathbf{u 2 3 7}$ & $7.047 \mathrm{E}+00$ & am243 & $1.851 \mathrm{E}+03$ & y90 & $1.218 \mathrm{E}-01$ \\
\hline $\mathbf{u 2 3 8}$ & $8.759 \mathrm{E}+05$ & $\mathbf{c m 2 4 2}$ & $1.355 \mathrm{E}+02$ & cs134 & $2.055 \mathrm{E}+02$ \\
\hline
\end{tabular}

Table 3.13: Discharge isotopics for the "clean" MOX fuel.

\begin{tabular}{|l|l|l|l|l|r|}
\hline \multicolumn{7}{|c|}{ Discharge Isotopics, grams / MTHM } \\
\hline pb210 & $1.442 \mathrm{E}-09$ & np237 & $5.422 \mathrm{E}+03$ & cm244 & $1.075 \mathrm{E}+03$ \\
\hline ra226 & $1.928 \mathrm{E}-08$ & np239 & $9.590 \mathrm{E}+01$ & cm245 & $1.075 \mathrm{E}+02$ \\
\hline ac227 & $6.988 \mathrm{E}-09$ & pu238 & $1.328 \mathrm{E}+04$ & c 14 & $5.300 \mathrm{E}-03$ \\
\hline th227 & $2.498 \mathrm{E}-10$ & pu239 & $2.816 \mathrm{E}+04$ & se 79 & $6.053 \mathrm{E}+00$ \\
\hline th230 & $1.441 \mathrm{E}-03$ & pu240 & $1.593 \mathrm{E}+04$ & sr 90 & $4.491 \mathrm{E}+02$ \\
\hline pa231 & $5.488 \mathrm{E}-04$ & pu241 & $9.545 \mathrm{E}+03$ & tc 99 & $1.105 \mathrm{E}+03$ \\
\hline u234 & $3.133 \mathrm{E}+02$ & pu242 & $7.792 \mathrm{E}+03$ & i129 & $3.039 \mathrm{E}+02$ \\
\hline u235 & $7.406 \mathrm{E}+03$ & am241 & $4.880 \mathrm{E}+03$ & cs137 & $1.843 \mathrm{E}+03$ \\
\hline u236 & $1.501 \mathrm{E}+03$ & am242m & $2.003 \mathrm{E}+02$ & ba137m & $2.842 \mathrm{E}-04$ \\
\hline $\mathbf{u 2 3 7}$ & $6.927 \mathrm{E}+00$ & am243 & $2.130 \mathrm{E}+03$ & y90 & $1.196 \mathrm{E}-01$ \\
\hline u238 & $8.501 \mathrm{E}+05$ & $\mathbf{c m 2 4 2}$ & $1.304 \mathrm{E}+03$ & cs134 & $1.993 \mathrm{E}+02$ \\
\hline
\end{tabular}

Table 3.14: Discharge isotopics for MOX fuel with impurities. 


\begin{tabular}{|l|c|c|}
\hline \multicolumn{3}{|c|}{ Isotopic Uncertainties (\% St. Dev.) } \\
\hline \multirow{2}{*}{ Nuclide } & \multicolumn{2}{|c|}{ Uncertainty } \\
\cline { 2 - 3 } & "Clean MOX & MOX w/ Impurities \\
\hline pb210 & 1.0641 & 0.6387 \\
\hline ra226 & 1.2235 & 0.8533 \\
\hline ac227 & 0.6231 & 0.6575 \\
\hline th227 & 0.6231 & 0.6575 \\
\hline th230 & 1.1831 & 0.7945 \\
\hline pa231 & 0.6028 & 0.6460 \\
\hline u234 & 1.1110 & 0.7477 \\
\hline u235 & 1.3668 & 1.3539 \\
\hline u236 & 1.0367 & 1.0588 \\
\hline u237 & 1.9287 & 1.8627 \\
\hline u238 & 0.0918 & 0.0963 \\
\hline np237 & 0.7904 & 0.3727 \\
\hline np239 & 24.9776 & 23.0736 \\
\hline pu238 & 1.3121 & 0.7914 \\
\hline pu239 & 1.3840 & 1.3535 \\
\hline pu240 & 2.3612 & 2.2987 \\
\hline pu241 & 1.9287 & 1.8627 \\
\hline pu242 & 2.5902 & 2.3931 \\
\hline am241 & 1.8381 & 1.1829 \\
\hline am242m & 1.2130 & 1.1621 \\
\hline am243 & 24.9775 & 23.0737 \\
\hline cm242 & 1.2128 & 1.1618 \\
\hline cm244 & 17.6375 & 16.1298 \\
\hline cm245 & 14.4246 & 13.1267 \\
\hline c 14 & 0.8374 & 0.9462 \\
\hline se 79 & 0.7336 & 0.8164 \\
\hline sr 90 & 0.6899 & 0.7637 \\
\hline tc 99 & 2.5609 & 2.9419 \\
\hline i129 & 0.7641 & 0.8277 \\
\hline cs137 & 0.7591 & 0.8189 \\
\hline ba137m & 0.7591 & 0.8189 \\
\hline y 90 & 0.6899 & 0.7637 \\
\hline cs134 & 1.6727 & 1.6825 \\
\hline
\end{tabular}

Table 3.15: Isotopic uncertainties for MOX fuels.

\subsubsection{Comparison of Results, Simplified ORIGEN Models}

In the following pages, the two most commonly examined metrics for repository

performance and reprocessing are graphically compared: decay heat and radioactivity, spanning 10 to 10,000 years of decay time. These data are depicted as plots of the isotopic 
uncertainty information from the simplified ORIGEN models as propagated to these two key metrics. For additional information about each model, please refer to the previous sections. Attention is again drawn to the observation that, by experience, it is shown that major changes in the fuel composition, e.g. from UOX to MOX, cause changes to the distribution of isotopic uncertainties. Comparing the PWR UOX and PWR MOX fuels, which have the same geometry and are burned in the same reactor type, exemplifies this by showing a drop in uncertainties for plutonium isotopes, increase in americium and curium isotopic uncertainties, and fission product uncertainties more than doubling. This is quite dissimilar from when the BWR (in the 50-65\% void region) and PWR results are compared. Even with two different reactor types and two different geometries, those results are similar due to their fuel type and burnup.

Following the order in which the models were presented, the first comparison made is of the ORIGEN model representing a PWR, using both the stochastic method and the ESM approach for the same model. The nominal information is the same for each since it is the same model, only analyzed with different uncertainty analysis methods. Both the decay heat uncertainty (Figure 3.1) and the radioactivity uncertainty (Figure 3.2) were nearly identical between the two models, showing graphically that ESM yields the same results as forward perturbation.

Next, comparison is made between the metrics for the PWR model using the SAS2H updated flux spectrum and cross sections versus using the typical LWR spectrum and cross sections provided with the SCALE package for decay heat (Figure 3.3) and for radioactivity (Figure 3.4). While nominal decay performance was nearly the same, indicating similar 
isotopics results, the typical values tended to over-estimate decay heat uncertainty and underestimate radioactivity uncertainty after the first one hundred years.

The BWR models at various void fractions are compared next. In terms of nominal values, the different voids produced nearly the same short term decay heat (Figure 3.5) and radioactivity (Figure 3.6), but the variance can be seen in the later decay times. This variance in longer term performance is due to slightly different actinide buildup, which can be seen in the discharge isotopics presented in Section 3.1.4. The uncertainties which are shown to vary between different voids models do so due to a different flux spectrum used to collapse the covariance matrix with each different void. It is also observed that the magnitude of the relative uncertainties for the BWR is similar to that of the PWR, which uses the same UOX fuel. One notices a trend in the UOX fuels, that as time increases, the uncertainty tends to increase, typically around $100-500$ years. This is due to low uncertainty, high contributing fission products decaying away, leaving higher uncertainty, long lived actinides to decay.

Finally, the PWR model with UOX fuel is compared to the two PWR models using MOX fuels - both clean and with impurities - that were examined in this study. MOX fuels maintain higher heat load (Figure 3.7) and radioactivity (Figure 3.8) for a longer span of time than the PWR fuel due to the build up of long-lived actinides in the MOX fuel. Also, the MOX uncertainty is higher than that of the PWR UOX fuel due to the presence of higher quantities of actinides initially, longer burnup, and different operating conditions. 


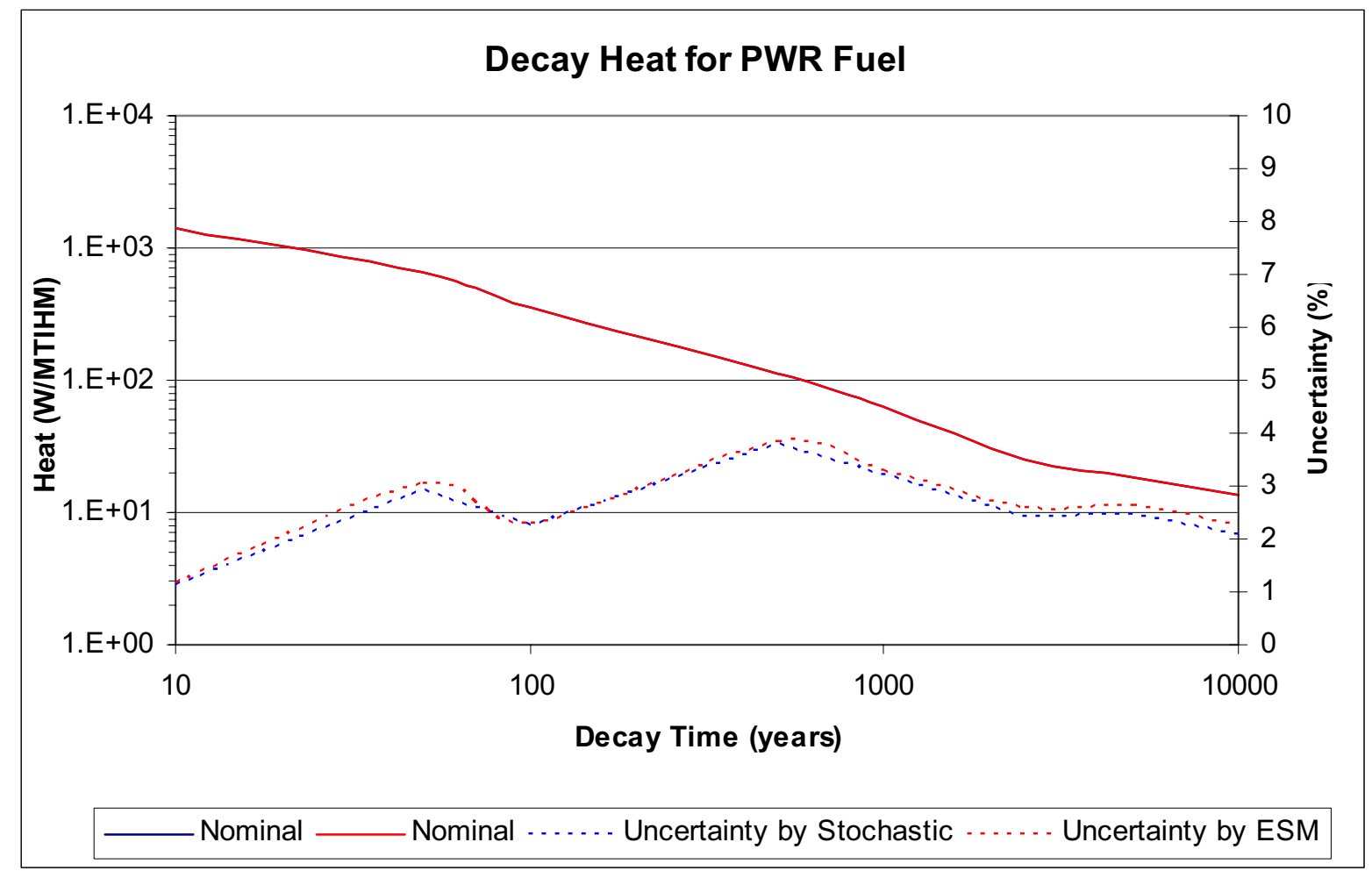

Figure 3.1: Decay heat comparison of stochastic and ESM sampling methods.

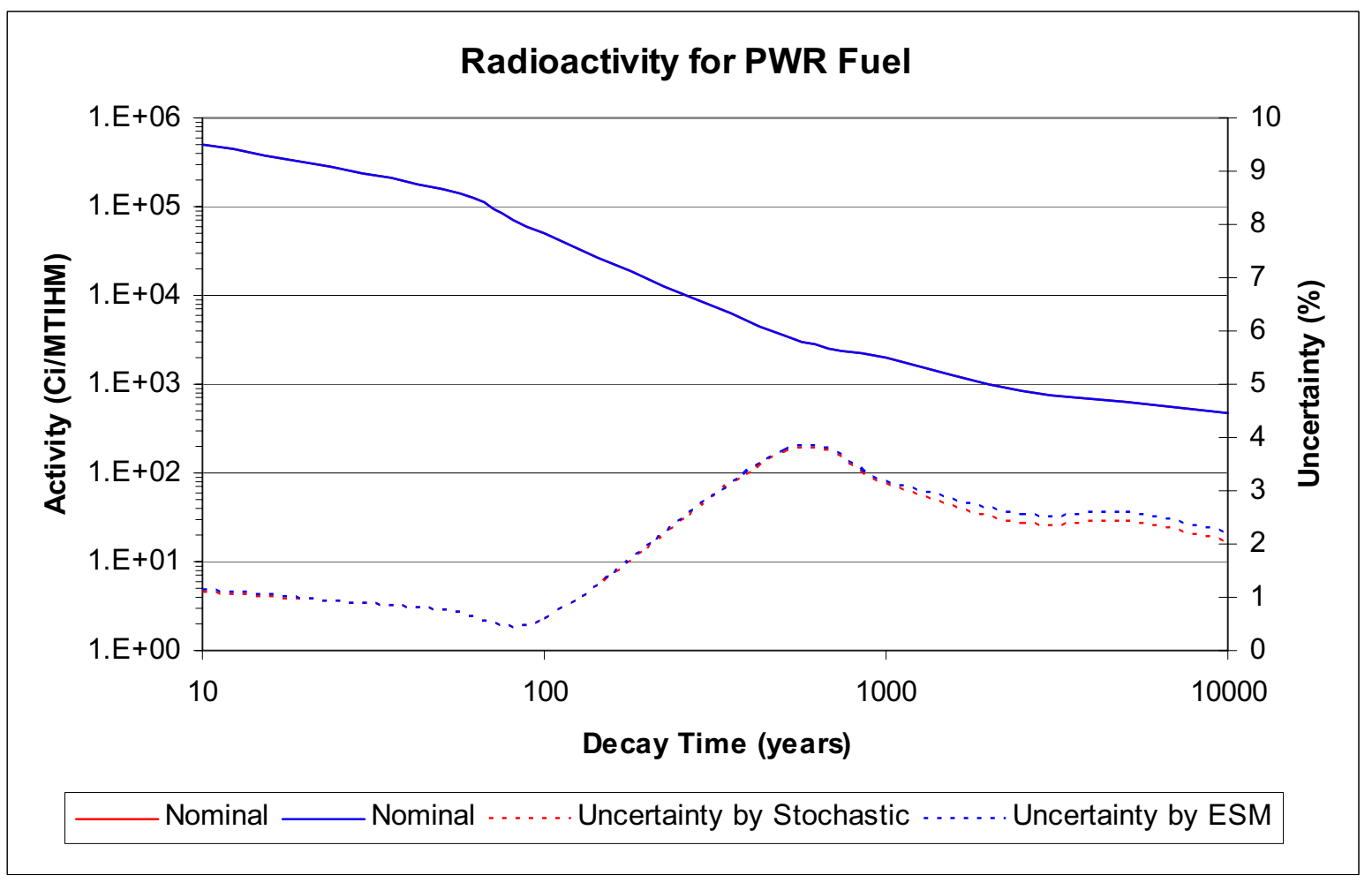

Figure 3.2: Radioactivity comparison of stochastic and ESM sampling methods. 


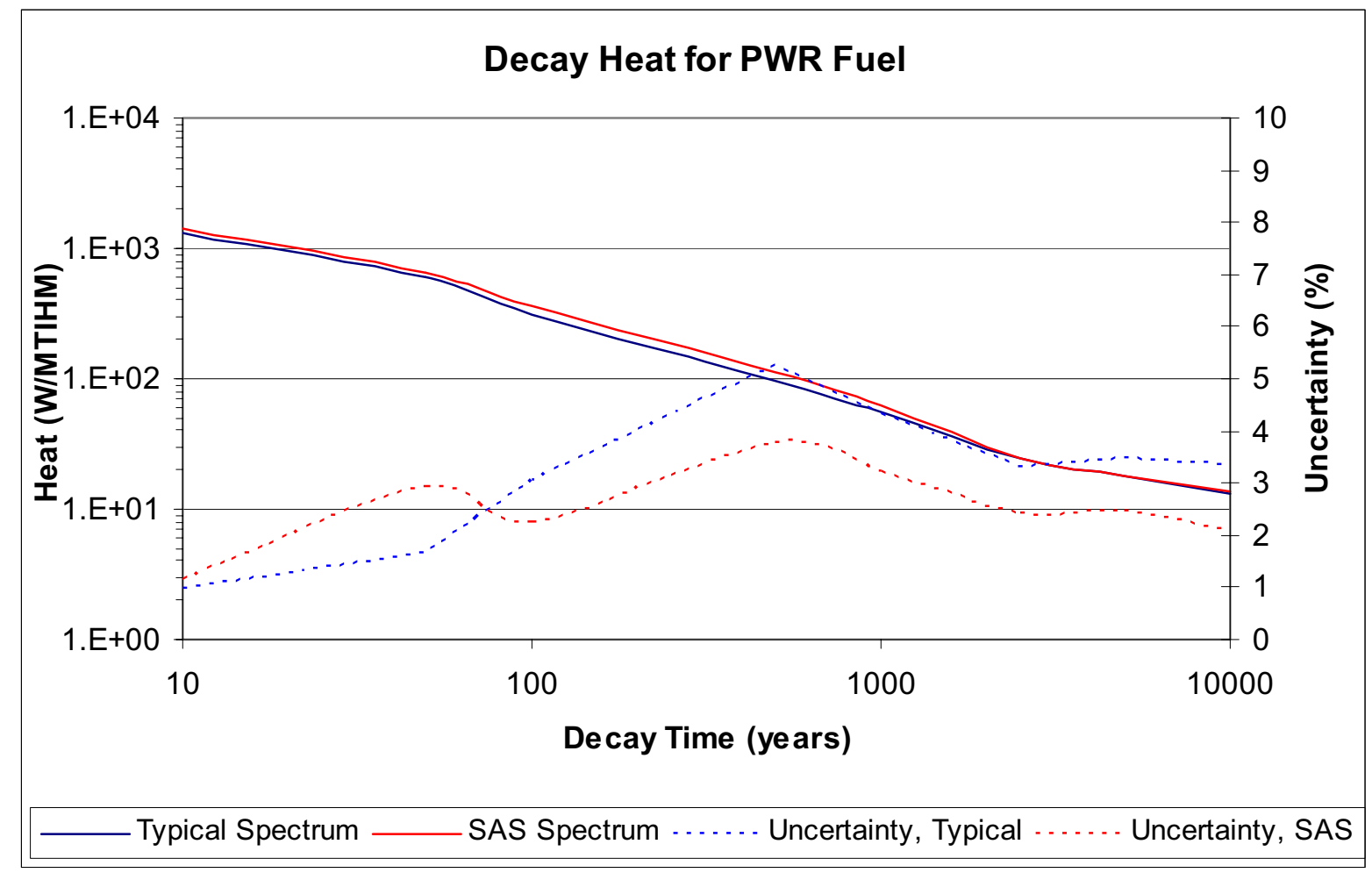

Figure 3.3: Decay heat comparison of SCALE provided data and SAS2H updated data.

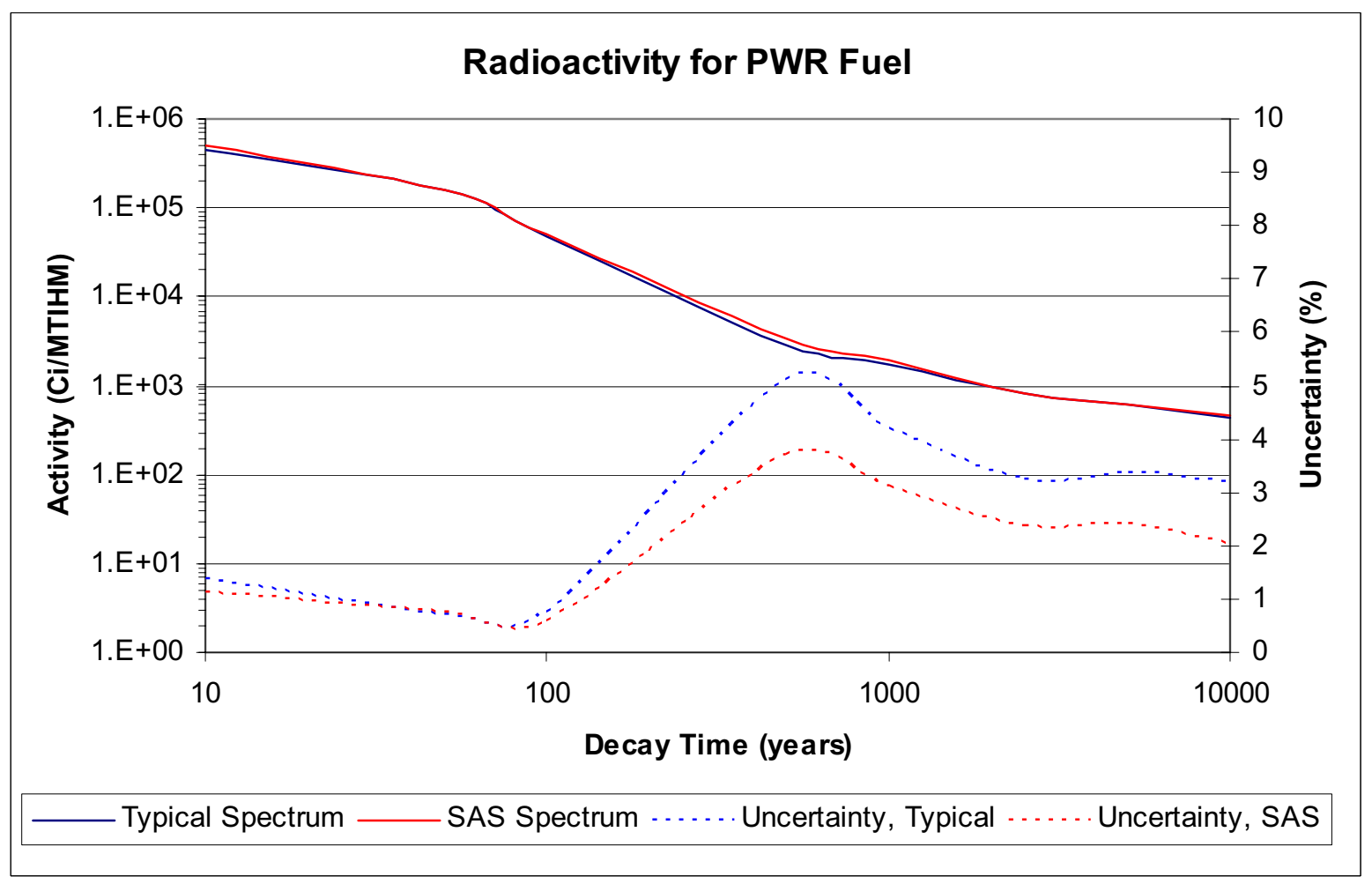

Figure 3.4: Radioactivity, SCALE provided data and SAS2H updated data. 


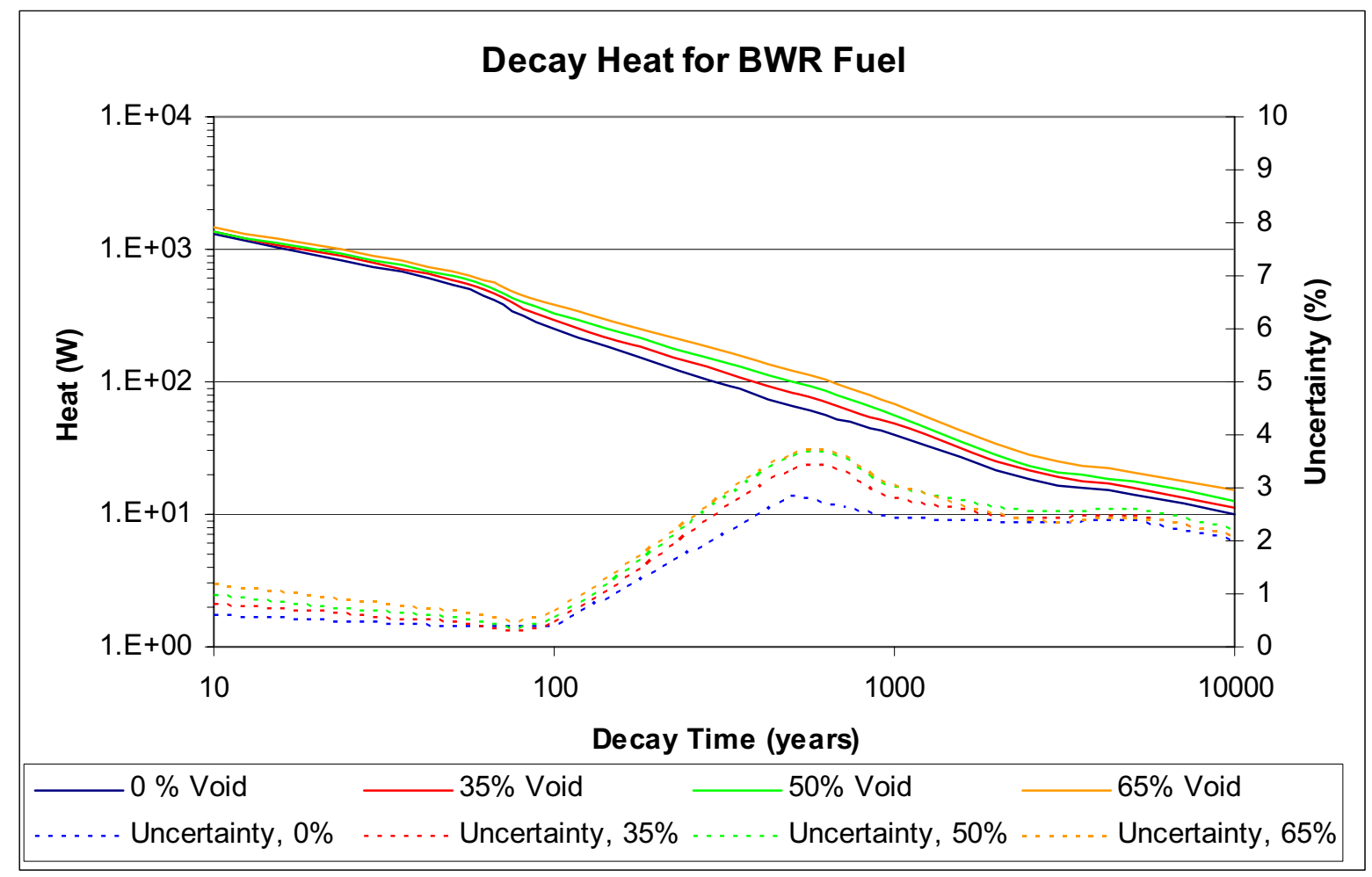

Figure 3.5: Decay heat comparison for BWR fuels.

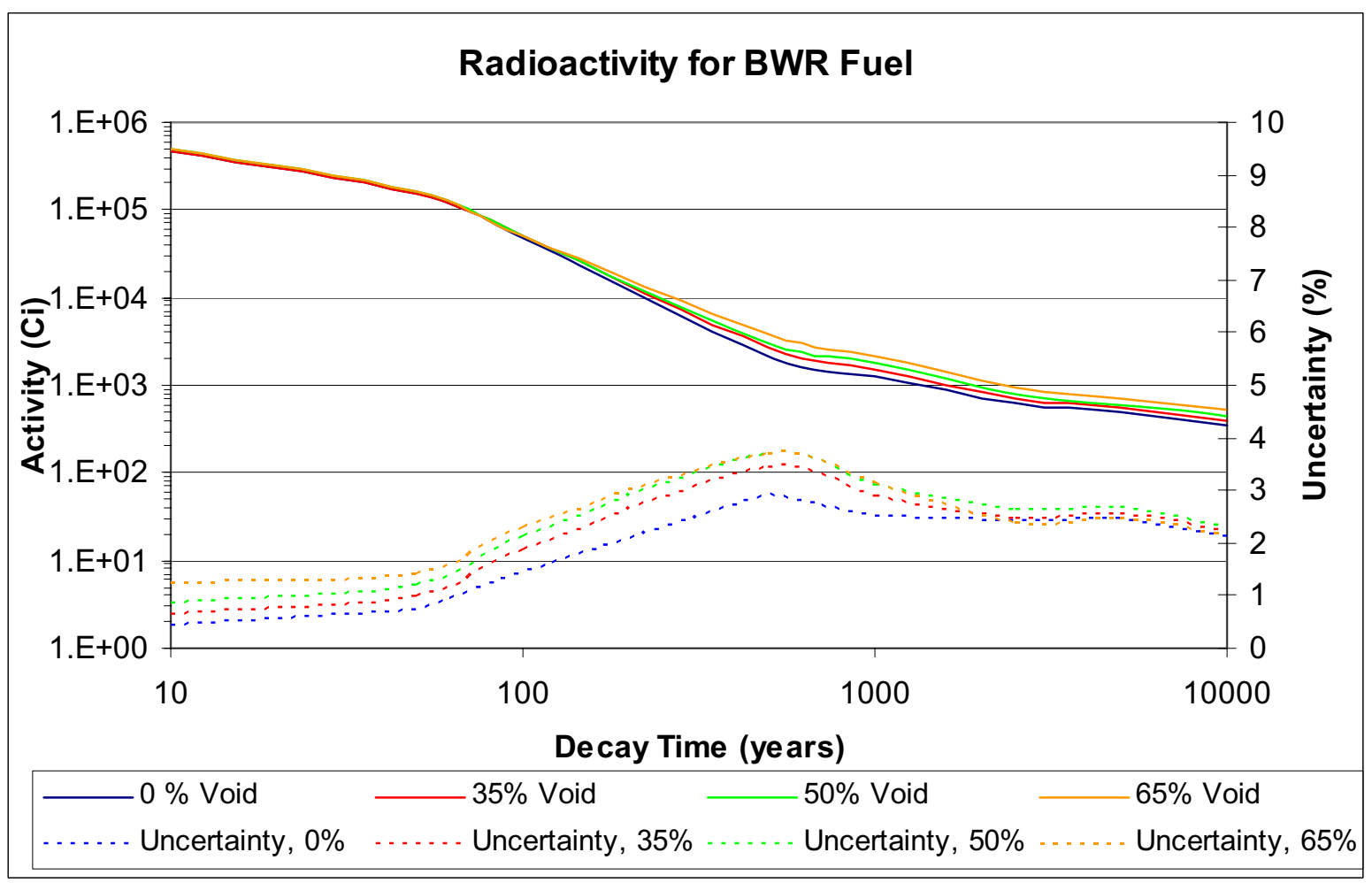

Figure 3.6: Radioactivity Comparison for BWR fuels. 


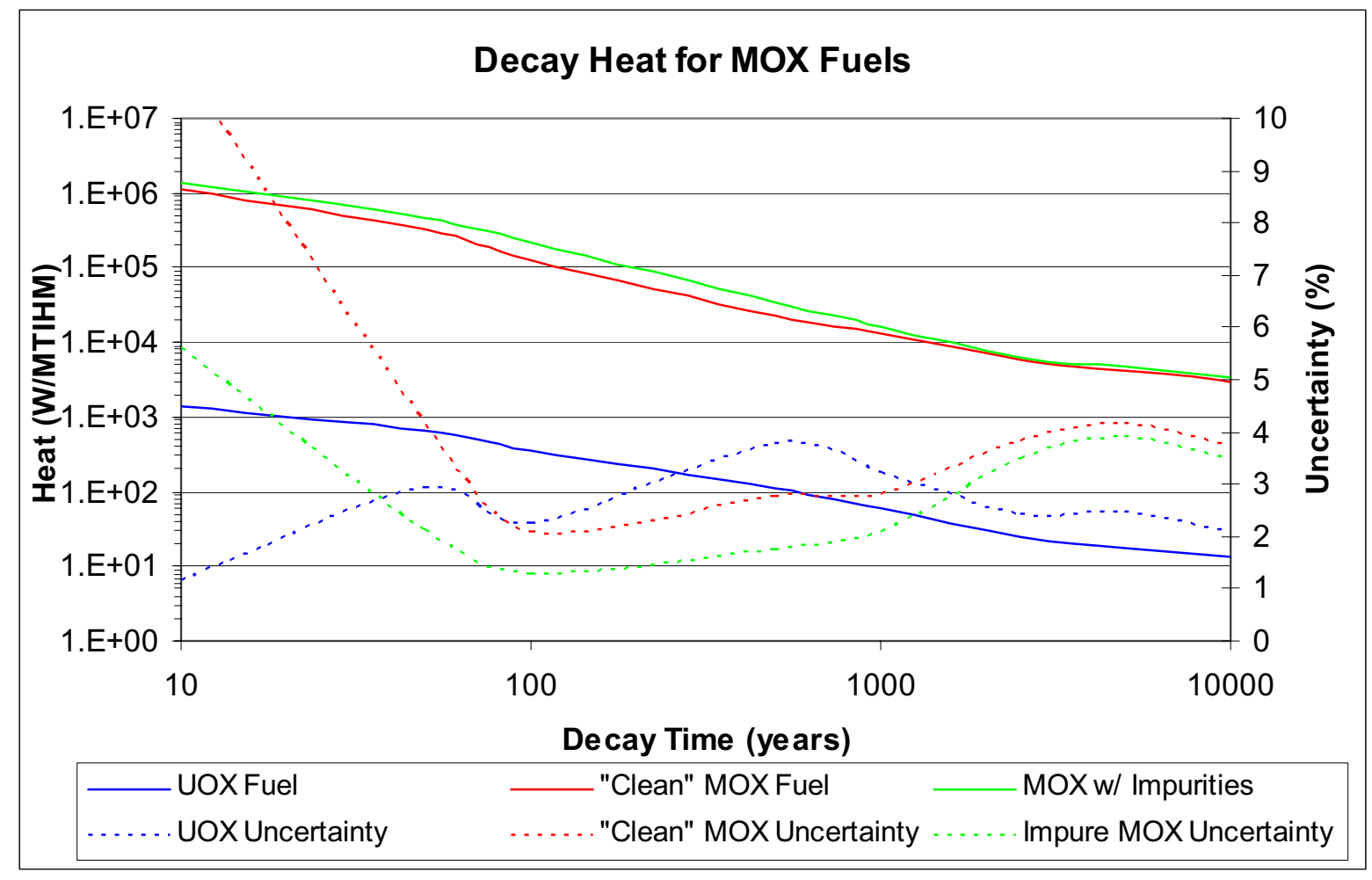

Figure 3.7: Decay heat comparison of UOX and MOX fuels.

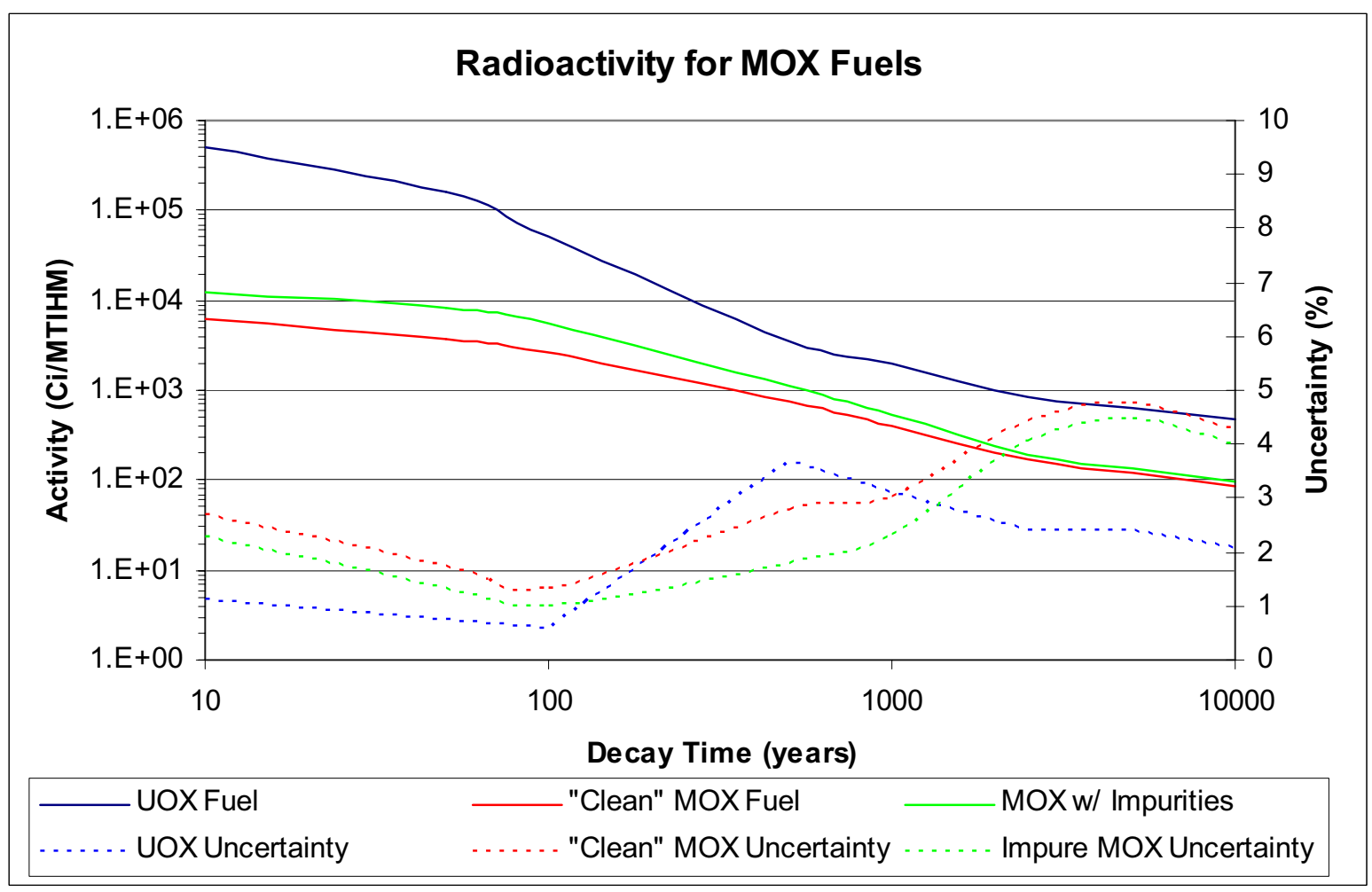

Figure 3.8: Radioactivity comparison for UOX and MOX fuels. 


\subsubsection{A Brief Experiment with Operational Uncertainties}

Since cross section uncertainties have shown almost negligible affects on the metrics of interest that have been examined in this study, whether operational parameters could outweigh these small cross section induced uncertainties was examined. A simple experiment was performed to test this hypothesis. The simplified, unperturbed PWR model in ORIGEN as discussed in Section 3.1.2 was depleted adjusting the following parameters: +/- 2 GWD/MTU burnup (a reasonable measurement uncertainty, [33]), and varied power history between 90 and $105 \%$ of full power over the life of the fuel. Data are presented for several key isotopes that are primary contributors to decay heat (Figure 3.9) and radioactivity (Figure 3.10), taken at a time of one hundred years after decay. In examining the following figures, operational uncertainties can clearly be seen to have a much greater impact on the metrics of interest than cross-section uncertainty.

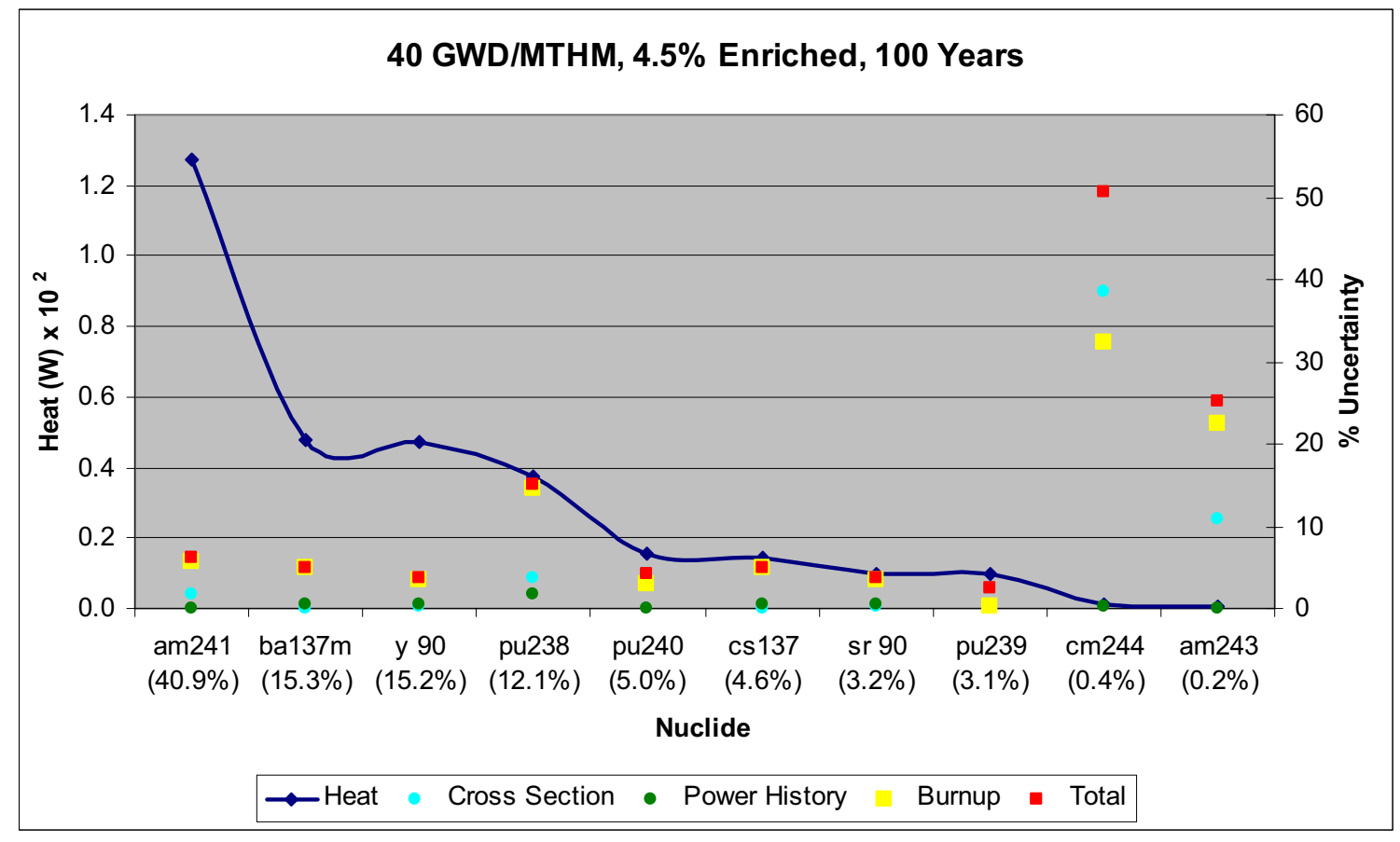

Figure 3.9: Decay heat uncertainty of key isotopes due to various sources. 


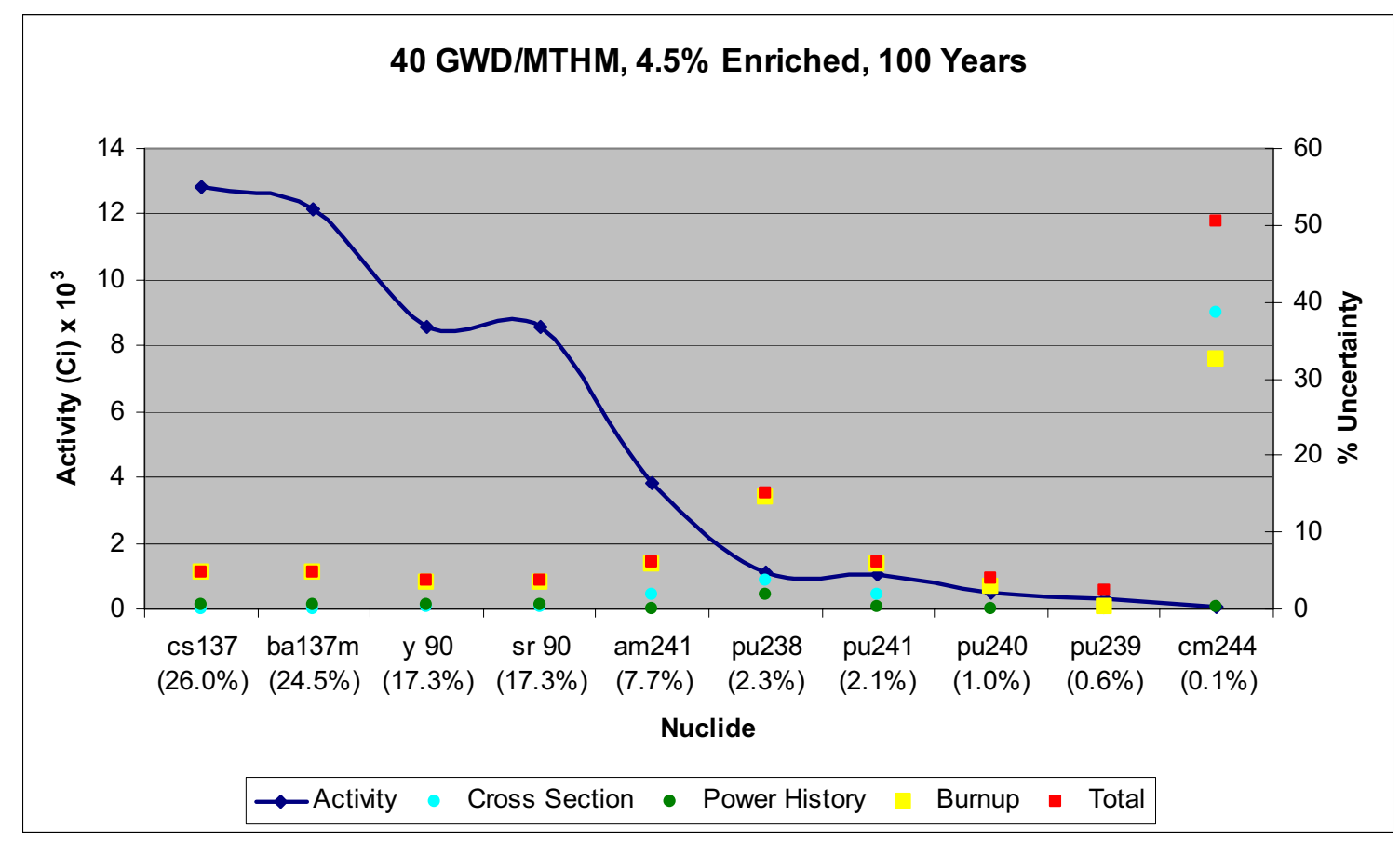

Figure 3.10: Radioactivity uncertainty in key isotopes due to various sources. 


\subsection{TRITON Models}

\subsubsection{PWR Model in TRITON with UOX Fuel}

The choice to move the analysis to using TRITON for the remaining fuel types has been discussed previously. The PWR model was reconstructed in the more rigorous TRITON sequence to 1) validate the uncertainty propagation approach of using ESM in TRITON, 2) compare to the SAS2H/ORIGEN model, 3) use a more detailed and finer model which is a procedure closer to standard fuel analysis, and 4) provide an isotopic covariance matrix for the spent PWR fuel that will be used in the recycling experiment. If TRITON, using only the Wigner cell, is given the same geometry, isotopics, and power history -- using one burnup step -- as SAS2H it will produce equivalent results (Table 3.16). Results are not identical due to different transport solutions, but the differences are statistically insignificant. Note that if more burnup steps are used or the buffer region equivalent to water holes is neglected in TRITON the results are no longer equivalent (Table 3.16). The model, using 4.5 w/o UOX fuel, was first burned to $40 \mathrm{GWD} / \mathrm{MTU}$ to match the SAS2H model, and then the burnup was extended to $48 \mathrm{GWD} / \mathrm{MTU}$ so as to provide a more realistic end of life keffective value. The 40 GWD model used 26 burnup steps while the 48 GWD model had 30 . The discharge isotopics of the 48 GWD/MTU model are given in Table 3.18, and it is these values to which uncertainty is propagated in later discussion and comparison. For completeness, the $40 \mathrm{GWD} / \mathrm{MTU}$ discharge isotopics are presented in Table 3.17 and the reader can see how an additional $8 \mathrm{GWD} / \mathrm{MTU}$ changes the fuel composition. 


\begin{tabular}{|c|c|c|c|c|}
\hline \multirow[b]{2}{*}{ Nuclide } & \multicolumn{4}{|c|}{ Number Density at 10 Years Decay, (g) } \\
\hline & $\begin{array}{c}\text { ORIGEN with } 1 \\
\text { SAS2H } \\
\text { Updated BU Step }\end{array}$ & $\begin{array}{l}\text { TRITON } \\
\text { with } \\
1 \text { BU Step }\end{array}$ & $\begin{array}{c}\text { TRITON with } 1 \mathrm{BU} \\
\text { Step without } \\
\text { Buffer }\end{array}$ & $\begin{array}{c}\begin{array}{c}\text { TRITON } \\
\text { with }\end{array} \\
4 \text { BU Steps }\end{array}$ \\
\hline pb210 & 5.054E-10 & $5.369 \mathrm{E}-10$ & $5.193 \mathrm{E}-10$ & 5.357E-10 \\
\hline ra226 & 3.165E-07 & 3.369E-07 & 3.239E-07 & 3.353E-07 \\
\hline ac227 & 6.941E-08 & 7.115E-08 & 7.796E-08 & 7.326E-08 \\
\hline th227 & 1.613E-10 & 1.653E-10 & $1.812 \mathrm{E}-10$ & $1.702 \mathrm{E}-10$ \\
\hline th230 & $5.585 \mathrm{E}-03$ & $5.951 \mathrm{E}-03$ & 5.766E-03 & $5.921 \mathrm{E}-03$ \\
\hline pa231 & 4.323E-04 & 4.412E-04 & 4.806E-04 & 4.537E-04 \\
\hline u234 & $1.675 \mathrm{E}+02$ & $1.781 \mathrm{E}+02$ & $1.754 \mathrm{E}+02$ & $1.771 \mathrm{E}+02$ \\
\hline $\mathrm{u} 235$ & $1.234 \mathrm{E}+04$ & $1.252 \mathrm{E}+04$ & $1.315 \mathrm{E}+04$ & $1.252 \mathrm{E}+04$ \\
\hline u236 & $5.526 \mathrm{E}+03$ & $5.526 \mathrm{E}+03$ & $5.570 \mathrm{E}+03$ & $5.555 \mathrm{E}+03$ \\
\hline u237 & $2.945 \mathrm{E}-05$ & 2.978E-05 & 3.463E-05 & 2.603E-05 \\
\hline $\mathrm{u} 238$ & $9.298 \mathrm{E}+05$ & $9.293 E+05$ & $9.265 \mathrm{E}+05$ & $9.292 \mathrm{E}+05$ \\
\hline np237 & $5.730 \mathrm{E}+02$ & $5.668 \mathrm{E}+02$ & $6.277 \mathrm{E}+02$ & $5.489 \mathrm{E}+02$ \\
\hline np239 & $1.001 \mathrm{E}-04$ & 1.012E-04 & 1.187E-04 & 9.312E-05 \\
\hline pu238 & $1.796 \mathrm{E}+02$ & $1.771 \mathrm{E}+02$ & $2.088 \mathrm{E}+02$ & $1.725 \mathrm{E}+02$ \\
\hline pu239 & $5.555 \mathrm{E}+03$ & $5.619 \mathrm{E}+03$ & $6.484 \mathrm{E}+03$ & $5.796 \mathrm{E}+03$ \\
\hline pu240 & $1.895 \mathrm{E}+03$ & $1.899 \mathrm{E}+03$ & $2.002 \mathrm{E}+03$ & $2.278 \mathrm{E}+03$ \\
\hline pu241 & $9.717 \mathrm{E}+02$ & $9.824 \mathrm{E}+02$ & $1.143 \mathrm{E}+03$ & $8.589 \mathrm{E}+02$ \\
\hline pu242 & $5.576 \mathrm{E}+02$ & $5.571 \mathrm{E}+02$ & $5.976 \mathrm{E}+02$ & $5.123 \mathrm{E}+02$ \\
\hline am241 & $6.407 \mathrm{E}+02$ & $6.533 E+02$ & $7.596 \mathrm{E}+02$ & $5.690 \mathrm{E}+02$ \\
\hline$a m 242 m$ & $8.756 \mathrm{E}-01$ & 9.965E-01 & $1.237 \mathrm{E}+00$ & 7.995E-01 \\
\hline am243 & $1.163 E+02$ & 1.176E+02 & $1.380 \mathrm{E}+02$ & $1.082 \mathrm{E}+02$ \\
\hline $\mathrm{cm} 242$ & $2.284 \mathrm{E}-03$ & 2.599E-03 & 3.226E-03 & $2.086 \mathrm{E}-03$ \\
\hline $\mathrm{cm} 244$ & $2.200 \mathrm{E}+01$ & $2.204 \mathrm{E}+01$ & $2.826 \mathrm{E}+01$ & $2.087 \mathrm{E}+01$ \\
\hline $\mathrm{cm} 245$ & $1.207 E+00$ & $1.218 \mathrm{E}+00$ & $1.773 \mathrm{E}+00$ & $1.213 \mathrm{E}+00$ \\
\hline c 14 & $3.331 \mathrm{E}-03$ & 3.327E-03 & $3.461 \mathrm{E}-03$ & 3.324E-03 \\
\hline se 79 & $5.817 \mathrm{E}+00$ & $5.804 \mathrm{E}+00$ & $5.878 \mathrm{E}+00$ & $5.796 \mathrm{E}+00$ \\
\hline sr 90 & $5.393 E+02$ & $5.363 E+02$ & $5.342 \mathrm{E}+02$ & $5.366 \mathrm{E}+02$ \\
\hline tc 99 & $9.622 \mathrm{E}+02$ & $9.603 E+02$ & $9.705 \mathrm{E}+02$ & $9.585 \mathrm{E}+02$ \\
\hline i129 & $1.760 \mathrm{E}+02$ & $1.762 E+02$ & $1.839 \mathrm{E}+02$ & $1.755 \mathrm{E}+02$ \\
\hline cs137 & $1.179 \mathrm{E}+03$ & $1.175 E+03$ & $1.199 \mathrm{E}+03$ & $1.173 E+03$ \\
\hline ba137m & $1.801 \mathrm{E}-04$ & 1.794E-04 & $1.831 \mathrm{E}-04$ & 1.791E-04 \\
\hline y90 & $1.401 \mathrm{E}-01$ & 1.394E-01 & 1.388E-01 & 1.394E-01 \\
\hline cs134 & $4.990 \mathrm{E}+00$ & $4.749 \mathrm{E}+00$ & $5.162 \mathrm{E}+00$ & $4.751 \mathrm{E}+00$ \\
\hline
\end{tabular}

Table 3.16: Comparison of isotopics between models. 


\begin{tabular}{|l|l|l|l|l|l|}
\hline \multicolumn{7}{|c|}{ Discharge Isotopics, grams / MTHM } \\
\hline pb210 & $3.068 \mathrm{E}-11$ & np237 & $5.327 \mathrm{E}+02$ & cm244 & $2.989 \mathrm{E}+01$ \\
\hline ra226 & $2.051 \mathrm{E}-08$ & np239 & $8.249 \mathrm{E}+01$ & cm245 & $1.188 \mathrm{E}+00$ \\
\hline ac227 & $4.409 \mathrm{E}-09$ & pu238 & $1.743 \mathrm{E}+02$ & c 14 & $3.337 \mathrm{E}-03$ \\
\hline th227 & $1.286 \mathrm{E}-11$ & pu239 & $5.745 \mathrm{E}+03$ & se 79 & $5.807 \mathrm{E}+00$ \\
\hline th230 & $1.311 \mathrm{E}-03$ & pu240 & $2.342 \mathrm{E}+03$ & sr 90 & $6.853 \mathrm{E}+02$ \\
\hline pa231 & $3.637 \mathrm{E}-04$ & pu241 & $1.362 \mathrm{E}+03$ & tc 99 & $9.752 \mathrm{E}+02$ \\
\hline $\mathbf{u 2 3 4}$ & $1.630 \mathrm{E}+02$ & pu242 & $5.071 \mathrm{E}+02$ & i129 & $1.741 \mathrm{E}+02$ \\
\hline $\mathbf{u 2 3 5}$ & $1.248 \mathrm{E}+04$ & am241 & $4.344 \mathrm{E}+01$ & cs137 & $1.476 \mathrm{E}+03$ \\
\hline $\mathbf{u 2 3 6}$ & $5.563 \mathrm{E}+03$ & am242m & $8.884 \mathrm{E}-01$ & ba137m & $2.268 \mathrm{E}-04$ \\
\hline $\mathbf{u 2 3 7}$ & $1.108 \mathrm{E}+01$ & am243 & $1.062 \mathrm{E}+02$ & y90 & $1.844 \mathrm{E}-01$ \\
\hline $\mathbf{u 2 3 8}$ & $9.291 \mathrm{E}+05$ & $\mathbf{c m 2 4 2}$ & $1.277 \mathrm{E}+01$ & cs134 & $1.346 \mathrm{E}+02$ \\
\hline
\end{tabular}

Table 3.17: 40 GWD/MTU Discharge Isotopics

\begin{tabular}{|l|l|l|l|l|l|}
\hline \multicolumn{7}{|c|}{ Discharge Isotopics, grams / MTHM } \\
\hline pb210 & $5.772 \mathrm{E}-11$ & np237 & $6.646 \mathrm{E}+02$ & cm244 & $6.780 \mathrm{E}+01$ \\
\hline ra226 & $2.667 \mathrm{E}-08$ & np239 & $8.826 \mathrm{E}+01$ & cm245 & $3.088 \mathrm{E}+00$ \\
\hline ac227 & $5.433 \mathrm{E}-09$ & pu238 & $2.678 \mathrm{E}+02$ & c 14 & $4.127 \mathrm{E}-03$ \\
\hline th227 & $1.827 \mathrm{E}-11$ & pu239 & $5.746 \mathrm{E}+03$ & se 79 & $6.827 \mathrm{E}+00$ \\
\hline th230 & $1.370 \mathrm{E}-03$ & pu240 & $2.716 \mathrm{E}+03$ & sr 90 & $7.807 \mathrm{E}+02$ \\
\hline pa231 & $4.338 \mathrm{E}-04$ & pu241 & $1.566 \mathrm{E}+03$ & tc 99 & $1.135 \mathrm{E}+03$ \\
\hline u234 & $1.436 \mathrm{E}+02$ & pu242 & $7.621 \mathrm{E}+02$ & i129 & $2.141 \mathrm{E}+02$ \\
\hline u235 & $8.934 \mathrm{E}+03$ & am241 & $5.407 \mathrm{E}+01$ & cs137 & $1.757 \mathrm{E}+03$ \\
\hline $\mathbf{u 2 3 6}$ & $5.989 \mathrm{E}+03$ & am242m & $1.111 \mathrm{E}+00$ & ba137m & $2.700 \mathrm{E}-04$ \\
\hline $\mathbf{u 2 3 7}$ & $1.240 \mathrm{E}+01$ & am243 & $1.883 \mathrm{E}+02$ & y90 & $2.112 \mathrm{E}-01$ \\
\hline u238 & $9.228 \mathrm{E}+05$ & $\mathbf{c m 2 4 2}$ & $1.912 \mathrm{E}+01$ & cs134 & $1.824 \mathrm{E}+02$ \\
\hline
\end{tabular}

Table 3.18: 48 GWD/MTU Discharge Isotopics

The ESM approach was implemented in TRITON using the eigenvalues and

eigenvectors of the 44-group covariance matrix for selected reactions to perturb the 44-group master cross section library input to the code. A total of 1938 samples were run as this

number was the effective rank of this covariance matrix obtained by limiting perturbations to those corresponding to eigenvalues greater than $10^{-6}$ relative to the reference cross section.

Results for uncertainty of the 33 tracked nuclides are presented in Table 3.19, along with the uncertainty results from the simple ORIGEN 1-group model using the direct perturbation method. Appendix C present further details. 


\begin{tabular}{|l|c|c|}
\hline & \multicolumn{2}{|c|}{$\begin{array}{c}\text { \% Standard } \\
\text { Deviation }\end{array}$} \\
\hline Nuclide & TRITON & ORIGEN \\
\hline pb210 & $\mathbf{0 . 1 5 2}$ & $\mathbf{1 . 1 9 5}$ \\
\hline ra226 & $\mathbf{0 . 1 8 5}$ & $\mathbf{1 . 3 5 5}$ \\
\hline ac227 & $\mathbf{0 . 2 3 1}$ & $\mathbf{0 . 4 8 0}$ \\
\hline th227 & $\mathbf{0 . 2 3 3}$ & $\mathbf{0 . 4 8 0}$ \\
\hline th230 & $\mathbf{0 . 1 8 5}$ & $\mathbf{1 . 4 9 1}$ \\
\hline pa231 & $\mathbf{0 . 2 5 3}$ & $\mathbf{0 . 5 1 8}$ \\
\hline u234 & $\mathbf{0 . 1 7 3}$ & $\mathbf{1 . 5 7 9}$ \\
\hline u235 & $\mathbf{0 . 5 8 3}$ & $\mathbf{1 . 3 1 2}$ \\
\hline u236 & $\mathbf{1 . 0 4 8}$ & $\mathbf{0 . 7 1 2}$ \\
\hline u237 & $\mathbf{2 . 0 5 3}$ & $\mathbf{2 . 5 2 1}$ \\
\hline u238 & $\mathbf{0 . 0 1 5}$ & $\mathbf{0 . 0 7 8}$ \\
\hline np237 & $\mathbf{1 . 6 0 2}$ & $\mathbf{0 . 6 0 5}$ \\
\hline np239 & $\mathbf{3 . 9 1 0}$ & $\mathbf{1 3 . 6 4 3}$ \\
\hline pu238 & $\mathbf{1 . 7 5 1}$ & $\mathbf{1 . 0 5 0}$ \\
\hline pu239 & $\mathbf{1 . 0 4 5}$ & $\mathbf{0 . 8 0 6}$ \\
\hline pu240 & $\mathbf{2 . 4 9 0}$ & $\mathbf{2 . 5 6 6}$ \\
\hline pu241 & $\mathbf{2 . 0 5 3}$ & $\mathbf{2 . 5 2 1}$ \\
\hline pu242 & $\mathbf{3 . 9 0 7}$ & $\mathbf{2 . 5 9 8}$ \\
\hline am241 & $\mathbf{2 . 0 5 3}$ & $\mathbf{2 . 5 0 2}$ \\
\hline am242m & $\mathbf{2 . 1 0 4}$ & $\mathbf{2 . 1 7 2}$ \\
\hline am243 & $\mathbf{3 . 9 0 8}$ & $\mathbf{1 3 . 6 4 3}$ \\
\hline cm242 & $\mathbf{2 . 1 0 7}$ & $\mathbf{2 . 1 7 2}$ \\
\hline cm244 & $\mathbf{4 . 3 4 5}$ & $\mathbf{1 1 . 3 4 0}$ \\
\hline cm245 & $\mathbf{4 . 6 8 0}$ & $\mathbf{1 0 . 2 5 1}$ \\
\hline c 14 & $\mathbf{0 . 3 7 9}$ & $\mathbf{0 . 4 5 2}$ \\
\hline se 79 & $\mathbf{0 . 0 8 8}$ & $\mathbf{0 . 3 6 6}$ \\
\hline sr 90 & $\mathbf{0 . 1 1 7}$ & $\mathbf{0 . 3 7 5}$ \\
\hline tc 99 & $\mathbf{0 . 0 7 5}$ & $\mathbf{1 . 9 7 0}$ \\
\hline i129 & $\mathbf{0 . 2 4 1}$ & $\mathbf{0 . 4 1 9}$ \\
\hline cs137 & $\mathbf{0 . 0 3 2}$ & $\mathbf{0 . 3 8 2}$ \\
\hline ba137m & $\mathbf{0 . 0 3 2}$ & $\mathbf{0 . 3 8 2}$ \\
\hline y 90 & $\mathbf{0 . 1 0 7}$ & $\mathbf{0 . 3 7 5}$ \\
\hline cs134 & $\mathbf{0 . 3 0 0}$ & $\mathbf{1 . 1 3 8}$ \\
\hline
\end{tabular}

Table 3.19: Resulting standard deviations from both models.

At first glance, one is likely to say that the models are not equivalent. Rigorous and thorough search for the difference, however, yielded an explanation of these differences.

Both a primary and a secondary reason for the differences were discovered. Discussed first will be the secondary cause, as it has more tangible data. Consider carefully what terms of 
the uncertainty are being propagated in each model. Let the result, $\bar{y}$, of a perturbed model be defined as:

$$
\left.\bar{y}=\bar{\Omega} \mid{\overline{\sigma_{0}}}^{T}{\overline{\phi_{0}}}+\overline{\Delta \sigma}^{T} \bar{\phi}_{0}+{\overline{\sigma_{0}}}^{T} \overline{\Delta \phi}+\overline{\Delta \sigma}^{T} \overline{\Delta \phi}\right]
$$

where $\left\|\overline{\phi_{0}}+\overline{\Delta \phi}\right\|_{1}=1 .{\overline{\sigma_{0}}}^{T} \overline{\phi_{0}}$ is the cross sections collapsed using the BOL, unchanged flux, $\overline{\Delta \sigma}^{T} \bar{\phi}_{0}$ is the perturbation added by a cross section perturbations, ${\overline{\sigma_{0}}}^{T} \overline{\Delta \phi}$ is the perturbation added by an updated burnup dependent flux spectrum, and $\overline{\Delta \sigma}^{T} \overline{\Delta \phi}$ is the second order perturbation added by both sources, which is assumed to be negligible. A nominal run of ORIGEN inputs ${\overline{\sigma_{0}}}^{T} \overline{\phi_{0}}$. When making perturbations only in the ORIGEN input via the collapsed covariance data only the term $\overline{\Delta \sigma}^{T} \bar{\phi}_{0}$ is captured. When using a sequence such as TRITON that performs a transport update of flux and applies it to the master library, however, the term ${\overline{\sigma_{0}}}^{T} \overline{\Delta \phi}$ is also captured. Table 3.20 contains 44-group fluxes that have been normalized to the 1-group BOL flux, i.e. the sum of the BOL column of fluxes is equal to 1 . As can be seen in Table 3.20, as fuel depletes with burnup, the groups from $(0.1-3.0)$ ev tend to have the group fluxes decrease in value. Even though the flux is normalized by ORIGEN to keep the same power density, the overall shape of the flux changes. The energy spectra shift causes the term ${\overline{\sigma_{0}}}^{T} \overline{\Delta \phi}$ to be negative and reduces the overall uncertainty. A numerical experiment of this is shown in Table 3.21where the 44-group variance was collapsed using both averaged and burnup averaged flux changes from Table 3.20. Since the 1-group variances are collapsed from the 44-group using the flux, this directly affects the magnitude of the 1-group covariance values. More explicitly, the change in the 1-group covariance, $\overline{\overline{\Delta \operatorname{COV}(\sigma)}}$, due to a change in the 44 -group flux, $\overline{\Delta \phi}$, is: 


$$
\overline{\overline{\Delta \operatorname{COV}(\sigma)}}=\bar{\phi}_{0}^{T} \overline{\overline{\operatorname{COV}(\bar{\sigma})}} \overline{\Delta \phi}+\overline{\Delta \phi}^{T} \overline{\overline{\operatorname{COV}(\bar{\sigma})}} \overline{\phi_{0}}
$$

where $\left\|\overline{\phi_{0}}+\overline{\Delta \phi}\right\|_{1}=1$. As shown in Table 3.22, the reduced covariance data cause output uncertainties to be reduced. These affects can account for at most about $20 \%$ of the difference between the two models. 


\begin{tabular}{|c|c|c|c|c|c|}
\hline \multirow{2}{*}{$\begin{array}{l}\text { ENERGY } \\
\text { (ev) }\end{array}$} & \multirow{2}{*}{$\begin{array}{l}\text { BOL } \\
\text { FLUX }\end{array}$} & \multicolumn{4}{|c|}{ Flux at Various Burnups (MWD/MTU) } \\
\hline & & 2000 & 14000 & 26000 & 38000 \\
\hline $2.00 \mathrm{E}+07$ & 1.5651E-03 & 1.5763E-03 & 1.6394E-03 & 1.6954E-03 & 1.7454E-03 \\
\hline $8.19 E+06$ & $4.4291 \mathrm{E}-03$ & 4.4524E-03 & 4.5744E-03 & 4.6727E-03 & 4.7564E-03 \\
\hline $6.43 E+06$ & 1.3934E-02 & 1.3987E-02 & $1.4250 \mathrm{E}-02$ & 1.4445E-02 & 1.4606E-02 \\
\hline $4.80 \mathrm{E}+06$ & 4.6375E-02 & $4.6501 \mathrm{E}-02$ & 4.7090E-02 & 4.7490E-02 & 4.7804E-02 \\
\hline $3.00 \mathrm{E}+06$ & 3.0549E-02 & 3.0614E-02 & 3.0917E-02 & 3.1119E-02 & 3.1279E-02 \\
\hline $2.48 E+06$ & $9.2170 \mathrm{E}-03$ & 9.2356E-03 & 9.3223E-03 & $9.3810 \mathrm{E}-03$ & 9.4283E-03 \\
\hline $2.35 E+06$ & 3.7375E-02 & 3.7434E-02 & 3.7707E-02 & 3.7891E-02 & 3.8038E-02 \\
\hline $1.85 E+06$ & $4.6158 \mathrm{E}-02$ & $4.6211 \mathrm{E}-02$ & 4.6462E-02 & 4.6639E-02 & $4.6788 \mathrm{E}-02$ \\
\hline $1.40 \mathrm{E}+06$ & $6.4489 \mathrm{E}-02$ & $6.4525 \mathrm{E}-02$ & $6.4697 \mathrm{E}-02$ & $6.4832 \mathrm{E}-02$ & $6.4951 \mathrm{E}-02$ \\
\hline $9.00 \mathrm{E}+05$ & 1.1652E-01 & 1.1653E-01 & 1.1664E-01 & 1.1676E-01 & 1.1687E-01 \\
\hline $4.00 E+05$ & 1.1869E-01 & 1.1872E-01 & 1.1885E-01 & 1.1900E-01 & 1.1915E-01 \\
\hline $1.00 \mathrm{E}+05$ & $6.7525 \mathrm{E}-02$ & $6.7549 \mathrm{E}-02$ & $6.7674 \mathrm{E}-02$ & $6.7801 \mathrm{E}-02$ & 6.7913E-02 \\
\hline $2.50 \mathrm{E}+04$ & $1.5345 \mathrm{E}-02$ & $1.5351 \mathrm{E}-02$ & $1.5386 \mathrm{E}-02$ & $1.5420 \mathrm{E}-02$ & $1.5450 \mathrm{E}-02$ \\
\hline $1.70 \mathrm{E}+04$ & 6.3885E-02 & $6.3925 \mathrm{E}-02$ & $6.4125 \mathrm{E}-02$ & $6.4312 \mathrm{E}-02$ & $6.4473 \mathrm{E}-02$ \\
\hline $3.00 \mathrm{E}+03$ & 5.7312E-02 & 5.7371E-02 & 5.7669E-02 & 5.7936E-02 & $5.8161 \mathrm{E}-02$ \\
\hline $5.50 \mathrm{E}+02$ & $5.3043 \mathrm{E}-02$ & $5.3131 \mathrm{E}-02$ & $5.3575 \mathrm{E}-02$ & 5.3974E-02 & $5.4312 \mathrm{E}-02$ \\
\hline $1.00 \mathrm{E}+02$ & 3.3399E-02 & 3.3474E-02 & 3.3846E-02 & 3.4203E-02 & $3.4521 \mathrm{E}-02$ \\
\hline $3.00 \mathrm{E}+01$ & $2.7654 \mathrm{E}-02$ & $2.7687 \mathrm{E}-02$ & $2.7868 \mathrm{E}-02$ & $2.8123 \mathrm{E}-02$ & $2.8389 \mathrm{E}-02$ \\
\hline $1.00 \mathrm{E}+01$ & 5.0899E-03 & $5.1134 \mathrm{E}-03$ & $5.2116 \mathrm{E}-03$ & $5.2966 \mathrm{E}-03$ & $5.3718 \mathrm{E}-03$ \\
\hline $8.10 E+00$ & $5.0429 \mathrm{E}-03$ & $5.0502 \mathrm{E}-03$ & $5.0835 \mathrm{E}-03$ & $5.1301 \mathrm{E}-03$ & 5.1791E-03 \\
\hline $6.00 \mathrm{E}+00$ & $5.2123 \mathrm{E}-03$ & $5.1352 \mathrm{E}-03$ & $4.7772 \mathrm{E}-03$ & $4.5935 \mathrm{E}-03$ & 4.4920E-03 \\
\hline $4.75 \mathrm{E}+00$ & 1.1050E-02 & 1.1047E-02 & 1.1012E-02 & 1.1009E-02 & 1.1022E-02 \\
\hline $3.00 \mathrm{E}+00$ & 1.3506E-02 & $1.3511 \mathrm{E}-02$ & 1.3504E-02 & 1.3459E-02 & 1.3353E-02 \\
\hline $1.77 E+00$ & 1.4724E-02 & 1.4636E-02 & 1.3081E-02 & 1.2225E-02 & 1.1719E-02 \\
\hline $1.00 \mathrm{E}+00$ & 1.2651E-02 & $1.2620 \mathrm{E}-02$ & 1.1948E-02 & 1.1464E-02 & 1.1130E-02 \\
\hline $6.25 \mathrm{E}-01$ & $1.2905 \mathrm{E}-02$ & 1.2795E-02 & 1.2113E-02 & 1.1786E-02 & 1.1611E-02 \\
\hline 4.00E-01 & 1.9535E-03 & $1.9051 \mathrm{E}-03$ & 1.7194E-03 & 1.6557E-03 & 1.6395E-03 \\
\hline $3.75 \mathrm{E}-01$ & $2.1397 \mathrm{E}-03$ & $2.0608 \mathrm{E}-03$ & $1.7892 \mathrm{E}-03$ & $1.7019 \mathrm{E}-03$ & $1.6831 \mathrm{E}-03$ \\
\hline $3.50 \mathrm{E}-01$ & $2.3695 \mathrm{E}-03$ & $2.2414 \mathrm{E}-03$ & $1.8389 \mathrm{E}-03$ & 1.7169E-03 & 1.6920E-03 \\
\hline $3.25 \mathrm{E}-01$ & $5.7177 \mathrm{E}-03$ & $5.2816 \mathrm{E}-03$ & $4.0465 \mathrm{E}-03$ & $3.6950 \mathrm{E}-03$ & $3.6285 \mathrm{E}-03$ \\
\hline $2.75 \mathrm{E}-01$ & 3.6375E-03 & $3.3722 \mathrm{E}-03$ & $2.6589 \mathrm{E}-03$ & $2.4553 \mathrm{E}-03$ & $2.4277 \mathrm{E}-03$ \\
\hline $2.50 \mathrm{E}-01$ & 4.4406E-03 & $4.1571 \mathrm{E}-03$ & 3.4519E-03 & 3.2579E-03 & 3.2562E-03 \\
\hline $2.25 \mathrm{E}-01$ & $5.4824 \mathrm{E}-03$ & $5.1577 \mathrm{E}-03$ & $4.4577 \mathrm{E}-03$ & $4.2966 \mathrm{E}-03$ & $4.3487 \mathrm{E}-03$ \\
\hline $2.00 \mathrm{E}-01$ & 1.5222E-02 & $1.4320 \mathrm{E}-02$ & 1.2882E-02 & 1.2742E-02 & 1.3134E-02 \\
\hline $1.50 \mathrm{E}-01$ & $2.2839 \mathrm{E}-02$ & $2.1257 \mathrm{E}-02$ & 1.9756E-02 & $2.0051 \mathrm{E}-02$ & $2.1107 \mathrm{E}-02$ \\
\hline $1.00 \mathrm{E}-01$ & 1.7676E-02 & $1.6305 \mathrm{E}-02$ & 1.5454E-02 & 1.5962E-02 & $1.7058 \mathrm{E}-02$ \\
\hline $7.00 \mathrm{E}-02$ & 1.2312E-02 & 1.1381E-02 & 1.0939E-02 & 1.1434E-02 & 1.2344E-02 \\
\hline $5.00 \mathrm{E}-02$ & $5.7830 \mathrm{E}-03$ & $5.3621 \mathrm{E}-03$ & $5.2021 \mathrm{E}-03$ & 5.4819E-03 & $5.9593 \mathrm{E}-03$ \\
\hline 4.00E-02 & $5.1278 \mathrm{E}-03$ & 4.7654E-03 & 4.6568E-03 & 4.9395E-03 & $5.4003 \mathrm{E}-03$ \\
\hline $3.00 \mathrm{E}-02$ & $2.0759 \mathrm{E}-03$ & $1.9324 \mathrm{E}-03$ & $1.8998 \mathrm{E}-03$ & $2.0264 \mathrm{E}-03$ & $2.2263 \mathrm{E}-03$ \\
\hline 2.53E-02 & $4.7235 \mathrm{E}-03$ & $4.4056 \mathrm{E}-03$ & 4.3795E-03 & 4.7204E-03 & 5.2342E-03 \\
\hline $1.00 \mathrm{E}-02$ & 4.0214E-04 & $3.7567 \mathrm{E}-04$ & $3.7910 \mathrm{E}-04$ & $4.1461 \mathrm{E}-04$ & $4.6586 \mathrm{E}-04$ \\
\hline $7.50 \mathrm{E}-03$ & $4.0006 \mathrm{E}-04$ & $3.7390 \mathrm{E}-04$ & $3.8150 \mathrm{E}-04$ & $4.2187 \mathrm{E}-04$ & $4.7886 \mathrm{E}-04$ \\
\hline $3.00 \mathrm{E}-03$ & 5.5627E-05 & 5.1989E-05 & 5.4307E-05 & $6.1555 \mathrm{E}-05$ & $7.1549 \mathrm{E}-05$ \\
\hline
\end{tabular}

Table 3.20: Change in flux as burnup increases. 


\begin{tabular}{|c|c|c|c|c|}
\hline Nuclide & $\begin{array}{c}\text { Reaction } \\
\text { Type }\end{array}$ & $\begin{array}{c}\text { Relative } \\
\text { Variance } \\
\text { for BOL Flux }\end{array}$ & $\begin{array}{c}\text { Relative } \\
\text { Variance } \\
\text { for Averaged } \\
\text { Flux }\end{array}$ & $\begin{array}{c}\text { Relative Variance } \\
\text { for BU Averaged } \\
\text { Flux }\end{array}$ \\
\hline 92235 & (Fission) & 2.479E-05 & 2.327E-05 & 2.341E-05 \\
\hline 92235 & $(n, \gamma)$ & $1.343 \mathrm{E}-03$ & $1.332 \mathrm{E}-03$ & $1.335 \mathrm{E}-03$ \\
\hline 92236 & (Fission) & 3.619E-04 & $3.413 \mathrm{E}-04$ & 3.373E-04 \\
\hline 92236 & $(n, \gamma)$ & $2.469 \mathrm{E}-03$ & $2.271 \mathrm{E}-03$ & $2.233 \mathrm{E}-03$ \\
\hline 92238 & $(n, 2 n)$ & 7.371E-03 & $8.136 \mathrm{E}-03$ & $8.366 \mathrm{E}-03$ \\
\hline 92238 & $(n, 3 n)$ & 1.442E-02 & 1.627E-02 & 1.684E-02 \\
\hline 92238 & (Fission) & $4.245 \mathrm{E}-04$ & $4.365 \mathrm{E}-04$ & $4.398 \mathrm{E}-04$ \\
\hline 92238 & $(n, y)$ & 9.982E-04 & 1.024E-03 & 1.033E-03 \\
\hline 93237 & (Fission) & $8.608 \mathrm{E}-03$ & $8.764 \mathrm{E}-03$ & 8.807E-03 \\
\hline 94238 & (Fission) & $8.105 E-03$ & $8.151 \mathrm{E}-03$ & $8.205 E-03$ \\
\hline 94238 & $(n, y)$ & $8.151 \mathrm{E}-04$ & 7.013E-04 & 7.182E-04 \\
\hline 94239 & (Fission) & 6.927E-05 & $4.575 \mathrm{E}-05$ & 4.275E-05 \\
\hline 94239 & $(n, y)$ & $6.489 \mathrm{E}-04$ & 3.999E-04 & 3.659E-04 \\
\hline 94240 & (Fission) & 2.087E-03 & $1.962 \mathrm{E}-03$ & $1.928 \mathrm{E}-03$ \\
\hline 94240 & $(n, \gamma)$ & $1.008 \mathrm{E}-03$ & 7.567E-04 & $6.873 \mathrm{E}-04$ \\
\hline 94241 & (Fission) & 6.947E-05 & 5.915E-05 & 5.859E-05 \\
\hline 94241 & $(n, y)$ & $1.668 \mathrm{E}-03$ & 1.233E-03 & $1.210 \mathrm{E}-03$ \\
\hline 94242 & $(n, 2 n)$ & 5.685E-02 & $6.301 \mathrm{E}-02$ & $6.487 \mathrm{E}-02$ \\
\hline 94242 & $(n, 3 n)$ & $2.474 \mathrm{E}-01$ & $2.791 \mathrm{E}-01$ & $2.889 \mathrm{E}-01$ \\
\hline 94242 & (Fission) & 1.529E-03 & 1.562E-03 & $1.571 \mathrm{E}-03$ \\
\hline 94242 & $(n, \gamma)$ & $5.200 \mathrm{E}-03$ & $5.168 \mathrm{E}-03$ & 5.147E-03 \\
\hline 95241 & $(n, 2 n)$ & $9.176 \mathrm{E}-01$ & $1.023 \mathrm{E}+00$ & $1.056 \mathrm{E}+00$ \\
\hline 95241 & $(n, 3 n)$ & 9.938E-01 & $1.121 \mathrm{E}+00$ & $1.160 \mathrm{E}+00$ \\
\hline 95241 & (Fission) & $4.635 \mathrm{E}-04$ & $4.583 \mathrm{E}-04$ & $4.573 \mathrm{E}-04$ \\
\hline 95241 & $(n, y)$ & 5.538E-05 & 4.694E-05 & $4.486 \mathrm{E}-05$ \\
\hline 95243 & (Fission) & $3.918 \mathrm{E}-03$ & 3.987E-03 & 4.007E-03 \\
\hline 95243 & $(n, \gamma)$ & 4.273E-01 & 3.207E-01 & 2.913E-01 \\
\hline
\end{tabular}

Table 3.21: Change in 1-group cross section uncertainty due to flux change. 


\begin{tabular}{|l|c|c|c|c|}
\hline & \multicolumn{4}{|c|}{ \% Standard Deviation } \\
\cline { 2 - 5 } Nuclide & ORIGEN & $\begin{array}{c}\text { ORIGEN } \\
\text { Averaged }\end{array}$ & $\begin{array}{c}\text { ORIGEN - BU } \\
\text { Averaged }\end{array}$ & TRITON \\
\hline pb210 & 1.195 & 1.115 & 1.106 & 0.152 \\
\hline ra226 & 1.355 & 1.262 & 1.252 & 0.185 \\
\hline ac227 & 0.480 & 0.453 & 0.454 & 0.231 \\
\hline th227 & 0.480 & 0.453 & 0.454 & 0.233 \\
\hline th230 & 1.491 & 1.394 & 1.384 & 0.185 \\
\hline pa231 & 0.518 & 0.490 & 0.494 & 0.253 \\
\hline u234 & 1.579 & 1.484 & 1.474 & 0.173 \\
\hline u235 & 1.312 & 1.316 & 1.339 & 0.583 \\
\hline u236 & 0.712 & 0.622 & 0.642 & 1.048 \\
\hline u237 & 2.521 & 2.154 & 2.248 & 2.053 \\
\hline u238 & 0.078 & 0.077 & 0.077 & 0.015 \\
\hline np237 & 0.605 & 0.566 & 0.544 & 1.602 \\
\hline np239 & 13.643 & 11.240 & 10.889 & 3.910 \\
\hline pu238 & 1.050 & 1.014 & 0.955 & 1.751 \\
\hline pu239 & 0.806 & 0.651 & 0.614 & 1.045 \\
\hline pu240 & 2.566 & 2.263 & 2.298 & 2.490 \\
\hline pu241 & 2.521 & 2.154 & 2.248 & 2.053 \\
\hline pu242 & 2.598 & 2.450 & 2.426 & 3.907 \\
\hline am241 & 2.502 & 2.137 & 2.233 & 2.053 \\
\hline am242m & 2.172 & 1.855 & 1.968 & 2.104 \\
\hline am243 & 13.643 & 11.240 & 10.889 & 3.908 \\
\hline cm242 & 2.172 & 1.854 & 1.968 & 2.107 \\
\hline cm244 & 11.340 & 9.238 & 9.015 & 4.345 \\
\hline cm245 & 10.251 & 8.297 & 8.138 & 4.680 \\
\hline c 14 & 0.452 & 0.446 & 0.411 & 0.379 \\
\hline se 79 & 0.366 & 0.355 & 0.344 & 0.088 \\
\hline sr 90 & 0.375 & 0.362 & 0.359 & 0.117 \\
\hline tc 99 & 1.970 & 1.903 & 1.847 & 0.075 \\
\hline i129 & 0.419 & 0.413 & 0.388 & 0.241 \\
\hline cs137 & 0.382 & 0.371 & 0.354 & 0.032 \\
\hline ba137m & 0.382 & 0.371 & 0.354 & 0.032 \\
\hline y 90 & 0.375 & 0.362 & 0.359 & 0.107 \\
\hline cs134 & 1.138 & 1.204 & 1.105 & 0.300 \\
\hline
\end{tabular}

Table 3.22: Comparing discharge isotopic uncertainties for various flux updates.

The primary cause is due to the intrinsic methodology within TRITON itself, making this cause less tangible than the previous data. When working with the simplified ORIGEN models, the cross section library used and perturbed was a working library that had already been updated by SAS2H. What occurs in updating is that the reference cross sections of the 
master library are subjected to resonance self-shielding analysis and updated based on those procedures. Consider Table 3.23 which shows the infinitely dilute, 44-group Am-243 capture cross section and the same 44-group cross section after resonance treatment is applied. Notice in the fast region, for example, some cross sections have changed by up to two orders of magnitude, while thermal energy groups show almost no change. The cross sections in the working library used by the lattice physics codes consisted of two parts: the reference component and the resonance self-shielded component in the resolved resonance energy range. When introducing perturbations into the cross sections in the master library, only the reference cross sections are perturbed. In the thermal energy range and unresolved resonances energy ranges, this perturbation is picked up because cross sections in these energy ranges were not considered in the resonance self-shielding analysis. However in the resonance regions, perturbations in the reference cross sections are easily overwhelmed by the magnitude of the resonance updates. This accounts for the smaller values of uncertainty seen in the TRITON models. This in turn forces the assumption that the resonances are not perturbed at all which means they are assumed to be perfectly known. This assumption can lead to under-estimated uncertainties, e.g. plutonium is highly affected by low-lying resonances in the U-238 absorption cross section. Further study into this matter was beyond the scope of this work. 


\begin{tabular}{|c|c|c|}
\hline Energy & Master Cross Section & Treated Cross Section \\
\hline $2.00 \mathrm{E}+07$ & 1.10580996E-02 & $2.51107000 \mathrm{E}+00$ \\
\hline $8.19 E+06$ & 1.15339998E-02 & $2.44640000 \mathrm{E}+00$ \\
\hline $6.43 E+06$ & 1.23779997E-02 & $1.80170000 \mathrm{E}+00$ \\
\hline $4.80 E+06$ & $1.90602001 \mathrm{E}-02$ & $1.58490000 \mathrm{E}+00$ \\
\hline $3.00 E+06$ & 3.09389997E-02 & $1.57700000 \mathrm{E}+00$ \\
\hline $2.48 E+06$ & $3.72469984 \mathrm{E}-02$ & $1.57860000 \mathrm{E}+00$ \\
\hline $2.35 \mathrm{E}+06$ & $4.79950011 \mathrm{E}-02$ & $1.66690000 \mathrm{E}+00$ \\
\hline $1.85 \mathrm{E}+06$ & $7.02812970 \mathrm{E}-02$ & $1.63750000 \mathrm{E}+00$ \\
\hline $1.40 \mathrm{E}+06$ & $1.07110001 \mathrm{E}-01$ & $1.45130000 \mathrm{E}+00$ \\
\hline $9.00 \mathrm{E}+05$ & $2.26100996 \mathrm{E}-01$ & 4.88705000E-01 \\
\hline 4.00E+05 & $5.77825010 \mathrm{E}-01$ & $5.96831000 \mathrm{E}-01$ \\
\hline $1.00 \mathrm{E}+05$ & $1.56948996 \mathrm{E}+00$ & $1.58408000 \mathrm{E}+00$ \\
\hline $2.50 E+04$ & $2.11269999 \mathrm{E}+00$ & $2.12890000 \mathrm{E}+00$ \\
\hline $1.70 \mathrm{E}+04$ & $3.18284011 \mathrm{E}+00$ & $3.19814000 \mathrm{E}+00$ \\
\hline $3.00 E+03$ & $8.80949974 \mathrm{E}+00$ & $8.81098000 \mathrm{E}+00$ \\
\hline $5.50 E+02$ & $2.44122009 \mathrm{E}+01$ & $2.44834000 \mathrm{E}+01$ \\
\hline $1.00 \mathrm{E}+02$ & $4.30122986 \mathrm{E}+01$ & $4.36353000 \mathrm{E}+01$ \\
\hline $3.00 \mathrm{E}+01$ & 1.03167999E+02 & $1.02405000 \mathrm{E}+02$ \\
\hline $1.00 \mathrm{E}+01$ & $3.96268997 \mathrm{E}+01$ & $4.01052000 \mathrm{E}+01$ \\
\hline $8.10 E+00$ & $2.73347992 \mathrm{E}+02$ & $2.98206000 \mathrm{E}+02$ \\
\hline $6.00 \mathrm{E}+00$ & $1.06544998 \mathrm{E}+02$ & $1.05959000 \mathrm{E}+02$ \\
\hline $4.75 E+00$ & 1.19114998E+02 & $1.19325000 \mathrm{E}+02$ \\
\hline $3.00 E+00$ & $9.09586029 \mathrm{E}+01$ & $1.03057000 \mathrm{E}+02$ \\
\hline 1.77E+00 & $2.30728003 \mathrm{E}+03$ & $2.27778000 \mathrm{E}+03$ \\
\hline $1.00 \mathrm{E}+00$ & 1.05037003E+02 & $1.04765000 \mathrm{E}+02$ \\
\hline $6.25 \mathrm{E}-01$ & $4.81402016 \mathrm{E}+01$ & $4.75895000 \mathrm{E}+01$ \\
\hline 4.00E-01 & $5.20870018 \mathrm{E}+01$ & $5.45036000 \mathrm{E}+01$ \\
\hline 3.75E-01 & $3.81459999 \mathrm{E}+01$ & $3.93748000 \mathrm{E}+01$ \\
\hline $3.50 \mathrm{E}-01$ & $3.39099998 \mathrm{E}+01$ & $3.42099000 \mathrm{E}+01$ \\
\hline 3.25E-01 & $3.19234009 \mathrm{E}+01$ & $3.19722000 E+01$ \\
\hline 2.75E-01 & $3.14440002 \mathrm{E}+01$ & $3.14953000 \mathrm{E}+01$ \\
\hline $2.50 \mathrm{E}-01$ & $3.16469994 \mathrm{E}+01$ & $3.16905000 \mathrm{E}+01$ \\
\hline $2.25 \mathrm{E}-01$ & $3.21749992 \mathrm{E}+01$ & $3.21896000 \mathrm{E}+01$ \\
\hline 2.00E-01 & $3.37787018 \mathrm{E}+01$ & $3.34703000 \mathrm{E}+01$ \\
\hline $1.50 \mathrm{E}-01$ & $3.78905983 \mathrm{E}+01$ & $3.79820000 \mathrm{E}+01$ \\
\hline $1.00 \mathrm{E}-01$ & $4.37220001 \mathrm{E}+01$ & $4.38877000 \mathrm{E}+01$ \\
\hline 7.00E-02 & $5.05588989 \mathrm{E}+01$ & $5.06487000 \mathrm{E}+01$ \\
\hline $5.00 \mathrm{E}-02$ & $5.72869987 \mathrm{E}+01$ & $5.72870000 E+01$ \\
\hline 4.00E-02 & $6.43789978 \mathrm{E}+01$ & $6.43790000 \mathrm{E}+01$ \\
\hline $3.00 \mathrm{E}-02$ & $7.17419968 \mathrm{E}+01$ & $7.17420000 E+01$ \\
\hline 2.53E-02 & $9.00500031 \mathrm{E}+01$ & $9.00490000 \mathrm{E}+01$ \\
\hline $1.00 \mathrm{E}-02$ & $1.25260002 \mathrm{E}+02$ & $1.25260000 \mathrm{E}+02$ \\
\hline $7.50 \mathrm{E}-03$ & $1.60410004 \mathrm{E}+02$ & $1.60868000 \mathrm{E}+02$ \\
\hline $3.00 \mathrm{E}-03$ & $2.79713013 \mathrm{E}+02$ & $2.85547000 \mathrm{E}+02$ \\
\hline
\end{tabular}

Table 3.23: Change in Am-243 capture cross section due to resonance treatment. 


\subsubsection{Fast Reactor Models with Transuranic Fuels}

Before discussing the fast reactor models, it is important to take an objective look at the capabilities of TRITON, and to recognize how these may limit the results. TRITON is part of SCALE, which, for the most part was designed with current LWR's in mind. The most restrictive issue is with TRITON's cell domain restrictions. While any polygon can be modeled inside the cell, the cell itself is required to be rectangular, and the remaining space must be filled with moderator. For BWR and PWR square assemblies this is fine - one can model the exact dimensions of either the Wigner cell or the entire assembly. However if one tries to input a hexagonal cell, like the ones in the following models, the exact dimensions cannot be modeled. Essentially one ends up with a hexagonal peg in a square hole, which is filled with additional coolant, which yields an over moderated cell, which in turn affects flux which, in turn affects isotopic depletion. Another restriction is that we are using the 44group cross-section library which has a corresponding 44-group covariance library. These cross-sections were generated for a thermal reactor, i.e. about $50 \%$ of the data is in the thermal groups. There are only a few, broad fast energy groups, whose cross-sections are the reactions which drive the fast reactor. So, this over moderated cell and lack of fine data in the region where most reactions occur forces one to question the results obtained using this method. Further, it was early noted that the uncertainty of the resonances could not be treated. While the results do clearly demonstrate the methodology developed in this work, the actual numerical values can only be taken as plausible, rather than absolutely accurate. To provide comparison, Argonne's REBUS fast reactor code was used to further examine one of the fast reactor models. The fuel design corresponding to a conversion ratio of 0.70 is 
modeled in REBUS and those results are presented in a subsequent section following the TRITON models.

Three distinctly different fast reactor models were examined in TRITON. Physically, they differ in terms of composition and operating conditions, but those parameters cause a difference in another key property of the fuel - conversion ratio or CR. For this study, the fuels examined have CRs equal to $0.25,0.70$ and 1.05 , the latter being a so called breeder reactor and the two former being burner reactors. A description of basic composition (Table 3.24) and geometry and operating conditions (Table 3.25) of each is presented below, but the reader is referred to Appendix A where more detailed model data are provided. Table 3.26 through Table 3.28 show the discharge isotopics and Table 3.29 presents the isotopic relative standard deviations for each fuel type, in order of ascending conversion ratio. The reader will observe that U-235 content does not monotonically change between conversion ratios as do other isotopics. This anomalous behavior is noted but the source could not be identified within this work. The reader will also observe that uncertainty increases with conversion ratio. Increasing conversion ratio requires increasing uranium content and the relative fissile fraction of TRU. In observing the values in the SCALE covariance library, the largest sources of uncertainty are the fission and absorption reactions of the fissile minor actinides, which are often correlated to U-235. It follows that the increase in uranium and fissile TRU faction serve to magnify these uncertainties, thus uncertainty should increase as conversion ratio increases, which is the observed behavior. 


\begin{tabular}{|c|c|c|c|}
\hline \multirow[b]{2}{*}{ Nuclide / Conversion Ratio: } & \multicolumn{3}{|c|}{ Weight Percent in TRU } \\
\hline & 0.25 & 0.7 & 1.05 \\
\hline $\mathrm{Np}-237$ & 18.635 & 7.334 & 9.907 \\
\hline $\mathrm{Pu}-238$ & 0.855 & 1.253 & 0.000 \\
\hline Pu-239 & 32.764 & 48.058 & 72.150 \\
\hline Pu-240 & 14.983 & 21.973 & 4.469 \\
\hline Pu-241 & 4.936 & 7.241 & 0.250 \\
\hline $\mathrm{Pu}-242$ & 2.956 & 4.335 & 0.000 \\
\hline Am-241 & 20.579 & 8.100 & 10.941 \\
\hline$A m-242 m$ & 0.041 & 0.016 & 0.022 \\
\hline Am-243 & 3.565 & 1.403 & 1.895 \\
\hline Cm-244 & 0.689 & 0.271 & 0.366 \\
\hline Cm-245 & 0.041 & 0.016 & 0.022 \\
\hline Fissile Fraction, $\%$ & 37.7 & 55.30 & 72.40 \\
\hline TRU Enrichment, \% & 59.2 & 20.6 & 16.2 \\
\hline Zr w/o & 20 & 10 & 10 \\
\hline Depleted U, w/o & 20.8 & 69.4 & 73.8 \\
\hline
\end{tabular}

Table 3.24: Fuel composition data for fast reactor models.

\begin{tabular}{|l|r|r|r|}
\hline Conversion Ration & $\mathbf{0 . 2 5}$ & $\mathbf{0 . 7 0}$ & $\mathbf{1 . 0 5}$ \\
\hline $\begin{array}{l}\text { Specific Power of active core, } \\
\text { MW/MT }\end{array}$ & 114.8 & 47.7 & 41.2 \\
\hline Discharge Burnup, GWD.MT & 94.3 & 78.4 & 67.7 \\
\hline Height, cm & 80 & 80 & 80 \\
\hline Number of pins per assembly & 217 & 169 & 127 \\
\hline Assembly lattice pitch, cm & 14.834 & 14.834 & 14.834 \\
\hline Inter-assembly gap, mm & 4.45 & 4.0 & 4.0 \\
\hline Duct thickness, mm & 4.45 & 3.0 & 3.0 \\
\hline Pin pitch-to-diameter ratio & 1.29 & 1.11 & 1.10 \\
\hline Cladding thickness, mm & 0.75 & 0.41 & 0.41 \\
\hline
\end{tabular}

Table 3.25: Fuel geometry and power data for fast reactor models. 


\begin{tabular}{|c|c|c|c|c|c|}
\hline \multicolumn{6}{|c|}{ Discharge Isotopics, grams / MTHM } \\
\hline pb210 & $4.950 \mathrm{E}-10$ & np237 & $9.563 \mathrm{E}+03$ & cm244 & $1.268 \mathrm{E}+04$ \\
\hline ra226 & 4.002E-09 & np239 & $1.147 \mathrm{E}+02$ & cm245 & $3.075 \mathrm{E}+03$ \\
\hline ac227 & 1.967E-09 & pu238 & $2.691 \mathrm{E}+04$ & c 14 & $9.454 \mathrm{E}-03$ \\
\hline th227 & $2.759 \mathrm{E}-11$ & pu239 & $1.367 \mathrm{E}+05$ & se 79 & $1.023 \mathrm{E}+01$ \\
\hline th230 & $4.585 \mathrm{E}-04$ & pu240 & $1.934 \mathrm{E}+05$ & sr 90 & $7.015 \mathrm{E}+02$ \\
\hline pa231 & $4.248 \mathrm{E}-04$ & pu241 & $3.548 \mathrm{E}+04$ & tc 99 & $2.454 \mathrm{E}+03$ \\
\hline u234 & $4.204 \mathrm{E}+02$ & pu242 & $5.692 \mathrm{E}+04$ & i129 & $5.747 \mathrm{E}+02$ \\
\hline u235 & $5.135 \mathrm{E}+02$ & am241 & $2.383 E+04$ & cs137 & $3.582 \mathrm{E}+03$ \\
\hline u236 & $1.228 \mathrm{E}+02$ & am242m & $1.908 \mathrm{E}+03$ & ba137m & $5.560 \mathrm{E}-04$ \\
\hline u237 & $9.338 \mathrm{E}-01$ & am243 & $1.807 \mathrm{E}+04$ & y90 & $2.008 \mathrm{E}-01$ \\
\hline u238 & $3.789 \mathrm{E}+05$ & cm242 & $2.045 \mathrm{E}+03$ & cs134 & $1.694 \mathrm{E}+02$ \\
\hline
\end{tabular}

Table 3.26: Discharge isotopics for fast reactor fuel of $\mathrm{CR}=0.25$.

\begin{tabular}{|l|l|l|l|l|r|}
\hline \multicolumn{7}{|c|}{ Discharge Isotopics, grams / MTHM } \\
\hline pb210 & $3.200 \mathrm{E}-10$ & np237 & $1.895 \mathrm{E}+03$ & cm244 & $2.249 \mathrm{E}+03$ \\
\hline ra226 & $3.978 \mathrm{E}-09$ & np239 & $1.288 \mathrm{E}+02$ & cm245 & $5.264 \mathrm{E}+02$ \\
\hline ac227 & $1.573 \mathrm{E}-09$ & pu238 & $5.555 \mathrm{E}+03$ & c 14 & $9.724 \mathrm{E}-03$ \\
\hline th227 & $1.118 \mathrm{E}-11$ & pu239 & $9.083 \mathrm{E}+04$ & se 79 & $8.726 \mathrm{E}+00$ \\
\hline th230 & $2.281 \mathrm{E}-04$ & pu240 & $6.292 \mathrm{E}+04$ & sr 90 & $6.166 \mathrm{E}+02$ \\
\hline pa231 & $2.595 \mathrm{E}-04$ & pu241 & $9.851 \mathrm{E}+03$ & tc 99 & $2.040 \mathrm{E}+03$ \\
\hline u234 & $1.632 \mathrm{E}+02$ & pu242 & $1.066 \mathrm{E}+04$ & i129 & $4.561 \mathrm{E}+02$ \\
\hline u235 & $6.758 \mathrm{E}+02$ & am241 & $4.796 \mathrm{E}+03$ & cs137 & $2.878 \mathrm{E}+03$ \\
\hline $\mathbf{u 2 3 6}$ & $2.213 \mathrm{E}+02$ & am242m & $3.738 \mathrm{E}+02$ & ba137m & $4.465 \mathrm{E}-04$ \\
\hline $\mathbf{u 2 3 7}$ & $1.576 \mathrm{E}+00$ & am243 & $3.234 \mathrm{E}+03$ & y90 & $1.751 \mathrm{E}-01$ \\
\hline $\mathbf{u 2 3 8}$ & $7.246 \mathrm{E}+05$ & $\mathbf{c m 2 4 2}$ & $3.251 \mathrm{E}+02$ & cs134 & $1.704 \mathrm{E}+02$ \\
\hline
\end{tabular}

Table 3.27: Discharge isotopics for fast reactor fuel of $\mathrm{CR}=\mathbf{0 . 7 0}$

\begin{tabular}{|l|l|l|l|l|r|}
\hline \multicolumn{7}{|c|}{ Discharge Isotopics, grams / MTHM } \\
\hline pb210 & $8.775 \mathrm{E}-11$ & np237 & $5.698 \mathrm{E}+02$ & cm244 & $2.776 \mathrm{E}+02$ \\
\hline ra226 & $1.135 \mathrm{E}-09$ & np239 & $1.438 \mathrm{E}+02$ & cm245 & $6.964 \mathrm{E}+01$ \\
\hline ac227 & $4.121 \mathrm{E}-10$ & pu238 & $1.370 \mathrm{E}+03$ & c 14 & $1.071 \mathrm{E}-02$ \\
\hline th227 & $2.723 \mathrm{E}-12$ & pu239 & $8.565 \mathrm{E}+04$ & se 79 & $8.740 \mathrm{E}+00$ \\
\hline th230 & $5.873 \mathrm{E}-05$ & pu240 & $3.916 \mathrm{E}+04$ & sr 90 & $6.242 \mathrm{E}+02$ \\
\hline pa231 & $6.911 \mathrm{E}-05$ & pu241 & $5.607 \mathrm{E}+03$ & tc 99 & $2.013 \mathrm{E}+03$ \\
\hline u234 & $4.292 \mathrm{E}+01$ & pu242 & $2.129 \mathrm{E}+03$ & i129 & $4.408 \mathrm{E}+02$ \\
\hline $\mathbf{u 2 3 5}$ & $5.982 \mathrm{E}+02$ & am241 & $1.414 \mathrm{E}+03$ & cs137 & $2.814 \mathrm{E}+03$ \\
\hline u236 & $2.533 \mathrm{E}+02$ & am242m & $9.679 \mathrm{E}+01$ & ba137m & $4.372 \mathrm{E}-04$ \\
\hline u237 & $1.674 \mathrm{E}+00$ & am243 & $4.754 \mathrm{E}+02$ & y90 & $1.776 \mathrm{E}-01$ \\
\hline $\mathbf{u 2 3 8}$ & $7.826 \mathrm{E}+05$ & $\mathbf{c m 2 4 2}$ & $9.319 \mathrm{E}+01$ & cs134 & $1.890 \mathrm{E}+02$ \\
\hline
\end{tabular}

Table 3.28: Discharge isotopics for fast reactor fuel of $C R=1.05$. 


\begin{tabular}{|l|c|r|r|}
\hline \multirow{2}{*}{ Nuclide } & \multicolumn{3}{|c|}{ Conversion Ratio } \\
\cline { 2 - 4 } pb210 & $\mathbf{0 . 2 5}$ & $\mathbf{0 . 7}$ & \multicolumn{1}{|c|}{$\mathbf{1 . 0 5}$} \\
\hline ra225 & 2.770 & 2.668 & 3.029 \\
ac227 & 1.229 & 3.760 & 4.214 \\
th227 & 1.227 & 2.415 & 2.992 \\
th230 & 2.935 & 3.999 & 2.990 \\
pa231 & 1.222 & 2.384 & 2.431 \\
u234 & 3.129 & 4.317 & 4.723 \\
u235 & 1.490 & 2.587 & 3.267 \\
u236 & 1.795 & 4.793 & 5.535 \\
u237 & 2.764 & 3.832 & 4.247 \\
u238 & 0.205 & 0.230 & 0.255 \\
np237 & 1.114 & 1.680 & 2.828 \\
np239 & 10.752 & 15.475 & 15.989 \\
pu238 & 3.371 & 4.824 & 5.204 \\
pu239 & 1.468 & 2.406 & 2.800 \\
pu240 & 0.906 & 1.992 & 3.066 \\
pu241 & 2.764 & 3.833 & 4.246 \\
pu242 & 0.548 & 1.150 & 3.141 \\
am241 & 1.341 & 2.369 & 3.271 \\
am242m & 1.434 & 1.836 & 1.972 \\
am243 & 10.752 & 15.475 & 15.989 \\
cm242 & 1.434 & 1.837 & 1.972 \\
cm244 & 13.025 & 16.925 & 19.875 \\
cm245 & 4.344 & 9.295 & 11.948 \\
c 14 & 1.655 & 0.962 & 0.708 \\
se 79 & 1.137 & 0.627 & 0.544 \\
sr 90 & 0.348 & 0.180 & 0.159 \\
tc 99 & 0.319 & 0.472 & 0.554 \\
i129 & 0.412 & 0.460 & 0.494 \\
cs137 & 0.094 & 0.098 & 0.099 \\
ba137m & 0.094 & 0.092 & 0.099 \\
y 90 & 0.348 & 0.181 & 0.158 \\
cs134 & 2.553 & 2.250 & 2.214 \\
\hline
\end{tabular}

Table 3.29: Relative isotopic uncertainties for fast reactor fuels.

\subsubsection{Fast Reactor Model with Transuranic Fuel and Recycling}

The principles of the recycling methodology have already been discussed, and it suffices to say that the model used in this experiment is the same as the $\mathrm{CR}=0.70$ model discussed in the previous section, save for the fact that the model burnup will be adjusted to give the end of cycle burnup of $41.4 \mathrm{GWD} / \mathrm{MTHM}$ rather then the end of life burnup 
modeled in the previous section. This is done so that the target k-effective will the one for end of cycle, which is what would be real world objective of the reactor operator and fuel designer. Also, as indicated previously, the method of choice is the UREX process in which transuranics are separated as a stream and combined with depleted uranium to make up the new recycled fuel; however one could use any separation scheme with the methodology described. The model was run to an equilibrium state at 6 recycles and, in addition to typical uncertainty data, the beginning of cycle and end of cycle k-effective values, and their uncertainties, were also collected in output data. The values for end of cycle k-effective were also used to adjust transuranics loading with each recycle to maintain cycle energy production. Since the composition is defined by elemental weight percents and volume fractions, those are compared in Table 3.30. Table 3.31 gives the discharge isotopics of the equilibrium model and Table 3.32 shows discharge isotopics uncertainties originating due to cross sections, recycled isotopics, and total combined uncertainties. As can easily be seen from just the isotopics uncertainties, recycled isotopics originated uncertainties add to the cross-sections originated uncertainties to give almost a two-fold increase in total discharge isotopics uncertainties. A simple study (not shown) was conducted where it is assumed that the recycled fast reactor composition is known. When only the uncertainties of the LWR fuel were applied, this being a small fraction of the fuel in this particular TRITON model and possessing small uncertainties, the uncertainties originating from recycled isotopics in this case were negligible, (which is why they were not presented). In reality the reprocessing engineer knows fairly accurately the composition of the spent fast reactor and LWR fuels via performing mass spectroscopy. Knowing these compositions, the mass fractions of recycled fast reactor fuel, thermal reactor fuel, and depleted uranium would be altered to assure the 
target EOC k-effective value is predicted to be achieved based upon cross section values which have inaccuracies.

\begin{tabular}{|c|c|c|c|}
\hline \multicolumn{4}{|c|}{ Fuel Composition Properties } \\
\hline & & No Recycle & Equilibirum \\
\hline \multicolumn{2}{|c|}{ Uranium Volume Fraction: } & 0.5682 & 0.5594 \\
\hline $\begin{array}{l}\text { U-235 } \\
\text { U-238 }\end{array}$ & $\begin{array}{l}(w / o) \\
(w / o)\end{array}$ & $\begin{array}{r}0.200 \\
99.800\end{array}$ & $\begin{array}{r}0.200 \\
99.800\end{array}$ \\
\hline \multicolumn{2}{|c|}{ Neptunium Volume Fraction: } & 0.0023 & 0.001 \\
\hline $\mathrm{Np}-237$ & $(w / 0)$ & 100.000 & 100.000 \\
\hline \multicolumn{2}{|c|}{ Plutonium Volume Fraction: } & 0.1283 & 0.1380 \\
\hline $\mathrm{Pu}-238$ & $(w / 0)$ & 1.512 & 2.972 \\
\hline Pu-239 & $(w / 0)$ & 57.999 & 47.048 \\
\hline Pu-240 & $(w / 0)$ & 26.518 & 37.937 \\
\hline Pu-241 & $(w / 0)$ & 8.739 & 5.715 \\
\hline Pu-242 & (w/o) & 5.232 & 6.929 \\
\hline \multicolumn{2}{|c|}{ Americium Volume Fraction: } & 0.0109 & 0.0108 \\
\hline Am-241 & $(w / 0)$ & 85.092 & 63.995 \\
\hline$A m-242 m$ & $(w / 0)$ & 0.168 & 3.574 \\
\hline Am-243 & (w/o) & 14.740 & 32.431 \\
\hline \multicolumn{2}{|c|}{ Curium Volume Fraction: } & 0.0022 & 0.0031 \\
\hline $\mathrm{Cm}-242$ & (w/o) & 0.000 & 1.170 \\
\hline $\mathrm{Cm}-244$ & $(w / 0)$ & 94.444 & 76.878 \\
\hline $\mathrm{Cm}-245$ & $(w / 0)$ & 5.556 & 21.952 \\
\hline $\mathrm{Cm}-246$ & $(w / 0)$ & 0.000 & 1.170 \\
\hline
\end{tabular}

Table 3.30: Comparison of fuel composition properties for once through and recycled fuel.

\begin{tabular}{|l|l|l|l|l|c|}
\hline \multicolumn{7}{|c|}{ Discharge Isotopics, grams / MTHM } \\
\hline pb210 & $7.407 \mathrm{E}-11$ & np237 & $1.172 \mathrm{E}+03$ & cm244 & $2.593 \mathrm{E}+03$ \\
\hline ra226 & $1.074 \mathrm{E}-09$ & np239 & $1.264 \mathrm{E}+02$ & cm245 & $7.121 \mathrm{E}+02$ \\
\hline ac227 & $5.675 \mathrm{E}-10$ & pu238 & $5.574 \mathrm{E}+03$ & c 14 & $4.817 \mathrm{E}-03$ \\
\hline th227 & $5.309 \mathrm{E}-12$ & pu239 & $8.999 \mathrm{E}+04$ & se 79 & $4.781 \mathrm{E}+00$ \\
\hline th230 & $1.214 \mathrm{E}-04$ & pu240 & $7.586 \mathrm{E}+04$ & sr 90 & $3.318 \mathrm{E}+02$ \\
\hline pa231 & $1.061 \mathrm{E}-04$ & pu241 & $1.201 \mathrm{E}+04$ & tc 99 & $1.093 \mathrm{E}+03$ \\
\hline $\mathbf{u 2 3 4}$ & $9.651 \mathrm{E}+01$ & pu242 & $1.257 \mathrm{E}+04$ & $\mathbf{i 1 2 9}$ & $2.591 \mathrm{E}+02$ \\
\hline u235 & $9.771 \mathrm{E}+02$ & am241 & $5.878 \mathrm{E}+03$ & cs137 & $1.560 \mathrm{E}+03$ \\
\hline u236 & $1.451 \mathrm{E}+02$ & am242m & $3.963 \mathrm{E}+02$ & ba137m & $2.418 \mathrm{E}-04$ \\
\hline u237 & $1.475 \mathrm{E}+00$ & am243 & $3.506 \mathrm{E}+03$ & y90 & $9.372 \mathrm{E}-02$ \\
\hline $\mathbf{u 2 3 8}$ & $7.452 \mathrm{E}+05$ & $\mathbf{c m 2 4 2}$ & $3.720 \mathrm{E}+02$ & cs134 & $5.866 \mathrm{E}+01$ \\
\hline
\end{tabular}

Table 3.31: Discharge isotopics for equilibrium recycled fuel. 


\begin{tabular}{|l|c|r|r|}
\hline Nuclide & $\begin{array}{c}\text { Recycled } \\
\text { Isotopics } \\
\text { Orignated } \\
\text { (At Equilibrium) }\end{array}$ & $\begin{array}{r}\text { Cross } \\
\text { Sections } \\
\text { Orignated }\end{array}$ & \multicolumn{1}{|c|}{ Total } \\
\hline pb210 & 12.450 & 2.668 & 12.732 \\
ra225 & 12.931 & 3.760 & 13.466 \\
ac227 & 14.025 & 2.415 & 14.231 \\
th227 & 14.026 & 2.415 & 14.232 \\
th230 & 12.681 & 3.999 & 13.296 \\
pa231 & 13.465 & 2.384 & 13.674 \\
u234 & 12.364 & 4.317 & 13.096 \\
u235 & 1.121 & 2.587 & 2.819 \\
u236 & 3.189 & 4.793 & 5.757 \\
u237 & 5.460 & 3.832 & 6.671 \\
u238 & 0.120 & 0.230 & 0.260 \\
np237 & 11.311 & 1.680 & 11.435 \\
np239 & 44.021 & 15.475 & 46.662 \\
pu238 & 11.931 & 4.824 & 12.869 \\
pu239 & 4.296 & 2.406 & 4.924 \\
pu240 & 5.107 & 1.992 & 5.482 \\
pu241 & 5.460 & 3.833 & 6.671 \\
pu242 & 6.325 & 1.150 & 6.429 \\
am241 & 6.846 & 2.369 & 7.244 \\
am242m & 9.100 & 1.836 & 9.284 \\
am243 & 44.021 & 15.475 & 46.662 \\
cm242 & 9.100 & 1.837 & 9.284 \\
cm244 & 9.202 & 16.925 & 19.265 \\
cm245 & 12.163 & 9.295 & 15.308 \\
c 14 & 2.886 & 0.962 & 3.042 \\
se 79 & 1.415 & 0.627 & 1.548 \\
sr90 & 0.369 & 0.180 & 0.410 \\
tc 99 & 0.204 & 0.472 & 0.514 \\
i129 & 0.719 & 0.460 & 0.854 \\
cs137 & 0.106 & 0.098 & 0.144 \\
ba137m & 0.106 & 0.092 & 0.140 \\
y 90 & 0.369 & 0.181 & 0.410 \\
cs134 & 1.932 & 2.250 & 2.966 \\
\hline
\end{tabular}

Table 3.32: Discharged isotopics uncertainties originating from recycled isotopics and cross sections sources of uncertainty. 


\subsubsection{Comparison of Results, TRITON Models}

As with the simplified models, the two most commonly examined metrics for repository performance or reprocessing - decay heat and radioactivity spanning 10 to 10,000 years of decay time, are graphically presented. These data are plots of the numerical values of the metrics, propagated from number density uncertainties presented in the previous sections for the TRITON models, including once through and the recycling method.

The first plots compare the uncertainty between the PWR models for the simplified ORIGEN method and the TRITON method (decay heat in Figure 3.11 and radioactivity in Figure 3.12). While, in terms of isotopics, the two methods deliver different uncertainties for reasons already discussed, as can clearly be seen, the long term affect on the metrics of interest is generally the same. This occurs mainly because the uncertainty on the long term heat contributors, e.g. plutonium, is on the same order of magnitude between the two models.

Next, the fast reactor models, three different compositions and conversion ratios, are compared with each other for the no recycle case. Since these are three different fuel types with different operating and composition parameters, comparison just provides a look at the three possibilities. While decay heat (Figure 3.13) and radioactivity (Figure 3.14) were higher for the low conversion ratio fuel, its long term uncertainty was the lowest. This is an interesting consideration for planning a fuel scenario regarding what one wants to dispose of and what one wants to recycle.

Finally, comparison is drawn between once-through faster reactor fuel and recycled fast reactor fuel, adding to it a stream of spent light water reactor fuel. As one would expect, uncertainty in both decay heat (Figure 3.15) and radioactivity (Figure 3.16) is higher in the 
recycled fuel than in the once-through, and these figures give a visual comparison of that difference. In general, recycling nearly doubled the amount of uncertainty on the metrics examined, though the nominal long term performance is nearly identical for the two cases. Also, the k-effective study shows that the uncertainties originating from cross sections are the greatest contributor to k-effective uncertainty in that method (Figure 3.18), but uncertainties originating from recycled isotopic uncertainties scheme (recycling uncertainties alone in Figure 3.17) add a noticeable increase to that uncertainty (Figure 3.18). As can be seen from these figures, uncertainty from recycling seems to increase to some saturation as equilibrium is reached. 


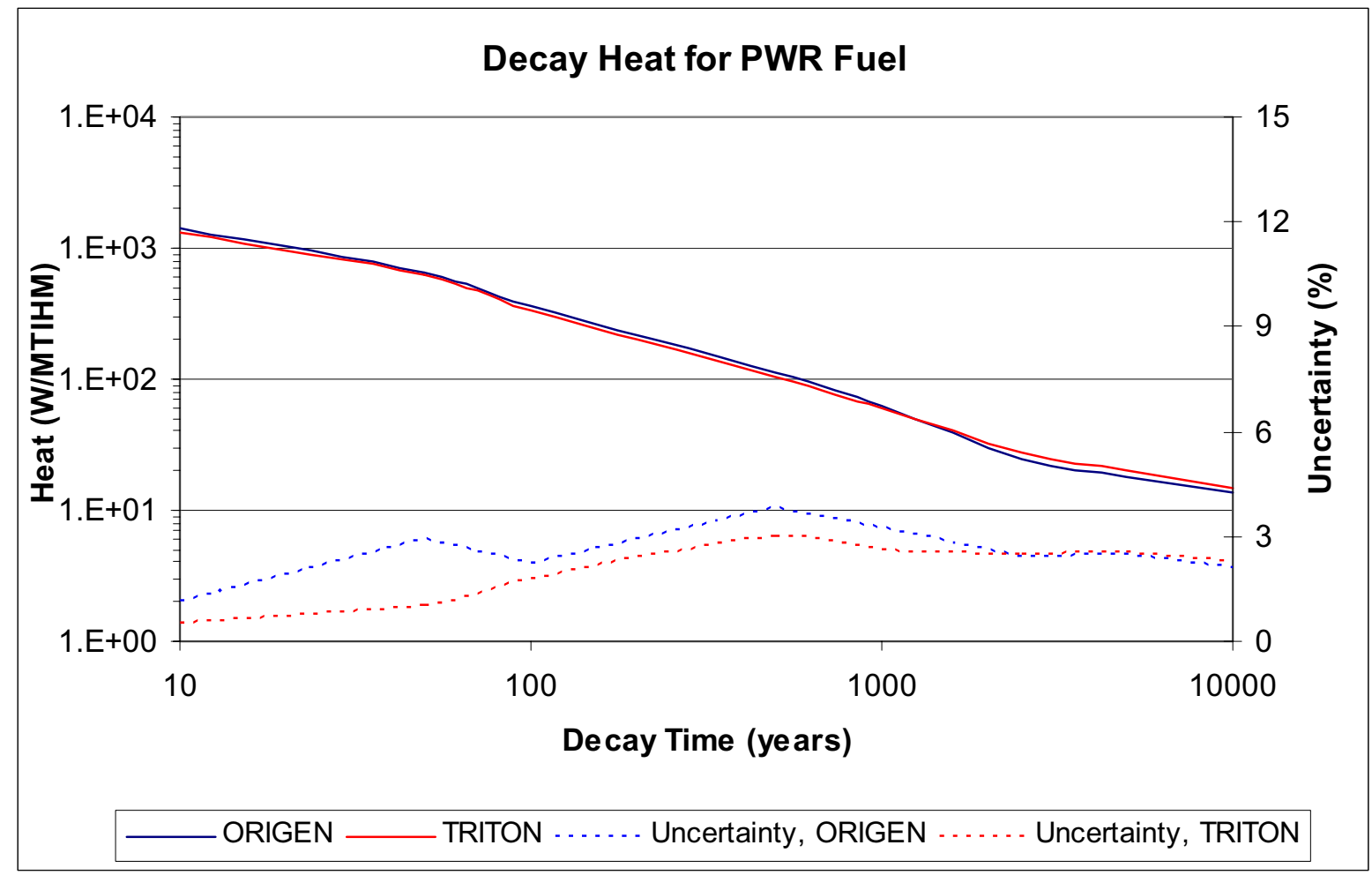

Figure 3.11: Decay heat comparison of simple ORIGEN and TRITON models.

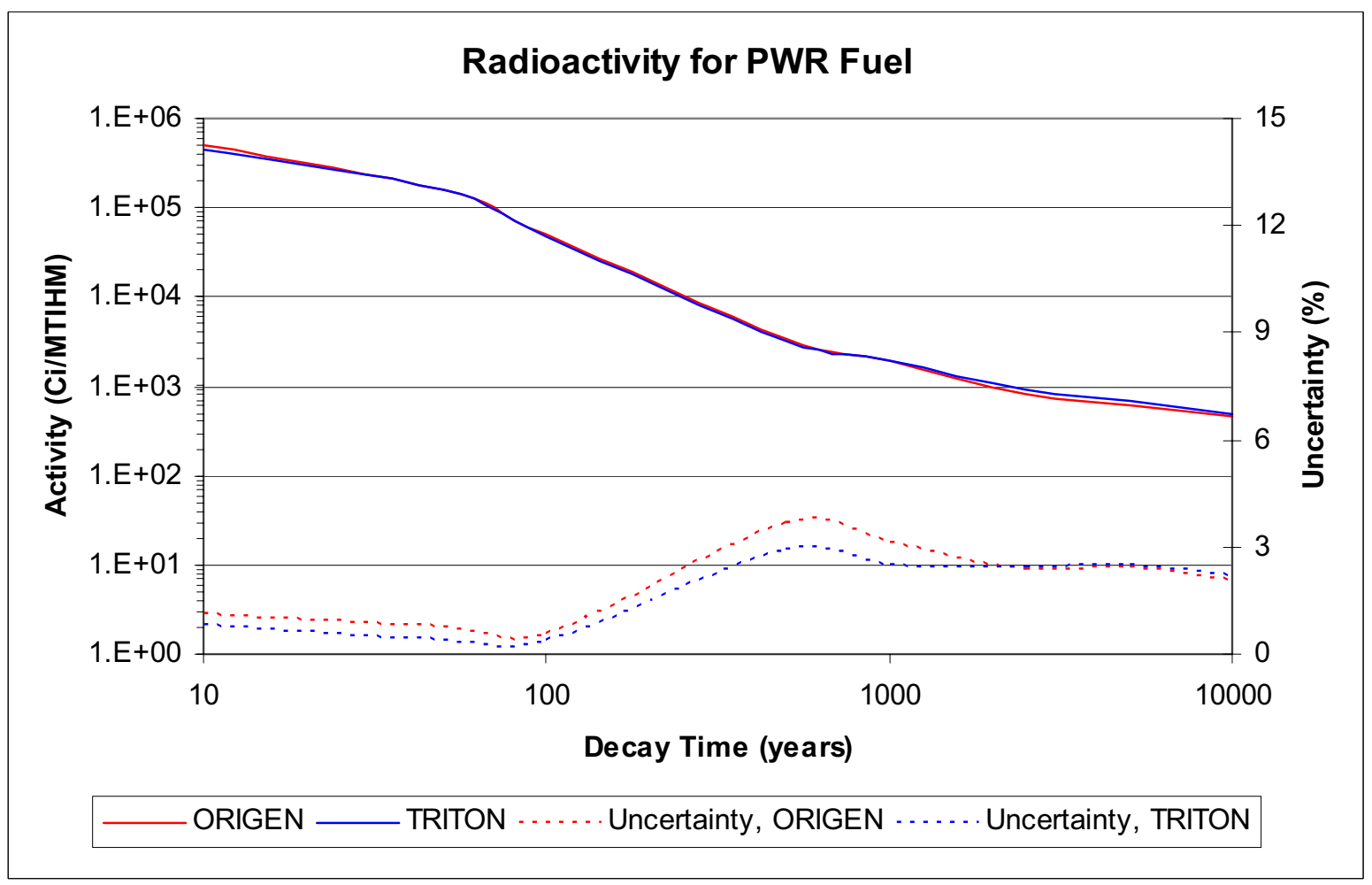

Figure 3.12: Radioactivity comparison of simple ORIGEN and TRITON models. 


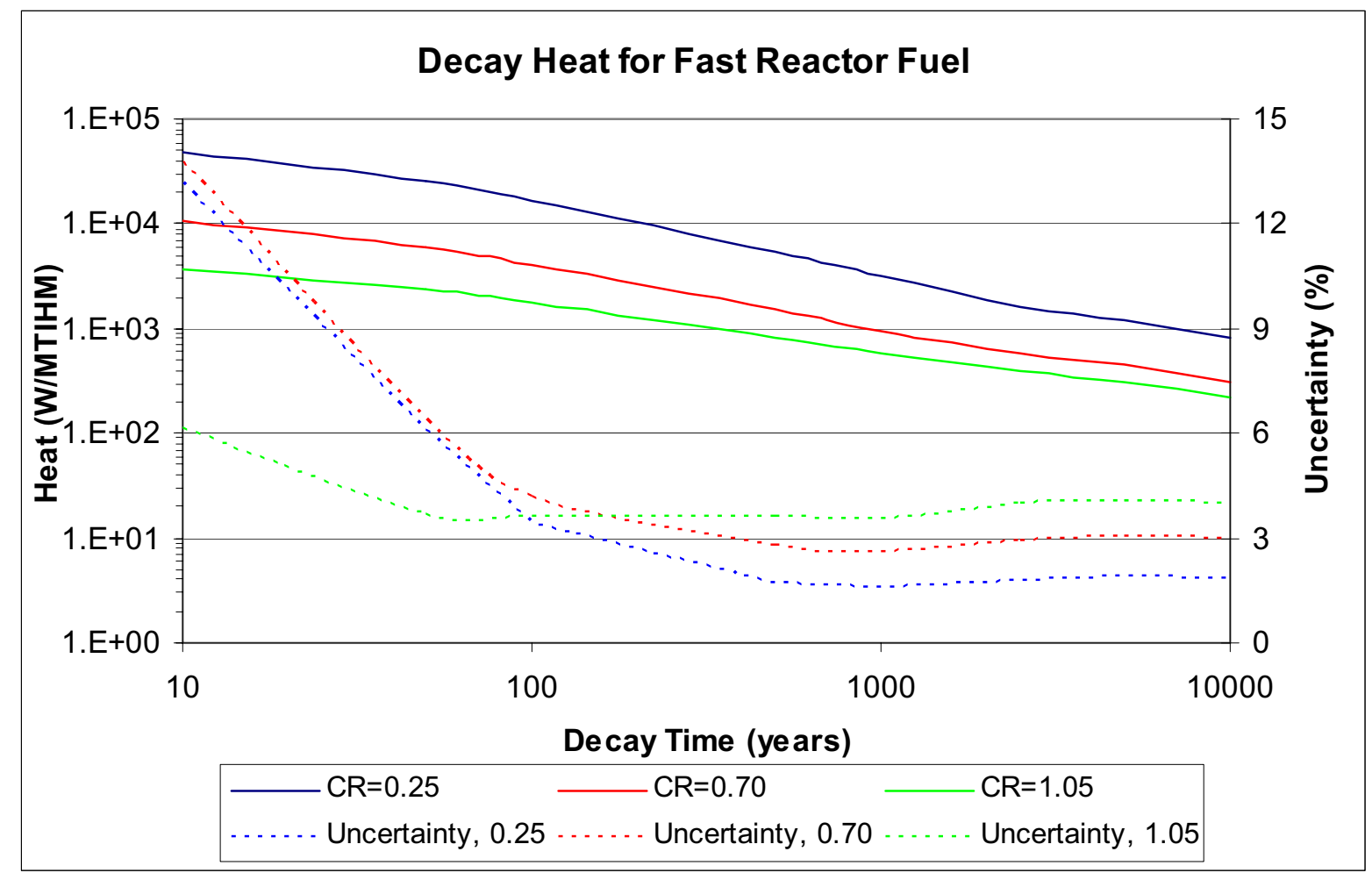

Figure 3.13: Decay heat comparison of three fast reactor fuels.

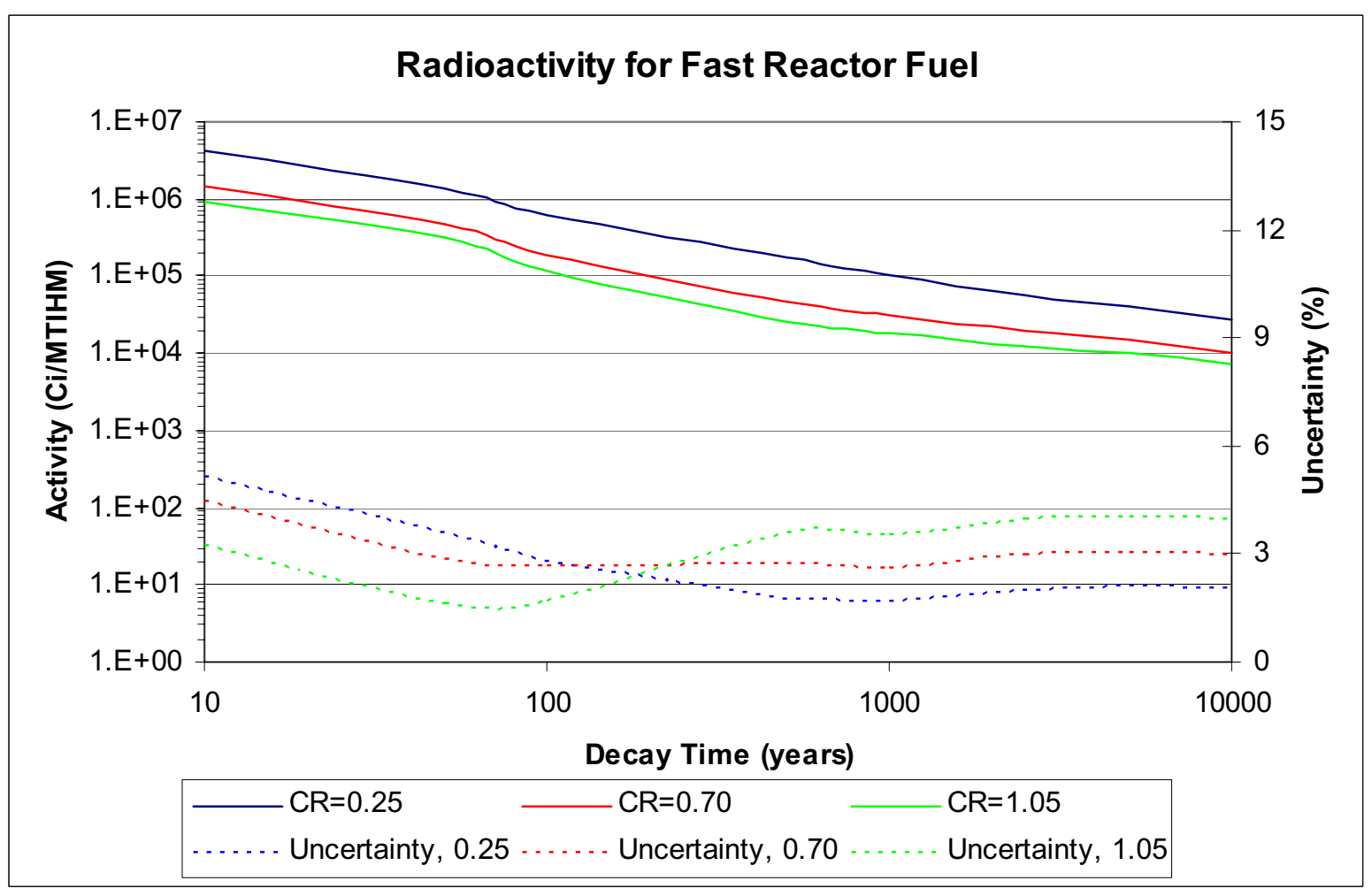

Figure 3.14: Radioactivity comparison for three fast reactor fuels. 


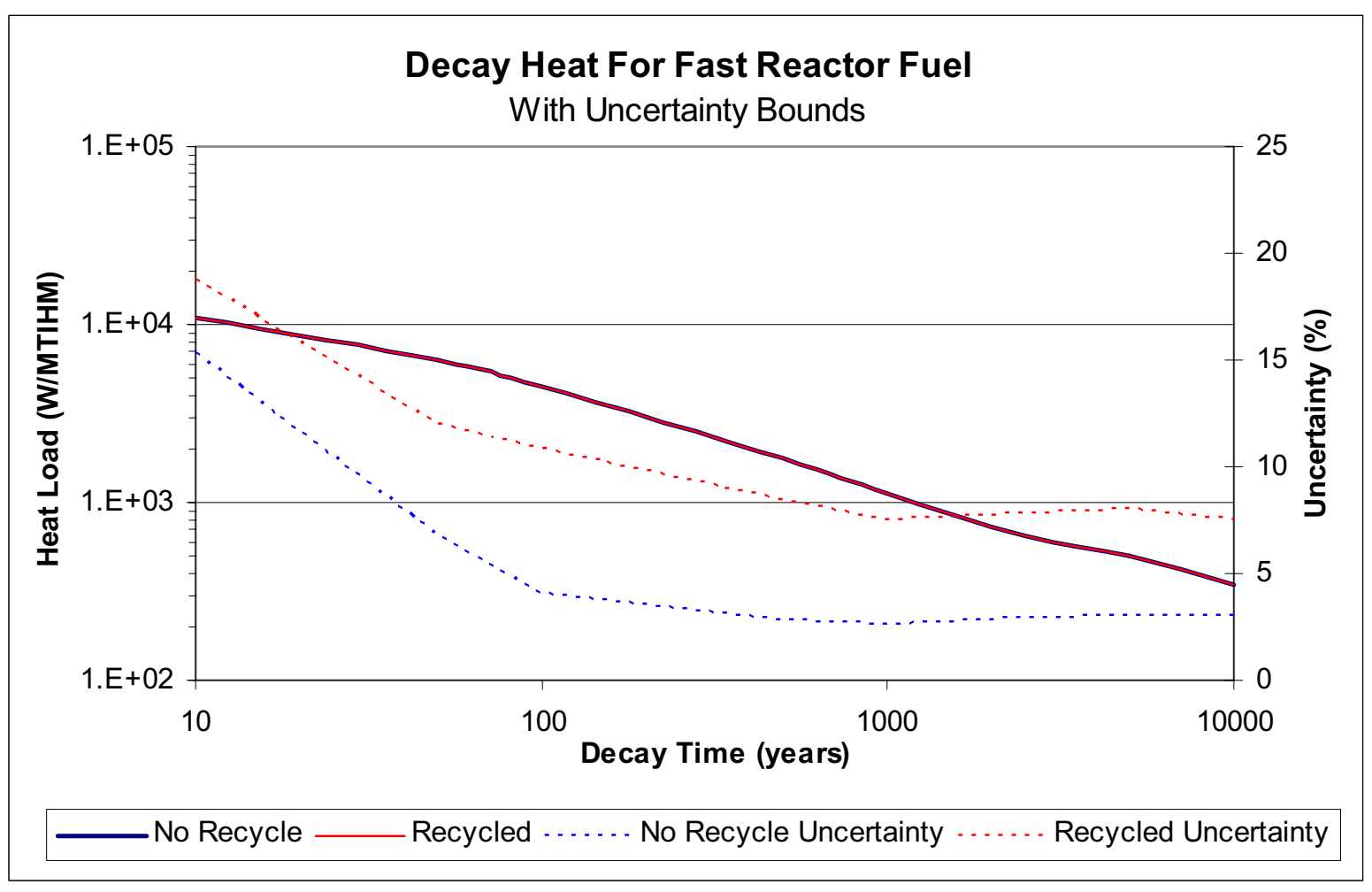

Figure 3.15: Decay heat comparison of once through and recycled fast reactor fuels.

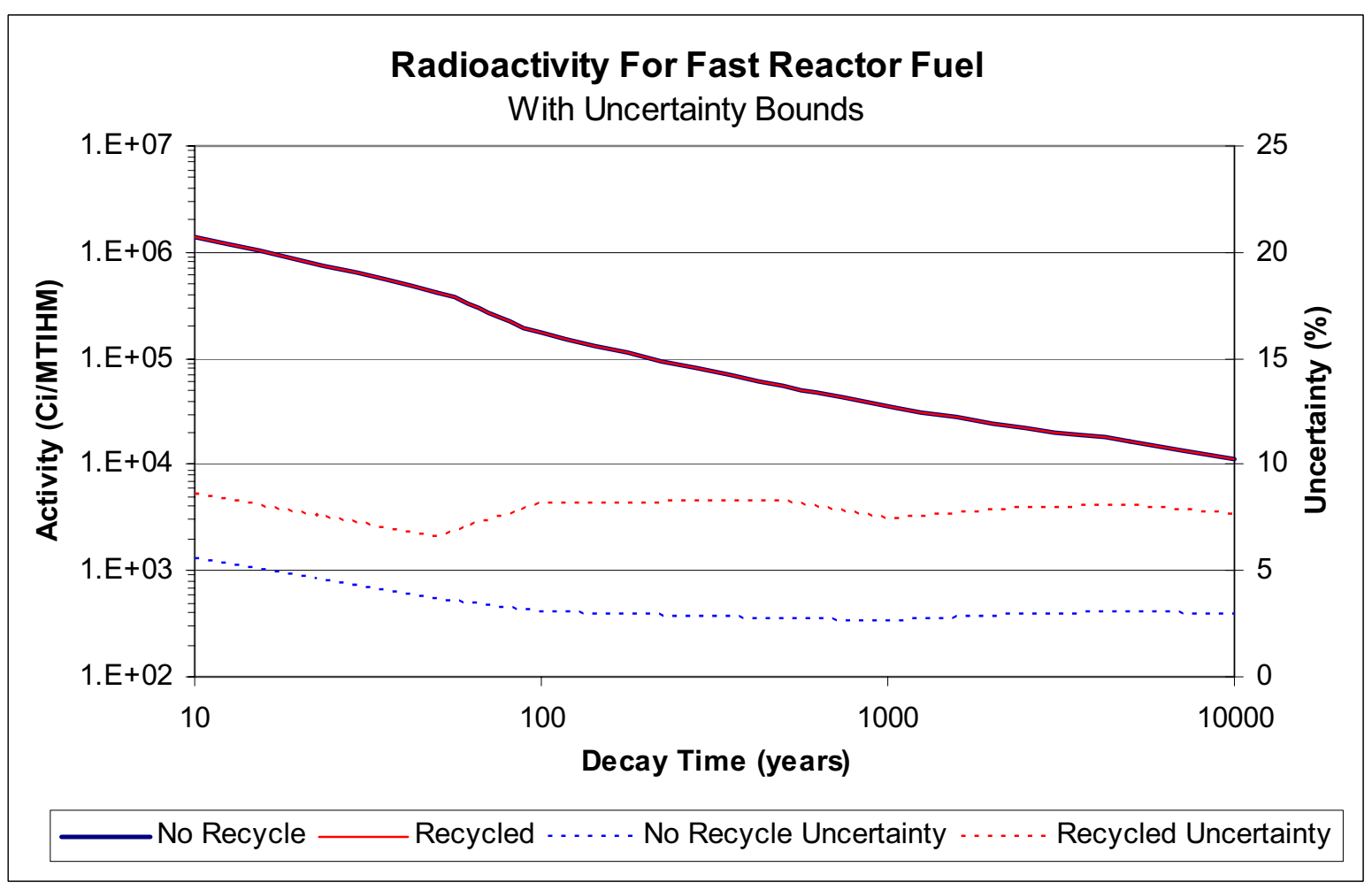

Figure 3.16: Radioactivity comparison of once through and recycled fast reactor fuels. 


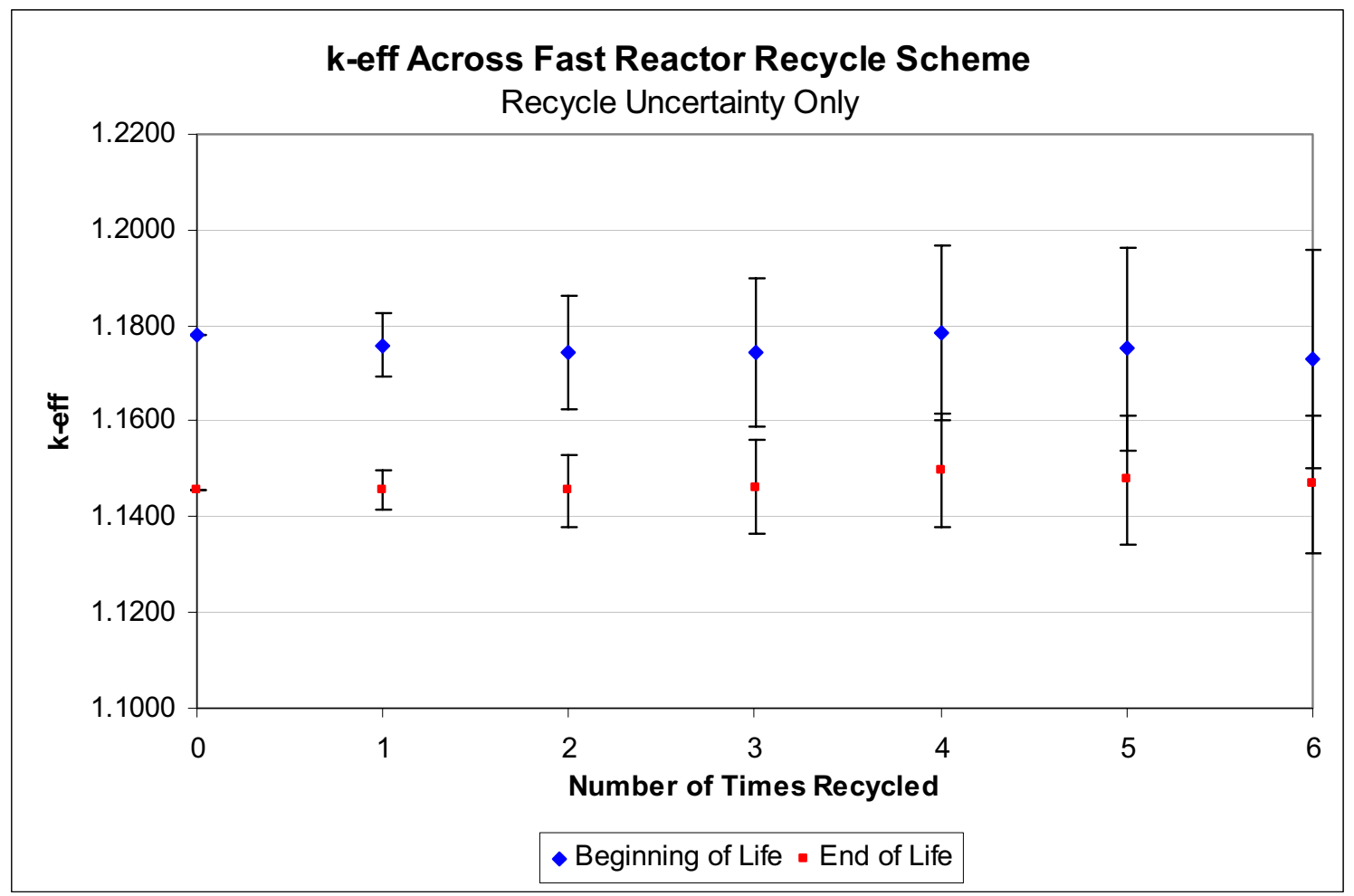

Figure 3.17: k-effective uncertainty due to recycled isotopics uncertainties only.

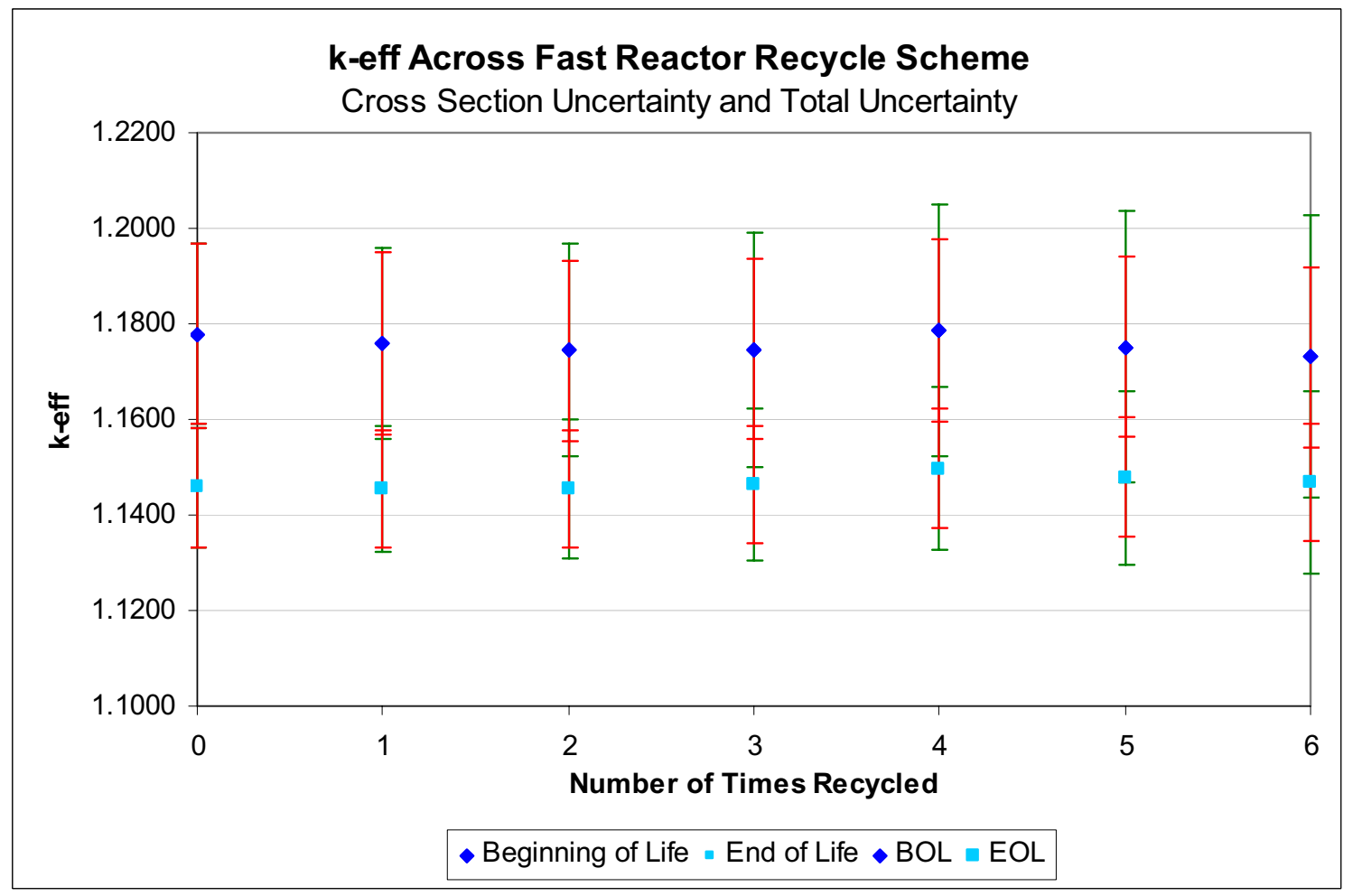

Figure 3.18: k-effective uncertainty due to cross sections and recycled isotopics uncertainties. 


\subsection{REBUS Fast Reactor Equilibrium Model}

With the TRITON sequence producing questionable results for the fast reactor models examined, REBUS was used to examine a fast reactor fuel with uncertainty. The code REBUS, developed by Argonne National Laboratory, is similar to TRITON in that it takes a fuel composition, simulates operating a reactor with that fuel and performs depletion analysis and returns reactor operating parameters (e.g. burnup, k-effective, etc.) REBUS's capability to automatically perform a recycling analysis, brining the fuel to an equilibrium recycling scenario, is exploited here unlike in TRITON where this process was done externally. Further REBUS was able to easily model a $1 / 3$ reactor core with all the heterogeneities as opposed to TRITON's one smeared cell, implying a more reliable model. One draw-back of REBUS is that it can only specifically track the actinide number densities, unlike the SCALE codes which track almost every isotope. For this reason, information from REBUS about amounts of fission and decay products present in the spent fuel is not available. The REBUS k-effective results are closer to expected values than those of the TRITON model, implying a more likely flux spectrum, and thus more likely isotopic composition and fuel depletion. The k-effective for EOC, along with EOC conversion ratio, are provided in Table 3.33 with uncertainty. All results are for the equilibrium composition.

Cross-section uncertainty propagation, using ESM, was implemented in the REBUS code by Dr. Hany Abdel-Khalik. Developed as part of the work reported here was an equilibrium model in REBUS for the fast reactor with a conversion ratio of $\sim 0.77$, using the same recycling specifications given for the recycle scenario using TRITON. The model was executed using Dr. Abdel-Khalik's modified version of REBUS. Table 3.34 gives the discharge isotopics, here EOC core composition normalized to 1 MTHM, and Table 3.35 
presents the isotopic uncertainties. Figure 3.19 - Figure 3.20 show the heat load and radioactivity, respectively, with the uncertainty for the REBUS model compared to the TRITON recycle model. Uncertainties on the LWR recycled isotopics were considered, accomplished by perturbing the LWR isotopics in each input deck via the ESM approach and then determining the uncertainties produced by these, which were very small. The results presented include these perturbations as well as the uncertainty induced by cross section uncertainties. A drawback in REBUS is that correlations between the cross section induced uncertainties and the recycled LWR isotopics induced uncertainties must be assumed to be zero. That is the cross section uncertainties that lead to producing the recycled isotopics uncertainties during LWR operations are not consistently carried forward to the REBUS model of FR operations. To do this, a singlet set of cross sections and their perturbations would need to be employed by the models representing LWR and FR operations. In examining the results, note that these values are much closer to those indicated by the ABTR report [26], in isotopics as well as conversion ratio and k-effective, than are the TRITON results. As discussed before, uncertainties are available only for the actinides and are considerable higher than those predicted by TRITON. With the exception of the initial decay heat uncertainty, the REBUS results had uncertainties more than twice as high as the TRITON model. The higher results are due to a different cross section library, covariance library, and model - all specialized for the fast reactor. The assumptions forced by the resonance treatment in TRITON were recognized to be missing uncertainty components, thus the uncertainties likely underestimated. The REBUS 15-group structure has very little dependence on thermal energies, with 14 of the 15 groups spanning fast and resonance 
energies. Since the structure was designed for the fast reactor, the associated uncertainties are more indicative of the fast system and less restricted by the issues in TRITON.

\begin{tabular}{|l|c|c|}
\hline Operational Parameter & Nominal Value & Uncertainty (\%) \\
\hline EOC k-effective & 0.99925 & 0.2180 \\
\hline EOC Core Conversion Ratio & 0.7695 & 1.7147 \\
\hline
\end{tabular}

Table 3.33: Operating Parameters for REBUS model.

\begin{tabular}{|l|c|l|c|l|c|}
\hline \multicolumn{7}{|c|}{ Discharge Isotopics, grams / MTHM } \\
\hline pb210 & -- & np237 & $3.644 \mathrm{E}+03$ & cm244 & $1.836 \mathrm{E}+03$ \\
\hline ra226 & -- & np239 & -- & cm245 & $4.324 \mathrm{E}+02$ \\
\hline ac227 & -- & pu238 & $6.074 \mathrm{E}+03$ & c 14 & -- \\
\hline th227 & -- & pu239 & $1.030 \mathrm{E}+05$ & se 79 & -- \\
\hline th230 & -- & pu240 & $6.228 \mathrm{E}+04$ & sr 90 & -- \\
\hline pa231 & -- & pu241 & $8.259 \mathrm{E}+03$ & tc 99 & -- \\
\hline u234 & $1.088 \mathrm{E}+02$ & pu242 & $1.230 \mathrm{E}+04$ & i129 & -- \\
\hline u235 & $1.213 \mathrm{E}+03$ & am241 & $7.936 \mathrm{E}+03$ & cs137 & -- \\
\hline u236 & $9.393 \mathrm{E}+01$ & am242m & $5.665 \mathrm{E}+02$ & ba137m & -- \\
\hline u237 & -- & am243 & $3.968 \mathrm{E}+03$ & y90 & -- \\
\hline u238 & $7.878 \mathrm{E}+05$ & cm242 & $2.355 \mathrm{E}+02$ & cs134 & -- \\
\hline
\end{tabular}

Table 3.34: Discharge Isotopics for REBUS model.

\begin{tabular}{|l|c|l|c|l|c|}
\hline \multicolumn{5}{|c|}{ Isotopics Uncertainties, grams / MTHM } \\
\hline pb210 & -- & np237 & 7.344 & cm244 & 20.387 \\
\hline ra226 & -- & np239 & -- & cm245 & 38.508 \\
\hline ac227 & -- & pu238 & 18.616 & c 14 & -- \\
\hline th227 & -- & pu239 & 1.336 & se 79 & -- \\
\hline th230 & -- & pu240 & 7.463 & sr 90 & -- \\
\hline pa231 & -- & pu241 & 10.570 & tc 99 & -- \\
\hline u234 & 18.383 & pu242 & 18.769 & i129 & -- \\
\hline u235 & 0.809 & am241 & 10.163 & cs137 & -- \\
\hline u236 & 2.034 & am242m & 14.836 & ba137m & -- \\
\hline u237 & -- & am243 & 17.986 & y90 & -- \\
\hline u238 & 0.950 & cm242 & 8.856 & cs134 & -- \\
\hline
\end{tabular}

Table 3.35: Isotopc Uncertainties for REBUS model. 


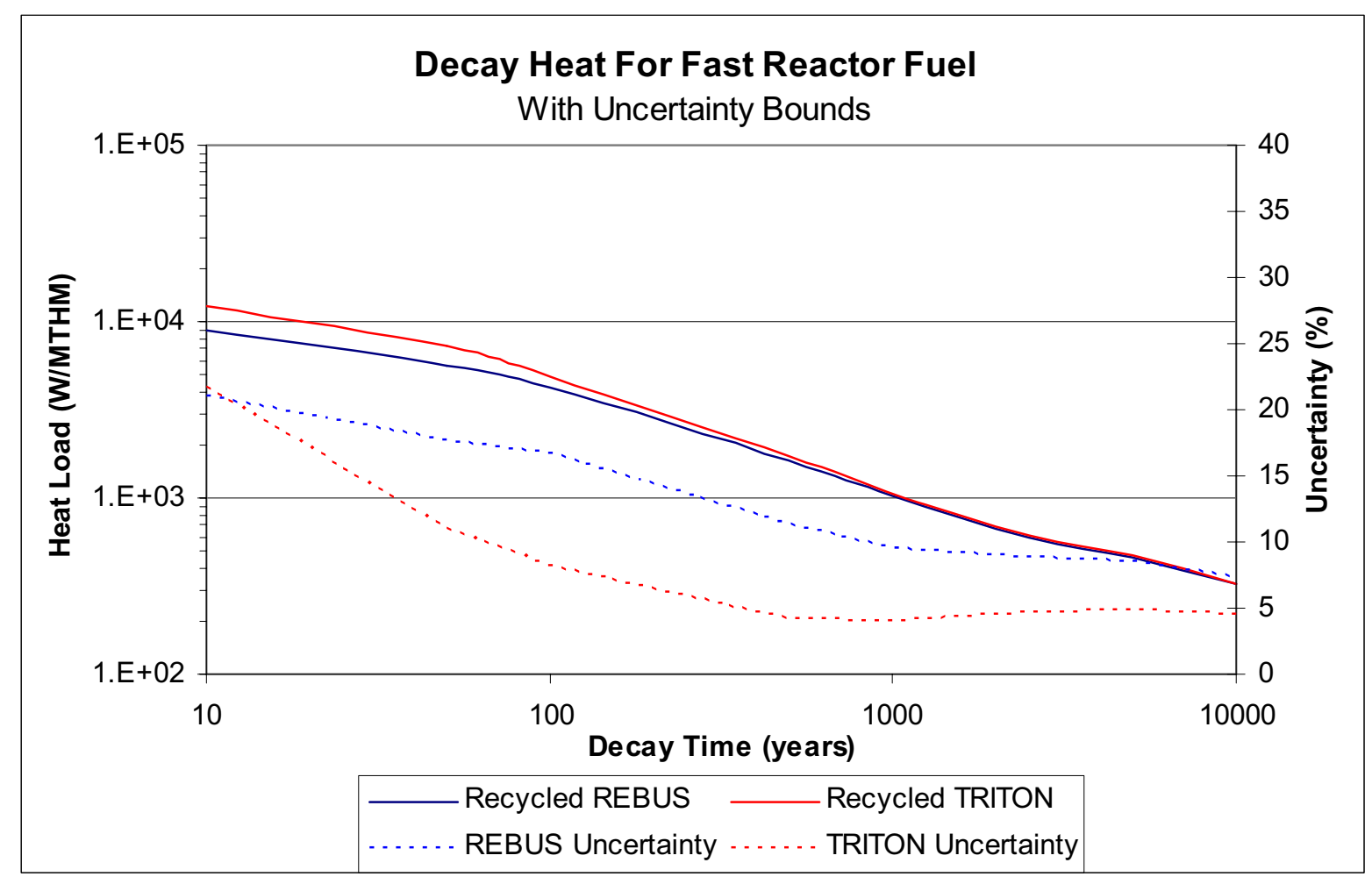

Figure 3.19: Decay heat comparison of REBUS and TRITON Models.

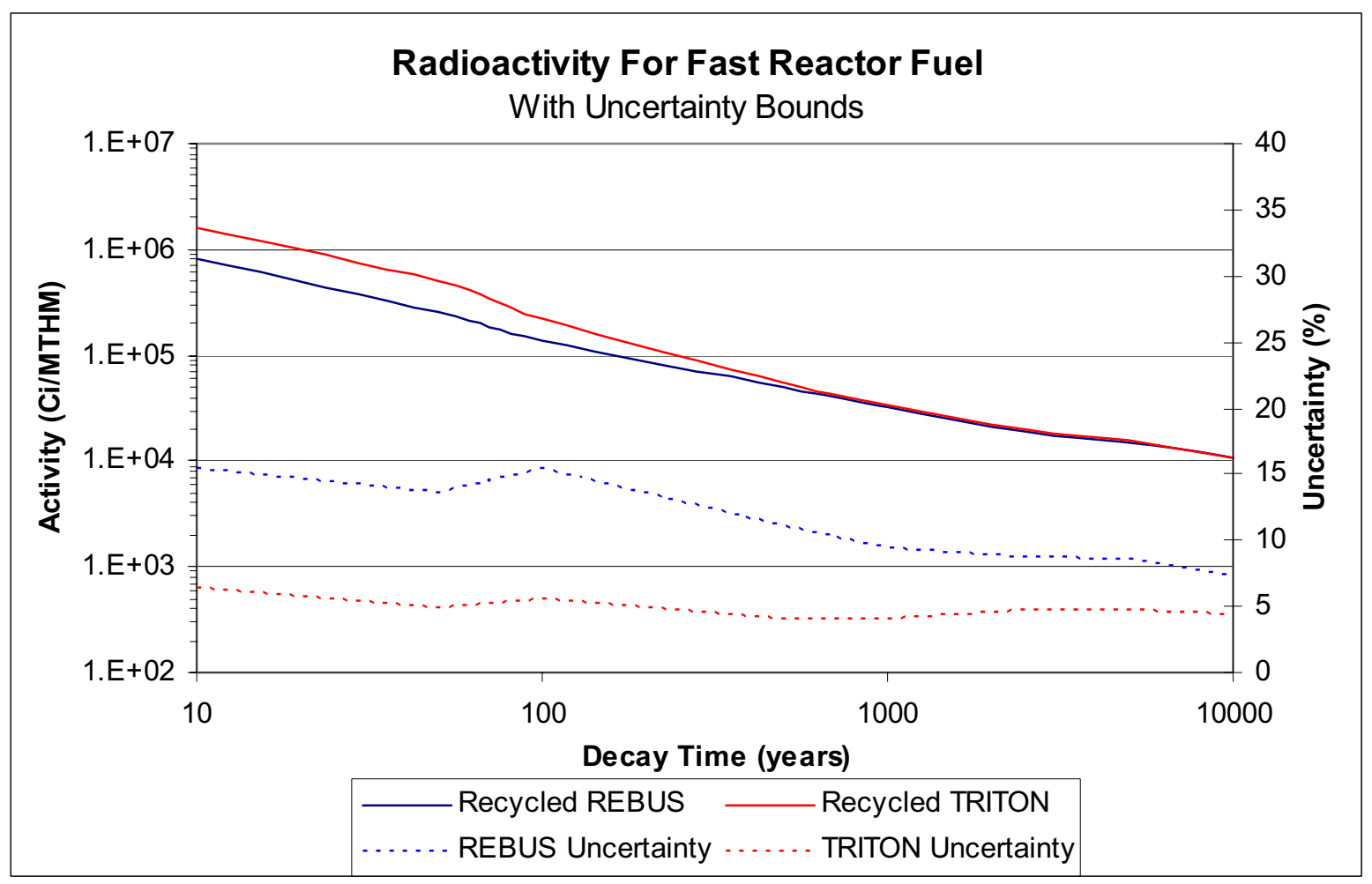

Figure 3.20: Radioactivity comparison of REBUS and TRITON Models. 


\section{Discussion and Conclusions}

\subsection{Discussion of the Use of ESM in this Study}

The Efficient Subspace Method (ESM) has been demonstrated to produce results equivalent to those of traditional stochastic sampling methods. While this provides an alternative to these methods in any case where stochastic sampling could be used, it is most beneficial in models where stochastic methods would not be practical. For the case of the TRITON and REBUS models, where perturbed input data numbered in thousands, stochastic sampling would have taken at least twice as long as using ESM. In most multi-scale, multiphysics codes, such as the TRITON sequence in SCALE, input data do number in the thousands, if not orders of magnitude higher. For example, the core simulator FORMOSA developed at North Carolina State University has millions of input data, and, if perturbed, would have thousands of millions of perturbations. With growing reliance on computer simulation in many industries, including nuclear, these large, complex models are becoming more popular and necessary. Thus, having a method to quickly and efficiently propagate uncertainties becomes a much desired capability. Since one goal of this study was to demonstrate the capability of ESM to perform in this manner, it can be concluded that ESM can be successfully used on large, complex models while producing results that are equivalent to traditional sampling methods.

\subsection{Discussion Concerning the Results of the Models}

The other objective of this study was to determine how cross section uncertainties affect back-end fuel cycle metrics such as decay heat, radioactivity, and radiotoxicity. Essentially, this implies determining how cross section uncertainty affects the composition of 
what is discharged from the reactor, since all other metrics are directly proportional to isotopic masses. For the UOX fuels, in both the simplified and detailed models, uncertainty on these metrics was 1-2 \% for the first 100 years and then 3-5\% thereafter. Short term uncertainty is dominated by low-uncertainty fission products that make up the majority of the heat load and activity in the first 100 years. The constant uncertainty in the long term is due to a few long lived actinides, mainly plutonium and americium isotopes. When looking at the simplified MOX fuels, the uncertainty increases in the short term, especially for the decay heat, due to uncertainty in fission products and short lived actinides caused by the uncertainties on the fission and absorption cross sections of the minor actinides, which are now present in greater quantities. However, the long term uncertainty is still about $5 \%$, stemming from long lived actinides with similar uncertainties to those for the same isotopes in the UOX fuel.

Considerable differences between the short term uncertainties for the PWR models were discovered between the simplified ORIGEN model and the TRITON model. An evaluation concluded this was the result of the resonance treatment applied in the TRITON sequence. In the simple ORIGEN models, prepared 1-group cross sections were perturbed just before the depletion calculation. However in TRITON, only the reference 44-group cross sections were perturbed, and the resonance component in the resolved resonance regions missed, which diminished the affect of the perturbations. Further study on this topic was beyond the scope of the current work.

The fast reactor TRITON models, though the short-comings of the model are recognized, showed considerably higher values for heat load and radioactivity than those of the PWR model. This was expected because of the nature of the fuel, that being a large 
weight percent of transuranics which contribute to the longevity and magnitude of these metrics. The increasing activity and decay heat with respect to decreasing conversion ratio is the result of increasing the transuranic enrichment and extending the burnup to reach the clad fluence limit, therefore building up more of products contributing to these metrics. The increasing uncertainty with increasing conversion ratio results from the increase of uranium content and fissile fraction of the TRU, which have large, highly correlated uncertainties. Still the over all long term uncertainty of these fuels is about 3-5\%.

The TRITON recycling models show the expected result of increasing uncertainty as fuel is recycled to an equilibrium. While this process nearly doubles the uncertainty, long term uncertainties are still on the order of $5 \%$ but short term uncertainties could increase from 10 to $20 \%$, which would result in more conservative handling and processing in the near term. The main concern of recycling would appear to be the uncertain compositions being used as fuel for the reactor, but this is easily eliminated by measuring the isotopic masses prior to refabrication. Recycling isotopics uncertainties add a non-negligible amount to the already present cross-section uncertainty on k-effective values.

The REBUS equilibrium recycling model was nominally comparable to the TRITON model in terms of long term heat load and radioactivity. However the nominal REBUS results are regarded as more indicative of the properties of the spent fuel in consideration, since that model very nearly matched the one in the Argonne report after which it was designed, both in isotopics and operating conditions. The uncertainty displayed by the REBUS model was, however, considerable different than that of the TRITON model, in some instances up to 7 times greater. This indicates that, as stated in the discussion on resonance treatment, that the TRITON model was underestimating the uncertainty on the 
isotopics, which propagated to the metrics examined. Also the $11 \%$ uncertainty on the conversion ratio implies that reactor operation may or may not destroy the desired amount of actinides, which is essentially the job of this type of reactor. So, for this equilibrium fuel cycle, judging from the REBUS results, the fuel that is discharged from the reactor needs to be carefully measured in terms of isotopics before refabrication or would have to be treated very conservatively if immediately disposed of. Thankfully there would not be much of it to dispose of if widespread use of fast reactors destroyed much of the spent LWR fuel.

If one is simply disposing of UOX or even MOX fuel that was burned in a LWR, uncertainties due to cross sections seem to be of little concern. This is especially true when one considers the highly restrictive geological uncertainties, waste package material uncertainties, and even reactor operation uncertainties, which can easily overwhelm the low uncertainties seen for these models. However, when one considers reprocessing, crosssection uncertainties become more important. High short term uncertainties on decay heat and activity would result in more conservative handling of fuels for reprocessing. Isotopic uncertainties for fuel mainly impact composition changes during irradiation and design of the repository, since isotopics can be measured prior to refabrication. The decrease in repository loading due to fast reactor operation likely will outweigh the uncertainty associated with that fuel, but further study on such margins is needed. Concluding from these results, cross section uncertainty would need to be reduced to better the operation of fast reactors and dispose of their reprocessed fuel.

\subsection{Recommendations and Future Work}

The immediate future work is to develop the uncertainty propagation methodology in this work into a model that is usable within SINEMA's primary software, GENIUS. In 
keeping with the GENIUS format, this will be a series of tables from which to interpolate data concerning uncertainties, given a fuel composition and discharge burnup, for each reactor type. Further development should be implemented in the TRITON sequence to improve the treatment of resonance uncertainties.

Beyond the cross section uncertainties propagated into the isotopics, which is all that was considered in this work, reprocessing includes chemical reaction rates and uncertainties, plant efficiencies, separation time, storage and transport time, etc. All of these can play a role in the composition of the fuel that is fabricated. Also, as shown in a very simple experiment for an LWR, operational uncertainties can add much more to the uncertainties seen in the discharged isotopics than the cross sections do, so they warrant further study. Finally, the metrics considered herein were rather simplistic metrics, directly related to isotopics masses and instantaneous in nature. The uncertainty margins on more thorough, integral metrics (e.g. long-term heat integral, or dose calculations) would also be of interest, especially for disposal purposes. For the recycling cases, further study should be conducted to include correlations between recycled isotopics uncertainties and cross sections uncertainties, which were assumed uncorrelated in this work. Finally the cut-off value for the choice of samples to run could be adjusted, as the value used in this work was a very conservative. Removing unused cross sections and working with absolute values rather than relative values could help the user to better select which samples to run. In this work, an unimportant cross section may have a high relative uncertainty and that sample would be kept when it does not actually need to be run. 


\section{References}

[1] Official Site of the Simulation Institute for Nuclear Energy Analysis. 2006-2007. 1 June 2006. <http://thesinema.org>.

[2] Wigeland, R., et. al. "Repository Impact of LWR MOX and Fast Reactor Recycling Options". ANS Topical Meeting. New Orleans, LA 2003.

[3] Yacout, A., et. al. "Dynamic Analysis of the AFCI Scenarios". Proceedings of the PHYSOR 2004: The Physics of Fuel Cycles and Advanced Nuclear Systems - Global Developments 2004: 785-794

[4] Bathke, C., et. al. "Report of LANL Advanced Fuel Cycle Systems Analyses for FY 2003". Los Alamos National Laboratory. 2004.

[5] J. Saling. Radioactive Waste Management, $2^{\text {nd }}$ Edition. Taylor and Francis, 2001.

[6] Bunn, M., et. al. "Economics of Reprocessing vs. Direct Disposal of Spent Nuclear Fuel". Harvard University, 2003

[7] Rose, P. F. and C. L. Dunford. "ENDF-102: Data Formats and Procedures for the Evaluated Nuclear Data File ENDF-6". Brookhaven National Laboratory. 1990.

[8] Turinsky, P., H. Abdel-Khalik, and T.E. Stover. "Development of Uncertainty Analysis Capabilities for the SINEMA Project, FY 2006 Report". North Carolina State University. 2006.

[9] Duderstadt, J., and L. Hamilton. Nuclear Reactor Analysis. Hoboken, NJ. John Wiley \& Sons, Inc., 1976.

[10] Schneider, E., and C. Bathke, "Sensitivity of Fuel Cycle Performance Metrics to Perturbations in Cross-Sections". Transactions of the American Nuclear Society v 92, 2005.

[11] Ronen, Yigal. Uncertainty Analysis. Boca Raton, FL. CRC Press Inc., 1988.

[12] Aliberti, G., et. al. "Nuclear data sensitivity, uncertainty and target accuracy assessment for future nuclear systems". Annals of Nuclear Energy, 33(8), 2006: 700-733.

[13] Aliberti, G., et al. "Impact of Nuclear Data Uncertainties on Transmutation of Actinides in Accelerator-Driven Assemblies," Nuclear Science and Engineering v 146, 2004: 1350.

[14] H. Abdel-Khalik, "Adaptive Core Simulation". Ph.D. Dissertation. North Carolina State University, 2004.

[15] Cacuci, Dan. Sensitivity and Uncertainty Analysis, Volume I. Chapman \& Hall/CRC Press, 2005.

[16] Olsson, A., and G. Sandberg, "Latin Hypercube Sampling for Stochastic Finite Element Analysis". Journal of Engineering Mechanics January 2002.

[17] Bendat, J., and A. Piersol. Random Data: Analysis and Measurement Procedures. $2^{\text {nd }}$ Ed. Hoboken, NJ. John Wiley \& Sons, Inc., 1986. 
[18] Kawano, T., et. al. "Evaluations and Propagation of the Pu-239 Fission Cross-Section Uncertainties Using a Monte Carlo Technilque". Nuclear Science and Engineering v 153, n 1, May 2006: 1-7.

[19] Oak Ridge National Laboratory. SCALE: A Modular Code System for Performing Standardized Computer Analyses for Licensing Evaluations. ORNL/TM-2005/39, Version 5, Vols. I-III, 2005.

[20] Argonne National Laboratory. REBUS-3/Variant 8.0: Code System for Analysis of Fast Reactor Fuel Cycles. CCC-653, REBUS-3, Variant 8.0, 2001.

[21] Gauld, I. C., et. al. "ORIGEN-S: Scale System Module to Calculate Fuel Depletion, Actinide Transmutation, Fission Product Buildup and Decay, and Associated Radiation Source Terms". Oak Ridge National Laboratory. ORNL/NUREG/CSD-2/V2/R7, 2004

[22] Gauld, I.C., and O. Hermann "SAS2: A Coupled One-Dimensional Depletion and Shielding Analysis Module". Oak Ridge National Laboratory. ORNL/TM-2005/39 Version 5 Vol. I, Book 3, Sect. S2, 2005.

[23] DeHart, M.D. “TRITON: A Two-Dimensional Depletion Sequence for Characterization of Spent Nuclear Fuel". Oak Ridge National Laboratory. ORNL/TM-2005/39 Version 5 Vol. I, Book 3, Sect. T1, 2005.

[24] Rochman, D., et. al. "Preliminary Cross Section and v-bar Covariances for WPEC Subgroup 26". National Nuclear Data Center. Brookhaven National Laboratory. BNL77407-2007-IR, 2007

[25] Argonne National Laboratory. $\mathrm{MC}^{2}-2$ : Code System for Calculating Fast Neutron Spectra and Multigroup Cross-Sections. PSR-350, MC ${ }^{2}-2,2000$.

[26] Chang, Y.I., et. al. “Advanced Burner Test Reactor, Preconceptual Design Report”. Argonne National Laboratory. ANL-AFCI-173, 2006.

[27] Tarantola, A., Inverse Problem Theory: Methods for Data Fitting and Model Parameter Estimation. Elsevier Science Publishers B. V., 1987.

[28] Figliola, F., and D. Beasley. Theory and Design for Mechanical Measurements. $3^{\text {rd }}$ Ed. Hoboken, NJ. John Wiley \& Sons, Inc., 2000.

[29] Tong, Y.L. Multivariate Normal Distribution. New York, NY. Springer-Verlag, 1990.

[30] Jordan, W., et. al. "SCALE Cross-Section Libraries". Oak Ridge National Laboratory. ORNL/TM-2005/39 Version 5 Vol. III, Sect. M4, 2005.

[31] Rearden, B. "Sensitivity Utility Modules". Oak Ridge National Laboratory. ORNL/TM2005/39 Version 5 Vol. III, Sect. M18, 2005.

[32] Hermann, O., and M. DeHart. "Validation of SCALE (SAS2H) Isotopic Predictions for BWR Spent Fuel”. Oak Ridge National Laboratory. ORNL/TM-13315, 1998.

[33] Cochran, R., and N. Tsoulfanidis. Nuclear Fuel Cycle: Analysis and Management. $2^{\text {nd }}$ Ed. Washington D.C. American Nuclear Society, 2002. 
(This page intentionally left blank.) 


\section{Appendix A: Fuel Models}

\section{The Typical LWR Model Using a UOX Fuel}

For use in base cases and general examples, SCALE is distributed with a flux spectrum and three-group card image cross-section library that are representative of a typical LWR. The first sampling routine was implemented with this resource. The input library can be used in its three-group form along with the flux spectrum supplied directly to ORIGEN or the library may be collapsed to a one-group binary library. This model was taken directly from the ORIGEN users manual and changed only in the enrichment of U-235 to 4.5 w/o rather than the $3.3 \mathrm{w} / \mathrm{o}$ of the original example problem so the reader is referred to the ORIGEN users manual [21].

\section{The PWR Model Using a UOX Fuel}

The PWR model used in this study is based on the example provided with the SCALE 5.0 SAS2H User's Manual, that being a Westinghouse type PWR fuel assembly (Figure A.II.1). The assembly is 17 pins by 17 pins with 25 water holes and an active fuel length of 12 feet $(365.76 \mathrm{~cm})$ in a square pitch design. Fuel rods have a pitch of $1.25984 \mathrm{~cm}$ and an outside diameter of $0.83566 \mathrm{~cm}$ (no gap in this model). The fuel itself is $\mathrm{UO}_{2}$ containing $461.4 \mathrm{~kg}$ of uranium in the proportions $4.50 \mathrm{w} / \mathrm{o} \mathrm{U-235,} 95.472 \mathrm{w} / \mathrm{o} \mathrm{U}-238$ and $0.028 \mathrm{w} / \mathrm{o} \mathrm{U}-$ 234 and is volume fraction weighted $(\mathrm{VF}=0.90182)$ for the given fuel assembly based on the volume weighting method described in the SAS2H user's manual, i.e. VF depends on mass of fuel and volume of fuel assembly. Cladding is zirc2 (versus actual zirc4) and the moderator is water. Operating temperatures are 811, 570 and 570 degrees Fahrenheit for the fuel, clad and moderator respectively with the moderator density at $0.733 \mathrm{~g} / \mathrm{cm}^{3}$. The 
SAS2H model is burned at a specific power of $18.456 \mathrm{MW} /$ assembly for 1000 days giving a total burnup of $40 \mathrm{GWD} / \mathrm{MTU}$.

The corresponding ORIGEN model (Figure A.II.2), from which sampling is conducted, is one metric ton of the $4.5 \mathrm{w} / \mathrm{o}$ UOX fuel with approximately $271 \mathrm{~kg}$ of zirc2 cladding. The ORIGEN model is set to deplete the fuel using the binary cross section library generated from the fuel specific SAS2H model. Note: cross section perturbations are introduced directly into this binary working library during the sampling procedure. Fuel specific power density is $40 \mathrm{MW} / \mathrm{MTU}$ for a cycle length of 1000 days giving the same 40GWD/MTU burnup as the SAS2H Model. The ORIGEN model is set to "burn" the fuel for 10 equal time steps at $100 \%$ power and then print out decay isotopics at charge, discharge, 1 , $5,10,50,100,500,1000,2500,5000$, and 10000 year times. ORIGEN also has the availability to print the decay heat, activity, and radiotoxicity for these times.

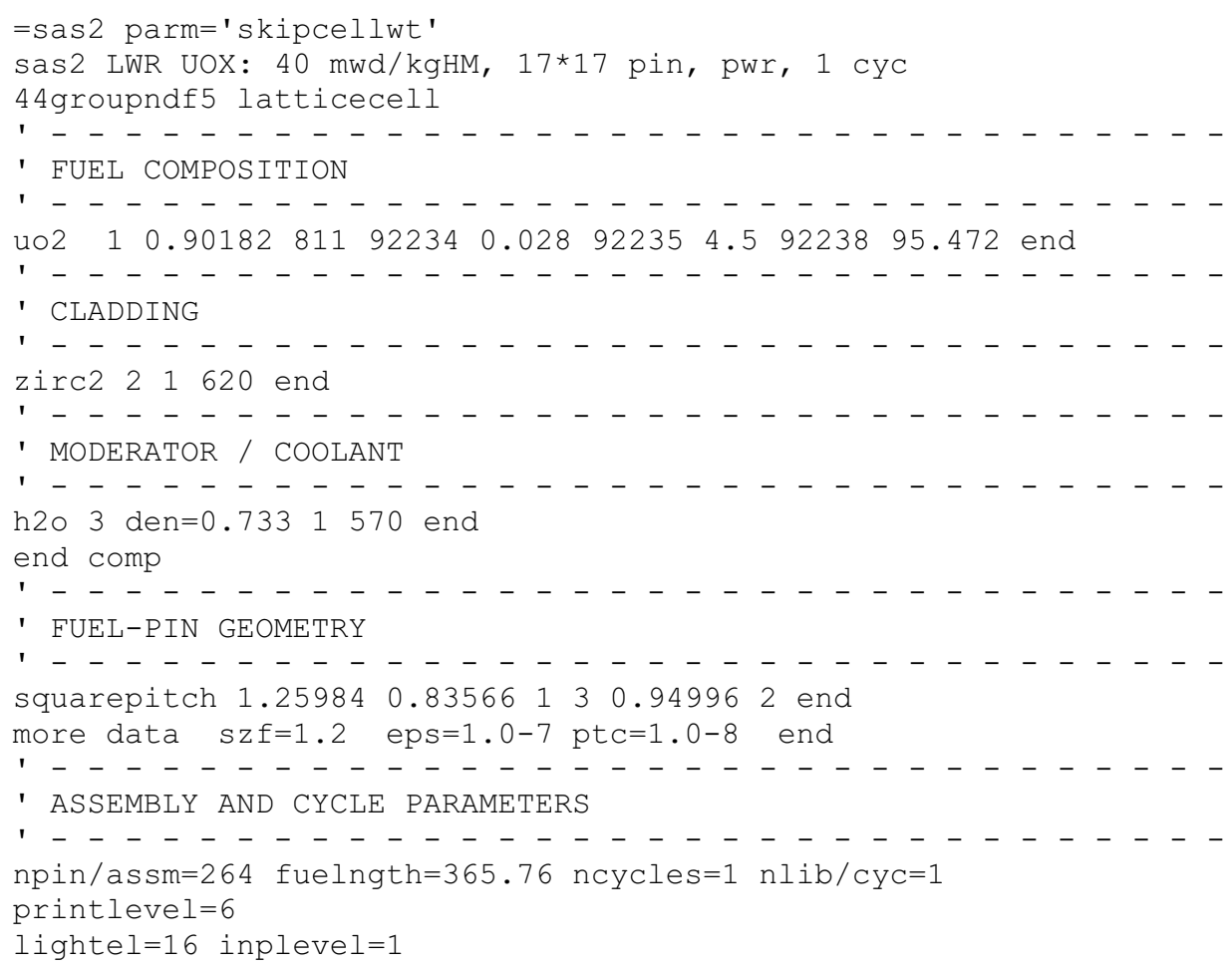

Figure A.II.1: SAS2H Model, PWR, 4.5 w/o and 40 GWD/MTU 
numins $=1$ ortube $=0.61214$ srtube $=0.5715$ facmesh $=1.4$ end power=17.3025 burn=1066.67 down=0 end 'power=18.456 burn=1000.0 down=0 end

Figure A.II.1: SAS2H Model, PWR, 4.5 w/o and 40 GWD/MTU, cont.

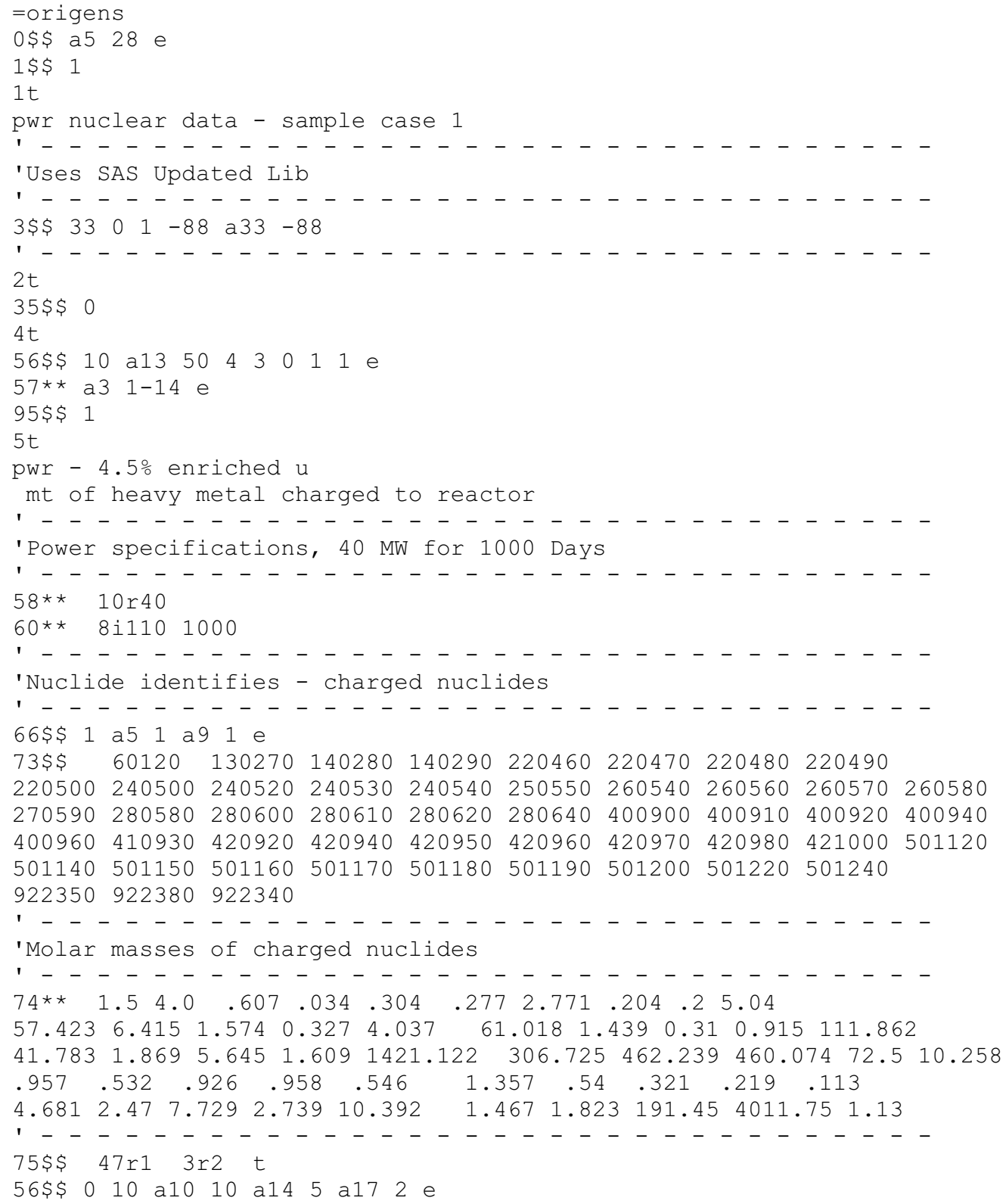

Figure A.II.2: ORIGEN Model, PWR, 4.5 w/o and 40 GWD/MTU 


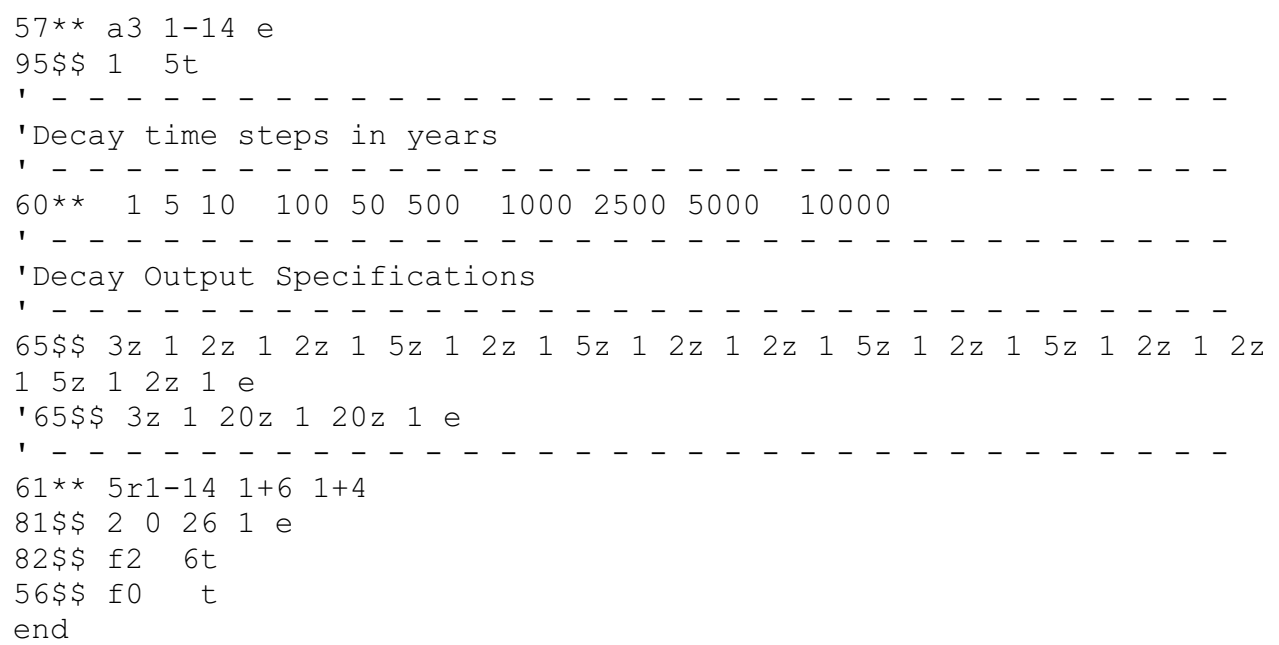

Figure A.II.2: ORIGEN Model, PWR, 4.5 w/o and 40 GWD/MTU, cont.

\section{The BWR Model Using a UOX Fuel}

The BWR model used in this study is adapted from a whole assembly SAS2H model in an Oak Ridge National Lab report concerning validation of SAS2H for BWR predictions (Figure A.III.1). The assembly is a General Electric type 7x7 fuel assembly with an active fuel length of 12.17 feet $(370.84 \mathrm{~cm})$ in a square pitch design. Fuel rods have a pitch of $1.875 \mathrm{~cm}$ and an outside diameter of $1.242 \mathrm{~cm}$ (no gap in this model). The model has been simplified from the mentioned report in that it has been homogenized (no burnable poisons or water holes) and has an initial enrichment of $4.5 \mathrm{w} / \mathrm{o}$ so as to match the PWR fuel in that regard. The fuel itself is $\mathrm{UO}_{2}$ containing $190.71 \mathrm{~kg}$ of uranium in the proportions $4.50 \mathrm{w} / \mathrm{o}$ $\mathrm{U}-235,95.472 \mathrm{w} / \mathrm{o} \mathrm{U}-238$ and $0.028 \mathrm{w} / \mathrm{o} \mathrm{U}-234$ and is volume fraction weighted $(\mathrm{VF}=0.5589)$ for the given fuel assembly based on the volume weighting method described in the SAS2H user's manual, i.e. VF depends on mass of fuel and volume of fuel assembly. Cladding is zirc 2 and the moderator is water. Average operating temperatures are 840,620 and 558 degrees Fahrenheit for the fuel, clad and moderator respectively. Void fraction for the whole assembly model is handled by choosing an average moderator density based on the 
void fraction: $\rho_{\text {average }}=(\alpha) \rho_{\text {vapor }}+(1-\alpha) \rho_{\text {liquid. }}$. The saturated liquid and vapor densities of water at 558 degrees Fahrenheit are 0.74178 and $0.03593 \mathrm{~g} / \mathrm{cm}^{3}$ respectively and the void fractions used in this study were $0,0.35,0.50$ and 0.65 . The SAS2H model is burned at a specific power of $7.628 \mathrm{MW} /$ assembly for 1000 days giving a total burnup of 40 GWD/MTU.

The corresponding ORIGEN model (Figure A.III.2), from which sampling is conducted, is one metric ton of the $4.5 \mathrm{w} / \mathrm{o}$ UOX fuel with approximately $271 \mathrm{~kg}$ of zirc2 cladding. The ORIGEN model is set to deplete the fuel using the binary cross section library generated from the fuel specific SAS2H model. Note: cross section perturbations are introduced directly into this binary working library during the sampling procedure. Fuel specific power density is $40 \mathrm{MW} / \mathrm{MTU}$ for a cycle length of 1000 days giving the same 40GWD/MTU burnup as the SAS2H Model. The ORIGEN model is set to "burn" the fuel for 10 equal time steps at $100 \%$ power and then print out decay isotopics at charge, discharge, 1 , $5,10,50,100,500,1000,2500,5000$, and 10000 year times. ORIGEN also has the availability to print the decay heat, activity, and radiotoxicity for these times.

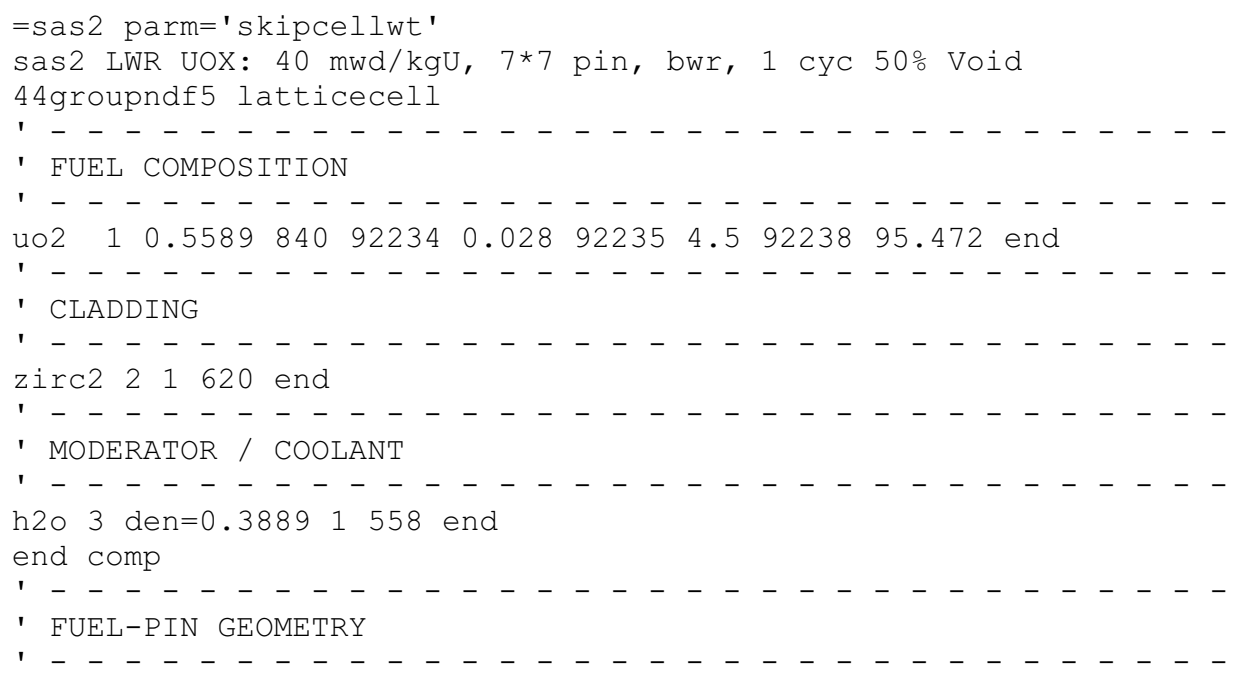

Figure A.III.1: SAS2H Model, BWR, 4.5 w/o and 40 GWD/MTU 


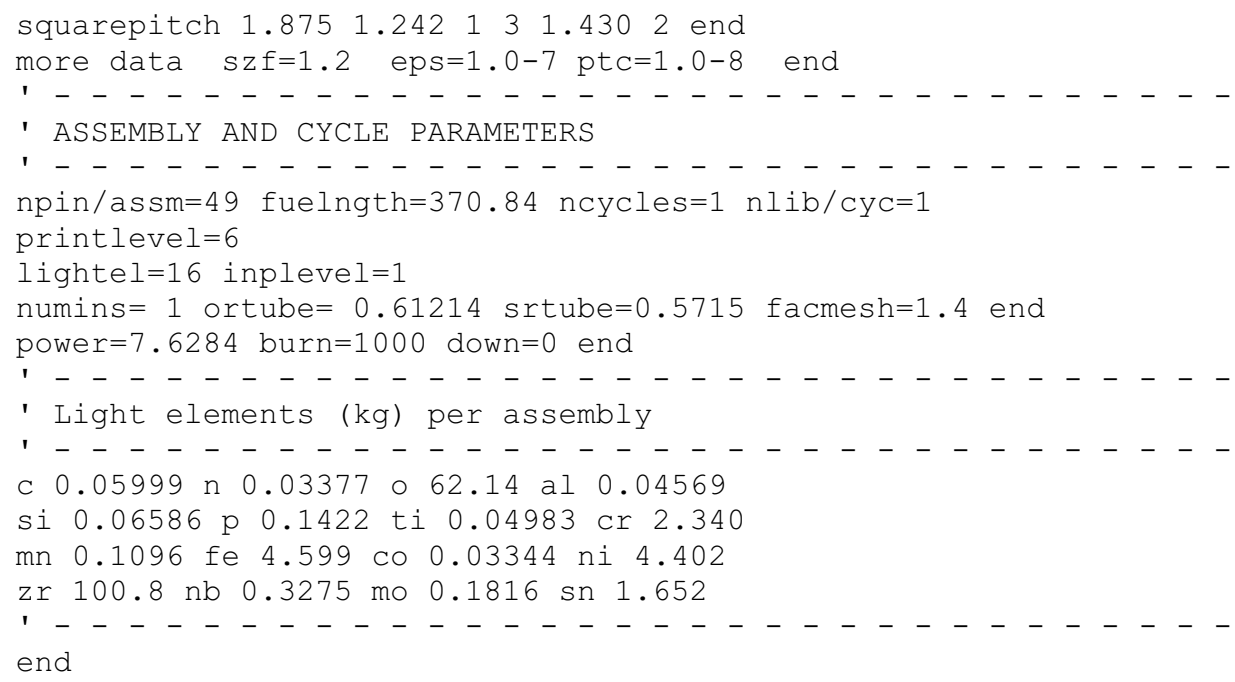

Figure A.III.1: SAS2H Model, BWR, 4.5 w/o and 40 GWD/MTU, cont.

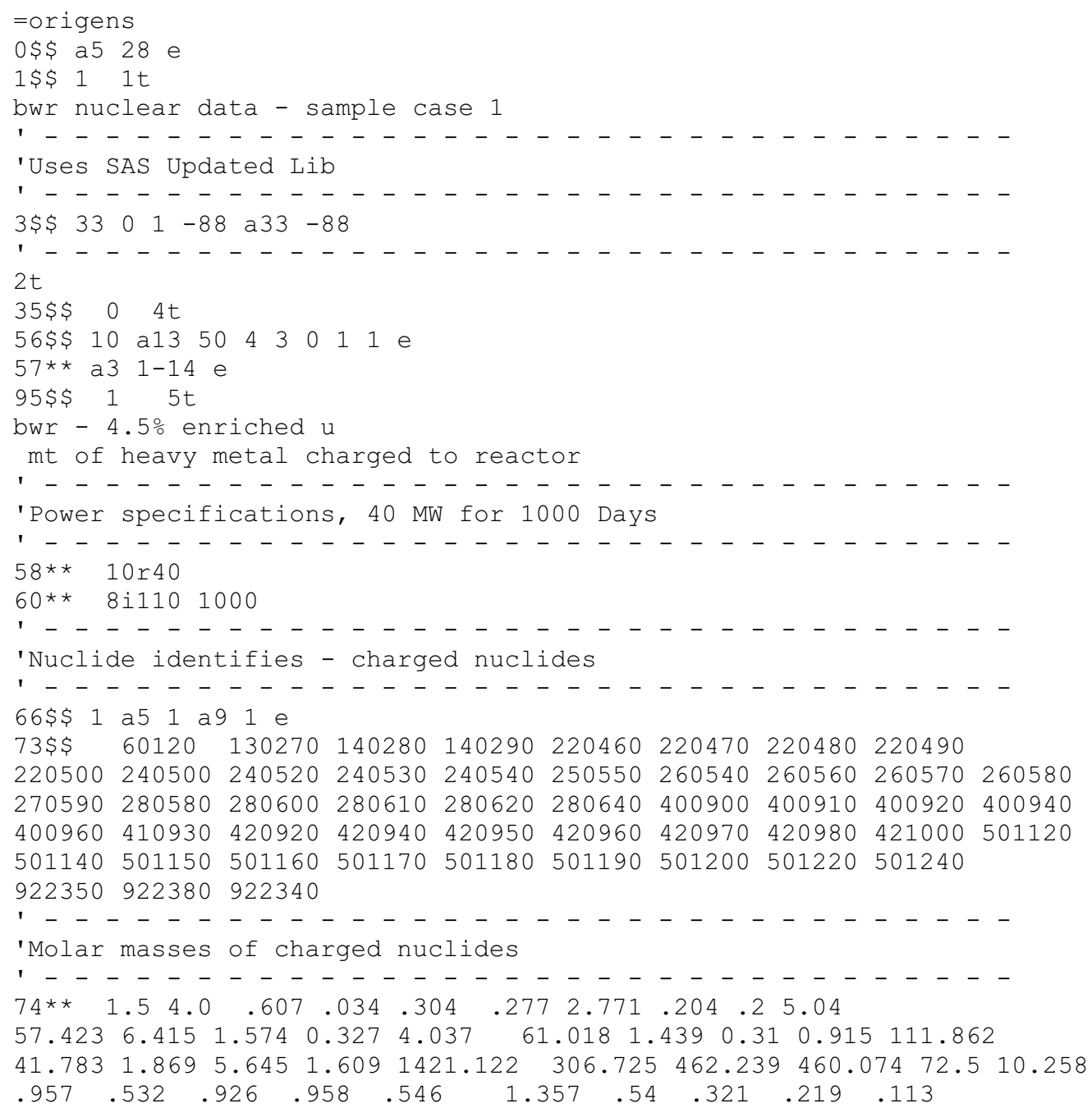

Figure A.III.2: ORIGEN Model, BWR, 4.5 w/o and 40 GWD/MTU 


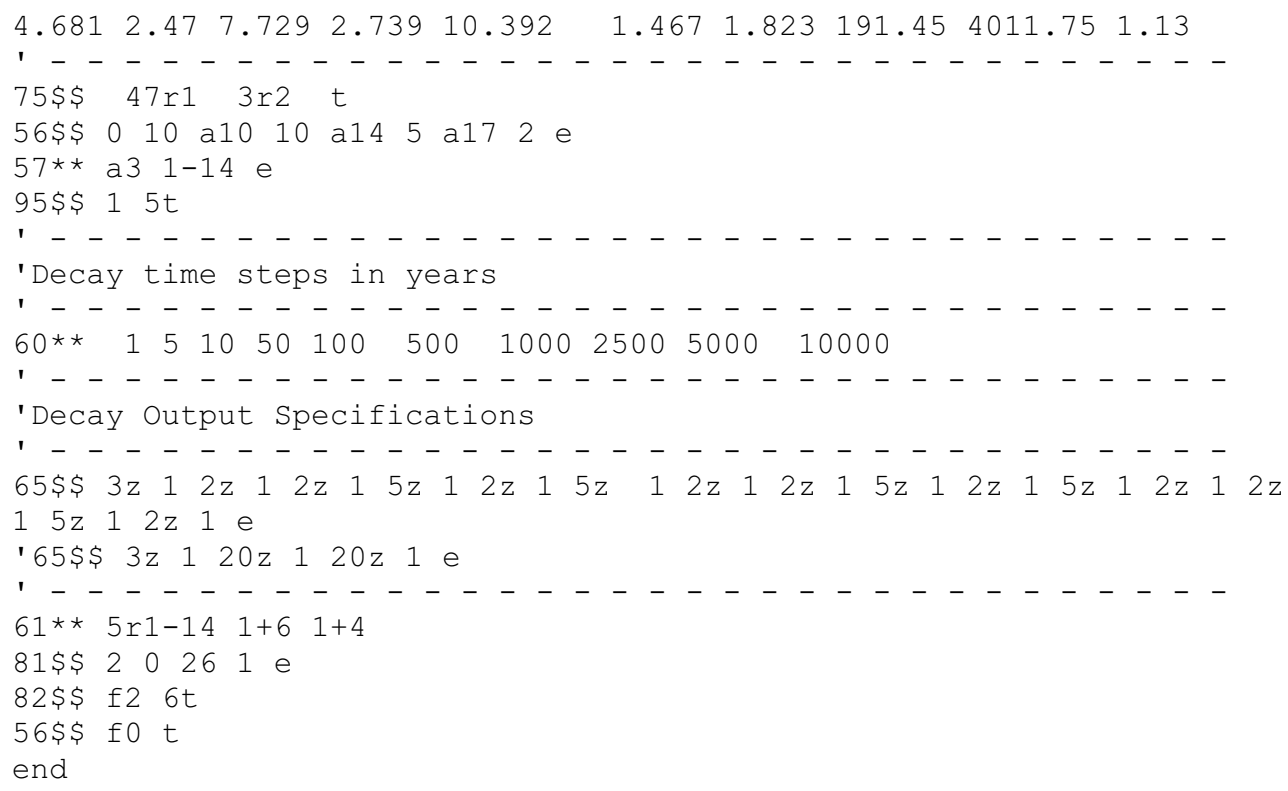

Figure A.III.2: ORIGEN Model, BWR, 4.5 w/o and 40 GWD/MTU, cont.

\section{The PWR Separation Model Using a UOX Fuel}

Since reprocessing hinges on chemical separation, we are also interested in uncertainty in the separation of SNF. Since chemistry only applied to elements we look at elemental uranium, neptunium, plutonium, americium, and curium - the key actinides produced by irradiating UOX fuel and those of interest to reprocessing and repository performance. This study examined heat loads produced by the separated elements in "lumps". That is, at a given time after irradiation the key isotopics for a certain element at that time are placed into ORIGEN and decayed for the typical time steps already mentioned in previous appendices and the total heat produced from that "lump", regardless of daughters, is examined. This is equivalent to chemically separating that element, with $100 \%$ efficiency assumed, at the given time and then sitting that element away to decay. The procedure is repeated with +1 standard deviation of the isotopics as defined by the uncertainty in the PWR UOX fuel model which yields a heat $+/$ - a heat uncertainty for each of the elements over their given decay times. A table of isotopics at 5, 10, and 25 years after irradiations is given in 
Table A.IV.1. Since the ORIGEN model used in this experiment is so general and uses a well known decay model, it is not presented.

\begin{tabular}{|c|c|c|c|c|c|c|}
\hline & \multicolumn{2}{|c|}{ Mass at 5 Years } & \multicolumn{2}{|c|}{ Mass at 10 Years } & \multicolumn{2}{|c|}{ Mass at 25 Years } \\
\hline Nuclide & Grams & +/- Grams & Grams & +/- Grams & Grams & +/- Grams \\
\hline u234 & $1.60 \mathrm{E}+02$ & $2.53 \mathrm{E}+00$ & $1.67 \mathrm{E}+02$ & $2.64 \mathrm{E}+00$ & $1.87 \mathrm{E}+02$ & $2.96 \mathrm{E}+00$ \\
\hline u235 & $1.23 E+04$ & $1.62 E+02$ & $1.23 E+04$ & $1.62 E+02$ & 1.23E+04 & $1.62 E+02$ \\
\hline u236 & $5.53 \mathrm{E}+03$ & $3.94 \mathrm{E}+01$ & $5.53 \mathrm{E}+03$ & $3.94 \mathrm{E}+01$ & $5.53 \mathrm{E}+03$ & $3.94 \mathrm{E}+01$ \\
\hline u237 & 3.75E-05 & $9.45 \mathrm{E}-07$ & 2.95E-05 & 7.42E-07 & 1.43E-05 & $3.60 \mathrm{E}-07$ \\
\hline u238 & $9.30 E+05$ & $7.21 E+02$ & $9.30 E+05$ & $7.21 E+02$ & $9.30 E+05$ & $7.21 E+02$ \\
\hline np237 & $5.69 \mathrm{E}+02$ & $3.44 \mathrm{E}+00$ & $5.73 E+02$ & $3.47 E+00$ & $5.95 \mathrm{E}+02$ & $3.60 \mathrm{E}+00$ \\
\hline np239 & 1.00E-04 & 1.37E-05 & 1.00E-04 & 1.37E-05 & 9.99E-05 & 1.36E-05 \\
\hline pu238 & $1.87 E+02$ & $1.96 \mathrm{E}+00$ & $1.80 E+02$ & $1.88 E+00$ & $1.60 \mathrm{E}+02$ & 1.67E+00 \\
\hline pu239 & $5.56 E+03$ & $4.48 E+01$ & $5.55 E+03$ & $4.48 E+01$ & $5.55 E+03$ & $4.48 E+01$ \\
\hline pu240 & $1.89 E+03$ & $4.85 E+01$ & $1.90 E+03$ & $4.86 E+01$ & $1.90 \mathrm{E}+03$ & $4.88 E+01$ \\
\hline pu241 & $1.24 \mathrm{E}+03$ & $3.12 E+01$ & $9.72 E+02$ & $2.45 E+01$ & $4.71 \mathrm{E}+02$ & $1.19 E+01$ \\
\hline pu242 & $5.58 E+02$ & $1.45 E+01$ & $5.58 E+02$ & $1.45 E+01$ & $5.58 \mathrm{E}+02$ & $1.45 E+01$ \\
\hline am241 & $3.79 E+02$ & $9.49 E+00$ & $6.41 \mathrm{E}+02$ & $1.60 E+01$ & $1.12 \mathrm{E}+03$ & $2.80 E+01$ \\
\hline am $242 m$ & 8.97E-01 & 1.95E-02 & 8.76E-01 & 1.90E-02 & 8.13E-01 & 1.77E-02 \\
\hline am243 & $1.16 E+02$ & $1.59 E+01$ & $1.16 E+02$ & $1.59 E+01$ & 1.16E+02 & $1.58 E+01$ \\
\hline cm242 & 8.07E-03 & 1.75E-04 & 2.28E-03 & 4.96E-05 & $2.12 \mathrm{E}-03$ & 4.60E-05 \\
\hline cm244 & $2.66 \mathrm{E}+01$ & $3.02 E+00$ & $2.20 \mathrm{E}+01$ & $2.49 E+00$ & $1.24 \mathrm{E}+01$ & $1.40 \mathrm{E}+00$ \\
\hline cm245 & $1.21 \mathrm{E}+00$ & $1.24 \mathrm{E}-01$ & $1.21 \mathrm{E}+00$ & $1.24 \mathrm{E}-01$ & $1.21 \mathrm{E}+00$ & 1.24E-01 \\
\hline
\end{tabular}

Table A.IV.1: Isotopics of discharged UOX fuel at 5, 10, and 25 years after irradiation.

\section{The PWR Model Using a MOX Fuel}

For this study it was assumed that the MOX fuel would be burned in a conventional PWR and thus the SAS2H model (Figure A.V.1) for this fuel has the same geometry and operating parameters as that of the PWR UOX model described above with the exception that fuel is now burned to $50 \mathrm{GWD} / \mathrm{MTHM}$ with a specific power of $23.070 \mathrm{MW}$ /assembly for 1000 days. There is still $461.4 \mathrm{~kg}$ of heavy metal in the fuel but it is now divided between $\mathrm{UO}_{2}$ and $\mathrm{PuO}_{2}$. The composition of the MOX fuel is taken from an AFCI report from Los Alamos National Lab for Fiscal Year 2003, in which the fuel composition used in this study is designated "ALWR-2." The $424.04 \mathrm{~kg}$ of uranium is in the proportions $1.40 \mathrm{w} / \mathrm{o} \mathrm{U}-235$, $98.572 \mathrm{w} / \mathrm{o} \mathrm{U}-238$ and $0.028 \mathrm{w} / \mathrm{o} \mathrm{U}-234$ and is volume fraction weighted at $\mathrm{VF}=0.82876$. 
The $37.36 \mathrm{~kg}$ of plutonium is in the proportions $1.655 \mathrm{w} / \mathrm{o} \mathrm{Pu}-238,61.751 \mathrm{w} / \mathrm{o} \mathrm{Pu}-239$, $24.701 \mathrm{w} / \mathrm{o} \mathrm{Pu}-240,3.248 \mathrm{w} / \mathrm{o} \mathrm{Pu}-241,8.645 \mathrm{w} / \mathrm{o} \mathrm{Pu}-242$ and is volume fraction weighted at $\mathrm{VF}=0.06978$. Since the sited report only specified a mass of $\mathrm{Pu}$ in the fuel, the isotopics vector was selected to approximate the discharged plutonium isotopic proportions of UOX fuel.

The corresponding ORIGEN model (Figure A.V.2), from which sampling is conducted, is one metric ton of the MOX fuel with approximately $271 \mathrm{~kg}$ of zirc 2 cladding. The ORIGEN model is set to deplete the fuel using the binary cross section library generated from the fuel specific SAS2H model. Note: cross section perturbations are introduced directly into this binary working library during the sampling procedure. Fuel specific power density is $50 \mathrm{MW} / \mathrm{MTHM}$ for a cycle length of 1000 days giving the same 50GWD/ MTHM burnup as the SAS2H Model. The ORIGEN model is set to "burn" the fuel for 10 equal time steps at $100 \%$ power and then print out decay isotopics at charge, discharge, 1, 5, 10, 50, 100, $500,1000,2500,5000$, and 10000 year times. ORIGEN also has the availability to print the decay heat, activity, and radiotoxicity for these times.

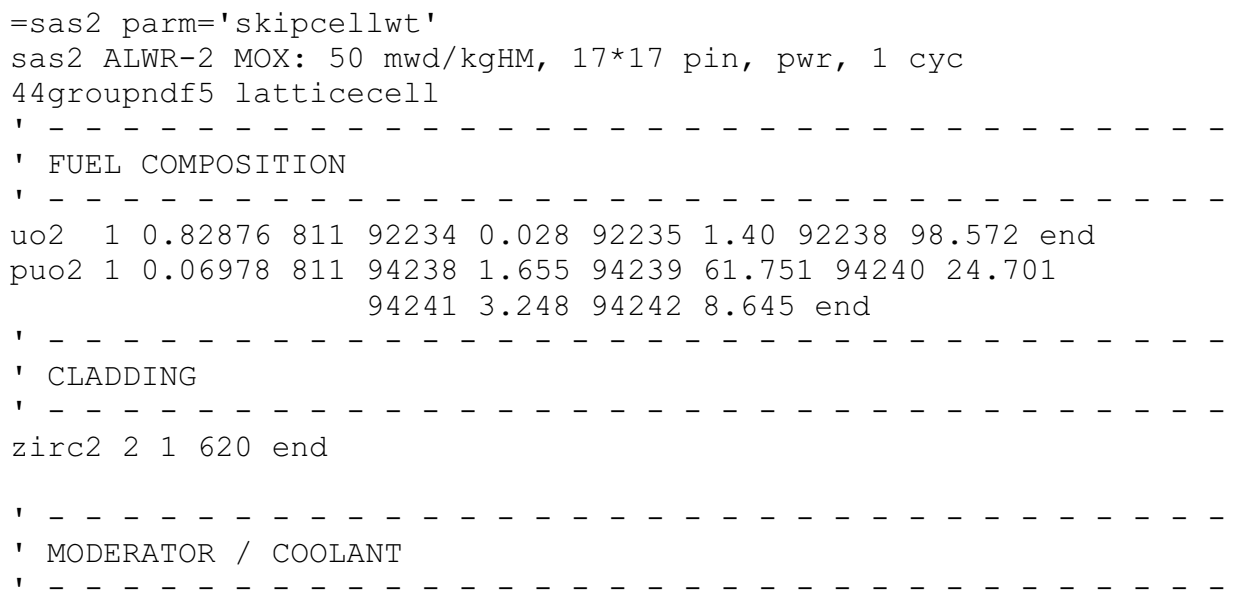

Figure A.V.1: SAS2H Model, MOX fuel in PWR, 50 GWD/MTHM 


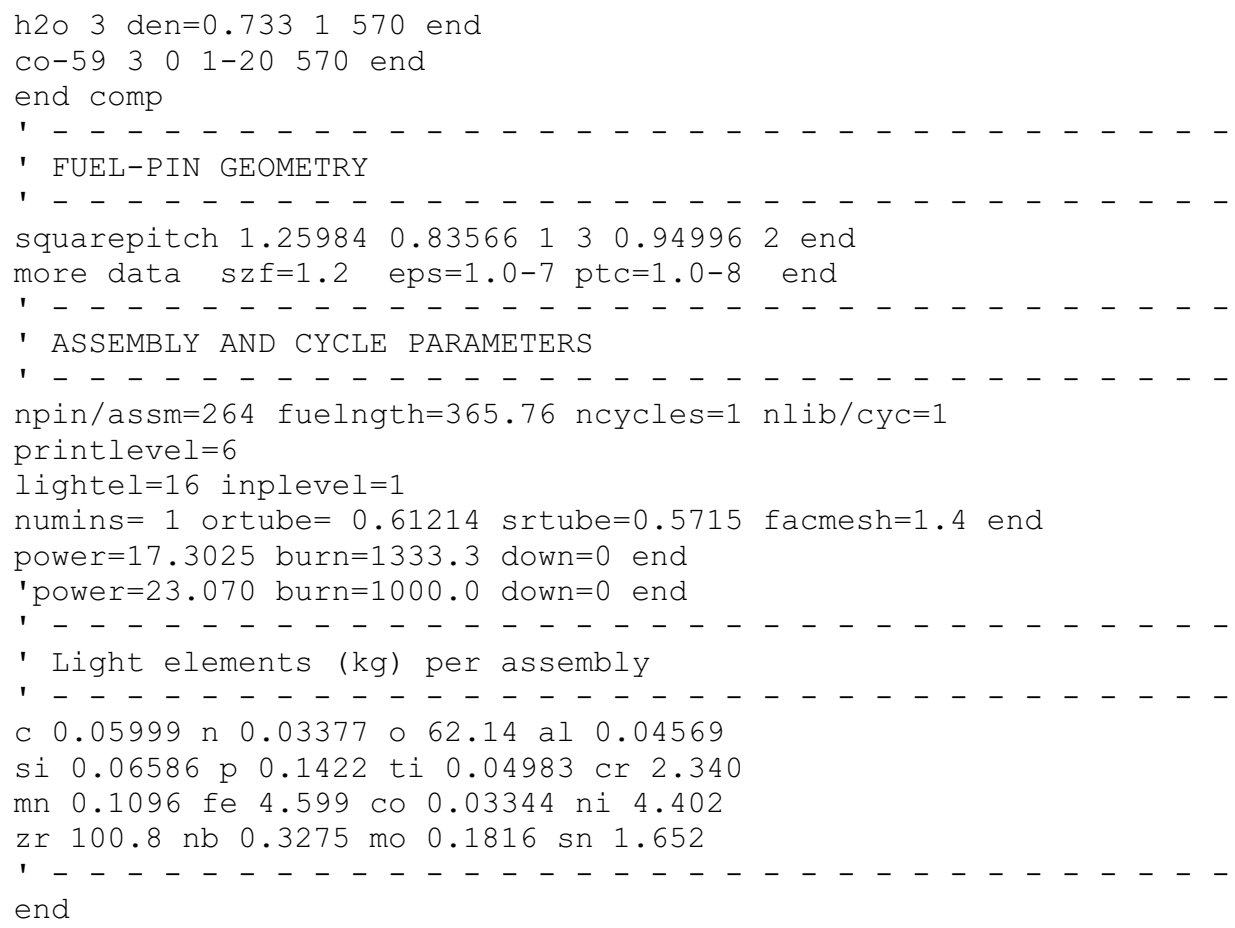

Figure A.V.1: SAS2H Model, MOX fuel in PWR, 50 GWD/MTHM, cont.

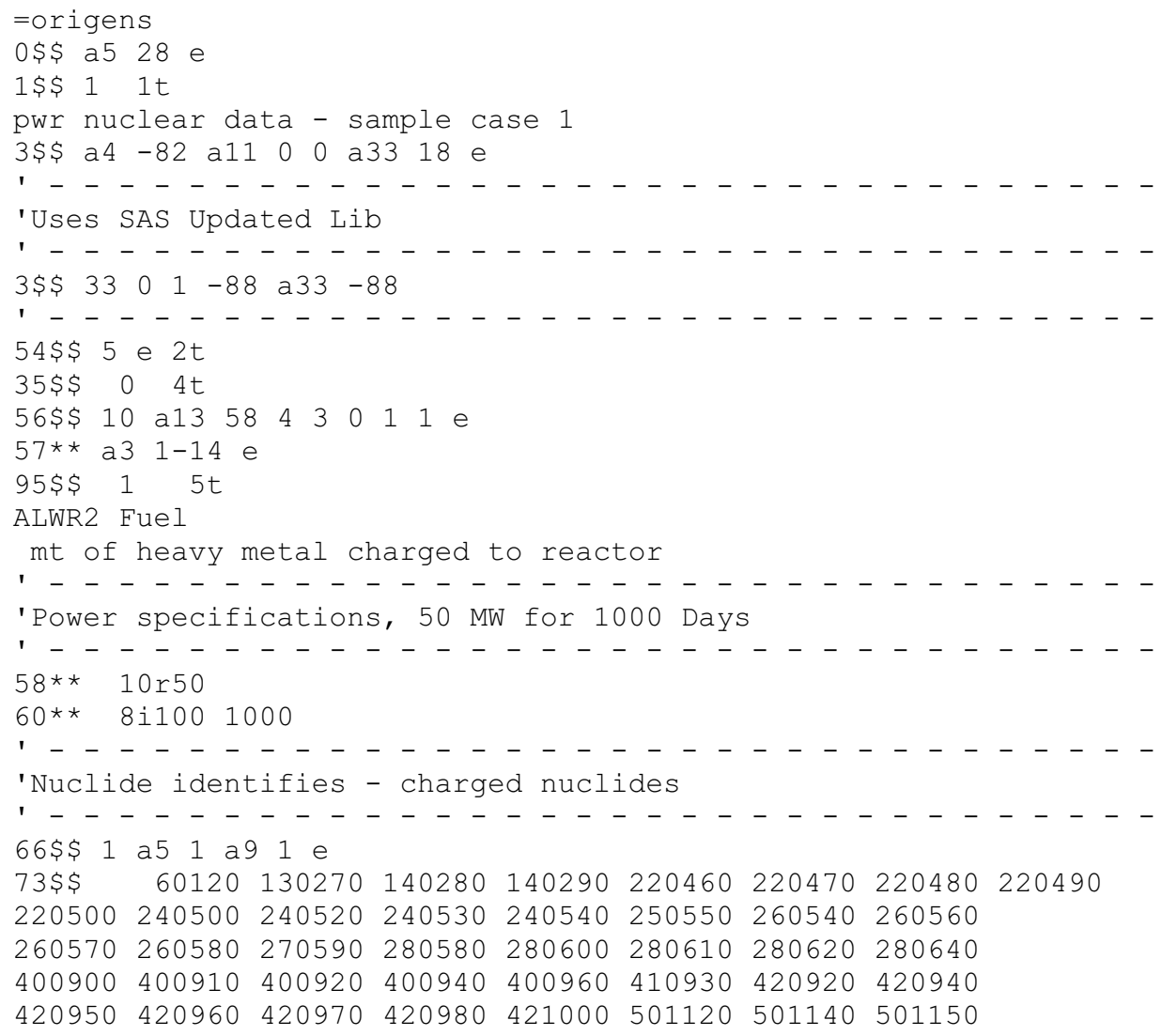

Figure A.V.2: ORIGEN Model, MOX fuel in PWR, 50 GWD/MTHM 


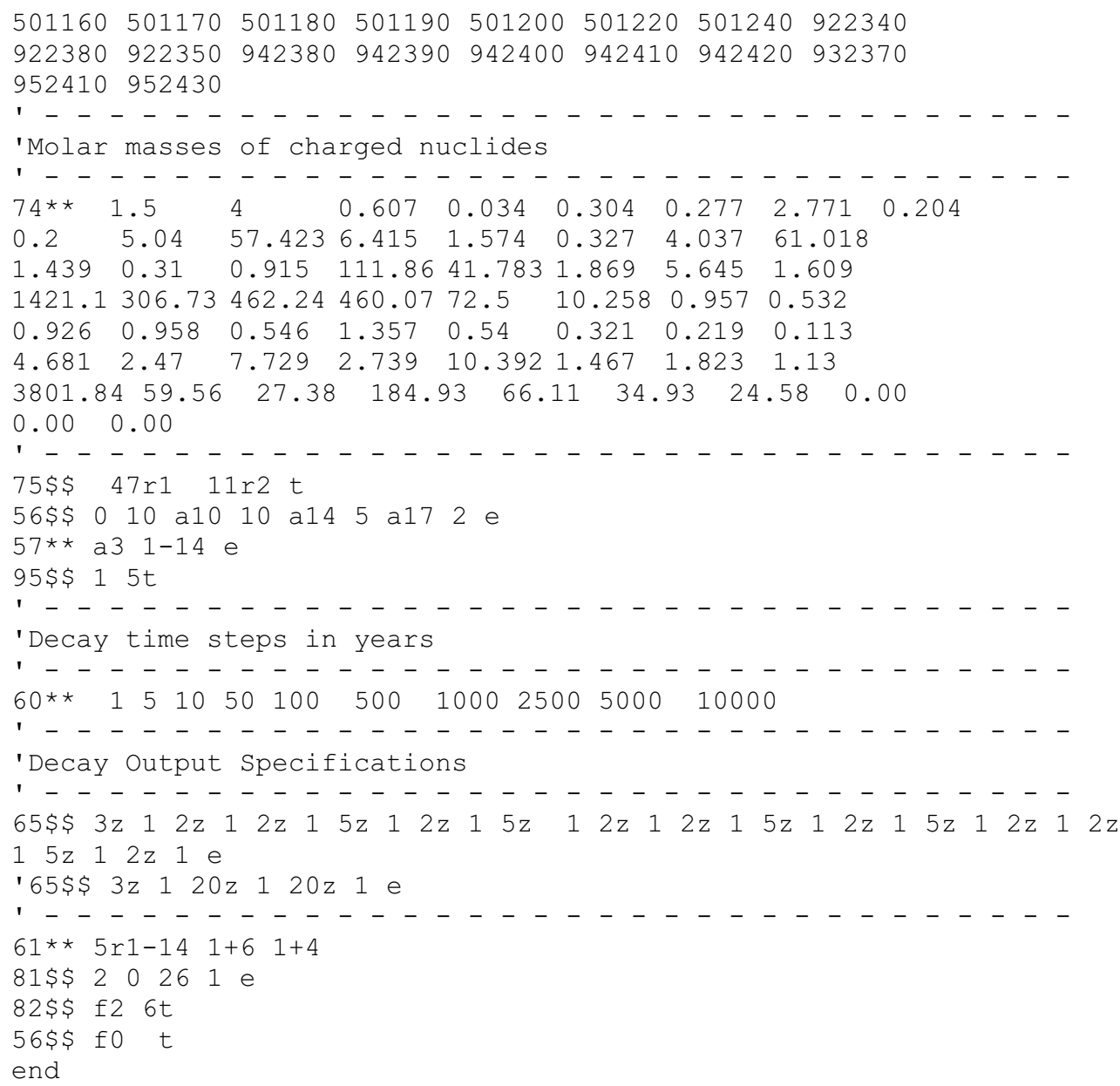

Figure A.V.2: ORIGEN Model, MOX fuel in PWR, 50 GWD/MTHM, cont.

\section{VI.The PWR Model Using a MOX Fuel with Impurities}

This model has the same geometry and operating conditions as the model described in the preceding section (Figure A.VI.1). The difference is now a portion of the U-238 mass has been removed and replaced with americium, Am, and neptunium, $\mathrm{Np}$, heavy metal impurities in the fuel in an effort to reflect a more realistic MOX fuel. The heavy metals have been added in the proportions $1 \mathrm{w} / \mathrm{o} \mathrm{Np}-237$ and $1.5 \mathrm{w} / \mathrm{o} \mathrm{Am}-241$ where the w/o is measured against the whole w/o of heavy metal in the fuel such that there is now $414.81 \mathrm{~kg}$ U, 37.36 kg Pu, 4.61 kg Np-237, and $6.92 \mathrm{~kg} \mathrm{Am-241.} \mathrm{Due} \mathrm{to} \mathrm{the} \mathrm{similarities} \mathrm{in} \mathrm{the} \mathrm{model}$ 
the only major change is the volume fraction of the $\mathrm{UO}_{2}$ and $\mathrm{PuO}_{2}$ and those of $\mathrm{Am}-241$ and Np-137 which are now $0.809,0.070,0.0096$, and 0.004 respectively.

The corresponding ORIGEN model (Figure A.VI.2), from which sampling is conducted, is one metric ton of the MOX fuel with approximately $271 \mathrm{~kg}$ of zirc 2 cladding. The ORIGEN model is set to deplete the fuel using the binary cross section library generated from the fuel specific SAS2H model. Note: cross section perturbations are introduced directly into this binary working library during the sampling procedure. Fuel specific power density is $50 \mathrm{MW} / \mathrm{MTHM}$ for a cycle length of 1000 days giving the same 50GWD/ MTHM burnup as the SAS2H Model. The ORIGEN model is set to "burn" the fuel for 10 equal time steps at $100 \%$ power and then print out decay isotopics at charge, discharge, 1, 5, 10, 50, 100, $500,1000,2500,5000$, and 10000 year times. ORIGEN also has the availability to print the decay heat, activity, and radiotoxicity for these times.

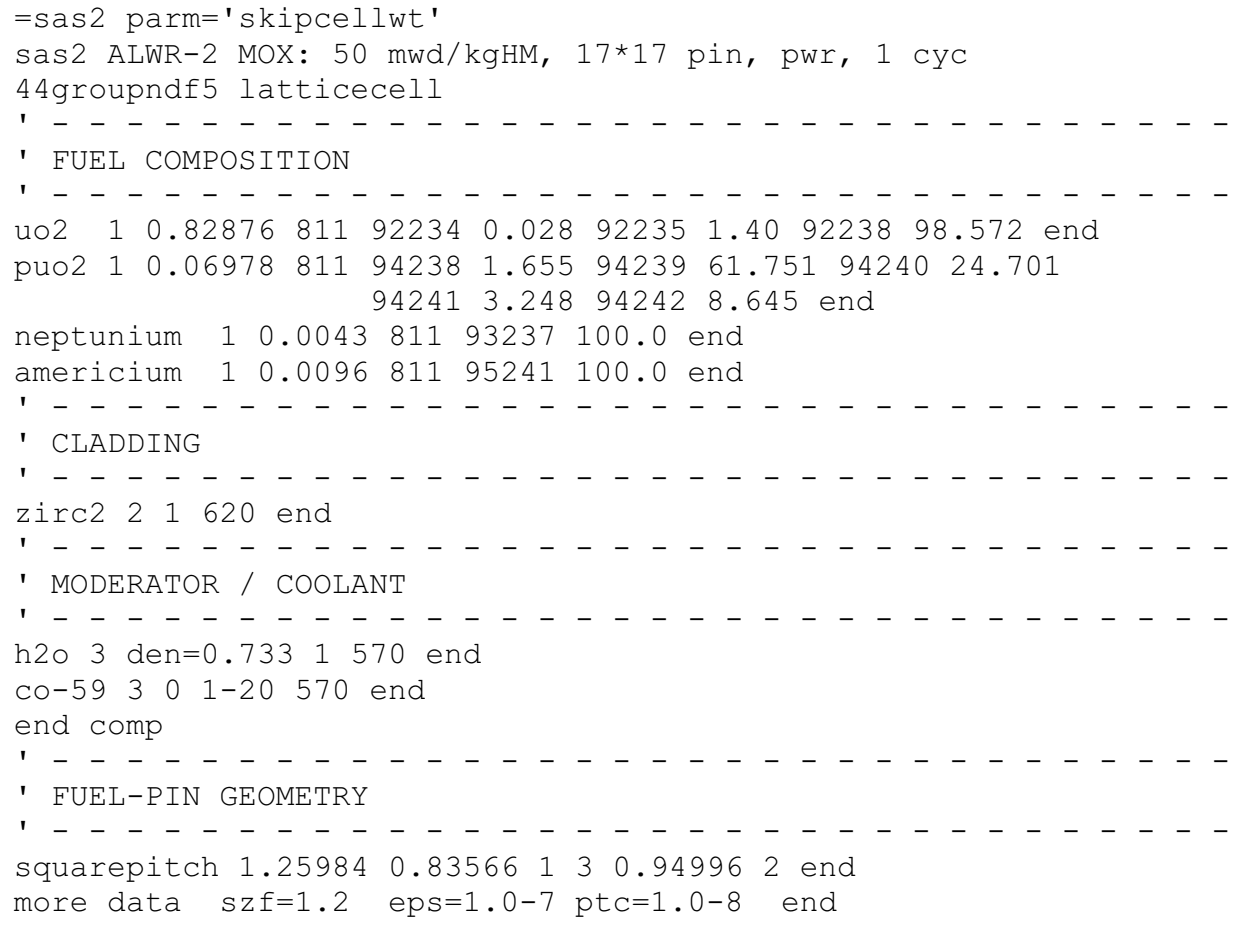

Figure A.VI.1: SAS2H Model, MOX with impurities fuel in PWR, 50 GWD/MTHM 


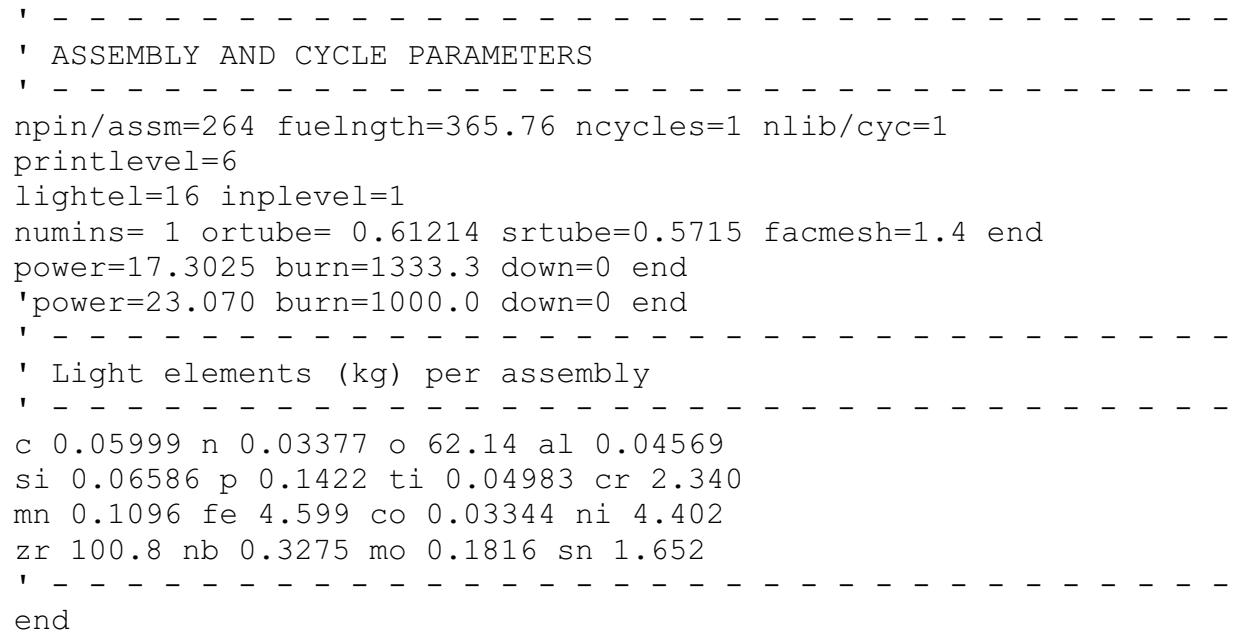

\section{Figure A.VI.1: SAS2H Model, MOX w/ impurities fuel in PWR, 50 GWD/MTHM, cont.}

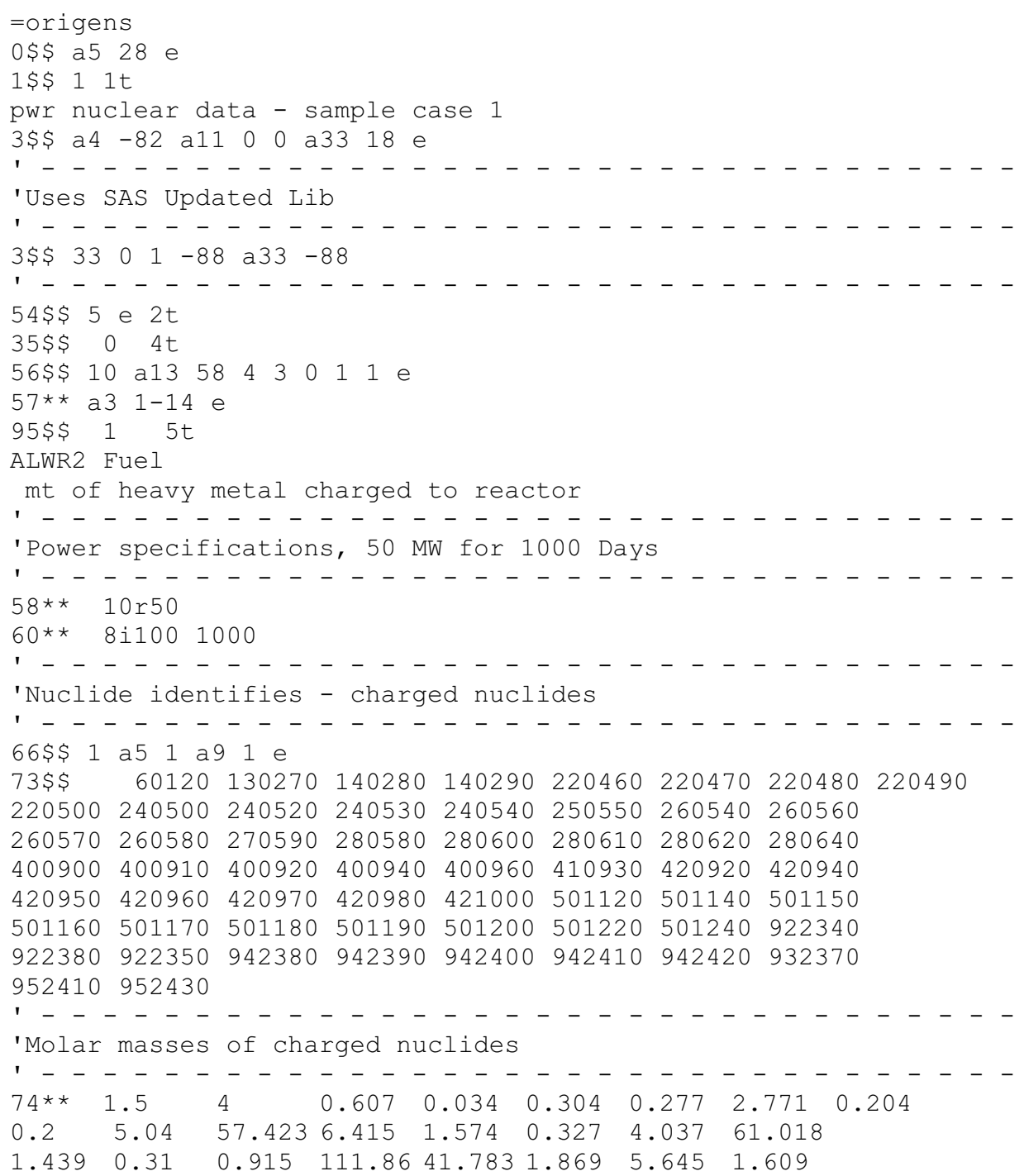

Figure A.VI.2: ORIGEN Model, MOX with impurities fuel in PWR, 50 GWD/MTHM 


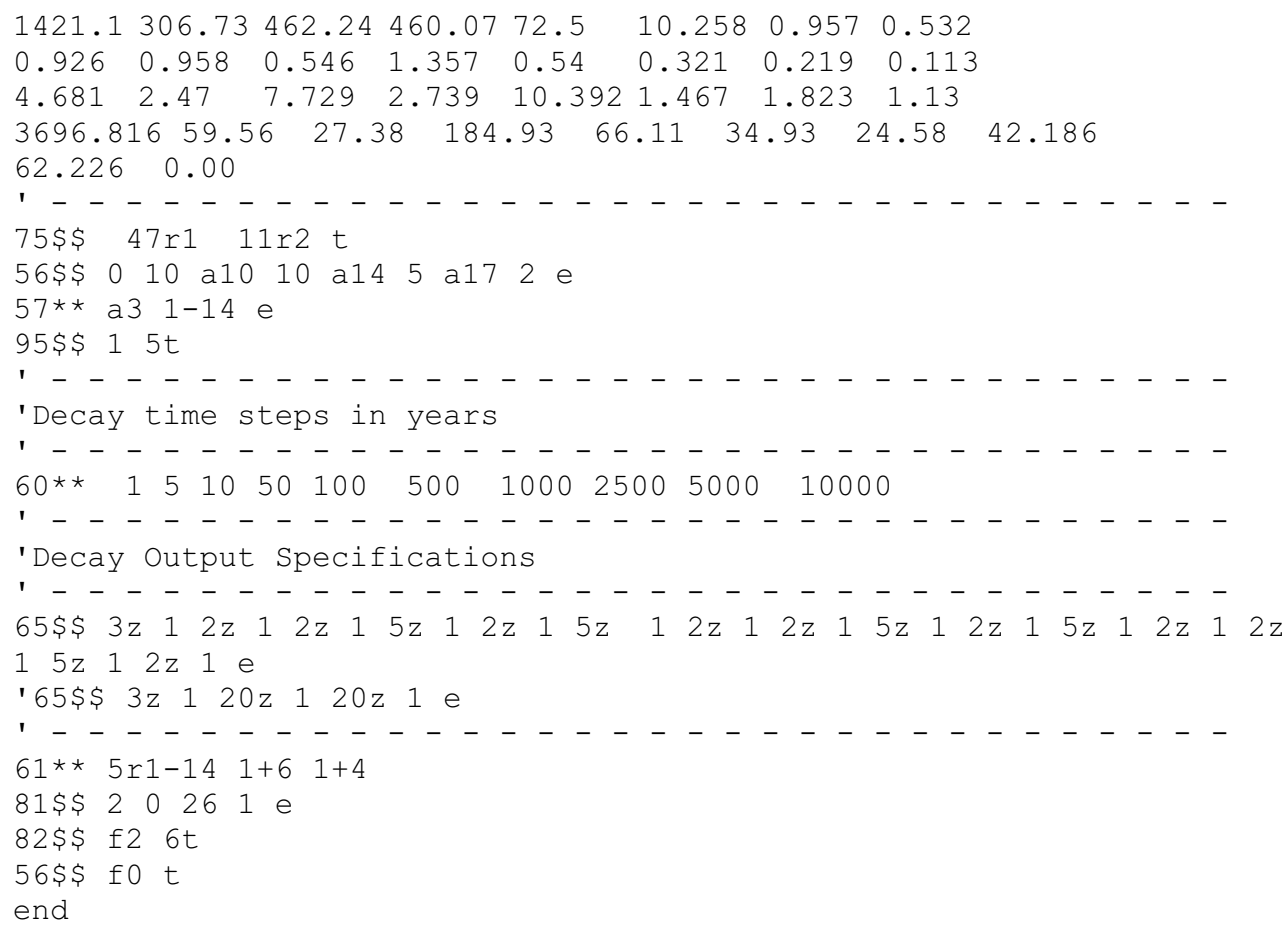

Figure A.VI.2: ORIGEN Model, MOX with impurities in PWR, 50 GWD/MTHM, cont.

\section{The PWR Model Using TRITON}

For purposes of conducting a comparison between using the SAS + ORIGEN scheme and using the driver program TRITON stand-alone, a TRITON model of the PWR fuel was created. The TRITON model represents a step up in the detail of modeling to a level closer to that of normal fuel analysis, in which a 2-D transport model is solved for each time step and new fluxes used to collapse a 44-group cross section library. A Wigner cell of the PWR fuel including the buffer region of extra water added by the water holes in the assembly is modeled. Two PWR models were constructed differing in the burnup steps while keeping all parameters are the same as the above model. The first model used a single burnup step the same as the cruder SAS2H model already discussed; the second, utilizing the robustness of TRITON, employed 26 burn-up steps which is closer to a realistic model of the fuel. Since 
only one line in the model is different only one copy of it is presented, given here in Figure

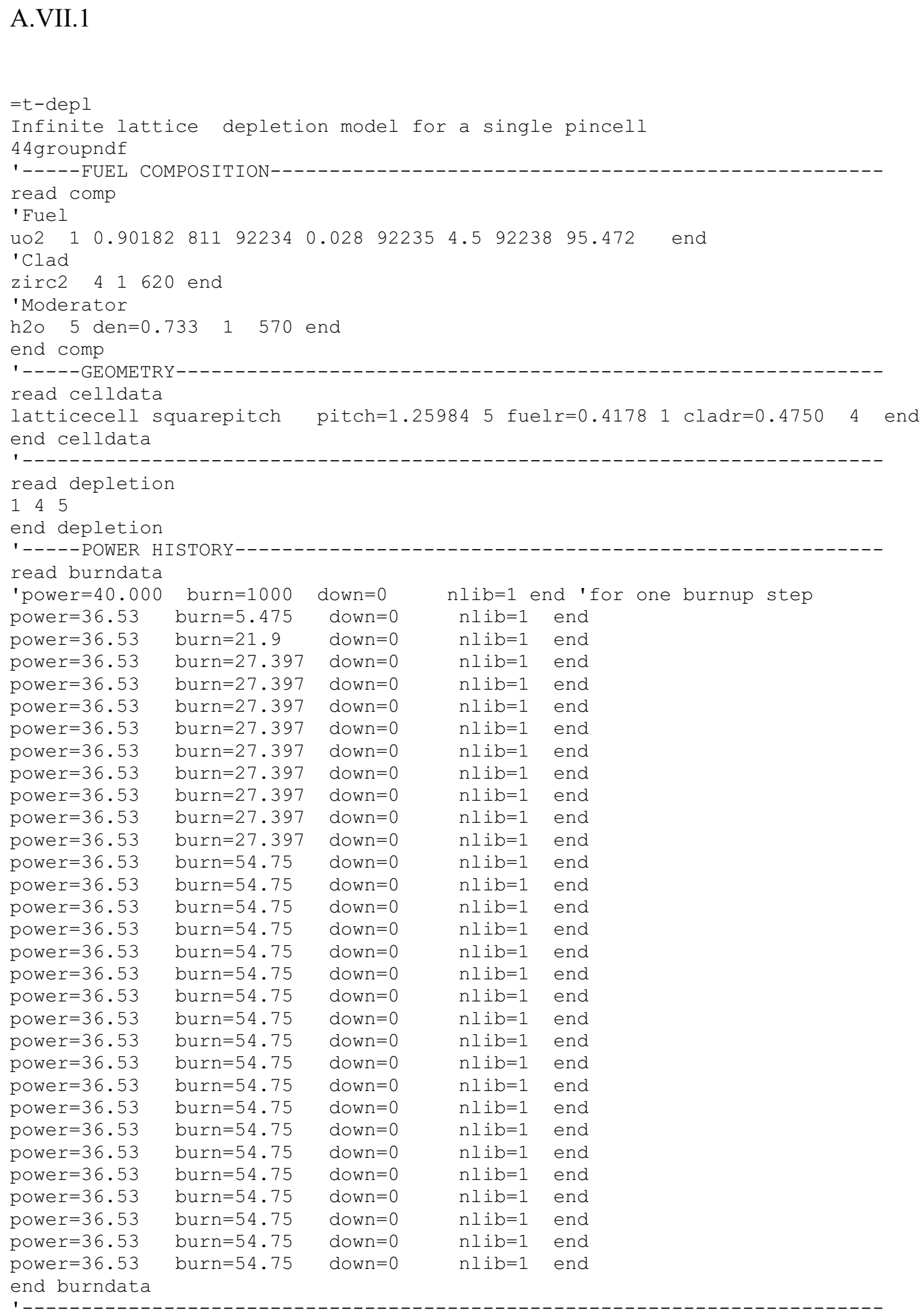

Figure A. VII.1: TRITON Model, PWR, 4.5 w/o and 48 GWD/MTU 


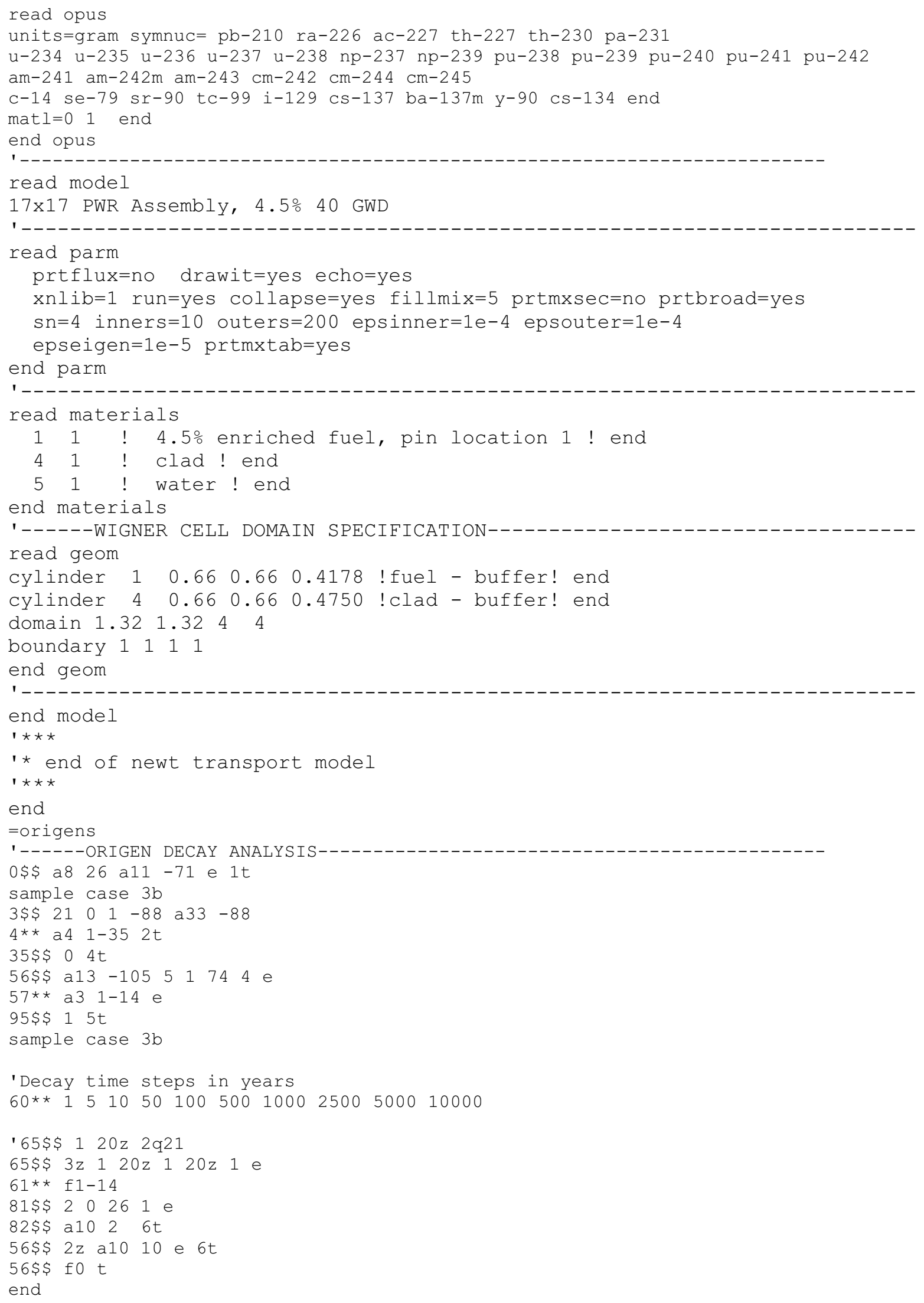

Figure A. VII.1: TRITON Model, PWR, 4.5 w/o and 40 GWD/MTU, cont. 


\section{TRITON FR Models Using Actinide Fuels}

The fast reactor, FR models used in this study was created in reference to various fuel assemblies for Argonne National Lab's Advanced Burner Test Reactor (ABTR) which would use a fuel whose isotopics are based on 10 year decayed UOX that was $3.3 \mathrm{w} / \mathrm{o}$ fresh fuel and burned for 33 GWD/MTU. The metal fuel consists of depleted uranium, the transuranics: neptunium, plutonium, americium, and curium metals, and 10-20 w/o zirconium mixed into the metal. Three fuel types are analyzed that are intended to have conversion ratios of 0.25 , 0.70 and 1.05 which are controlled by the TRU enrichment. Common among these assemblies are that the pins are in a triangular (hexagonal) pitch with an active fuel length of $80 \mathrm{~cm}$. The composition data including volume fractions are included in Table A.VIII.1. The cladding is a material developed by Argonne and has the composition shown in Table A. VIII.2; it is namely an iron alloy. Finally, as with several other fast reactor design concepts, the coolant for this model is elemental sodium. Operating temperatures are 909, 783 and 783 degrees Fahrenheit for the fuel, clad and moderator, respectively, with the moderator density at $7.97 \mathrm{~g} / \mathrm{cm}^{3}$. Specific powers, burnups, geometry and other important data are given in Table A. VIII.3. Note, TRITON automatically returns results in terms of 1 MTHM and the ORIGEN decay sequence is set for the standard charge, discharge, 1, 5, 10, 50, 100, 500, $1000,2500,5000$, and 10000 year time steps. The TRITON models for $\mathrm{CR}=1.05,0.70$, and 0.25 are presented in Figures A. VIII.1-3 respectively. For recycle sampling, consider these same models with input isotopics perturbed. 


\begin{tabular}{|c|c|c|c|}
\hline \multirow[b]{2}{*}{ Nuclide / Conversion Ratio: } & \multicolumn{3}{|c|}{ Weight Percent in TRU } \\
\hline & 0.25 & 0.7 & 1.05 \\
\hline $\mathrm{Np}-237$ & 18.635 & 7.334 & 9.907 \\
\hline Pu-238 & 0.855 & 1.253 & 0.000 \\
\hline Pu-239 & 32.764 & 48.058 & 72.150 \\
\hline Pu-240 & 14.983 & 21.973 & 4.469 \\
\hline $\mathrm{Pu}-241$ & 4.936 & 7.241 & 0.250 \\
\hline Pu-242 & 2.956 & 4.335 & 0.000 \\
\hline Am-241 & 20.579 & 8.100 & 10.941 \\
\hline$A m-242 m$ & 0.041 & 0.016 & 0.022 \\
\hline Am-243 & 3.565 & 1.403 & 1.895 \\
\hline $\mathrm{Cm}-244$ & 0.689 & 0.271 & 0.366 \\
\hline $\mathrm{Cm}-245$ & 0.041 & 0.016 & 0.022 \\
\hline Fissile Fraction, $\%$ & 37.7 & 55.30 & 72.40 \\
\hline TRU Enrichment, \% & 59.2 & 20.6 & 16.2 \\
\hline Zr w/o & 20 & 10 & 10 \\
\hline U-238, w/o & 20.8 & 69.4 & 73.8 \\
\hline
\end{tabular}

Table A.VIII.1: Fast Reactor Fuel Composition Data, by conversion ratio

\begin{tabular}{|l|c|}
\hline \multicolumn{2}{|c|}{ Cladding Composition } \\
\hline Material & mass $/ \mathbf{c m}^{\mathbf{3}}$ \\
\hline Iron & $7.10 \mathrm{E}-02$ \\
\hline Nickel & $4.38 \mathrm{E}-04$ \\
\hline Chromium & $1.06 \mathrm{E}-02$ \\
\hline Manganese-55 & $4.68 \mathrm{E}-04$ \\
\hline Molybdenum & $4.99 \mathrm{E}-04$ \\
\hline
\end{tabular}

Table A. VIII.2: Cladding Composition Data

\begin{tabular}{|l|r|r|r|}
\hline Conversion Ration & $\mathbf{0 . 2 5}$ & $\mathbf{0 . 7 0}$ & $\mathbf{1 . 0 5}$ \\
\hline Specific Power of active core, MW/MT & 114.8 & 47.7 & 41.2 \\
\hline Discharge Burnup, GWD.MT & 94.3 & 78.4 & 67.7 \\
\hline Height, cm & 80 & 80 & 80 \\
\hline Number of pins per assembly & 217 & 169 & 127 \\
\hline Assembly lattice pitch, cm & 14.834 & 14.834 & 14.834 \\
\hline Inter-assembly gap, mm & 4.45 & 4.0 & 4.0 \\
\hline Duct thickness, mm & 4.45 & 3.0 & 3.0 \\
\hline Pin pitch-to-diameter ratio & 1.29 & 1.11 & 1.10 \\
\hline Cladding thickness, mm & 0.75 & 0.41 & 0.41 \\
\hline
\end{tabular}

Table A. VIII.3: Operating Conditions and Geometry Data 


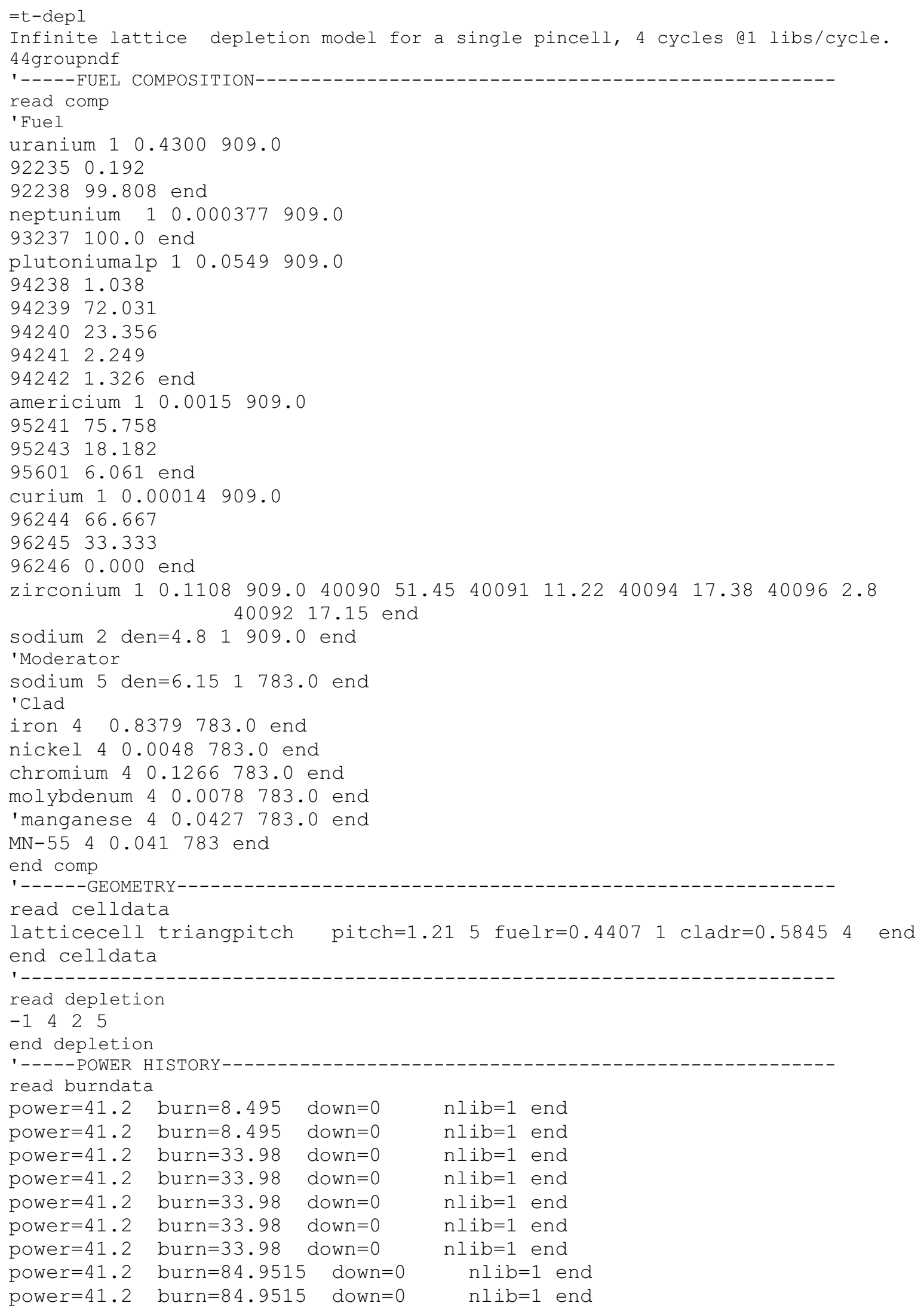

Figure A. VIII.1: TRITON Model, $C R=1.05$ 


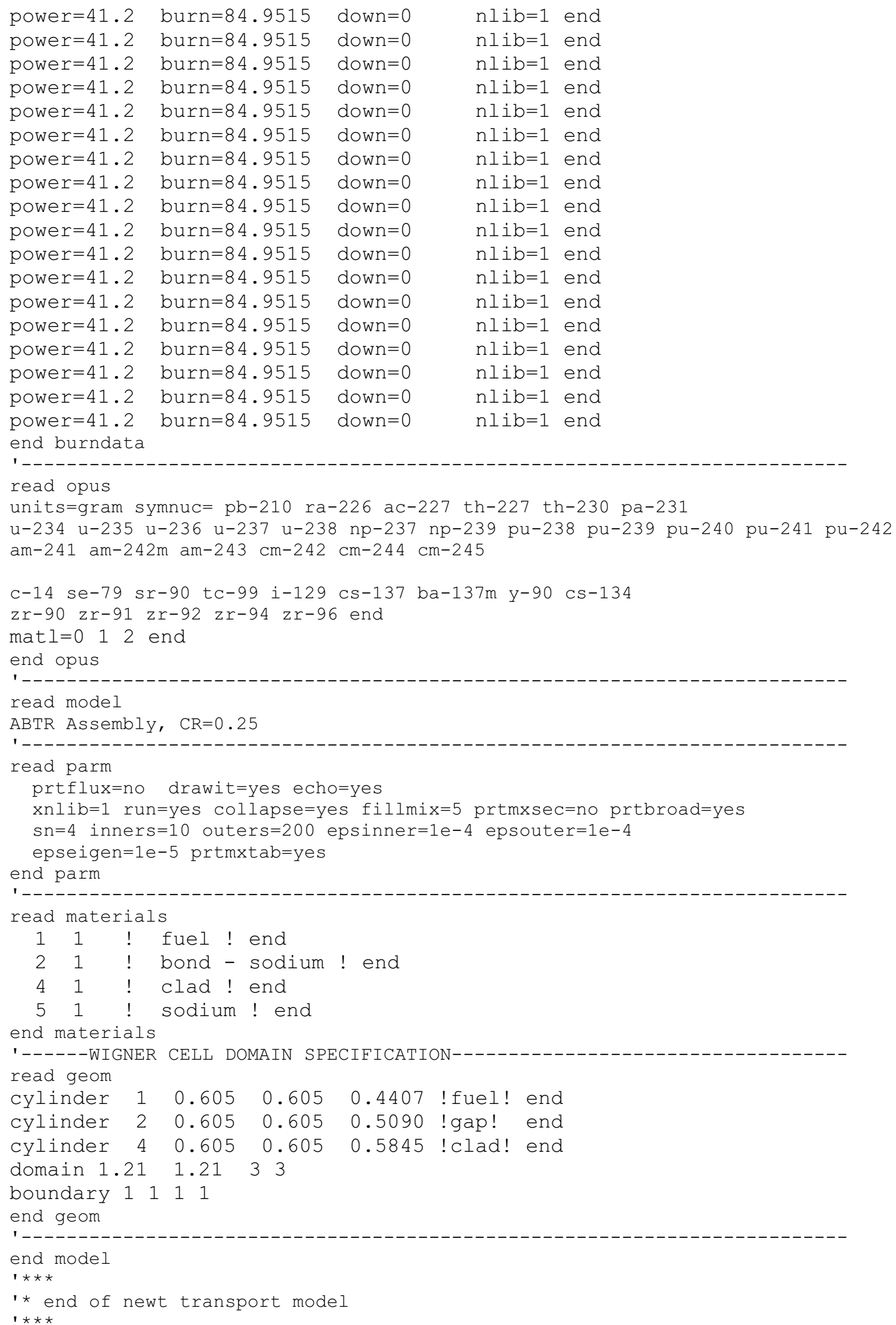

Figure A. VIII.1: TRITON Model, $C R=1.05$, cont. 


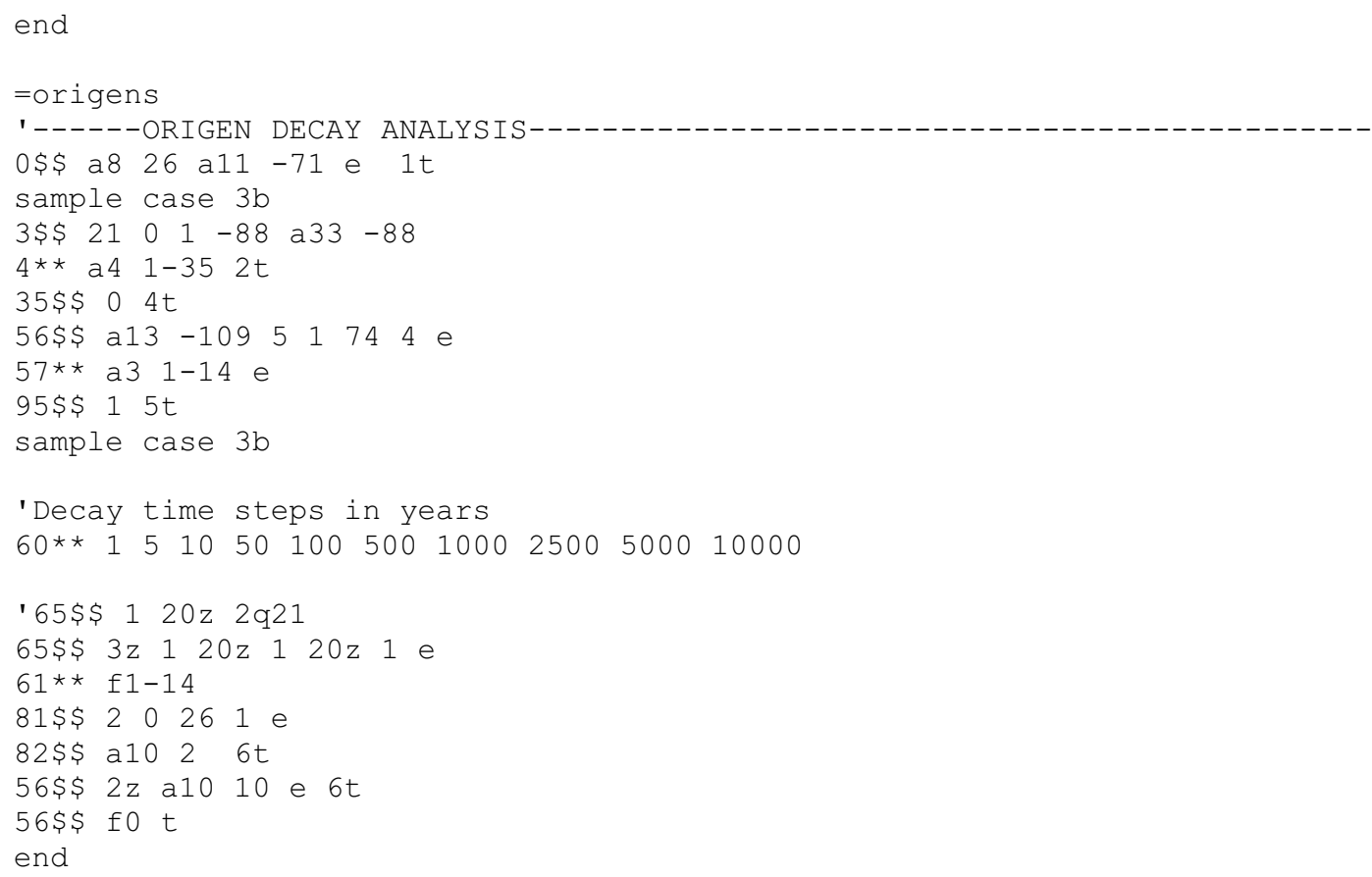

Figure A. VIII.1: TRITON Model, $C R=1.05$, cont.

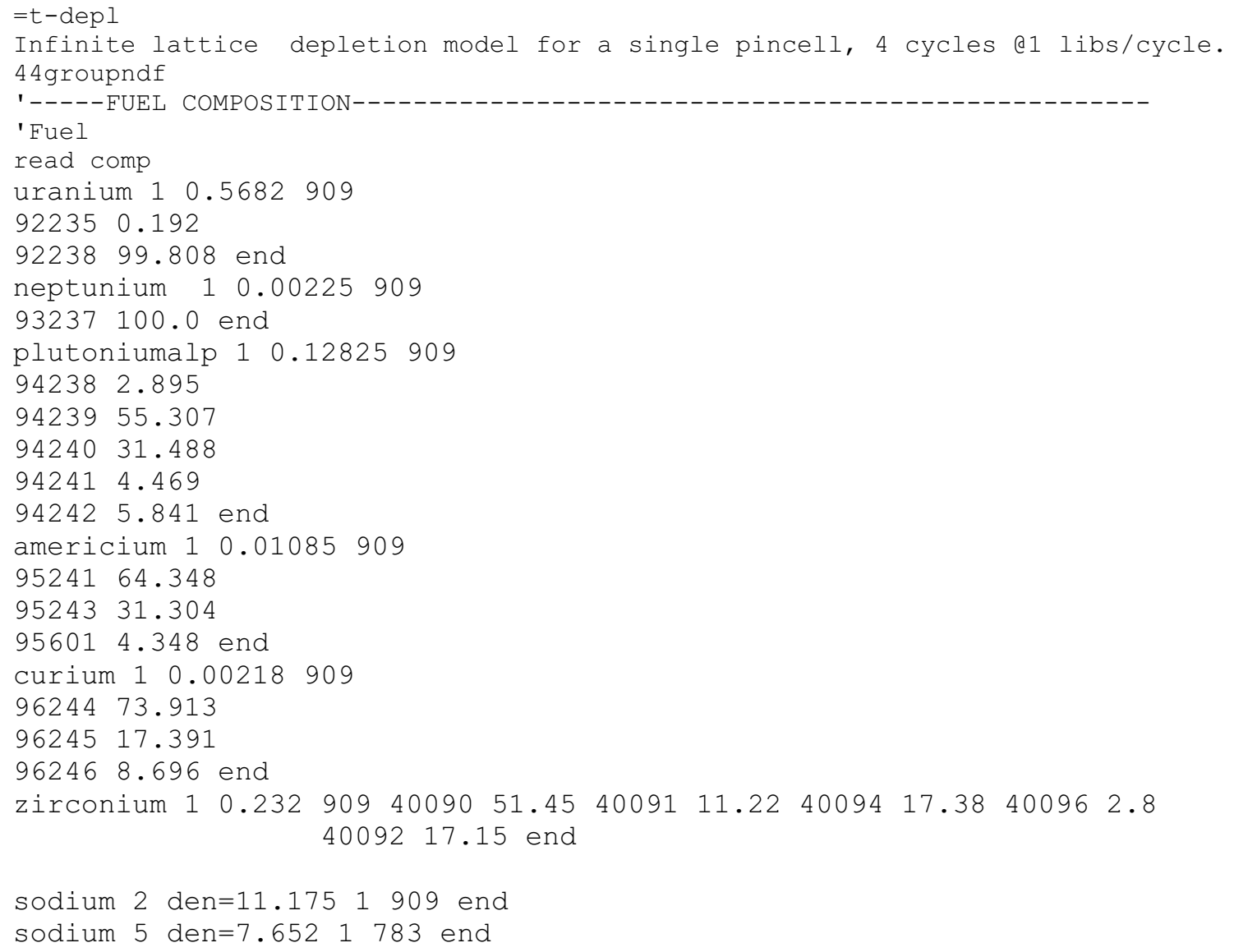

Figure A. VIII.2: TRITON Model, $C R=0.70$ 


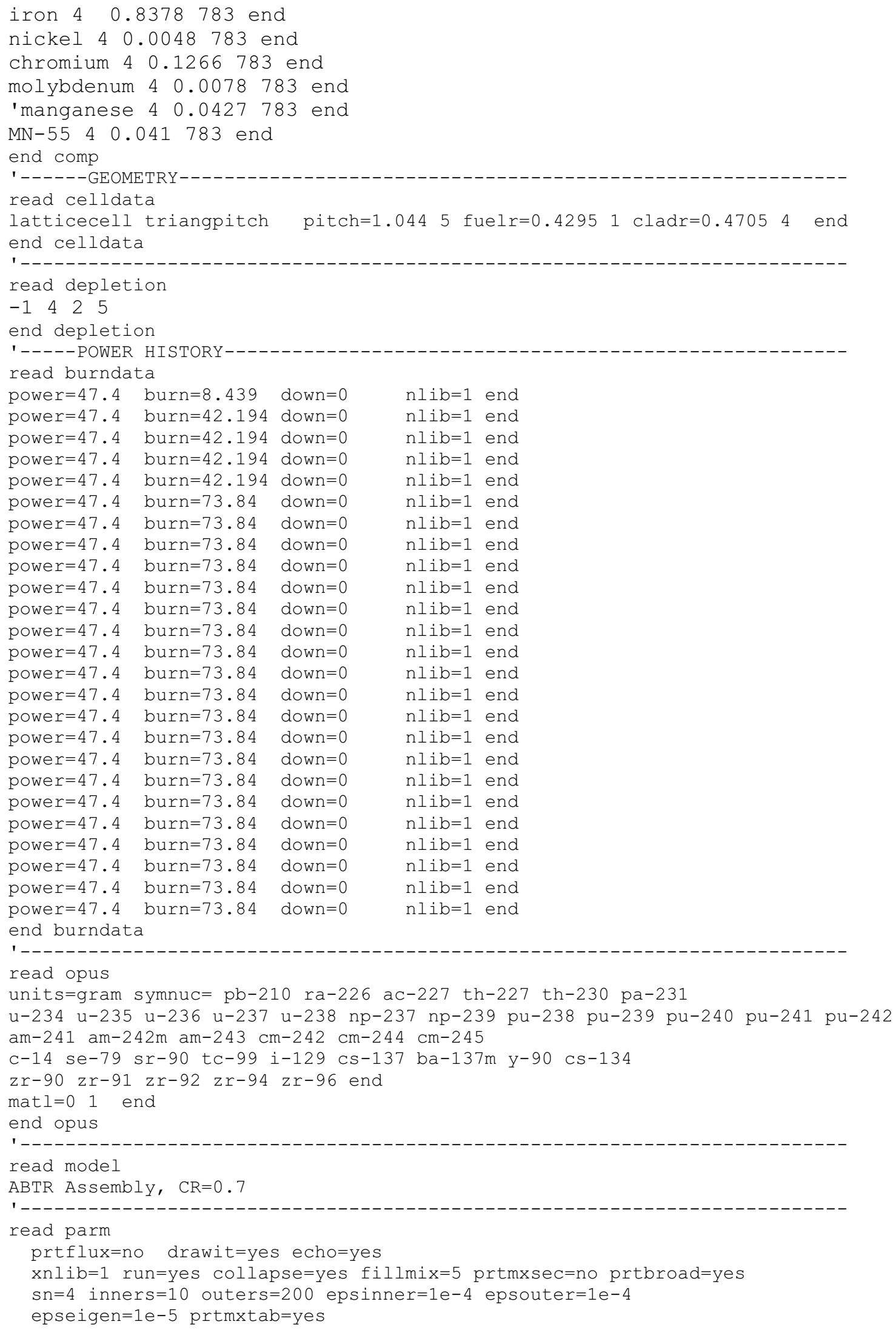

Figure A. VIII.2: TRITON Model, $\mathbf{C R}=\mathbf{0 . 7 0}$, cont. 


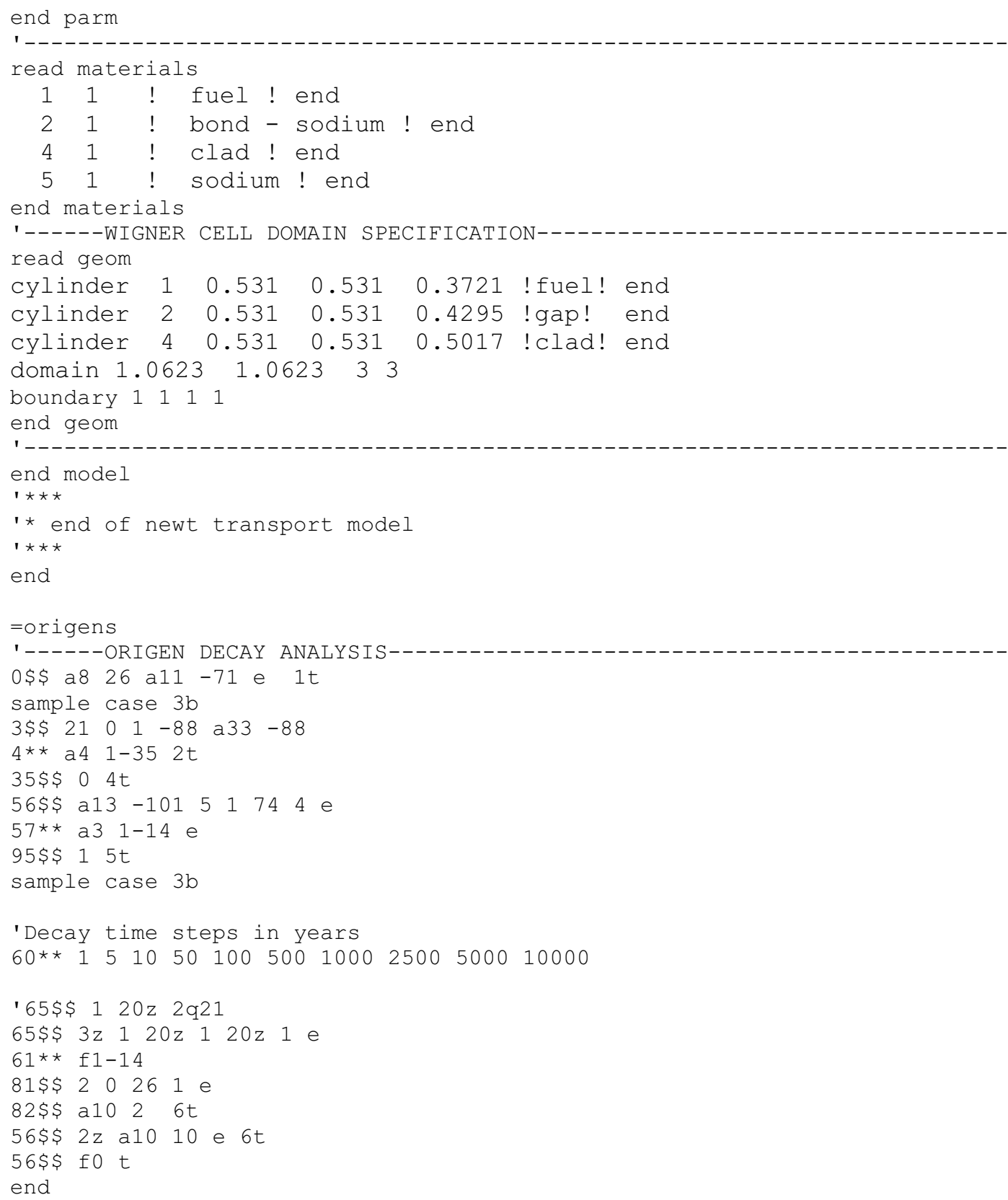

Figure A. VIII.2: TRITON Model, $\mathbf{C R}=\mathbf{0 . 7 0}$, cont.

$=\mathrm{t}-\mathrm{dep} I$

Infinite lattice depletion model for a single pincell, 4 cycles el libs/cycle. 44 groupndf

Figure A. VIII.3: TRITON Model, $\mathrm{CR}=\mathbf{0 . 2 5}$ 


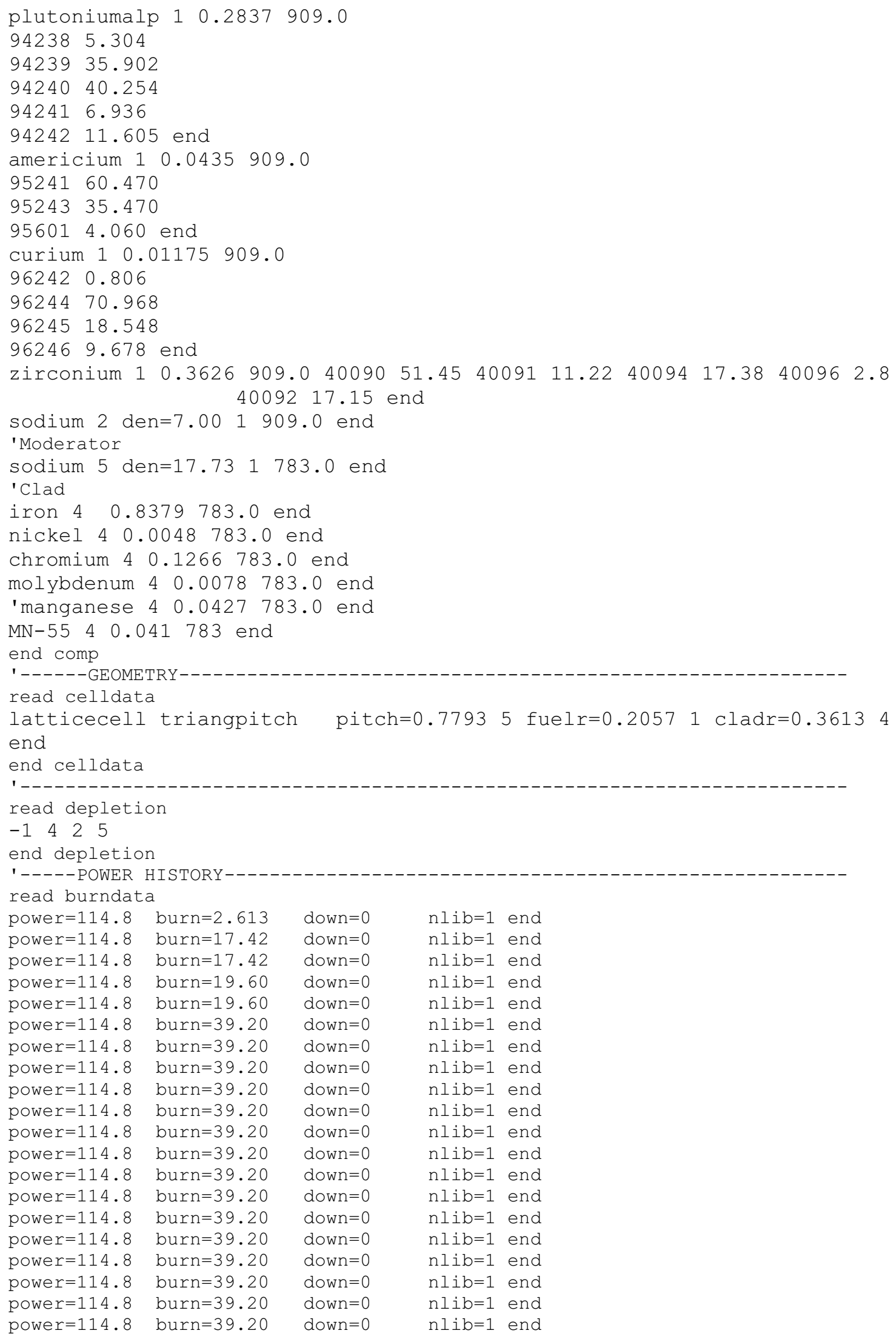

Figure A. VIII.3: TRITON Model, $C R=0.25$, cont. 


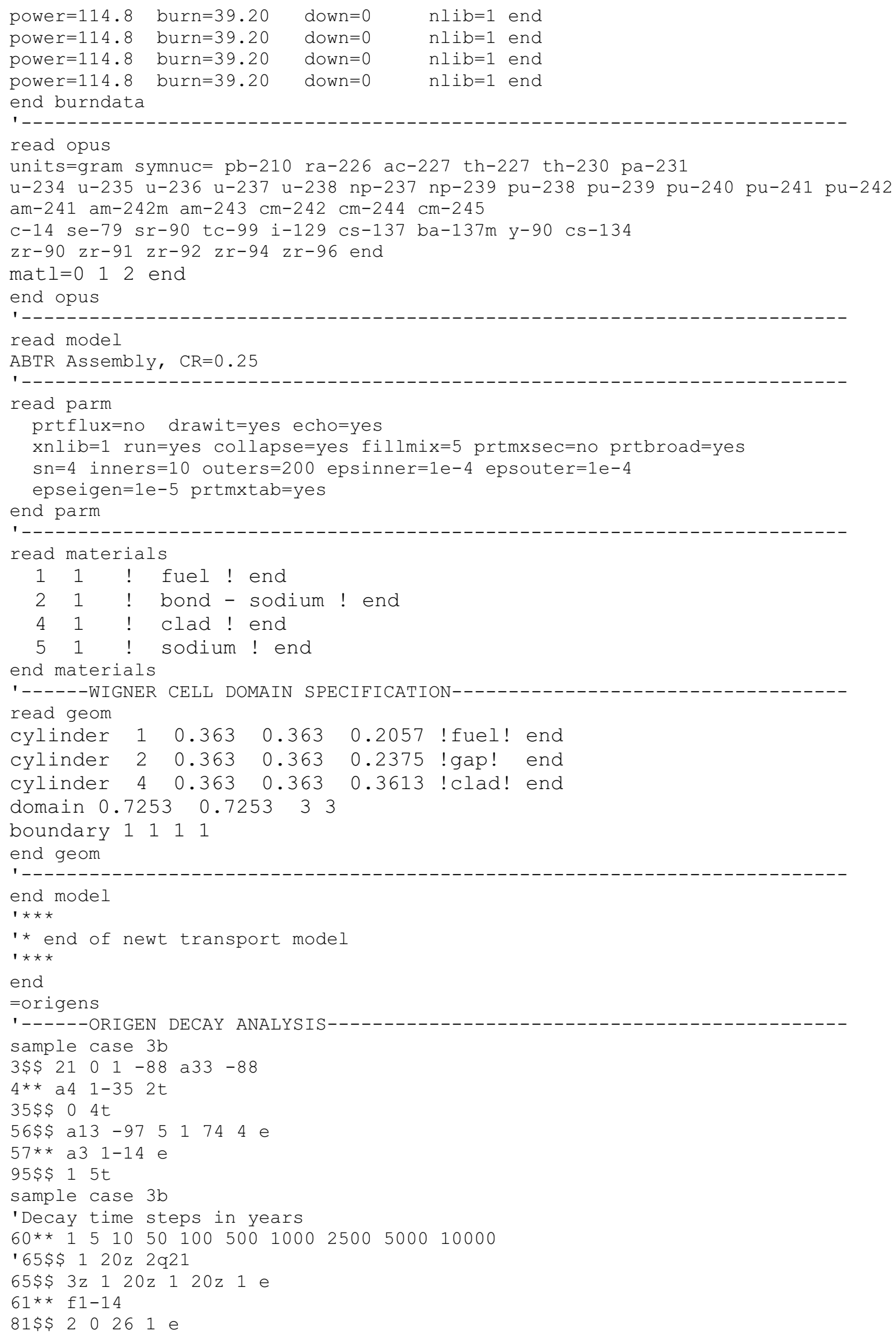

Figure A. VIII.3: TRITON Model, $C R=0.25$, cont. 
Figure A. VIII.3: TRITON Model, $\mathrm{CR}=0.25$, cont.

Since the TRITON model used for the recycling experiment is the same as the $\mathrm{CR}=$ 0.70 fast reactor model, it is not repeated here. The burnup is adjusted to 41.4 GWD/MTHM, which is the end of cycle core average burnup, and the input isotopics for each element are different at each recycle step.

\section{IX.REBUS Fast Reactor Model with Actinide Fuel and Recycle}

The REBUS model was set up to reproduce the fuel loading and recycle as specified by Argonne's ABTR Preconceptual Design [26] report for the medium conversion ratio core. The model is set to recycle all of the fast reactor fuel transuranics after 1.5 years of cooling, and make up the mass and reactivity by using spent LWR fuel and depleted uranium. As described in the Results section, this model more closely matched the values in the report in terms of loading and operating parameters. The complete input is given in Figure A.IX.1.

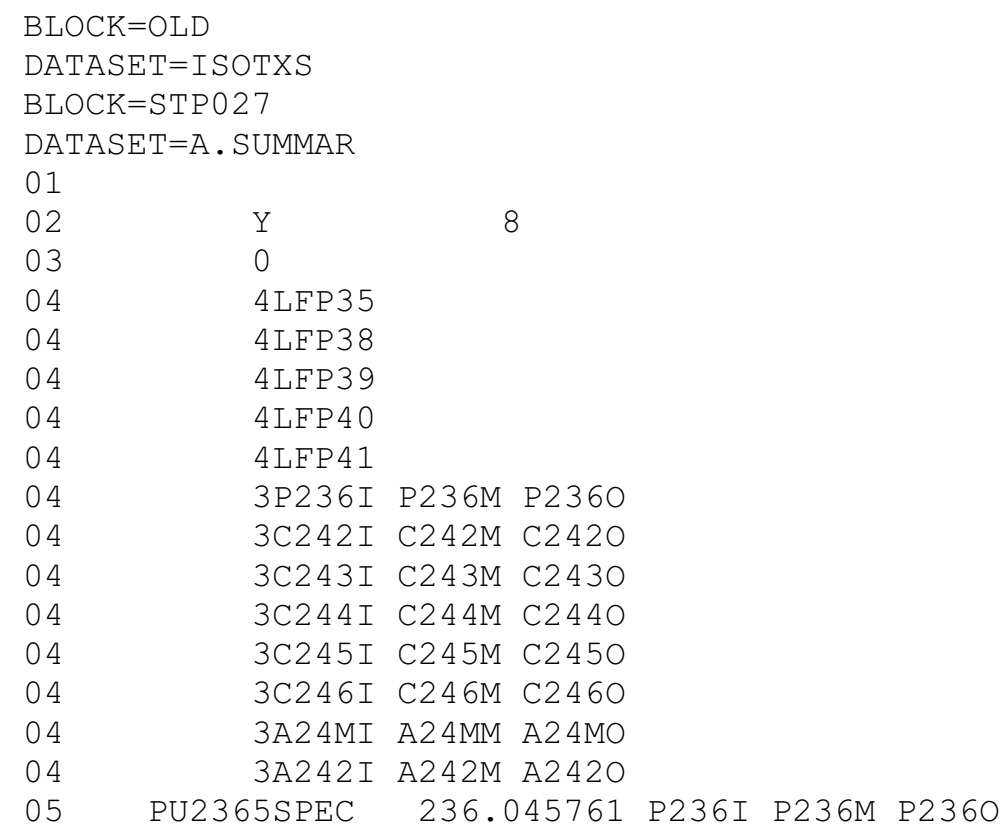

Figure A.IX.1: REBUS Equilibrium Model, CR=0.77. 


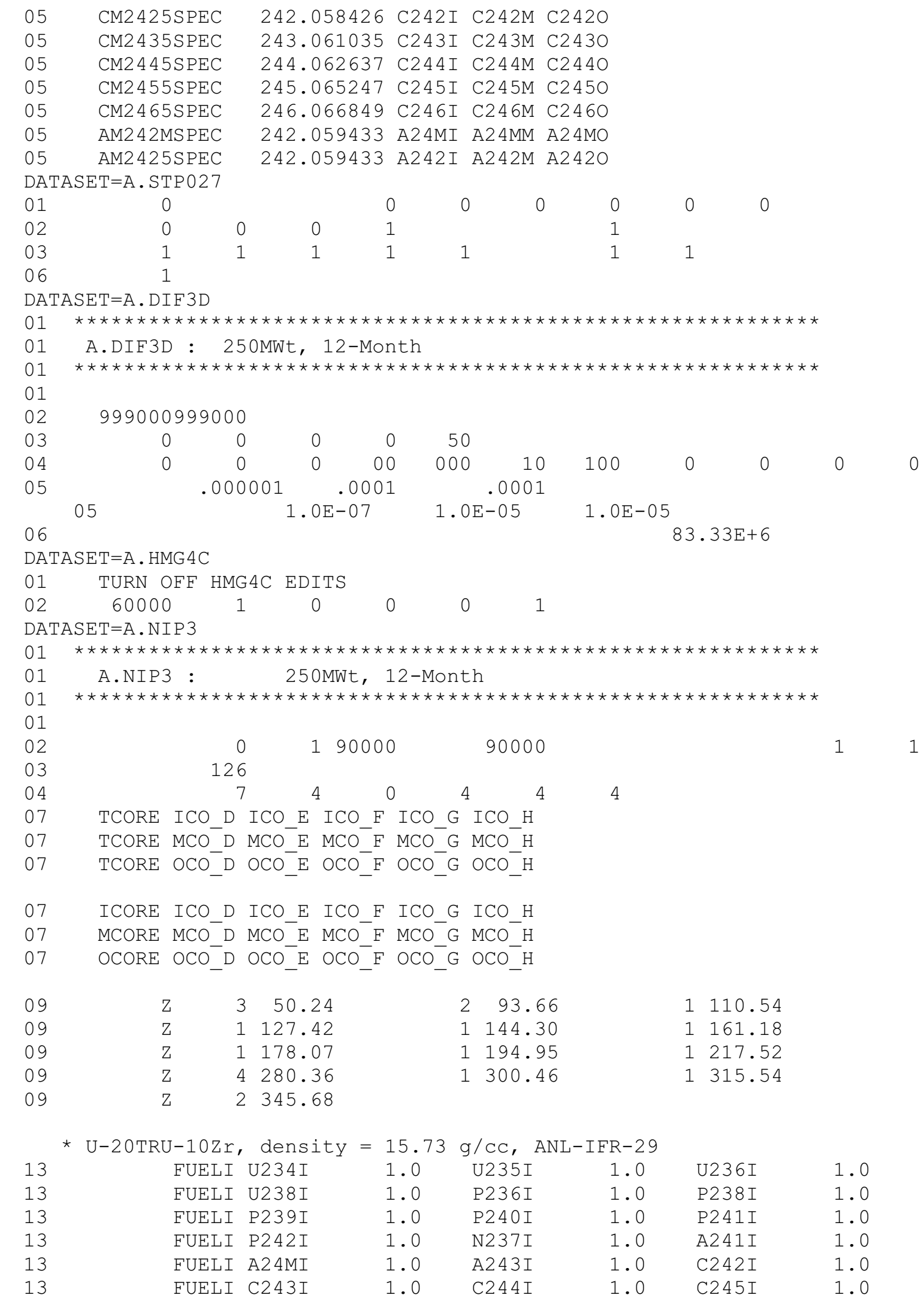

Figure A. IX.1: REBUS Equilibrium Model, $C R=0.77$, cont. 


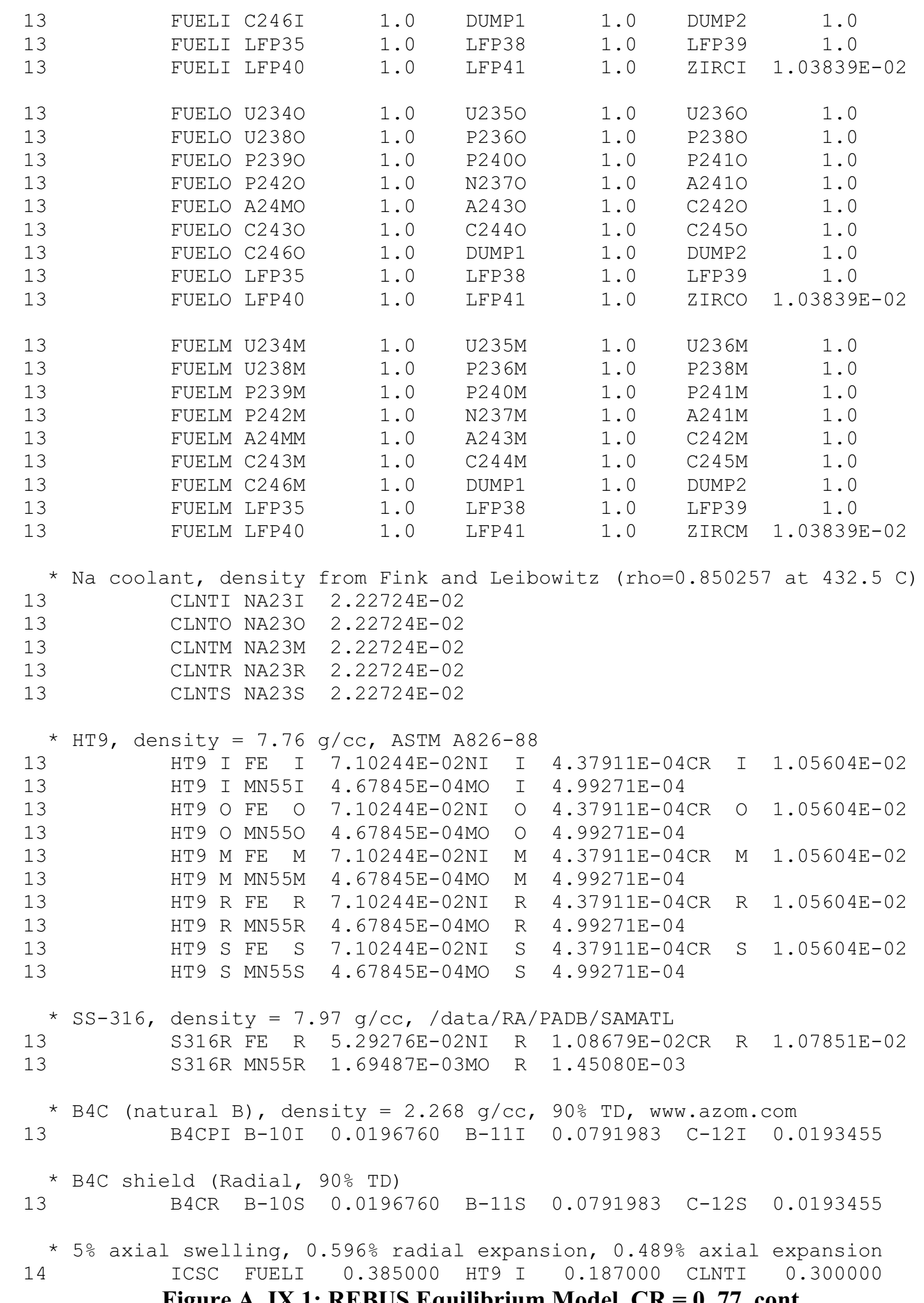

Figure A. IX.1: REBUS Equilibrium Model, $C R=0.77$, cont. 


\begin{tabular}{|c|c|c|c|c|c|c|c|c|}
\hline 14 & & OCSC & FUELO & 0.385000 & HT9 0 & 0.187000 & CLNTO & 0.300000 \\
\hline 14 & & MCSC & FUELM & 0.385000 & HT9 M & 0.187000 & CLNTM & 0.300000 \\
\hline 14 & & LPSC & S316R & 0.3 & CLNTR & 0.7 & & \\
\hline 14 & & LRSC & HT9 R & 0.667897 & CLNTR & 0.320813 & & \\
\hline 14 & & UPSC1 & HT9 R & 0.227980 & CLNTR & 0.768166 & & \\
\hline 14 & & UPSC & HT9 R & 0.227980 & CLNTR & 0.320813 & & \\
\hline 14 & & USSC & HT 9 R & 0.667897 & CLNTR & 0.320813 & & \\
\hline * & pellet & volume & fracti & for $B 4 C$ & with th & mal expar & sion & \\
\hline 14 & & CRBSC & HT9 I & 0.263966 & CLNTI & 0.366908 & $\mathrm{~B} 4 \mathrm{CPI}$ & 0.308300 \\
\hline 14 & & CRCSC & HT9 I & 0.076960 & CLNTI & 0.921739 & & \\
\hline 14 & & CRFSC & HT9 I & 0.247787 & CLNTI & 0.748024 & & \\
\hline 14 & & CRPSC & HT9 $R$ & 0.263966 & CLNTR & 0.366908 & & \\
\hline 14 & & CRDSC & HT9 R & 0.247787 & CLNTR & 0.748024 & & \\
\hline * & reflecto & or and & shielc & & & & & \\
\hline 14 & & REFSC & HT9 R & 0.828951 & CLNTR & 0.157036 & & \\
\hline 14 & & $\mathrm{RS} 2 \mathrm{SC}$ & HT9 $\mathrm{S}$ & 0.299011 & CLNTS & 0.173203 & B 4 CR & 0.421138 \\
\hline 14 & & BRSC & S316R & 0.062 & CLNTS & 0.938 & & \\
\hline * & primary & compos & sitions & & & & & \\
\hline 14 & & ICPC & ICSC & 1.0 & & & & \\
\hline 14 & & OCPC & OCSC & 1.0 & & & & \\
\hline 14 & & $\mathrm{MCPC}$ & $\mathrm{MCSC}$ & 1.0 & & & & \\
\hline 14 & & LPPC & LPSC & 1.0 & & & & \\
\hline 14 & & LRPC & LRSC & 1.0 & & & & \\
\hline 14 & & UPPC1 & UPSC1 & 1.0 & & & & \\
\hline 14 & & UPPC & UPSC & 1.0 & & & & \\
\hline 14 & & USPC & USSC & 1.0 & & & & \\
\hline 14 & & BRPC & BRSC & 1.0 & & & & \\
\hline 14 & & CRBPC & CRBSC & 1.0 & & & & \\
\hline 14 & & CRCPC & CRCSC & 1.0 & & & & \\
\hline 14 & & CRFPC & CRFSC & 1.0 & & & & \\
\hline 14 & & CRPPC & CRPSC & 1.0 & & & & \\
\hline 14 & & CRDPC & CRDSC & 1.0 & & & & \\
\hline 14 & & REFPC & REFSC & 1.0 & & & & \\
\hline 14 & & RS2PC & RS2SC & 1.0 & & & & \\
\hline 15 & LPPC & $\mathrm{CR} \quad \mathrm{A}$ & & & & & & \\
\hline 15 & LRPC & $\mathrm{CR}^{-} \mathrm{B}$ & & & & & & \\
\hline 15 & CRCPC & $\mathrm{CR}-\mathrm{C}$ & & & & & & \\
\hline 15 & CRFPC & $\mathrm{CR}_{-\mathrm{G}}$ & & & & & & \\
\hline 15 & CRBPC & $\mathrm{CR}-\mathrm{H}$ & & & & & & \\
\hline 15 & CRPPC & $\mathrm{CR} I$ & & & & & & \\
\hline 15 & CRDPC & $\mathrm{CR} \mathrm{J}$ & & & & & & \\
\hline 15 & USPC & $\mathrm{CR}^{-} \mathrm{K}$ & & & & & & \\
\hline 15 & LPPC & ICO A & & & & & & \\
\hline 15 & LPPC & OCO_A & & & & & & \\
\hline 15 & LPPC & $\mathrm{MCO}_{-}^{-} \mathrm{A}$ & & & & & & \\
\hline 15 & LRPC & ICO B & & & & & & \\
\hline 15 & LRPC & $\mathrm{OCO}^{-} \mathrm{B}$ & & & & & & \\
\hline 15 & LRPC & $\mathrm{MCO}^{-} \mathrm{B}$ & & & & & & \\
\hline
\end{tabular}

Figure A. IX.1: REBUS Equilibrium Model, $C R=0.77$, cont. 


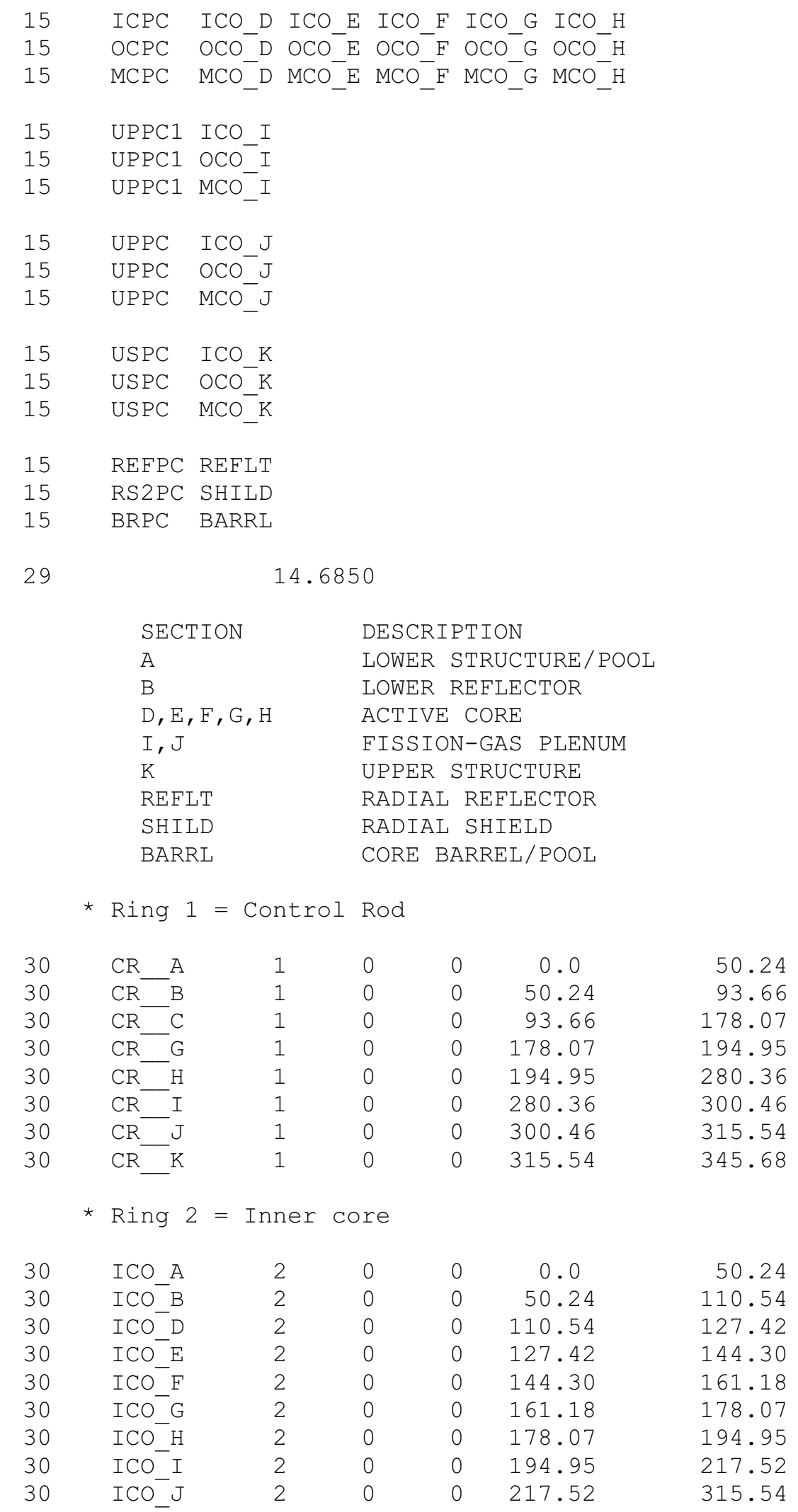

Figure A.IX.1: REBUS Equilibrium Model, $C R=0.77$, cont. 


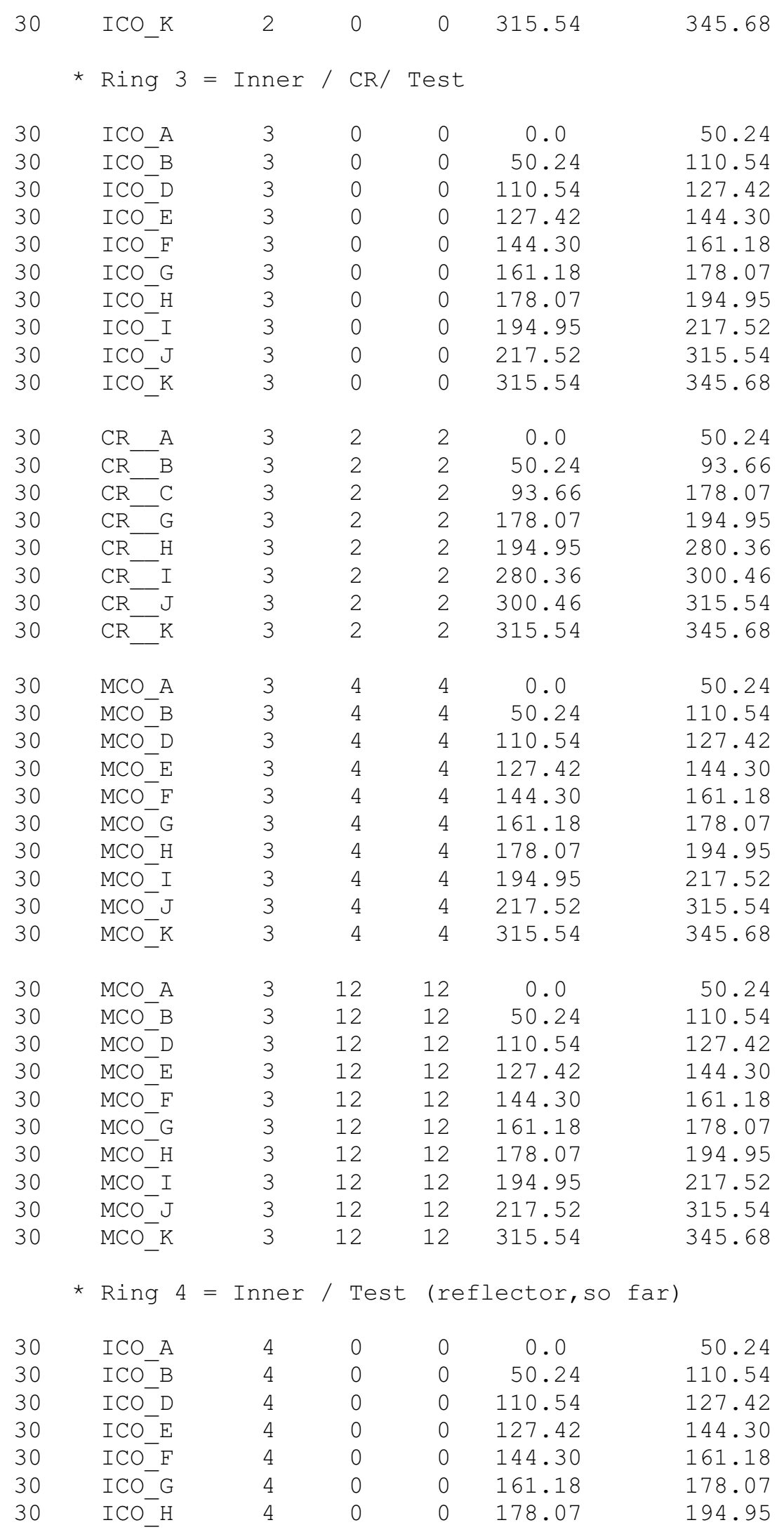

Figure A.IX.1: REBUS Equilibrium Model, $C R=0.77$, cont. 


\begin{tabular}{|c|c|c|c|c|c|c|}
\hline 30 & $\mathrm{ICO} I$ & 4 & 0 & 0 & 194.95 & 217.52 \\
\hline 30 & $\mathrm{ICO}_{-}^{-} \mathrm{J}$ & 4 & 0 & 0 & 217.52 & 315.54 \\
\hline 30 & $\mathrm{ICO}^{-} \mathrm{K}$ & 4 & 0 & 0 & 315.54 & 345.68 \\
\hline 30 & $\mathrm{MCO} \mathrm{A}$ & 4 & 1 & 1 & 0.0 & 50.24 \\
\hline 30 & $\mathrm{MCO}^{-} \mathrm{B}$ & 4 & 1 & 1 & 50.24 & 110.54 \\
\hline 30 & $\mathrm{MCO}^{-} \mathrm{D}$ & 4 & 1 & 1 & 110.54 & 127.42 \\
\hline 30 & $\mathrm{MCO}^{-} \mathrm{E}$ & 4 & 1 & 1 & 127.42 & 144.30 \\
\hline 30 & $\mathrm{MCO}^{-} \mathrm{F}$ & 4 & 1 & 1 & 144.30 & 161.18 \\
\hline 30 & $\mathrm{MCO}_{-}^{-} \mathrm{G}$ & 4 & 1 & 1 & 161.18 & 178.07 \\
\hline 30 & $\mathrm{MCO}_{-}^{-} \mathrm{H}$ & 4 & 1 & 1 & 178.07 & 194.95 \\
\hline 30 & $\mathrm{MCO}_{-}^{-} \mathrm{I}$ & 4 & 1 & 1 & 194.95 & 217.52 \\
\hline 30 & $\mathrm{MCO}_{-}^{-} \mathrm{J}$ & 4 & 1 & 1 & 217.52 & 315.54 \\
\hline 30 & $\mathrm{MCO}_{-}^{-} \mathrm{K}$ & 4 & 1 & 1 & 315.54 & 345.68 \\
\hline 30 & REFLT & 4 & 4 & 4 & 0.0 & 345.68 \\
\hline & Ring 5 & $=$ Outer & / $\mathrm{CR}$ & & & \\
\hline 30 & $\mathrm{OCO} A$ & 5 & 0 & 0 & 0.0 & 50.24 \\
\hline 30 & $\mathrm{OCO}^{-} \mathrm{B}$ & 5 & 0 & 0 & 50.24 & 110.54 \\
\hline 30 & $\mathrm{OCO}_{-}^{-} \mathrm{D}$ & 5 & 0 & 0 & 110.54 & 127.42 \\
\hline 30 & $\mathrm{OCO}_{-}^{-} \mathrm{E}$ & 5 & 0 & 0 & 127.42 & 144.30 \\
\hline 30 & $\mathrm{OCO}_{-}^{-} \mathrm{F}$ & 5 & 0 & 0 & 144.30 & 161.18 \\
\hline 30 & $\mathrm{OCO}_{-}^{-} \mathrm{G}$ & 5 & 0 & 0 & 161.18 & 178.07 \\
\hline 30 & $\mathrm{OCO}_{-}^{-} \mathrm{H}$ & 5 & 0 & 0 & 178.07 & 194.95 \\
\hline 30 & $\mathrm{OCO}_{-}^{-} \mathrm{I}$ & 5 & 0 & 0 & 194.95 & 217.52 \\
\hline 30 & $\mathrm{OCO}_{-}^{-} \mathrm{J}$ & 5 & 0 & 0 & 217.52 & 315.54 \\
\hline 30 & $\mathrm{OCO}_{-}^{-} \mathrm{K}$ & 5 & 0 & 0 & 315.54 & 345.68 \\
\hline 30 & $\mathrm{CR} \quad \mathrm{A}$ & 5 & 3 & 3 & 0.0 & 50.24 \\
\hline 30 & $\mathrm{CR} \quad \mathrm{B}$ & 5 & 3 & 3 & 50.24 & 93.66 \\
\hline 30 & $\mathrm{CR} \quad \mathrm{C}$ & 5 & 3 & 3 & 93.66 & 178.07 \\
\hline 30 & $\mathrm{CR} G \mathrm{G}$ & 5 & 3 & 3 & 178.07 & 194.95 \\
\hline 30 & $\mathrm{CR}-\mathrm{H}$ & 5 & 3 & 3 & 194.95 & 280.36 \\
\hline 30 & $\mathrm{CR} I$ & 5 & 3 & 3 & 280.36 & 300.46 \\
\hline 30 & $\mathrm{CR} \mathrm{J}$ & 5 & 3 & 3 & 300.46 & 315.54 \\
\hline 30 & $\mathrm{CR}-\mathrm{K}$ & 5 & 3 & 3 & 315.54 & 345.68 \\
\hline 30 & $\mathrm{CR} \quad \mathrm{A}$ & 5 & 7 & 7 & 0.0 & 50.24 \\
\hline 30 & $\mathrm{CR} \quad \mathrm{B}$ & 5 & 7 & 7 & 50.24 & 93.66 \\
\hline 30 & $\mathrm{CR}-\mathrm{C}$ & 5 & 7 & 7 & 93.66 & 178.07 \\
\hline 30 & $\mathrm{CR} G$ & 5 & 7 & 7 & 178.07 & 194.95 \\
\hline 30 & $\mathrm{CR}-\mathrm{H}$ & 5 & 7 & 7 & 194.95 & 280.36 \\
\hline 30 & $\mathrm{CR} I$ & 5 & 7 & 7 & 280.36 & 300.46 \\
\hline 30 & $\mathrm{CR} \mathrm{J}$ & 5 & 7 & 7 & 300.46 & 315.54 \\
\hline 30 & $\mathrm{CR}-\mathrm{K}$ & 5 & 7 & 7 & 315.54 & 345.68 \\
\hline 30 & $\mathrm{CR} \quad \mathrm{A}$ & 5 & 23 & 23 & 0.0 & 50.24 \\
\hline 30 & $\mathrm{CR} B \mathrm{~B}$ & 5 & 23 & 23 & 50.24 & 93.66 \\
\hline 30 & $\mathrm{CR}-\mathrm{C}$ & 5 & 23 & 23 & 93.66 & 178.07 \\
\hline 30 & $\mathrm{CR} G$ & 5 & 23 & 23 & 178.07 & 194.95 \\
\hline 30 & $\mathrm{CR}-\mathrm{H}$ & 5 & 23 & 23 & 194.95 & 280.36 \\
\hline 30 & $\mathrm{CR} I$ & 5 & 23 & 23 & 280.36 & 300.46 \\
\hline 30 & $\mathrm{CR} \mathrm{J}$ & 5 & 23 & 23 & 300.46 & 315.54 \\
\hline
\end{tabular}

Figure A.IX.1: REBUS Equilibrium Model, $C R=0.77$, cont. 


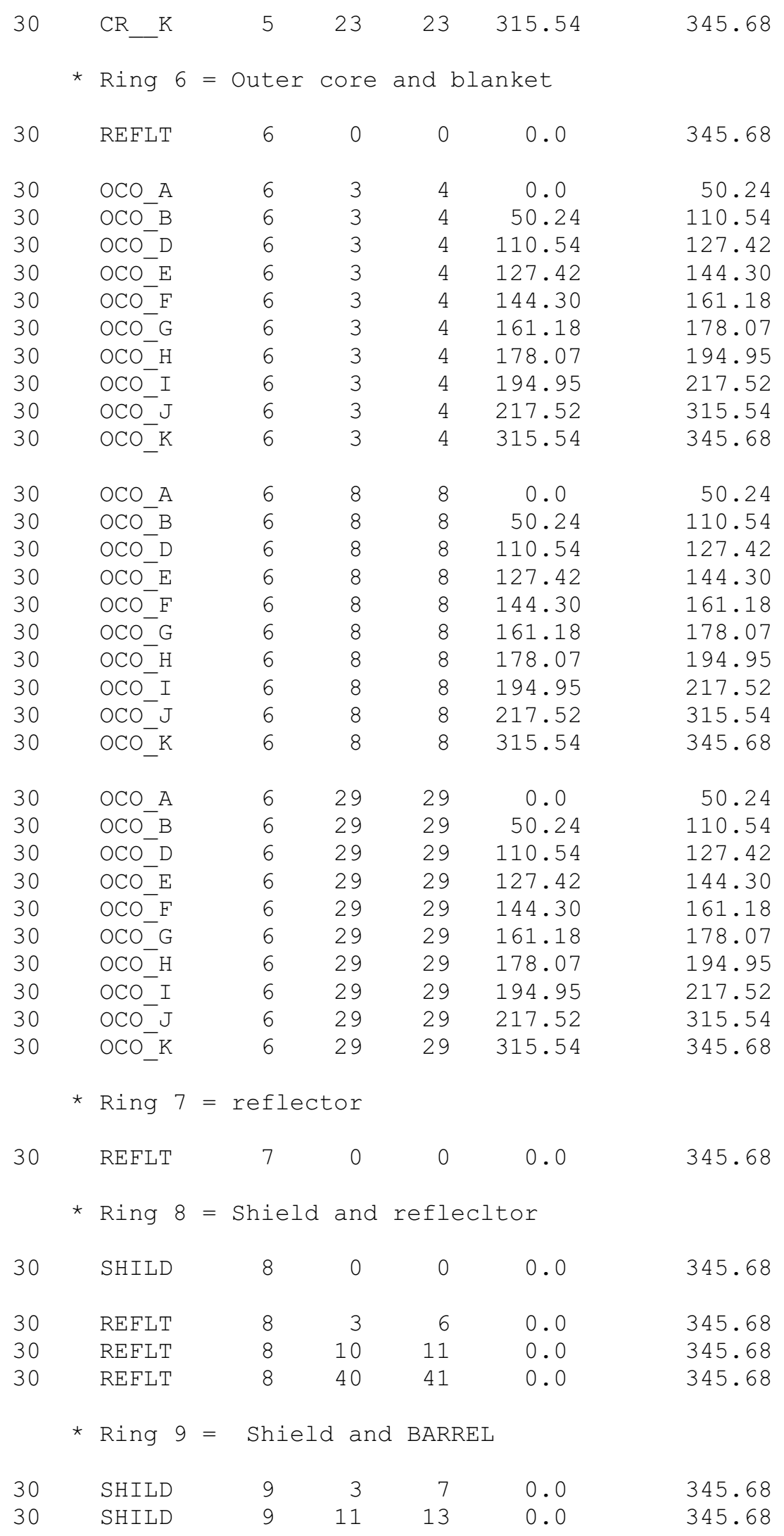

Figure A.IX.1: REBUS Equilibrium Model, $C R=0.77$, cont. 


\begin{tabular}{|c|c|c|c|c|c|c|c|}
\hline 30 & SHILD & 9 & 47 & 0.0 & 345.68 & & \\
\hline 30 & BARRL & 9 & 2 & 0.0 & 345.68 & & \\
\hline 30 & BARRL & 9 & 8 & 0.0 & 345.68 & & \\
\hline 30 & BARRL & 9 & 10 & 0.0 & 345.68 & & \\
\hline 30 & BARRL & 9 & 48 & 0.0 & 345.68 & & \\
\hline \multicolumn{8}{|c|}{ DATASET $=$ A. BURN } \\
\hline 01 & \multicolumn{7}{|c|}{ 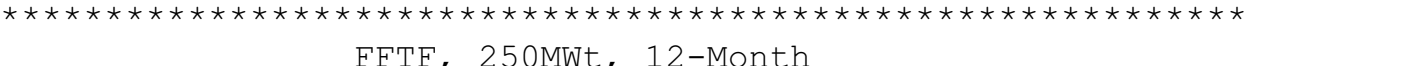 } \\
\hline 01 & \multicolumn{7}{|c|}{ FFTF, 250MWt, 12-Month } \\
\hline 01 & \multirow{2}{*}{\multicolumn{7}{|c|}{ 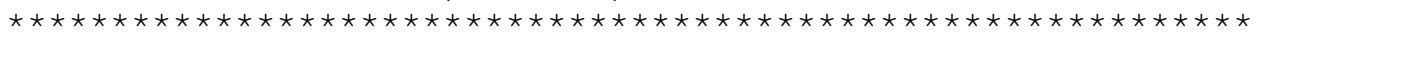 }} \\
\hline 01 & & & & & & & \\
\hline 02 & \multicolumn{2}{|c|}{999000} & 0.001 & 0.001 & 0.0001 & 2 & 1 \\
\hline 03 & 0 & 0.0 & & 0.0 & $121.7 \quad 1.00$ & 1 & 0 \\
\hline 04 & & 1.0000 & 0.001 & 1.0 & 0.170 & 0.210 & \\
\hline 06 & & 0.5 & & & & & \\
\hline 09 & $\mathrm{U}-234$ & $1 \mathrm{U}-235$ & & & & & \\
\hline 09 & $U-234$ & $2 \operatorname{LFP} 35$ & & & & & \\
\hline 09 & $U-234$ & 5DUMP1 & & & & & \\
\hline 09 & $U-234$ & 8DUMP1 & & & & & \\
\hline 25 & $U-234$ & 8DUMP 1 & 8.9 & $3-14$ & & & \\
\hline 09 & $U-235$ & $1 \mathrm{U}-236$ & & & & & \\
\hline 09 & $U-235$ & 2LFP3 5 & & & & & \\
\hline 09 & $U-235$ & $5 U-234$ & & & & & \\
\hline 09 & $U-235$ & 8DUMP1 & & & & & \\
\hline 25 & $U-235$ & 8DUMP1 & 3.1 & -17 & & & \\
\hline 09 & $U-236$ & $1 \mathrm{NP} 237$ & & & & & \\
\hline 09 & $U-236$ & 2LFP3 5 & & & & & \\
\hline 09 & $U-236$ & $5 U-235$ & & & & & \\
\hline 09 & $U-236$ & 8DUMP1 & & & & & \\
\hline 25 & $U-236$ & 8DUMP 1 & 9.3 & $9-16$ & & & \\
\hline 09 & $U-238$ & 1 PU239 & & & & & \\
\hline 09 & $U-238$ & 2LFP3 8 & & & & & \\
\hline 09 & $U-238$ & $5 \mathrm{NP} 237$ & & & & & \\
\hline 09 & $U-238$ & 8DUMP1 & & & & & \\
\hline 25 & $U-238$ & 8DUMP1 & 4.9 & $5-18$ & & & \\
\hline 09 & NP237 & 1 PU23 8 & & & & & \\
\hline 09 & NP237 & 2LFP3 8 & & & & & \\
\hline 09 & NP237 & 5 PU23 6 & & $346 \mathrm{U}-236$ & 0.374 DUMP 1 & & 0.28 \\
\hline 09 & NP237 & 8DUMP1 & & & & & \\
\hline 25 & NP237 & 8DUMP1 & 1.0 & $5-14$ & & & \\
\hline 09 & PU236 & $1 \mathrm{NP} 237$ & & & & & \\
\hline 09 & PU236 & 2LFP3 5 & & & & & \\
\hline 09 & PU236 & 5DUMP1 & & & & & \\
\hline 09 & PU236 & 8DUMP1 & & & & & \\
\hline 25 & PU236 & 8DUMP1 & 7.7 & $3-09$ & & & \\
\hline 09 & PU238 & 1 PU239 & & & & & \\
\hline 09 & PU238 & 2LFP3 8 & & & & & \\
\hline 09 & PU238 & $5 \mathrm{NP} 237$ & & & & & \\
\hline 09 & PU238 & $8 \mathrm{U}-234$ & & & & & \\
\hline 25 & PU238 & $8 \mathrm{U}-234$ & 2.5 & $3-10$ & & & \\
\hline 09 & PU239 & 1 PU2 40 & & & & & \\
\hline 09 & PU239 & $2 \operatorname{LFP} 39$ & & & & & \\
\hline 09 & PU239 & 5 PU23 8 & & & & & \\
\hline
\end{tabular}

Figure A.IX.1: REBUS Equilibrium Model, $C R=0.77$, cont. 


\begin{tabular}{|c|c|c|c|c|c|}
\hline 09 & PU239 & $8 U-235$ & & & \\
\hline 25 & PU239 & $8 U-235$ & $9.109-13$ & & \\
\hline 09 & PU24 0 & 1 PU2 41 & & & \\
\hline 09 & PU24 0 & $2 \mathrm{LEP} 40$ & & & \\
\hline 09 & PU2 40 & 5PU239 & & & \\
\hline 09 & PU240 & $8 U-236$ & & & \\
\hline 25 & PU240 & $8 U-236$ & $3.353-12$ & & \\
\hline 09 & PU241 & 1 PU2 42 & & & \\
\hline 09 & PU241 & $2 \mathrm{LFP} 41$ & & & \\
\hline 09 & PU241 & 5 PU2 40 & & & \\
\hline 09 & PU241 & 6AM2 41 & & & \\
\hline 25 & PU241 & $6 \mathrm{AM} 241$ & $1.494-09$ & & \\
\hline 09 & PU242 & 1AM2 43 & & & \\
\hline 09 & PU242 & $2 \mathrm{LFP} 41$ & & & \\
\hline 09 & PU242 & 5PU2 41 & & & \\
\hline 09 & PU242 & $8 \mathrm{U}-238$ & & & \\
\hline 25 & PU242 & $8 U-238$ & $5.833-14$ & & \\
\hline 09 & AM2 41 & 1CM2 42 & $0.66 \mathrm{AM} 242$ & 0.20 PU2 42 & 0.14 \\
\hline 09 & AM2 41 & 2LFP 41 & & & \\
\hline 09 & AM2 41 & 5 PU2 40 & & & \\
\hline 09 & AM2 41 & 8NP237 & & & \\
\hline 25 & AM2 41 & 8NP237 & $5.081-11$ & & \\
\hline 09 & AM2 42 & 1AM2 43 & & & \\
\hline 09 & AM2 42 & $2 \mathrm{LFP} 41$ & & & \\
\hline 09 & AM2 42 & 5AM2 41 & & & \\
\hline 09 & AM2 42 & $6 \mathrm{CM} 242$ & & & \\
\hline 25 & AM2 42 & $6 \mathrm{CM} 242$ & $1.189-10$ & & \\
\hline 09 & AM2 42 & 7 PU2 42 & & & \\
\hline 25 & AM2 42 & 7 PU2 42 & $2.487-11$ & & \\
\hline 09 & AM2 42 & 8PU238 & & & \\
\hline 25 & AM2 42 & $8 \mathrm{PU} 238$ & $7.225-13$ & & \\
\hline 09 & AM2 43 & $1 \mathrm{CM} 244$ & & & \\
\hline 09 & AM2 43 & $2 \mathrm{LFP} 41$ & & & \\
\hline 09 & AM2 43 & $5 \mathrm{AM} 242$ & $0.500 \mathrm{PU} 242$ & $0.086 \mathrm{CM} 242$ & 0.414 \\
\hline 09 & AM2 43 & 8PU239 & & & \\
\hline 25 & AM2 43 & 8PU239 & $2.976-12$ & & \\
\hline 09 & CM242 & $1 \mathrm{CM} 243$ & & & \\
\hline 09 & CM242 & $2 \mathrm{LFP} 41$ & & & \\
\hline 09 & CM242 & 5AM2 41 & $0.99 \mathrm{NP} 237$ & 0.01 & \\
\hline 09 & CM242 & 8PU238 & & & \\
\hline 25 & CM242 & 8PU238 & $4.924-08$ & & \\
\hline 09 & CM243 & $1 \mathrm{CM} 244$ & & & \\
\hline 09 & CM243 & $2 \mathrm{LFP} 41$ & & & \\
\hline 09 & CM2 43 & $5 \mathrm{CM} 242$ & & & \\
\hline 09 & CM243 & 7AM2 43 & & & \\
\hline 25 & CM243 & 7AM2 43 & $2.003-12$ & & \\
\hline 09 & CM243 & 8PU239 & & & \\
\hline 25 & CM243 & 8PU239 & $7.685-10$ & & \\
\hline 09 & CM2 44 & $1 \mathrm{CM} 245$ & & & \\
\hline 09 & CM2 44 & $2 \mathrm{LFP} 41$ & & & \\
\hline 09 & CM2 44 & $5 \mathrm{CM} 243$ & & & \\
\hline 09 & CM2 44 & 8PU2 40 & & & \\
\hline 25 & CM2 44 & 8PU2 40 & $1.213-09$ & & \\
\hline 09 & CM2 45 & $1 \mathrm{CM} 246$ & & & \\
\hline 09 & CM245 & 2LFP41 & & & \\
\hline
\end{tabular}

Figure A.IX.1: REBUS Equilibrium Model, $C R=0.77$, cont. 


\begin{tabular}{|c|c|c|c|c|c|c|c|}
\hline 09 & CM24 5 & & 5CM2 44 & & & & \\
\hline 09 & CM2 45 & & 8PU241 & & & & \\
\hline 25 & CM24 5 & & 8PU2 41 & & $.592-12$ & & \\
\hline 09 & CM24 6 & & 1 DUMP2 & & & & \\
\hline 09 & CM24 6 & & $2 \mathrm{LFP} 41$ & & & & \\
\hline 09 & CM2 46 & & $5 \mathrm{CM} 245$ & & & & \\
\hline 09 & CM24 6 & & 8PU242 & & & & \\
\hline 25 & CM2 46 & & 8PU2 42 & & $.642-12$ & & \\
\hline 09 & LEP35 & & 0 & & & & \\
\hline 09 & LFP38 & & 0 & & & & \\
\hline 09 & LFP39 & & 0 & & & & \\
\hline 09 & LEP4 0 & & 0 & & & & \\
\hline 09 & LEP 41 & & 0 & & & & \\
\hline 09 & DUMP1 & & 0 & & & & \\
\hline 09 & DUMP2 & & 0 & & & & \\
\hline 10 & $\mathrm{U}-234$ & U234 I & $\mathrm{U} 234 \mathrm{M}$ & M U2340 & & & \\
\hline 10 & $\mathrm{U}-235$ & U235I & $\mathrm{U} 235 \mathrm{M}$ & M U2350 & & & \\
\hline 10 & $\mathrm{U}-236$ & U236I & $\mathrm{U} 236 \mathrm{M}$ & M U2360 & & & \\
\hline 10 & $\mathrm{U}-238$ & U238 I & $\mathrm{U} 238 \mathrm{M}$ & M U2380 & & & \\
\hline 10 & NP237 & N237I & $\mathrm{N} 237 \mathrm{M}$ & M N2370 & & & \\
\hline 10 & PU236 & P236I & P236M & M P2360 & & & \\
\hline 10 & PU238 & P238I & P238M & M P2380 & & & \\
\hline 10 & PU239 & P239I & P239M & M P2390 & & & \\
\hline 10 & PU240 & P240I & P2 $40 \mathrm{M}$ & M P2400 & & & \\
\hline 10 & PU241 & P241I & P2 $41 \mathrm{M}$ & M P2410 & & & \\
\hline 10 & PU242 & P242I & P242M & M P2420 & & & \\
\hline 10 & AM2 41 & A241I & A2 41M & M A2410 & & & \\
\hline 10 & AM2 42 & A24MI & $\mathrm{A} 24 \mathrm{MM}$ & $\mathrm{M}$ A $24 \mathrm{MO}$ & & & \\
\hline 10 & AM243 & A243I & A2 $43 \mathrm{M}$ & M A2430 & & & \\
\hline 10 & CM2 42 & C242I & $=\mathrm{C} 242 \mathrm{M}$ & M C2420 & & & \\
\hline 10 & CM243 & C243I & $=\mathrm{C} 243 \mathrm{M}$ & M C2430 & & & \\
\hline 10 & CM2 44 & C24 I I & $=\mathrm{C} 244 \mathrm{M}$ & M C2440 & & & \\
\hline 10 & CM2 45 & C245I & $=\mathrm{C} 245 \mathrm{M}$ & M C2450 & & & \\
\hline 10 & CM2 46 & C246I & $\mathrm{C} 246 \mathrm{M}$ & M C2460 & & & \\
\hline 11 & CPL1 & & 0 & 1ICSC & ICPC & $2 \mathrm{ICSC}$ & ICPC \\
\hline 11 & CPL1 & & 0 & $3 I C S C$ & ICPC & $4 I C S C$ & ICPC \\
\hline 11 & CPL1 & & 0 & 5ICSC & ICPC & $6 I C S C$ & ICPC \\
\hline 11 & CPL1 & & 0 & 7ICSC & ICPC & 8ICSC & ICPC \\
\hline 11 & CPL1 & & 0 & 9ICSC & ICPC & $10 I C S C$ & ICPC \\
\hline 11 & CPL1 & & 0 & $11 \mathrm{ICSC}$ & ICPC & 12 ICSC & ICPC \\
\hline 11 & CPL1 & & 0 & 13DISI & & & \\
\hline 11 & CPL2 & & 0 & $10 \mathrm{CSC}$ & OCPC & $20 \mathrm{CSC}$ & $\mathrm{OCPC}$ \\
\hline 11 & CPL2 & & 0 & $30 \mathrm{CSC}$ & OCPC & $4 \mathrm{OCSC}$ & $\mathrm{OCPC}$ \\
\hline 11 & CPL2 & & 0 & $50 \mathrm{CSC}$ & OCPC & $60 \mathrm{CSC}$ & $O C P C$ \\
\hline 11 & CPL2 & & 0 & 7OCSC & OCPC & $80 \mathrm{CSC}$ & $\mathrm{OCPC}$ \\
\hline 11 & CPL2 & & 0 & $90 \mathrm{CSC}$ & OCPC & $100 \mathrm{CSC}$ & $\mathrm{OCPC}$ \\
\hline 11 & CPL2 & & 0 & $110 \mathrm{CSC}$ & OCPC & $120 \mathrm{CSC}$ & $\mathrm{OCPC}$ \\
\hline 11 & CPL2 & & 0 & $130 \mathrm{CSC}$ & OCPC & $14 \mathrm{OCSC}$ & $\mathrm{OCPC}$ \\
\hline 11 & CPL2 & & 0 & $150 \mathrm{CSC}$ & OCPC & & \\
\hline 11 & CPL2 & & 0 & $16 \mathrm{DISO}$ & & & \\
\hline 11 & CPL3 & & 0 & $1 \mathrm{MCSC}$ & $\mathrm{MCPC}$ & $2 \mathrm{MCSC}$ & $\mathrm{MCPC}$ \\
\hline 11 & CPL3 & & 0 & 3MCSC & $\mathrm{MCPC}$ & $4 \mathrm{MCSC}$ & $\mathrm{MCPC}$ \\
\hline
\end{tabular}

Figure A.IX.1: REBUS Equilibrium Model, $C R=0.77$, cont. 


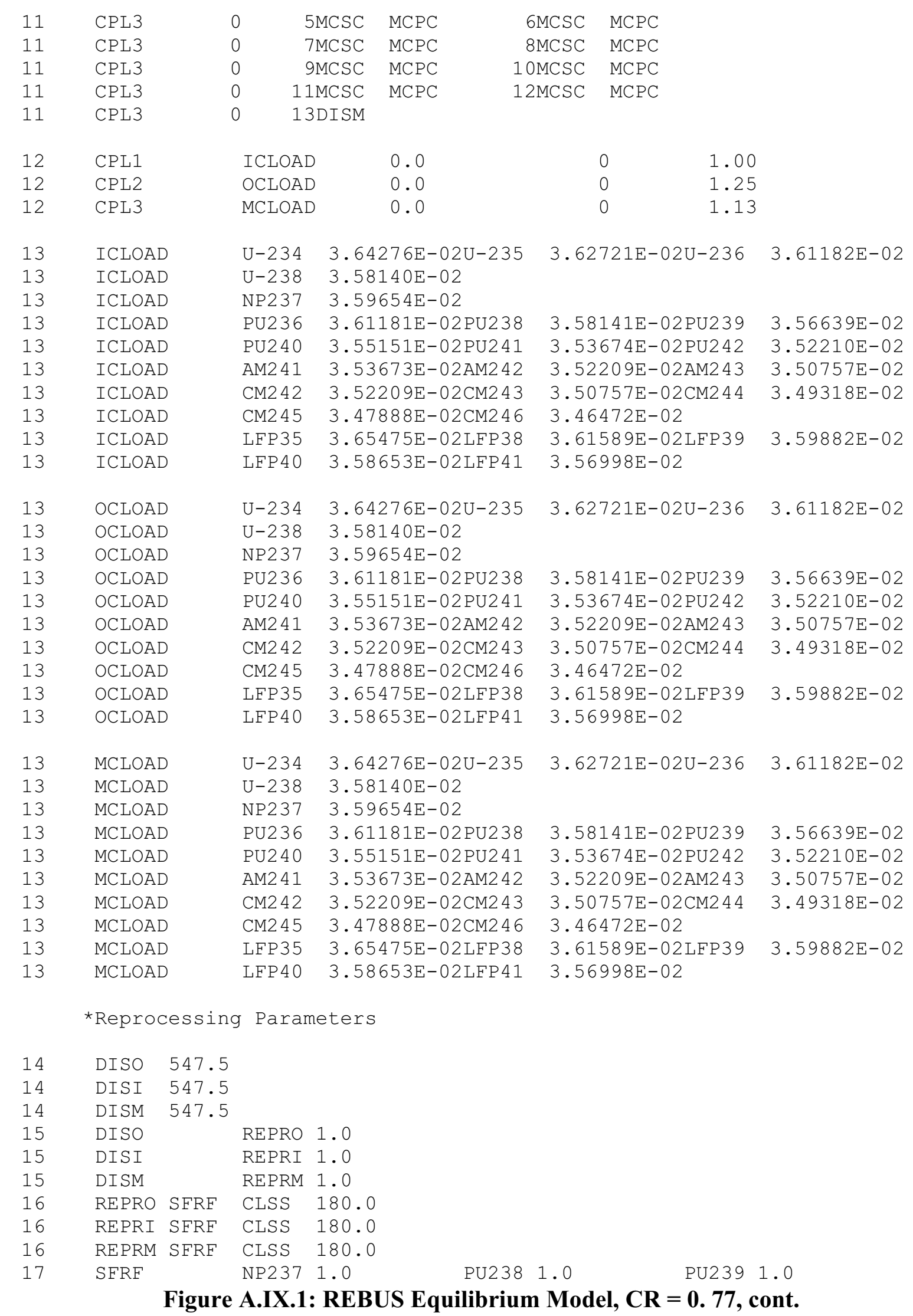




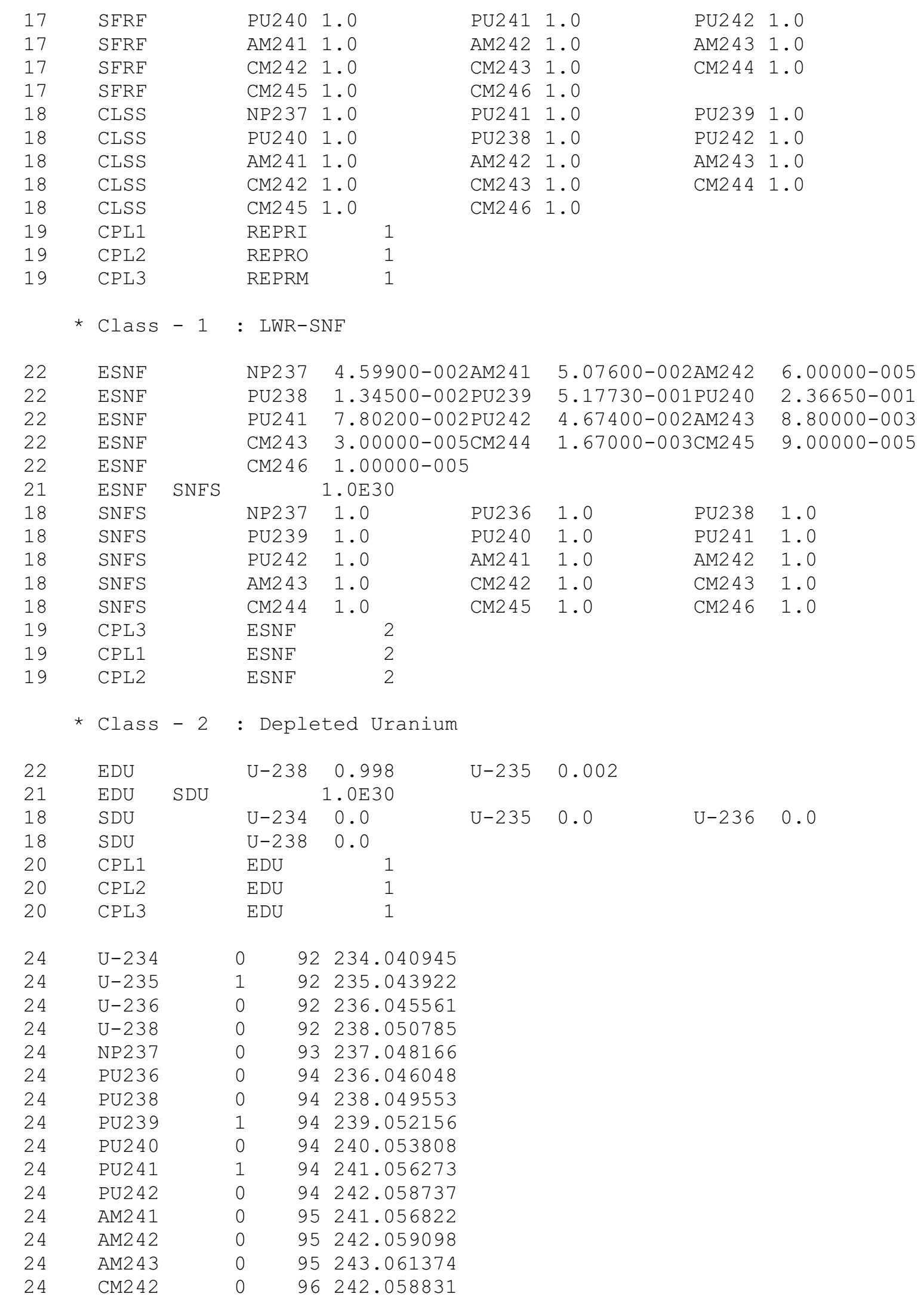

Figure A.IX.1: REBUS Equilibrium Model, $C R=0.77$, cont. 


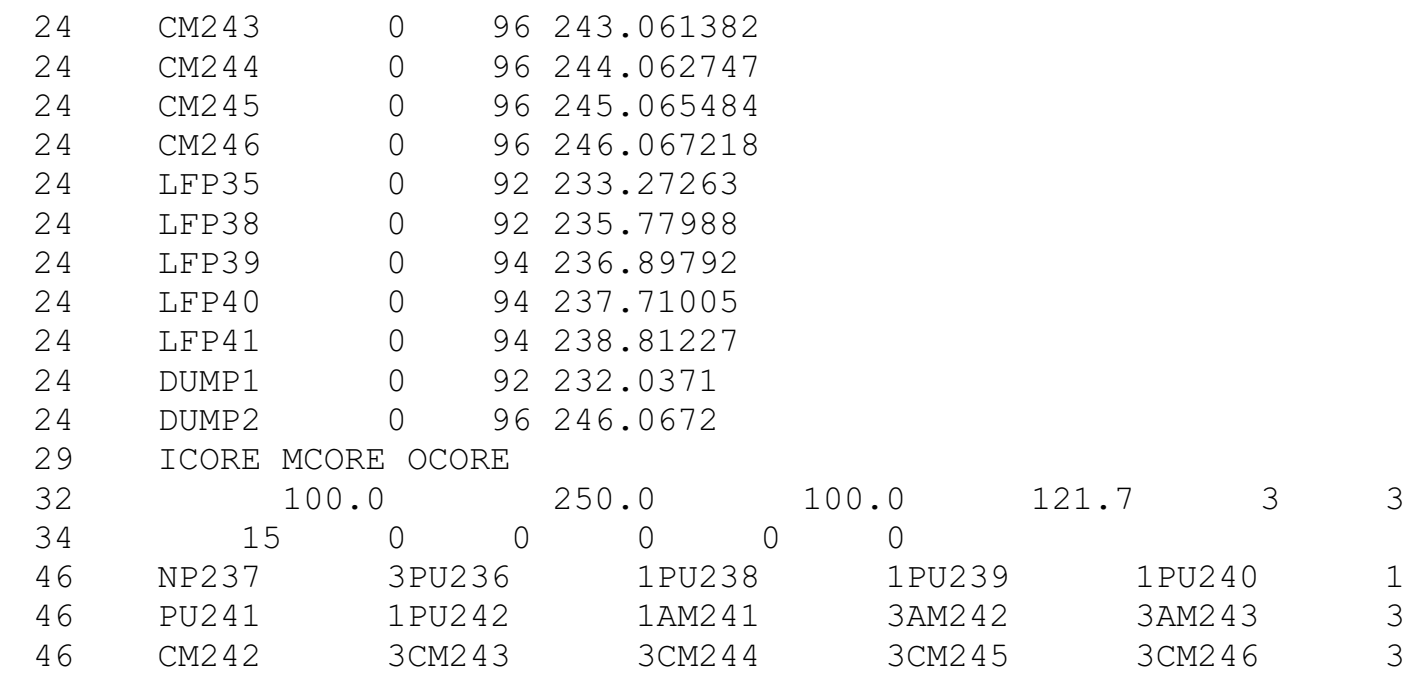

Figure A.IX.1: REBUS Equilibrium Model, $C R=0.77$, cont. 


\section{Appendix B: Graphical Verification of Model Linearity}

Presented below is a collection of 6 plots (Figures B.1a-1f) of selected nuclides showing that the general depletion model, namely ORIGEN, is essentially linear over the range of cross section perturbations applicable to this problem. The range used is cross sections perturbations from 0 to $+/-25 \%$ of the nominal value. Further, a corresponding set of 6 histograms, of the same nuclides, are shown representing the result of random sampling for that nuclides after implementing the foreword perturbation model (Figures B.2a-2f). The reader can see that these histograms resemble a Gaussian distribution, as the input cross sections were perturbed in a Gaussian distribution; a proof of linearity. 


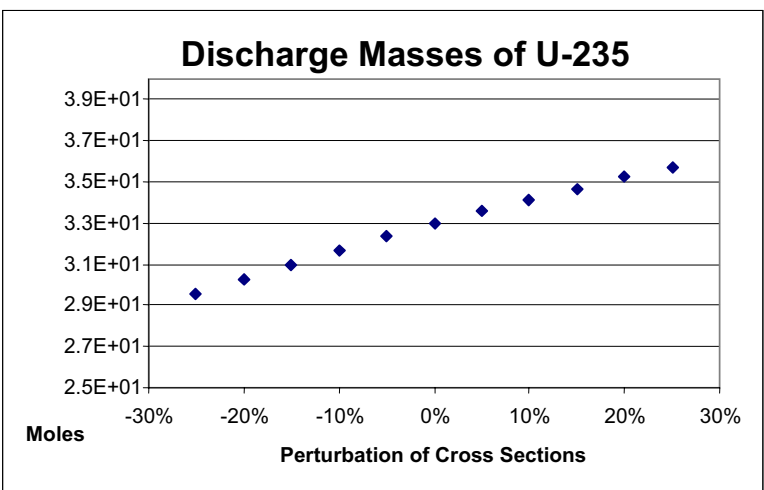

Figure B.1a: U-235 Linearity

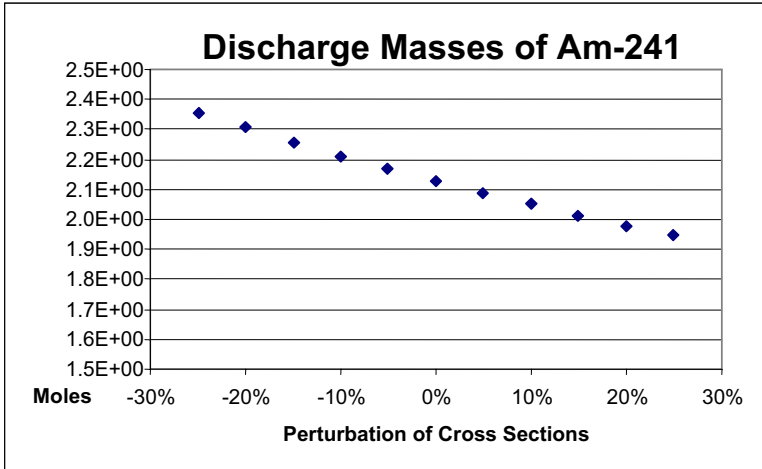

Figure B.1c: Am-241 Linearity

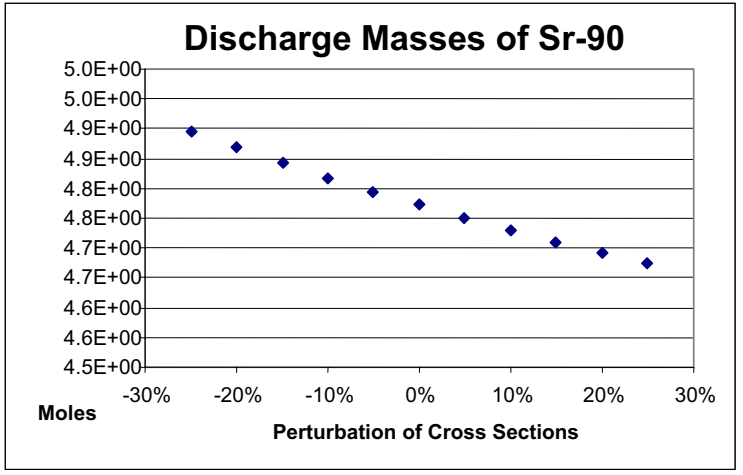

Figure B.1e: Sr-90 Linearity

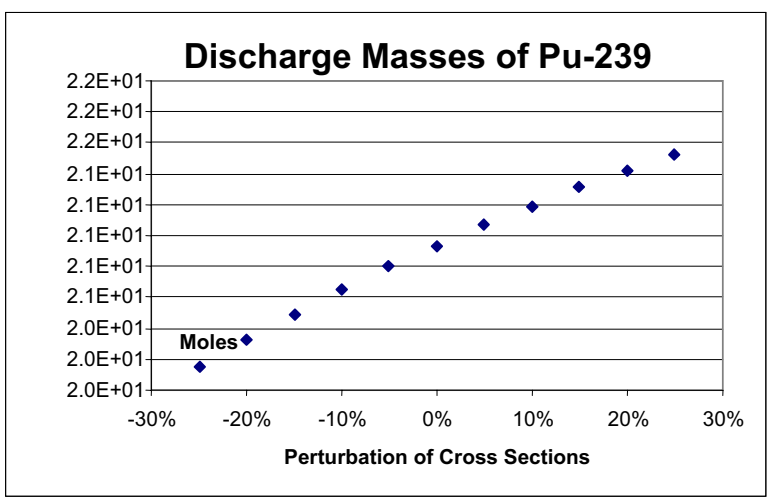

Figure B.1b: Pu-239 Linearity

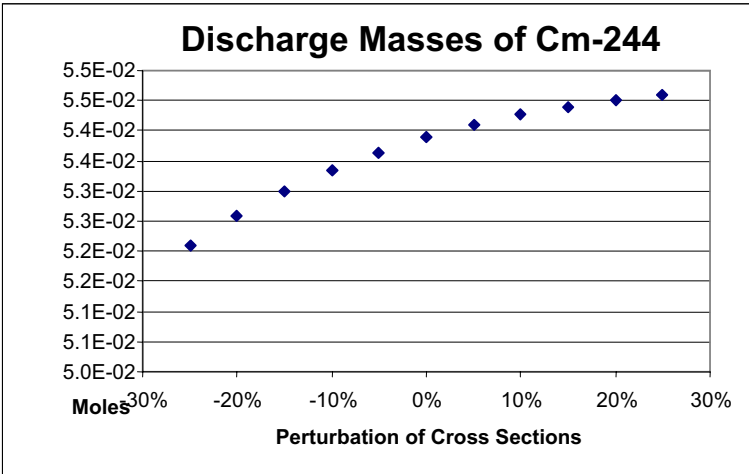

Figure B.1d: Cm-244 Linearity

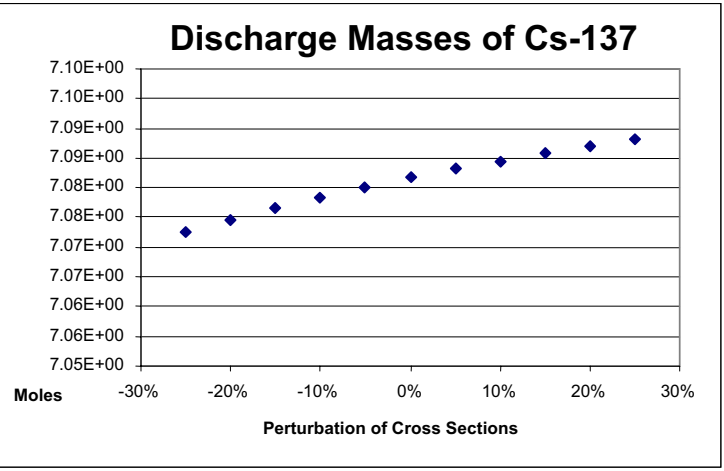

Figure B.1f: Cs-137 Linearity 


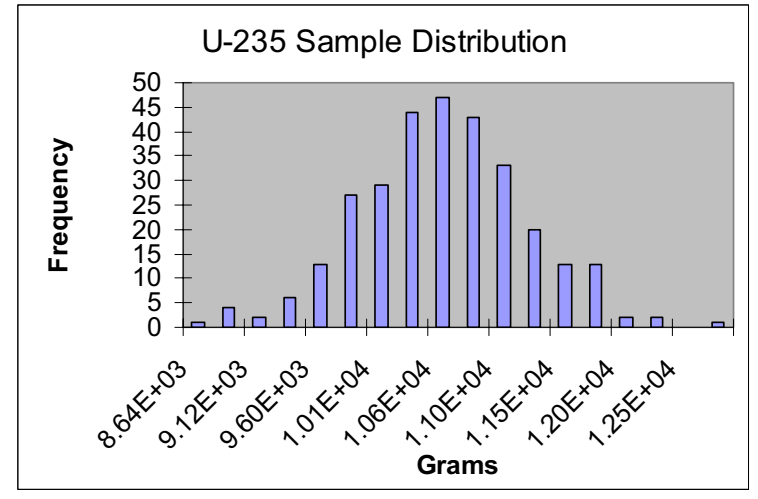

Figure B.2a: U-235 Distribution

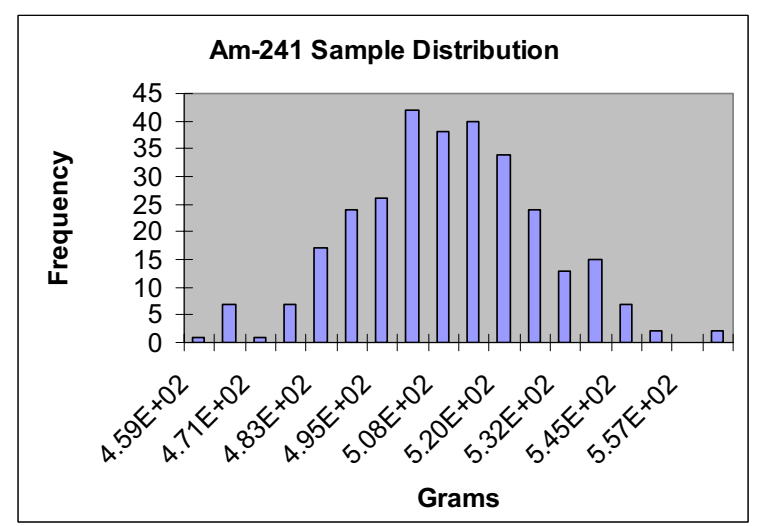

Figure B.2c: Am-241 Distribution

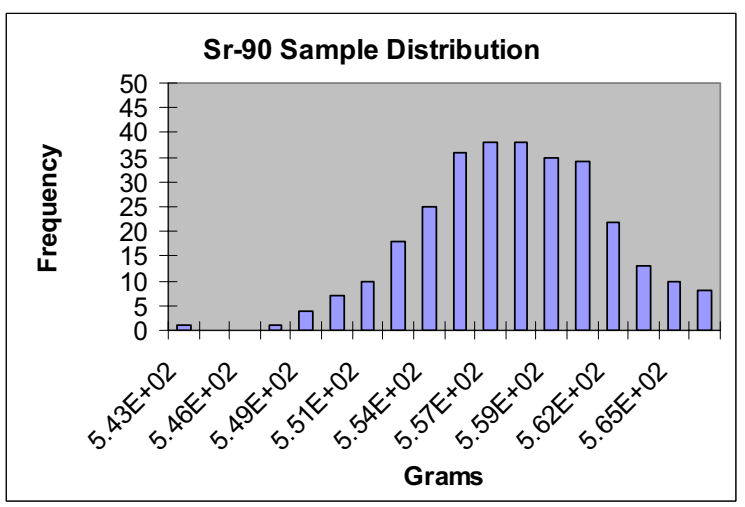

Figure B.2e: Sr-90 Distribution

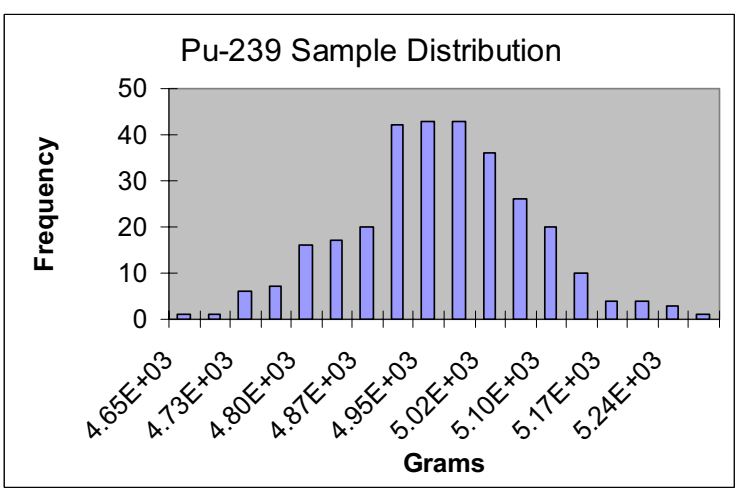

Figure B.2b: Pu-239 Distribution

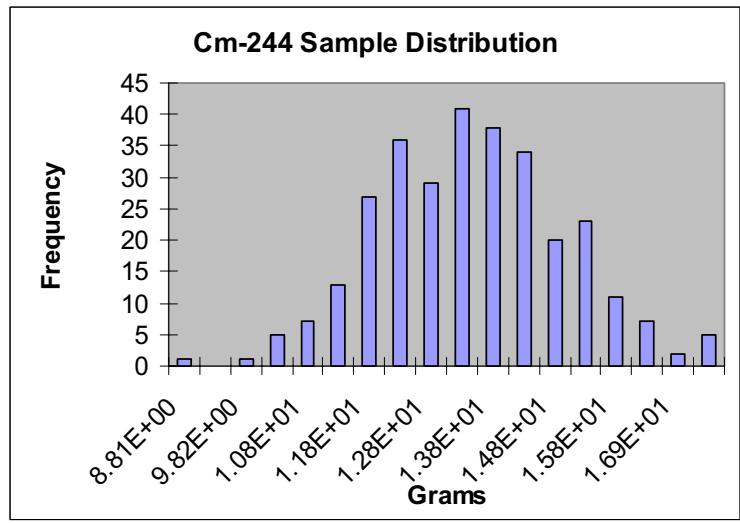

Figure B.2d: Cm-244 Distribution

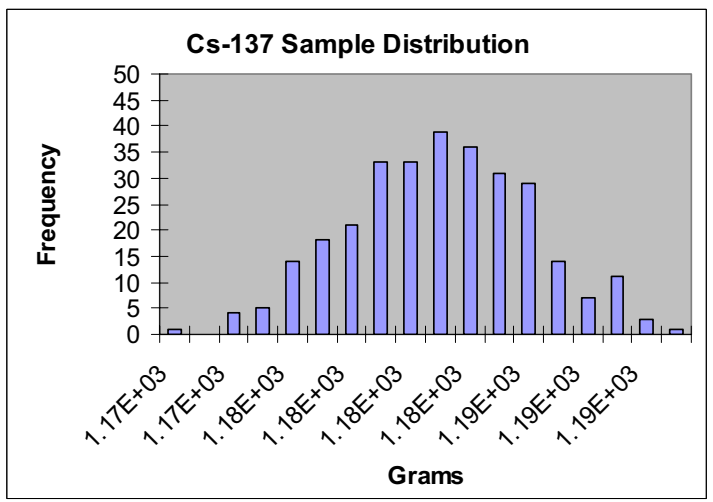

Figure B.2f: Cs-137 Distribution 


\section{Appendix C: Results Tables for Fuel Models}

The following are the generalized results tables for each of the fuel models discussed in the Numerical Results section. The listing follows the order of models presented in the main body of the thesis. Since the nuclides between $\mathrm{Pb}-210$ and $\mathrm{Pa}-231$ were shown to contribute a negligible amount to any of the metrics, they were not included in the isotopics uncertainties lists. Tables are sub-labeled and include the absolute quantity and relative uncertainty to $95 \%$ confidence interval (1.96 standard deviations) for heat load, radioactivity, and radiotoxicity at various time steps, isotopic relative standard deviations for transuranics and fission products, main contributors to uncertainty heat load and radioactivity, and the 5 cross sections causing the most uncertainty in the model (simplified models only). The tables are somewhat different in format between the simplified ORIGEN models and the TRITON models but still follow the same principle. The table for the REBUS model follows the same format as the TRITON tables except "Charge" refers to BOC core loading and "discharge" refers to EOC core loading, both normalized to 1 MTHM. 


\begin{tabular}{|c|c|c|c|c|c|c|}
\hline \multicolumn{7}{|c|}{ Results Table for PWR, 4.5 w/o UOX burned 40 GWD/MTU } \\
\hline \multicolumn{5}{|c|}{ Uncertainty for Key Metrics } & \multirow{2}{*}{\multicolumn{2}{|c|}{$\begin{array}{c}\text { Isotopic Mass } \\
\text { Uncertainties } \\
\text { Actindes (+/- \%) } \\
\end{array}$}} \\
\hline & \multicolumn{2}{|c|}{ Heat } & \multicolumn{2}{|c|}{ Activity } & & \\
\hline & $\mathbf{W}$ & $+/-\%$ & $\mathbf{C i}$ & $+/-\%$ & u235 & 1.338 \\
\hline Charge & $5.817 \mathrm{E}-02$ & N/A & $2.063 \mathrm{E}+00$ & $\mathrm{~N} / \mathrm{A}$ & u236 & 0.702 \\
\hline Discharge & $2.443 \mathrm{E}+06$ & $\mathrm{~N} / \mathrm{A}$ & $2.325 \mathrm{E}+08$ & $\mathrm{~N} / \mathrm{A}$ & u237 & 2.551 \\
\hline $1 \mathrm{yr}$ & $1.178 \mathrm{E}+04$ & $\mathrm{~N} / \mathrm{A}$ & $2.893 E+06$ & $\mathrm{~N} / \mathrm{A}$ & u238 & 0.076 \\
\hline $5 \mathrm{yr}$ & $2.215 \mathrm{E}+03$ & 1.152 & $7.205 E+05$ & 1.173 & np237 & 0.616 \\
\hline $10 \mathrm{yr}$ & $1.423 \mathrm{E}+03$ & 1.166 & $4.982 \mathrm{E}+05$ & 1.119 & np239 & 13.870 \\
\hline $50 \mathrm{yr}$ & $6.628 \mathrm{E}+02$ & 3.037 & $1.603 \mathrm{E}+05$ & 4.180 & pu238 & 1.058 \\
\hline $100 \mathrm{yr}$ & $3.567 \mathrm{E}+02$ & 2.286 & $5.063 E+04$ & 0.601 & pu239 & 0.885 \\
\hline $500 \mathrm{yr}$ & $1.126 \mathrm{E}+02$ & 3.844 & $3.508 \mathrm{E}+03$ & 3.752 & pu240 & 2.766 \\
\hline $1000 \mathrm{yr}$ & $6.221 \mathrm{E}+01$ & 3.280 & $1.966 \mathrm{E}+03$ & 3.174 & pu241 & 2.551 \\
\hline $2500 \mathrm{yr}$ & $2.481 \mathrm{E}+01$ & 2.564 & $8.365 E+02$ & 2.540 & pu242 & 2.625 \\
\hline $5000 \mathrm{yr}$ & $1.818 \mathrm{E}+01$ & 2.642 & $6.274 \mathrm{E}+02$ & 2.590 & am241 & 2.535 \\
\hline \multirow[t]{3}{*}{$10000 \mathrm{yr}$} & $1.346 \mathrm{E}+01$ & 2.257 & $4.705 \mathrm{E}+02$ & 2.199 & am242m & 2.229 \\
\hline & \multicolumn{2}{|c|}{ Ihalation Hazard } & \multicolumn{2}{|c|}{ Ingestion Hazard } & am243 & 13.870 \\
\hline & $\mathrm{m}^{3}$ air & $+/-\%$ & $\mathrm{~m}^{3}$ water & $+/-\%$ & cm242 & 2.229 \\
\hline Charge & $1.133 \mathrm{E}+13$ & $\mathrm{~N} / \mathrm{A}$ & $5.380 \mathrm{E}+06$ & $\mathrm{~N} / \mathrm{A}$ & cm244 & 11.670 \\
\hline Discharge & $9.128 \mathrm{E}+17$ & $\mathrm{~N} / \mathrm{A}$ & $6.723 E+12$ & $\mathrm{~N} / \mathrm{A}$ & cm245 & 10.600 \\
\hline $1 \mathrm{yr}$ & $6.752 \mathrm{E}+17$ & $\mathrm{~N} / \mathrm{A}$ & $7.972 \mathrm{E}+11$ & $\mathrm{~N} / \mathrm{A}$ & \multicolumn{2}{|c|}{ FP (+l- \%) } \\
\hline $5 \mathrm{yr}$ & $6.074 \mathrm{E}+17$ & 3.295 & $3.862 \mathrm{E}+11$ & 1.088 & c 14 & 0.490 \\
\hline $10 \mathrm{yr}$ & $5.968 \mathrm{E}+17$ & 2.913 & $3.171 \mathrm{E}+11$ & 1.092 & se 79 & 0.382 \\
\hline $50 \mathrm{yr}$ & $5.184 \mathrm{E}+17$ & 3.847 & $1.728 \mathrm{E}+11$ & 2.339 & sr 90 & 0.385 \\
\hline $100 \mathrm{yr}$ & $4.405 \mathrm{E}+17$ & 3.196 & $1.055 \mathrm{E}+11$ & 2.512 & tc 99 & 1.880 \\
\hline $500 \mathrm{yr}$ & $2.080 \mathrm{E}+17$ & 3.621 & $3.913 E+10$ & 3.621 & i129 & 0.449 \\
\hline $1000 \mathrm{yr}$ & $1.203 \mathrm{E}+17$ & 3.049 & $2.262 \mathrm{E}+10$ & 3.049 & cs137 & 0.399 \\
\hline $2500 \mathrm{yr}$ & $5.455 \mathrm{E}+16$ & 2.610 & $1.026 \mathrm{E}+10$ & 2.608 & ba137m & 0.399 \\
\hline $5000 \mathrm{yr}$ & $4.133 \mathrm{E}+16$ & 2.642 & $7.778 \mathrm{E}+09$ & 2.639 & y90 & 0.386 \\
\hline $10000 \mathrm{yr}$ & $3.053 \mathrm{E}+16$ & 2.257 & $5.761 \mathrm{E}+09$ & 2.255 & cs134 & 1.209 \\
\hline \multicolumn{5}{|c|}{$\begin{array}{l}\text { Main Contributors to Uncertainty in } \\
\text { Decay Heat and Radioactivity }\end{array}$} & \multicolumn{2}{|c|}{$\begin{array}{c}\text { 1-group Cross Sections } \\
\text { Causing Uncertainty }\end{array}$} \\
\hline & \multicolumn{2}{|c|}{ Heat } & \multicolumn{2}{|c|}{ Activity } & Nuclide & Reaction \\
\hline $5 \mathrm{yr}$ & \multicolumn{2}{|c|}{$\mathrm{Cm}-244$} & \multicolumn{2}{|c|}{ Pu241 } & Am-243 & $(\mathrm{n}, \gamma)$ \\
\hline $10 \mathrm{yr}$ & \multicolumn{2}{|c|}{$\mathrm{Cm}-244, \mathrm{Cs}-134$} & \multicolumn{2}{|c|}{ Pu-241 } & $\mathrm{Pu}-240$ & $(\mathrm{n}, \gamma)$ \\
\hline $50 \mathrm{yr}$ & \multicolumn{2}{|c|}{$\mathrm{Cm}-244, \mathrm{Cs}-134$} & \multicolumn{2}{|c|}{ Pu-241 } & U-235 & (fission) \\
\hline $100 \mathrm{yr}$ & \multicolumn{2}{|c|}{ Am-241 } & \multicolumn{2}{|c|}{$\mathrm{Am}-241$} & Pu-239 & (fission) \\
\hline $500 \mathrm{yr}$ & \multicolumn{2}{|c|}{$\mathrm{Am}-241$} & \multicolumn{2}{|c|}{ Am-241 } & U-234 & $(\mathrm{n}, \gamma)$ \\
\hline $1000 \mathrm{yr}$ & \multicolumn{2}{|c|}{ Am-241 } & \multicolumn{2}{|c|}{ Am-241 } & & \\
\hline $2500 \mathrm{yr}$ & $\mathrm{Pu}-24$ & & $\mathrm{Pu}-24$ & & & \\
\hline $5000 \mathrm{yr}$ & $\mathrm{Pu}-240 \mathrm{Pu}$ & -239 & Pu-240, P & -239 & & \\
\hline $10000 \mathrm{yr}$ & $\mathrm{Pu}-240 \mathrm{Pu}$ & -239 & $\mathrm{Pu}-240, \mathrm{P}$ & -239 & & \\
\hline
\end{tabular}

Table C.1: Results table for the PWR model using ESM for uncertainty. 


\begin{tabular}{|c|c|c|c|c|c|c|}
\hline \multicolumn{7}{|c|}{ Results Table for PWR, 4.5 w/o UOX burned 40 GWD/MTU } \\
\hline \multicolumn{5}{|c|}{ Uncertainty for Key Metrics } & \multirow{2}{*}{\multicolumn{2}{|c|}{\begin{tabular}{|l} 
Isotopic Mass \\
Uncertainties \\
Actindes (+/- \%) \\
\end{tabular}}} \\
\hline & \multicolumn{2}{|c|}{ Heat } & \multicolumn{2}{|c|}{ Activity } & & \\
\hline & $\mathbf{W}$ & $+/-\%$ & $\mathbf{C i}$ & $+/-\%$ & u235 & 1.312 \\
\hline Charge & 5.817E-02 & $\mathrm{N} / \mathrm{A}$ & $2.063 E+00$ & $\mathrm{~N} / \mathrm{A}$ & u236 & 0.712 \\
\hline Discharge & $2.443 E+06$ & $\mathrm{~N} / \mathrm{A}$ & $2.325 \mathrm{E}+08$ & $\mathrm{~N} / \mathrm{A}$ & u237 & 2.521 \\
\hline $1 \mathrm{yr}$ & $1.178 \mathrm{E}+04$ & $\mathrm{~N} / \mathrm{A}$ & $2.893 E+06$ & $\mathrm{~N} / \mathrm{A}$ & u238 & 0.078 \\
\hline $5 \mathrm{yr}$ & $2.215 E+03$ & 1.113 & $7.205 E+05$ & 1.156 & np237 & 0.605 \\
\hline $10 \mathrm{yr}$ & $1.423 E+03$ & 1.133 & $4.982 \mathrm{E}+05$ & 1.103 & np239 & 13.643 \\
\hline $50 \mathrm{yr}$ & $6.628 E+02$ & 2.935 & $1.603 \mathrm{E}+05$ & 4.122 & pu238 & 1.050 \\
\hline $100 \mathrm{yr}$ & $3.567 \mathrm{E}+02$ & 2.255 & $5.063 E+04$ & 0.588 & pu239 & 0.806 \\
\hline $500 \mathrm{yr}$ & $1.126 \mathrm{E}+02$ & 3.788 & $3.508 \mathrm{E}+03$ & 3.696 & pu240 & 2.566 \\
\hline $1000 \mathrm{yr}$ & $6.221 \mathrm{E}+01$ & 3.215 & $1.966 \mathrm{E}+03$ & 3.109 & pu241 & 2.521 \\
\hline $2500 \mathrm{yr}$ & $2.481 \mathrm{E}+01$ & 2.396 & $8.365 E+02$ & 2.379 & pu242 & 2.598 \\
\hline $5000 \mathrm{yr}$ & $1.818 \mathrm{E}+01$ & 2.457 & $6.274 \mathrm{E}+02$ & 2.417 & am241 & 2.502 \\
\hline \multirow[t]{3}{*}{$10000 \mathrm{yr}$} & $1.346 \mathrm{E}+01$ & 2.094 & $4.705 \mathrm{E}+02$ & 2.049 & am242m & 2.172 \\
\hline & \multicolumn{2}{|c|}{ Inhalation Hazard } & \multicolumn{2}{|c|}{ Ingestion Hazard } & am243 & 13.643 \\
\hline & $\mathrm{m}^{3}$ air & $+1-\%$ & $\mathrm{~m}^{3}$ water & $+/-\%$ & cm242 & 2.172 \\
\hline Charge & $1.133 E+13$ & $\mathrm{~N} / \mathrm{A}$ & $5.380 \mathrm{E}+06$ & $\mathrm{~N} / \mathrm{A}$ & cm244 & 11.340 \\
\hline Discharge & $9.128 \mathrm{E}+17$ & $\mathrm{~N} / \mathrm{A}$ & $6.723 \mathrm{E}+12$ & $\mathrm{~N} / \mathrm{A}$ & cm245 & 10.251 \\
\hline $1 \mathrm{yr}$ & $6.752 \mathrm{E}+17$ & $\mathrm{~N} / \mathrm{A}$ & $7.972 E+11$ & $\mathrm{~N} / \mathrm{A}$ & \multicolumn{2}{|c|}{ FP (+/- \%) } \\
\hline $5 \mathrm{yr}$ & $6.074 \mathrm{E}+17$ & 3.216 & $3.862 \mathrm{E}+11$ & 1.059 & c 14 & 0.452 \\
\hline $10 \mathrm{yr}$ & $5.968 \mathrm{E}+17$ & 2.846 & $3.171 \mathrm{E}+11$ & 1.066 & se 79 & 0.366 \\
\hline $50 \mathrm{yr}$ & $5.184 \mathrm{E}+17$ & 3.755 & $1.728 \mathrm{E}+11$ & 2.278 & sr 90 & 0.375 \\
\hline $100 \mathrm{yr}$ & $4.405 \mathrm{E}+17$ & 3.152 & $1.055 \mathrm{E}+11$ & 2.477 & tc 99 & 1.970 \\
\hline $500 \mathrm{yr}$ & $2.080 \mathrm{E}+17$ & 3.564 & $3.913 E+10$ & 3.564 & $\mathbf{i 1 2 9}$ & 0.419 \\
\hline $1000 \mathrm{yr}$ & $1.203 \mathrm{E}+17$ & 2.976 & $2.262 \mathrm{E}+10$ & 2.976 & $\operatorname{cs137}$ & 0.382 \\
\hline $2500 \mathrm{yr}$ & $5.455 \mathrm{E}+16$ & 2.430 & $1.026 \mathrm{E}+10$ & 2.428 & ba137m & 0.382 \\
\hline $5000 \mathrm{yr}$ & $4.133 E+16$ & 2.452 & $7.778 \mathrm{E}+09$ & 2.450 & y90 & 0.375 \\
\hline $10000 \mathrm{yr}$ & $3.053 \mathrm{E}+16$ & 2.091 & $5.761 \mathrm{E}+09$ & 2.089 & cs134 & 1.138 \\
\hline \multicolumn{5}{|c|}{$\begin{array}{l}\text { Main Contributors to Uncertainty in } \\
\text { Decay Heat and Radioactivity }\end{array}$} & \multicolumn{2}{|c|}{$\begin{array}{c}\text { 1-group Cross Sections } \\
\text { Causing Uncertainty } \\
\end{array}$} \\
\hline & \multicolumn{2}{|c|}{ Heat } & \multicolumn{2}{|c|}{ Activity } & Nuclide & Reaction \\
\hline $5 \mathrm{yr}$ & \multicolumn{2}{|c|}{$\mathrm{Cm}-244$} & \multicolumn{2}{|c|}{ Pu241 } & Am-243 & $(\mathrm{n}, \gamma)$ \\
\hline $10 \mathrm{yr}$ & \multicolumn{2}{|c|}{$\mathrm{Cm}-244, \mathrm{Cs}-134$} & \multicolumn{2}{|c|}{$\mathrm{Pu}-241$} & $\mathrm{Pu}-240$ & $(\mathrm{n}, \gamma)$ \\
\hline $50 \mathrm{yr}$ & \multicolumn{2}{|c|}{$\mathrm{Cm}-244, \mathrm{Cs}-134$} & \multicolumn{2}{|c|}{ Pu-241 } & $\mathrm{U}-235$ & (fission) \\
\hline $100 \mathrm{yr}$ & \multicolumn{2}{|c|}{$\mathrm{Am}-241$} & \multicolumn{2}{|c|}{$\mathrm{Am}-241$} & Pu-239 & (fission) \\
\hline $500 \mathrm{yr}$ & \multicolumn{2}{|c|}{ Am-241 } & \multicolumn{2}{|c|}{$\mathrm{Am}-241$} & $\mathrm{U}-234$ & $(\mathrm{n}, \gamma)$ \\
\hline $1000 \mathrm{yr}$ & \multicolumn{2}{|c|}{$\mathrm{Am}-241$} & \multicolumn{2}{|c|}{ Am-241 } & & \\
\hline $2500 \mathrm{yr}$ & Pu-24 & & $\mathrm{Pu}-24$ & & & \\
\hline $5000 \mathrm{yr}$ & $\mathrm{Pu}-240 \mathrm{Pl}$ & -239 & $\mathrm{Pu}-240, \mathrm{Pu}$ & -239 & & \\
\hline $10000 \mathrm{yr}$ & $\mathrm{Pu}-240 \mathrm{Pu}$ & -239 & $\mathrm{Pu}-240, \mathrm{Pu}$ & -239 & & \\
\hline
\end{tabular}

Table C.2: Results table for PWR fuel in the simplified model. 


\begin{tabular}{|c|c|c|c|c|c|c|}
\hline \multicolumn{7}{|c|}{ Results Table for LWR, 4.5 w/o l } \\
\hline \multicolumn{5}{|c|}{ Uncertainty for Key Metrics } & \multirow{2}{*}{\multicolumn{2}{|c|}{$\begin{array}{c}\begin{array}{l}\text { Isotopic Mass } \\
\text { Uncertainties }\end{array} \\
\text { Actindes (+/- \%) }\end{array}$}} \\
\hline & \multicolumn{2}{|c|}{ Heat } & \multicolumn{2}{|c|}{ Activity } & & \\
\hline & $\mathbf{W}$ & $+/-\%$ & $\mathbf{C i}$ & $+/-\%$ & u235 & 6.184 \\
\hline Charge & $5.817 \mathrm{E}-02$ & $\mathrm{~N} / \mathrm{A}$ & $2.063 \mathrm{E}+00$ & $\mathrm{~N} / \mathrm{A}$ & u236 & 1.364 \\
\hline Discharge & $5.906 \mathrm{E}+04$ & $\mathrm{~N} / \mathrm{A}$ & $2.255 \mathrm{E}+07$ & $\mathrm{~N} / \mathrm{A}$ & u237 & 3.708 \\
\hline $1 \mathrm{yr}$ & $3.121 \mathrm{E}+03$ & $\mathrm{~N} / \mathrm{A}$ & $7.167 \mathrm{E}+05$ & $\mathrm{~N} / \mathrm{A}$ & u238 & 0.089 \\
\hline 5 yr & $1.711 \mathrm{E}+03$ & 1.148 & $5.456 \mathrm{E}+05$ & 1.426 & np237 & 1.032 \\
\hline $10 \mathrm{yr}$ & $1.292 \mathrm{E}+03$ & 0.966 & $4.494 \mathrm{E}+05$ & 1.353 & np239 & 12.698 \\
\hline $50 \mathrm{yr}$ & $6.104 \mathrm{E}+02$ & 1.649 & $1.562 \mathrm{E}+05$ & 0.719 & pu238 & 2.467 \\
\hline $100 \mathrm{yr}$ & $3.120 \mathrm{E}+02$ & 3.037 & $4.902 \mathrm{E}+04$ & 0.730 & pu239 & 2.202 \\
\hline $500 \mathrm{yr}$ & $9.464 \mathrm{E}+01$ & 5.269 & $2.925 \mathrm{E}+03$ & 5.124 & pu240 & 2.837 \\
\hline $1000 \mathrm{yr}$ & $5.463 \mathrm{E}+01$ & 4.340 & $1.720 \mathrm{E}+03$ & 4.181 & pu241 & 3.708 \\
\hline $2500 \mathrm{yr}$ & $2.434 \mathrm{E}+01$ & 3.304 & $8.046 \mathrm{E}+02$ & 3.222 & pu242 & 3.995 \\
\hline 5000 yr & $1.815 \mathrm{E}+01$ & 3.485 & $6.092 \mathrm{E}+02$ & 3.362 & am241 & 3.675 \\
\hline \multirow[t]{3}{*}{$10000 \mathrm{yr}$} & $1.309 \mathrm{E}+01$ & 3.348 & $4.433 E+02$ & 3.200 & am242m & 3.169 \\
\hline & \multicolumn{2}{|c|}{ Inhalation Hazard } & \multicolumn{2}{|c|}{ Ingestion Hazard } & am243 & 12.698 \\
\hline & $\mathrm{m}^{3}$ air & $+/-\%$ & $\mathrm{~m}^{3}$ water & $+/-\%$ & cm242 & 3.169 \\
\hline Charge & $1.133 \mathrm{E}+13$ & $\mathrm{~N} / \mathrm{A}$ & $5.380 \mathrm{E}+06$ & $\mathrm{~N} / \mathrm{A}$ & cm244 & 11.464 \\
\hline Discharge & $5.939 \mathrm{E}+17$ & $\mathrm{~N} / \mathrm{A}$ & $1.504 \mathrm{E}+12$ & $\mathrm{~N} / \mathrm{A}$ & cm245 & 11.231 \\
\hline $1 \mathrm{yr}$ & $4.983 E+17$ & $\mathrm{~N} / \mathrm{A}$ & $4.863 E+11$ & $\mathrm{~N} / \mathrm{A}$ & \multicolumn{2}{|c|}{ FP (+/- \%) } \\
\hline $5 \mathrm{yr}$ & $4.729 \mathrm{E}+17$ & 3.570 & $3.530 \mathrm{E}+11$ & 1.135 & c 14 & 0.683 \\
\hline $10 \mathrm{yr}$ & $4.671 \mathrm{E}+17$ & 3.337 & $2.960 \mathrm{E}+11$ & 1.158 & se 79 & 0.415 \\
\hline $50 \mathrm{yr}$ & $4.163 \mathrm{E}+17$ & 4.057 & $1.551 \mathrm{E}+11$ & 2.092 & sr 90 & 0.713 \\
\hline $100 \mathrm{yr}$ & $3.581 \mathrm{E}+17$ & 4.589 & $9.047 E+10$ & 3.424 & tc 99 & 1.644 \\
\hline $500 \mathrm{yr}$ & $1.776 \mathrm{E}+17$ & 4.902 & $3.341 \mathrm{E}+10$ & 4.902 & i129 & 0.569 \\
\hline $1000 \mathrm{yr}$ & $1.078 \mathrm{E}+17$ & 3.994 & $2.026 \mathrm{E}+10$ & 3.995 & cs137 & 0.335 \\
\hline $2500 \mathrm{yr}$ & $5.414 \mathrm{E}+16$ & 3.376 & $1.018 \mathrm{E}+10$ & 3.373 & ba137m & 0.335 \\
\hline 5000 yr & $4.149 \mathrm{E}+16$ & 3.514 & $7.799 \mathrm{E}+09$ & 3.511 & y90 & 0.713 \\
\hline $10000 \mathrm{yr}$ & $2.991 \mathrm{E}+16$ & 3.381 & $5.623 \mathrm{E}+09$ & 3.378 & $\operatorname{cs} 134$ & 1.956 \\
\hline \multicolumn{5}{|c|}{$\begin{array}{l}\text { Main Contributors to Uncertainty in } \\
\text { Decay Heat and Radioactivity }\end{array}$} & \multicolumn{2}{|c|}{$\begin{array}{l}\text { 1-group Cross Section } \\
\text { Causing Uncertainty }\end{array}$} \\
\hline & \multicolumn{2}{|c|}{ Heat } & \multicolumn{2}{|c|}{ Activity } & Nuclide & Reaction \\
\hline $5 \mathrm{yr}$ & \multicolumn{2}{|c|}{$\mathrm{Cm}-244, \mathrm{Cs}-134$} & \multicolumn{2}{|c|}{ Pu241 } & Am-243 & $(n, y)$ \\
\hline $10 \mathrm{yr}$ & \multicolumn{2}{|c|}{$\mathrm{Cm}-244, \mathrm{Y}-90$} & \multicolumn{2}{|c|}{ Pu-241 } & Pu-240 & $(n, y)$ \\
\hline $50 \mathrm{yr}$ & \multicolumn{2}{|c|}{ Am-241 } & \multicolumn{2}{|c|}{ Pu-241 } & U-235 & (fission) \\
\hline $100 \mathrm{yr}$ & \multicolumn{2}{|c|}{ Am-241 } & \multicolumn{2}{|c|}{ Am-241 } & Pu-239 & (fission) \\
\hline $500 \mathrm{yr}$ & \multicolumn{2}{|c|}{ Am-241 } & \multicolumn{2}{|c|}{ Am-241 } & U-234 & $(n, y)$ \\
\hline $1000 \mathrm{yr}$ & \multicolumn{2}{|c|}{ Am-241 } & \multicolumn{2}{|c|}{ Am-241 } & & \\
\hline $2500 \mathrm{yr}$ & $\mathrm{Pu}-240 \mathrm{Pu}$ & 239 & $\mathrm{Pu}-240, \mathrm{Pu}$ & -239 & & \\
\hline $5000 \mathrm{yr}$ & $\mathrm{Pu}-240 \mathrm{Pl}$ & 239 & $\mathrm{Pu}-240, \mathrm{Pu}$ & -239 & & \\
\hline $10000 \mathrm{yr}$ & $\mathrm{Pu}-240 \mathrm{Pl}$ & 239 & $\mathrm{Pu}-240, \mathrm{Pu}$ & -239 & & \\
\hline
\end{tabular}

Table C.3: Results table for typical LWR fuel simplified model. 


\begin{tabular}{|c|c|c|c|c|c|c|}
\hline \multicolumn{7}{|c|}{$\begin{array}{c}\text { Results Table for BWR, 0\% Void, } 4.5 \text { w/o UOX } \\
\text { burned } 40 \text { GWD/MTU }\end{array}$} \\
\hline \multicolumn{5}{|c|}{ Uncertainty for Key Metrics } & \multirow{2}{*}{\multicolumn{2}{|c|}{$\begin{array}{c}\begin{array}{c}\text { Isotopic Mass } \\
\text { Uncertainties }\end{array} \\
\text { Actindes (+/- \%) } \\
\end{array}$}} \\
\hline & \multicolumn{2}{|c|}{ Heat } & \multicolumn{2}{|c|}{ Activity } & & \\
\hline & $\mathbf{w}$ & $+/-\%$ & $\mathbf{C i}$ & $+/-\%$ & u235 & 1.360 \\
\hline Charge & $2.063 \mathrm{E}+00$ & $\mathrm{~N} / \mathrm{A}$ & $5.817 \mathrm{E}-02$ & $\mathrm{~N} / \mathrm{A}$ & u236 & 0.481 \\
\hline Discharge & $2.229 \mathrm{E}+08$ & $\mathrm{~N} / \mathrm{A}$ & $2.368 \mathrm{E}+06$ & $\mathrm{~N} / \mathrm{A}$ & u237 & 2.105 \\
\hline $1 \mathrm{yr}$ & $2.767 \mathrm{E}+06$ & $\mathrm{~N} / \mathrm{A}$ & $1.095 \mathrm{E}+04$ & $\mathrm{~N} / \mathrm{A}$ & u238 & 0.055 \\
\hline $5 \mathrm{yr}$ & $6.661 \mathrm{E}+05$ & 0.491 & $2.014 \mathrm{E}+03$ & 0.617 & np237 & 0.541 \\
\hline $10 \mathrm{yr}$ & $4.602 \mathrm{E}+05$ & 0.426 & $1.304 \mathrm{E}+03$ & 0.585 & np239 & 8.910 \\
\hline $50 \mathrm{yr}$ & $1.546 \mathrm{E}+05$ & 0.719 & $5.510 \mathrm{E}+02$ & 0.368 & pu238 & 1.102 \\
\hline $100 \mathrm{yr}$ & $4.824 \mathrm{E}+04$ & 1.430 & $2.536 \mathrm{E}+02$ & 0.373 & pu239 & 0.817 \\
\hline $500 \mathrm{yr}$ & $2.098 \mathrm{E}+03$ & 2.936 & $6.619 \mathrm{E}+01$ & 2.851 & pu240 & 2.157 \\
\hline $1000 \mathrm{yr}$ & $1.256 \mathrm{E}+03$ & 2.505 & $3.920 \mathrm{E}+01$ & 2.422 & pu241 & 2.105 \\
\hline $2500 \mathrm{yr}$ & $6.293 \mathrm{E}+02$ & 2.411 & $1.850 \mathrm{E}+01$ & 2.336 & pu242 & 2.011 \\
\hline $5000 \mathrm{yr}$ & $4.829 \mathrm{E}+02$ & 2.463 & $1.388 \mathrm{E}+01$ & 2.354 & am241 & 2.089 \\
\hline \multirow[t]{3}{*}{$10000 \mathrm{yr}$} & $3.510 \mathrm{E}+02$ & 2.135 & $9.855 \mathrm{E}+00$ & 2.017 & am242m & 1.807 \\
\hline & \multicolumn{2}{|c|}{ Inhalation Hazard } & \multicolumn{2}{|c|}{ Ingestion Hazard } & am243 & 8.910 \\
\hline & $\mathrm{m}^{3}$ air & $+/-\%$ & $\mathrm{~m}^{3}$ water & $+/-\%$ & $\mathrm{~cm} 242$ & 1.806 \\
\hline Charge & $1.133 E+13$ & $\mathrm{~N} / \mathrm{A}$ & $5.380 \mathrm{E}+06$ & $\mathrm{~N} / \mathrm{A}$ & cm244 & 7.416 \\
\hline Discharge & $5.477 \mathrm{E}+17$ & $\mathrm{~N} / \mathrm{A}$ & $6.431 \mathrm{E}+12$ & $\mathrm{~N} / \mathrm{A}$ & cm245 & 6.816 \\
\hline $1 \mathrm{yr}$ & $3.662 \mathrm{E}+17$ & $\mathrm{~N} / \mathrm{A}$ & $7.115 \mathrm{E}+11$ & $\mathrm{~N} / \mathrm{A}$ & \multicolumn{2}{|c|}{ FP (+/- \%) } \\
\hline $5 \mathrm{yr}$ & $3.192 \mathrm{E}+17$ & 1.854 & $3.352 \mathrm{E}+11$ & 0.500 & c 14 & 0.392 \\
\hline $10 \mathrm{yr}$ & $3.155 \mathrm{E}+17$ & 1.740 & $2.731 \mathrm{E}+11$ & 0.510 & se 79 & 0.316 \\
\hline $50 \mathrm{yr}$ & $2.838 \mathrm{E}+17$ & 2.243 & $1.326 \mathrm{E}+11$ & 0.941 & sr 90 & 0.322 \\
\hline $100 \mathrm{yr}$ & $2.455 \mathrm{E}+17$ & 2.555 & $6.998 \mathrm{E}+10$ & 1.693 & tc 99 & 1.309 \\
\hline $500 \mathrm{yr}$ & $1.252 \mathrm{E}+17$ & 2.752 & $2.355 \mathrm{E}+10$ & 2.752 & i129 & 0.362 \\
\hline $1000 \mathrm{yr}$ & $7.806 \mathrm{E}+16$ & 2.387 & $1.468 \mathrm{E}+10$ & 2.386 & cs137 & 0.326 \\
\hline $2500 \mathrm{yr}$ & $4.122 \mathrm{E}+16$ & 2.474 & $7.753 \mathrm{E}+09$ & 2.472 & ba137m & 0.326 \\
\hline 5000 yr & $3.164 \mathrm{E}+16$ & 2.484 & $5.956 \mathrm{E}+09$ & 2.482 & y90 & 0.322 \\
\hline $10000 \mathrm{yr}$ & $2.239 \mathrm{E}+16$ & 2.157 & $4.226 \mathrm{E}+09$ & 2.155 & cs134 & 0.962 \\
\hline \multicolumn{5}{|c|}{$\begin{array}{l}\text { Main Contributors to Uncertainty in } \\
\text { Decay Heat and Radioactivity }\end{array}$} & \multicolumn{2}{|c|}{$\begin{array}{c}\text { 1-group Cross Section } \\
\text { Causing Uncertainty }\end{array}$} \\
\hline & \multicolumn{2}{|c|}{ Heat } & \multicolumn{2}{|c|}{ Activity } & Nuclide & Reaction \\
\hline $5 \mathrm{yr}$ & \multicolumn{2}{|c|}{$\mathrm{Cm}-244, \mathrm{Cs}-134$} & \multicolumn{2}{|c|}{ Pu241 } & Am-243 & $(\mathrm{n}, \gamma)$ \\
\hline $10 \mathrm{yr}$ & \multicolumn{2}{|c|}{$\begin{array}{c}\text { Cm-244,Y-90, Ba- } \\
137 m\end{array}$} & \multicolumn{2}{|c|}{ Pu-241 } & Pu-240 & $(\mathrm{n}, \gamma)$ \\
\hline $50 \mathrm{yr}$ & \multicolumn{2}{|c|}{$\mathrm{Am}-241$} & \multicolumn{2}{|c|}{ Pu-241 } & U-235 & (fission) \\
\hline $100 \mathrm{yr}$ & \multicolumn{2}{|c|}{ Am-241 } & \multicolumn{2}{|c|}{$\mathrm{Pu}-241$} & Pu-239 & (fission) \\
\hline $500 \mathrm{yr}$ & \multicolumn{2}{|c|}{ Am-241 } & \multicolumn{2}{|c|}{ Am-241 } & $\mathrm{U}-234$ & $(\mathrm{n}, \gamma)$ \\
\hline $1000 \mathrm{yr}$ & \multicolumn{2}{|c|}{ Am-241 } & \multicolumn{2}{|c|}{ Am-241 } & & \\
\hline $2500 \mathrm{yr}$ & $\mathrm{Pu}-24$ & & $\mathrm{Pu}-24$ & & & \\
\hline $5000 \mathrm{yr}$ & Pu-240 P & 239 & $\mathrm{Pu}-240, \mathrm{P}$ & -239 & & \\
\hline $10000 \mathrm{yr}$ & Pu-240 P & 239 & Pu-240, P & -239 & & \\
\hline
\end{tabular}

Table C.4: Results table for BWR fuel burned at $0 \%$ void. 


\begin{tabular}{|c|c|c|c|c|c|c|}
\hline \multicolumn{7}{|c|}{$\begin{array}{r}\text { Results Table for BWR, } \\
\text { burned } 40\end{array}$} \\
\hline \multicolumn{5}{|c|}{ Uncertainty for Key Metrics } & \multirow{2}{*}{\multicolumn{2}{|c|}{$\begin{array}{c}\text { Isotopic Mass } \\
\text { Uncertainties } \\
\text { Actindes (+/- \%) }\end{array}$}} \\
\hline & \multicolumn{2}{|c|}{ Heat } & \multicolumn{2}{|c|}{ Activity } & & \\
\hline & w & $+/-\%$ & $\mathbf{C i}$ & $+/-\%$ & u235 & 1.472 \\
\hline Charge & $2.063 \mathrm{E}+00$ & $\mathrm{~N} / \mathrm{A}$ & $5.817 \mathrm{E}-02$ & $\mathrm{~N} / \mathrm{A}$ & u236 & 0.608 \\
\hline Discharge & $2.274 \mathrm{E}+08$ & $\mathrm{~N} / \mathrm{A}$ & $2.405 \mathrm{E}+06$ & $\mathrm{~N} / \mathrm{A}$ & u237 & 2.390 \\
\hline $1 \mathrm{yr}$ & $2.817 \mathrm{E}+06$ & $\mathrm{~N} / \mathrm{A}$ & $1.128 \mathrm{E}+04$ & $\mathrm{~N} / \mathrm{A}$ & u238 & 0.065 \\
\hline $5 \mathrm{yr}$ & $6.855 \mathrm{E}+05$ & 0.674 & $2.087 \mathrm{E}+03$ & 0.854 & np237 & 0.593 \\
\hline $10 \mathrm{yr}$ & $4.735 \mathrm{E}+05$ & 0.631 & $1.344 \mathrm{E}+03$ & 0.811 & np239 & 11.086 \\
\hline $50 \mathrm{yr}$ & $1.565 \mathrm{E}+05$ & 0.982 & $5.912 E+02$ & 0.468 & pu238 & 1.101 \\
\hline $100 \mathrm{yr}$ & $4.907 \mathrm{E}+04$ & 1.853 & $2.916 \mathrm{E}+02$ & 0.475 & pu239 & 0.867 \\
\hline $500 \mathrm{yr}$ & $2.632 \mathrm{E}+03$ & 3.436 & $8.375 E+01$ & 3.343 & pu240 & 2.381 \\
\hline $1000 \mathrm{yr}$ & $1.533 \mathrm{E}+03$ & 2.897 & $4.816 \mathrm{E}+01$ & 2.798 & pu241 & 2.390 \\
\hline $2500 \mathrm{yr}$ & $7.191 \mathrm{E}+02$ & 2.478 & $2.125 \mathrm{E}+01$ & 2.418 & pu242 & 2.252 \\
\hline $5000 \mathrm{yr}$ & $5.470 \mathrm{E}+02$ & 2.539 & $1.581 \mathrm{E}+01$ & 2.447 & am241 & 2.372 \\
\hline \multirow[t]{3}{*}{$10000 \mathrm{yr}$} & $4.023 E+02$ & 2.190 & $1.142 \mathrm{E}+01$ & 2.093 & am242m & 2.071 \\
\hline & \multicolumn{2}{|c|}{ Inhalation Hazard } & \multicolumn{2}{|c|}{ Ingestion Hazard } & am243 & 11.086 \\
\hline & $\mathrm{m}^{3}$ air & $+/-\%$ & $\mathrm{~m}^{3}$ water & $+/-\%$ & cm242 & 2.070 \\
\hline Charge & $1.133 \mathrm{E}+13$ & $\mathrm{~N} / \mathrm{A}$ & $5.380 \mathrm{E}+06$ & $\mathrm{~N} / \mathrm{A}$ & cm244 & 9.233 \\
\hline Discharge & $6.788 \mathrm{E}+17$ & $\mathrm{~N} / \mathrm{A}$ & $6.560 \mathrm{E}+12$ & $\mathrm{~N} / \mathrm{A}$ & cm245 & 8.431 \\
\hline $1 \mathrm{yr}$ & $4.769 \mathrm{E}+17$ & $\mathrm{~N} / \mathrm{A}$ & $7.444 \mathrm{E}+11$ & $\mathrm{~N} / \mathrm{A}$ & \multicolumn{2}{|c|}{ FP (+/- \%) } \\
\hline $5 \mathrm{yr}$ & $4.228 \mathrm{E}+17$ & 2.369 & $3.535 \mathrm{E}+11$ & 0.683 & c 14 & 0.441 \\
\hline $10 \mathrm{yr}$ & $4.171 \mathrm{E}+17$ & 2.166 & $2.886 \mathrm{E}+11$ & 0.698 & se 79 & 0.363 \\
\hline $50 \mathrm{yr}$ & $3.703 \mathrm{E}+17$ & 2.562 & $1.472 \mathrm{E}+11$ & 1.242 & sr 90 & 0.381 \\
\hline $100 \mathrm{yr}$ & $3.180 \mathrm{E}+17$ & 2.935 & $8.316 \mathrm{E}+10$ & 2.117 & tc 99 & 1.609 \\
\hline $500 \mathrm{yr}$ & $1.569 \mathrm{E}+17$ & 3.217 & $2.951 \mathrm{E}+10$ & 3.217 & i129 & 0.408 \\
\hline $1000 \mathrm{yr}$ & $9.474 \mathrm{E}+16$ & 2.710 & $1.782 \mathrm{E}+10$ & 2.710 & cs137 & 0.374 \\
\hline $2500 \mathrm{yr}$ & $4.713 \mathrm{E}+16$ & 2.538 & $8.863 E+09$ & 2.536 & ba137m & 0.374 \\
\hline $5000 \mathrm{yr}$ & $3.602 \mathrm{E}+16$ & 2.555 & $6.779 \mathrm{E}+09$ & 2.553 & y90 & 0.381 \\
\hline $10000 \mathrm{yr}$ & $2.594 \mathrm{E}+16$ & 2.206 & $4.894 \mathrm{E}+09$ & 2.204 & cs134 & 1.063 \\
\hline \multicolumn{5}{|c|}{$\begin{array}{l}\text { Main Contributors to Uncertainty in } \\
\text { Decay Heat and Radioactivity }\end{array}$} & \multicolumn{2}{|c|}{$\begin{array}{c}\text { 1-group Cross Sections } \\
\text { Causing Uncertainty }\end{array}$} \\
\hline & \multicolumn{2}{|c|}{ Heat } & \multicolumn{2}{|c|}{ Activity } & Nuclide & Reaction \\
\hline $5 \mathrm{yr}$ & \multicolumn{2}{|c|}{$\mathrm{Cm}-244, \mathrm{Cs}-134$} & \multicolumn{2}{|c|}{ Pu241 } & $\mathrm{Am}-243$ & $(\mathrm{n}, \gamma)$ \\
\hline $10 \mathrm{yr}$ & \multicolumn{2}{|c|}{$\begin{array}{l}\mathrm{Cm}-244, Y-90 \\
\mathrm{Ba}-137 \mathrm{~m}\end{array}$} & \multicolumn{2}{|c|}{ Pu-241 } & Pu-240 & $(\mathrm{n}, \gamma)$ \\
\hline $50 \mathrm{yr}$ & \multicolumn{2}{|c|}{ Am-241 } & \multicolumn{2}{|c|}{ Pu-241 } & U-235 & (fission) \\
\hline $100 \mathrm{yr}$ & \multicolumn{2}{|c|}{ Am-241 } & \multicolumn{2}{|c|}{ Am-241 } & Pu-239 & (fission) \\
\hline $500 \mathrm{yr}$ & \multicolumn{2}{|c|}{ Am-241 } & \multicolumn{2}{|c|}{ Am-241 } & $\mathrm{U}-234$ & $(\mathrm{n}, \gamma)$ \\
\hline $1000 \mathrm{yr}$ & \multicolumn{2}{|c|}{ Am-241 } & \multicolumn{2}{|c|}{$\mathrm{Am}-241$} & & \\
\hline $2500 \mathrm{yr}$ & Pu-24 & & $\mathrm{Pu}-24$ & & & \\
\hline $5000 \mathrm{yr}$ & Pu-240 Pu & -239 & Pu-240, P & -239 & & \\
\hline $10000 \mathrm{yr}$ & Pu-240 Pu & -239 & Pu-240, P & -239 & & \\
\hline
\end{tabular}

Table C.5: Results table for BWR fuel burned at 35\% void. 


\begin{tabular}{|c|c|c|c|c|c|c|}
\hline \multicolumn{7}{|c|}{$\begin{array}{c}\text { Results Table for BWR, 50\% Void, } 4.5 \text { w/o UOX } \\
\text { burned } 40 \text { GWD/MTU }\end{array}$} \\
\hline \multicolumn{5}{|c|}{ Uncertainty for Key Metrics } & \multirow{2}{*}{\multicolumn{2}{|c|}{$\begin{array}{c}\begin{array}{c}\text { Isotopic Mass } \\
\text { Uncertainties }\end{array} \\
\text { Actindes (+/- \%) } \\
\end{array}$}} \\
\hline & \multicolumn{2}{|c|}{ Heat } & \multicolumn{2}{|c|}{ Activity } & & \\
\hline & $\mathbf{W}$ & $+/-\%$ & $\mathbf{C i}$ & $+/-\%$ & u235 & 1.757 \\
\hline Charge & $2.063 E+00$ & $\mathrm{~N} / \mathrm{A}$ & $5.817 \mathrm{E}-02$ & $\mathrm{~N} / \mathrm{A}$ & u236 & 0.731 \\
\hline Discharge & $2.303 E+08$ & $\mathrm{~N} / \mathrm{A}$ & $2.425 \mathrm{E}+06$ & $\mathrm{~N} / \mathrm{A}$ & u237 & 2.492 \\
\hline $1 \mathrm{yr}$ & $2.853 E+06$ & $\mathrm{~N} / \mathrm{A}$ & $1.153 E+04$ & $\mathrm{~N} / \mathrm{A}$ & u238 & 0.069 \\
\hline $5 \mathrm{yr}$ & $7.015 \mathrm{E}+05$ & 0.880 & $2.146 \mathrm{E}+03$ & 1.025 & np237 & 0.638 \\
\hline $10 \mathrm{yr}$ & $4.848 \mathrm{E}+05$ & 0.858 & $1.379 \mathrm{E}+03$ & 0.977 & np239 & 12.685 \\
\hline $50 \mathrm{yr}$ & $1.582 \mathrm{E}+05$ & 1.171 & $6.249 \mathrm{E}+02$ & 0.548 & pu238 & 1.147 \\
\hline $100 \mathrm{yr}$ & $4.979 \mathrm{E}+04$ & 2.098 & $3.230 \mathrm{E}+02$ & 0.556 & pu239 & 0.874 \\
\hline $500 \mathrm{yr}$ & $3.074 \mathrm{E}+03$ & 3.667 & $9.827 \mathrm{E}+01$ & 3.574 & pu240 & 2.637 \\
\hline $1000 \mathrm{yr}$ & $1.763 E+03$ & 3.111 & $5.561 \mathrm{E}+01$ & 3.010 & pu241 & 2.492 \\
\hline $2500 \mathrm{yr}$ & $7.953 \mathrm{E}+02$ & 2.609 & $2.360 \mathrm{E}+01$ & 2.560 & pu242 & 2.437 \\
\hline $5000 \mathrm{yr}$ & $6.019 \mathrm{E}+02$ & 2.678 & $1.747 \mathrm{E}+01$ & 2.597 & am241 & 2.474 \\
\hline \multirow[t]{3}{*}{$10000 \mathrm{yr}$} & $4.463 E+02$ & 2.290 & $1.276 \mathrm{E}+01$ & 2.207 & am242m & 2.156 \\
\hline & \multicolumn{2}{|c|}{ Inhalation Hazard } & \multicolumn{2}{|c|}{ Ingestion Hazard } & am243 & 12.685 \\
\hline & $\mathrm{m}^{3}$ air & $+/-\%$ & $\mathrm{~m}^{3}$ water & $+/-\%$ & cm242 & 2.155 \\
\hline Charge & $1.133 E+13$ & $\mathrm{~N} / \mathrm{A}$ & $5.380 \mathrm{E}+06$ & $\mathrm{~N} / \mathrm{A}$ & $\mathrm{cm} 244$ & 10.618 \\
\hline Discharge & $7.862 \mathrm{E}+17$ & $\mathrm{~N} / \mathrm{A}$ & $6.652 \mathrm{E}+12$ & $\mathrm{~N} / \mathrm{A}$ & $\mathrm{cm} 245$ & 9.687 \\
\hline $1 \mathrm{yr}$ & $5.685 \mathrm{E}+17$ & $\mathrm{~N} / \mathrm{A}$ & $7.698 \mathrm{E}+11$ & $\mathrm{~N} / \mathrm{A}$ & \multicolumn{2}{|c|}{ FP (+/- \%) } \\
\hline $5 \mathrm{yr}$ & $5.087 \mathrm{E}+17$ & 2.786 & $3.686 \mathrm{E}+11$ & 0.868 & c 14 & 0.483 \\
\hline $10 \mathrm{yr}$ & $5.013 E+17$ & 2.506 & $3.017 \mathrm{E}+11$ & 0.881 & se 79 & 0.408 \\
\hline $50 \mathrm{yr}$ & $4.415 \mathrm{E}+17$ & 2.710 & $1.595 \mathrm{E}+11$ & 1.439 & sr 90 & 0.436 \\
\hline $100 \mathrm{yr}$ & $3.778 \mathrm{E}+17$ & 3.100 & $9.405 E+10$ & 2.347 & tc 99 & 1.864 \\
\hline $500 \mathrm{yr}$ & $1.831 \mathrm{E}+17$ & 3.443 & $3.444 \mathrm{E}+10$ & 3.443 & i129 & 0.444 \\
\hline $1000 \mathrm{yr}$ & $1.087 \mathrm{E}+17$ & 2.906 & $2.043 E+10$ & 2.906 & cs137 & 0.413 \\
\hline $2500 \mathrm{yr}$ & $5.218 \mathrm{E}+16$ & 2.669 & $9.813 E+09$ & 2.667 & ba137m & 0.413 \\
\hline $5000 \mathrm{yr}$ & $3.979 \mathrm{E}+16$ & 2.690 & $7.488 \mathrm{E}+09$ & 2.688 & y90 & 0.436 \\
\hline $10000 \mathrm{yr}$ & $2.898 \mathrm{E}+16$ & 2.302 & $5.467 \mathrm{E}+09$ & 2.300 & cs134 & 1.166 \\
\hline \multicolumn{5}{|c|}{$\begin{array}{l}\text { Main Contributors to Uncertainty in } \\
\text { Decay Heat and Radioactivity }\end{array}$} & \multicolumn{2}{|c|}{$\begin{array}{c}\text { 1-group Cross Sections } \\
\text { Causing Uncertainty }\end{array}$} \\
\hline & \multicolumn{2}{|c|}{ Heat } & \multicolumn{2}{|c|}{ Activity } & Nuclide & Reaction \\
\hline $5 \mathrm{yr}$ & \multicolumn{2}{|c|}{$\mathrm{Cm}-244, \mathrm{Cs}-134$} & \multicolumn{2}{|c|}{ Pu241 } & Am-243 & $(\mathrm{n}, \gamma)$ \\
\hline $10 \mathrm{yr}$ & \multicolumn{2}{|c|}{$\mathrm{Cm}-244, \mathrm{Y}-90, \mathrm{Ba}-137 \mathrm{~m}$} & \multicolumn{2}{|c|}{ Pu-241 } & Pu-240 & $(\mathrm{n}, \gamma)$ \\
\hline $50 \mathrm{yr}$ & \multicolumn{2}{|c|}{ Am-241 } & \multicolumn{2}{|c|}{ Pu-241 } & $\mathrm{U}-235$ & (fission) \\
\hline $100 \mathrm{yr}$ & \multicolumn{2}{|c|}{ Am-241 } & \multicolumn{2}{|c|}{ Am-241 } & Pu-239 & (fission) \\
\hline $500 \mathrm{yr}$ & \multicolumn{2}{|c|}{ Am-241 } & \multicolumn{2}{|c|}{ Am-241 } & U-234 & $(\mathrm{n}, \gamma)$ \\
\hline $1000 \mathrm{yr}$ & \multicolumn{2}{|c|}{ Am-241 } & \multicolumn{2}{|c|}{ Am-241 } & & \\
\hline $2500 \mathrm{yr}$ & $\mathrm{Pu}-2$ & & $\mathrm{Pu}-24$ & & & \\
\hline $5000 \mathrm{yr}$ & Pu-240 & -239 & $\mathrm{Pu}-240, \mathrm{P}$ & -239 & & \\
\hline $10000 \mathrm{yr}$ & Pu-240 & -239 & $\mathrm{Pu}-240, \mathrm{P}$ & -239 & & \\
\hline
\end{tabular}

Table C.6: Results table BWR fuel burned at $50 \%$ void. 


\begin{tabular}{|c|c|c|c|c|c|c|}
\hline \multicolumn{7}{|c|}{$\begin{array}{c}\text { Results Table for BWR, 65\% Void, } 4.5 \text { w/o UOX } \\
\text { burned } 40 \text { GWD/MTU }\end{array}$} \\
\hline \multicolumn{5}{|c|}{ Uncertainty for Key Metrics } & \multirow{2}{*}{\multicolumn{2}{|c|}{$\begin{array}{c}\text { Isotopic Mass } \\
\text { Uncertainties } \\
\text { Actindes (+/- \%) }\end{array}$}} \\
\hline & \multicolumn{2}{|c|}{ Heat } & \multicolumn{2}{|c|}{ Activity } & & \\
\hline & $\mathbf{W}$ & $+/-\%$ & $\mathbf{C i}$ & $+/-\%$ & u235 & 1.677 \\
\hline Charge & $2.063 E+00$ & $\mathrm{~N} / \mathrm{A}$ & $5.817 \mathrm{E}-02$ & $\mathrm{~N} / \mathrm{A}$ & u236 & 0.839 \\
\hline Discharge & $2.346 \mathrm{E}+08$ & $\mathrm{~N} / \mathrm{A}$ & $2.449 \mathrm{E}+06$ & $\mathrm{~N} / \mathrm{A}$ & u237 & 2.479 \\
\hline $1 \mathrm{yr}$ & $2.907 \mathrm{E}+06$ & $\mathrm{~N} / \mathrm{A}$ & $1.189 \mathrm{E}+04$ & $\mathrm{~N} / \mathrm{A}$ & u238 & 0.079 \\
\hline $5 \mathrm{yr}$ & $7.286 \mathrm{E}+05$ & 1.223 & $2.243 \mathrm{E}+03$ & 1.226 & np237 & 0.697 \\
\hline $10 \mathrm{yr}$ & $5.046 \mathrm{E}+05$ & 1.232 & $1.440 \mathrm{E}+03$ & 1.175 & np239 & 14.483 \\
\hline $50 \mathrm{yr}$ & $1.613 \mathrm{E}+05$ & 1.385 & $6.824 \mathrm{E}+02$ & 0.649 & pu238 & 1.172 \\
\hline $100 \mathrm{yr}$ & $5.111 \mathrm{E}+04$ & 2.288 & $3.761 \mathrm{E}+02$ & 0.657 & pu239 & 0.920 \\
\hline $500 \mathrm{yr}$ & $3.823 E+03$ & 3.706 & $1.229 \mathrm{E}+02$ & 3.619 & pu240 & 2.536 \\
\hline $1000 \mathrm{yr}$ & $2.155 \mathrm{E}+03$ & 3.138 & $6.835 \mathrm{E}+01$ & 3.040 & pu241 & 2.479 \\
\hline $2500 \mathrm{yr}$ & $9.305 \mathrm{E}+02$ & 2.390 & $2.777 \mathrm{E}+01$ & 2.374 & pu242 & 2.531 \\
\hline $5000 \mathrm{yr}$ & $7.006 \mathrm{E}+02$ & 2.459 & $2.048 \mathrm{E}+01$ & 2.421 & am241 & 2.462 \\
\hline \multirow[t]{3}{*}{$10000 \mathrm{yr}$} & $5.253 \mathrm{E}+02$ & 2.130 & $1.518 \mathrm{E}+01$ & 2.086 & am242m & 2.154 \\
\hline & \multicolumn{2}{|c|}{ Inhalation Hazard } & \multicolumn{2}{|c|}{ Ingestion Hazard } & am243 & 14.483 \\
\hline & $\mathrm{m}^{3}$ air & $+/-\%$ & $\mathrm{~m}^{3}$ water & $+/-\%$ & cm242 & 2.153 \\
\hline Charge & $1.133 E+13$ & $\mathrm{~N} / \mathrm{A}$ & $5.380 \mathrm{E}+06$ & $\mathrm{~N} / \mathrm{A}$ & cm244 & 12.041 \\
\hline Discharge & $9.632 \mathrm{E}+17$ & $\mathrm{~N} / \mathrm{A}$ & $6.791 \mathrm{E}+12$ & $\mathrm{~N} / \mathrm{A}$ & cm245 & 10.851 \\
\hline $1 \mathrm{yr}$ & $7.212 \mathrm{E}+17$ & $\mathrm{~N} / \mathrm{A}$ & $8.098 \mathrm{E}+11$ & $\mathrm{~N} / \mathrm{A}$ & \multicolumn{2}{|c|}{ FP (+/- \%) } \\
\hline $5 \mathrm{yr}$ & $6.526 \mathrm{E}+17$ & 3.237 & $3.941 \mathrm{E}+11$ & 1.146 & c 14 & 0.544 \\
\hline $10 \mathrm{yr}$ & $6.423 \mathrm{E}+17$ & 2.862 & $3.242 \mathrm{E}+11$ & 1.150 & se 79 & 0.465 \\
\hline $50 \mathrm{yr}$ & $5.615 \mathrm{E}+17$ & 2.730 & $1.803 E+11$ & 1.624 & sr 90 & 0.496 \\
\hline $100 \mathrm{yr}$ & $4.784 \mathrm{E}+17$ & 3.106 & $1.125 \mathrm{E}+11$ & 2.489 & tc 99 & 2.238 \\
\hline $500 \mathrm{yr}$ & $2.276 \mathrm{E}+17$ & 3.482 & $4.281 \mathrm{E}+10$ & 3.482 & i129 & 0.502 \\
\hline $1000 \mathrm{yr}$ & $1.326 \mathrm{E}+17$ & 2.906 & $2.494 \mathrm{E}+10$ & 2.906 & $\operatorname{cs} 137$ & 0.473 \\
\hline $2500 \mathrm{yr}$ & $6.124 \mathrm{E}+16$ & 2.427 & $1.152 \mathrm{E}+10$ & 2.425 & ba137m & 0.473 \\
\hline $5000 \mathrm{yr}$ & $4.663 \mathrm{E}+16$ & 2.457 & $8.775 \mathrm{E}+09$ & 2.455 & y90 & 0.496 \\
\hline $10000 \mathrm{yr}$ & $3.450 \mathrm{E}+16$ & 2.131 & $6.507 \mathrm{E}+09$ & 2.129 & cs134 & 1.305 \\
\hline \multicolumn{5}{|c|}{$\begin{array}{l}\text { Main Contributors to Uncertainty in } \\
\text { Decay Heat and Radioactivity }\end{array}$} & \multicolumn{2}{|c|}{$\begin{array}{l}\text { 1-group Cross Sections } \\
\text { Causing Uncertainty }\end{array}$} \\
\hline & \multicolumn{2}{|c|}{ Heat } & \multicolumn{2}{|c|}{ Activity } & Nuclide & Reaction \\
\hline $5 \mathrm{yr}$ & \multicolumn{2}{|c|}{$\mathrm{Cm}-244, \mathrm{Cs}-134$} & \multicolumn{2}{|c|}{ Pu241 } & Am-243 & $(\mathrm{n}, \gamma)$ \\
\hline $10 \mathrm{yr}$ & \multicolumn{2}{|c|}{$\mathrm{Cm}-244, \mathrm{Y}-90, \mathrm{Ba}-137 \mathrm{~m}$} & \multicolumn{2}{|c|}{ Pu-241 } & $\mathrm{Pu}-240$ & $(\mathrm{n}, \gamma)$ \\
\hline $50 \mathrm{yr}$ & \multicolumn{2}{|c|}{ Am-241 } & \multicolumn{2}{|c|}{ Pu-241 } & $\mathrm{U}-235$ & (fission) \\
\hline $100 \mathrm{yr}$ & \multicolumn{2}{|c|}{ Am-241 } & \multicolumn{2}{|c|}{ Am-241 } & Pu-239 & (fission) \\
\hline $500 \mathrm{yr}$ & \multicolumn{2}{|c|}{ Am-241 } & \multicolumn{2}{|c|}{ Am-241 } & U-234 & $(\mathrm{n}, \gamma)$ \\
\hline $1000 \mathrm{yr}$ & \multicolumn{2}{|c|}{ Am-241 } & \multicolumn{2}{|c|}{ Am-241 } & & \\
\hline $2500 \mathrm{yr}$ & Pu-240 & -239 & $\mathrm{Pu}-240, \mathrm{Pu}$ & -239 & & \\
\hline $5000 \mathrm{yr}$ & Pu-240 & -239 & $\mathrm{Pu}-240, \mathrm{Pu}$ & -239 & & \\
\hline $10000 \mathrm{yr}$ & Pu-240 & -239 & $\mathrm{Pu}-240, \mathrm{Pu}$ & -239 & & \\
\hline
\end{tabular}

Table C.7: Results table for BWR fuel burned at $65 \%$ void. 


\begin{tabular}{|c|c|c|c|c|c|c|}
\hline \multicolumn{7}{|c|}{ Results Table for PWR, MOX fuel burned 50 GWD/MTU } \\
\hline \multicolumn{5}{|c|}{ Uncertainty for Key Metrics } & \multirow{2}{*}{\multicolumn{2}{|c|}{\begin{tabular}{|c|} 
Isotopic Mass Uncertainties \\
Actindes (+/- \%)
\end{tabular}}} \\
\hline & \multicolumn{2}{|c|}{ Heat } & \multicolumn{2}{|c|}{ Activity } & & \\
\hline & $\mathbf{W}$ & $+1-\%$ & $\mathbf{C i}$ & $+/-\%$ & u235 & 1.367 \\
\hline Charge & $9.887 \mathrm{E}+05$ & $\mathrm{~N} / \mathrm{A}$ & $3.926 \mathrm{E}+03$ & $\mathrm{~N} / \mathrm{A}$ & $\mathrm{u} 236$ & 1.037 \\
\hline Discharge & $2.888 \mathrm{E}+08$ & $\mathrm{~N} / \mathrm{A}$ & $2.990 \mathrm{E}+06$ & $\mathrm{~N} / \mathrm{A}$ & u237 & 1.929 \\
\hline $1 \mathrm{yr}$ & $4.927 \mathrm{E}+06$ & $\mathrm{~N} / \mathrm{A}$ & $2.543 E+04$ & $\mathrm{~N} / \mathrm{A}$ & u238 & 0.092 \\
\hline $5 \mathrm{yr}$ & $1.602 \mathrm{E}+06$ & 11.670 & $7.824 \mathrm{E}+03$ & 2.723 & np237 & 0.790 \\
\hline $10 \mathrm{yr}$ & $1.157 \mathrm{E}+06$ & 11.020 & $6.267 \mathrm{E}+03$ & 2.691 & np239 & 24.978 \\
\hline $50 \mathrm{yr}$ & $3.288 \mathrm{E}+05$ & 4.158 & $3.765 \mathrm{E}+03$ & 1.744 & pu238 & 1.312 \\
\hline $100 \mathrm{yr}$ & $1.255 \mathrm{E}+05$ & 2.058 & $2.567 \mathrm{E}+03$ & 1.325 & pu239 & 1.384 \\
\hline $500 \mathrm{yr}$ & $2.354 \mathrm{E}+04$ & 2.743 & $7.581 \mathrm{E}+02$ & 2.777 & pu240 & 2.361 \\
\hline $1000 \mathrm{yr}$ & $1.287 \mathrm{E}+04$ & 2.803 & $4.055 E+02$ & 3.009 & pu241 & 1.929 \\
\hline $2500 \mathrm{yr}$ & $5.661 \mathrm{E}+03$ & 3.832 & $1.679 \mathrm{E}+02$ & 4.423 & pu242 & 2.590 \\
\hline 5000 yr & $4.153 E+03$ & 4.149 & $1.212 \mathrm{E}+02$ & 4.742 & am241 & 1.838 \\
\hline \multirow[t]{3}{*}{$10000 \mathrm{yr}$} & $2.909 \mathrm{E}+03$ & 3.725 & $8.490 \mathrm{E}+01$ & 4.263 & am242m & 1.213 \\
\hline & \multicolumn{2}{|c|}{ Inhalation Hazard } & \multicolumn{2}{|c|}{ Ingestion Hazard } & $\operatorname{am} 243$ & 24.978 \\
\hline & $\mathrm{m}^{3}$ air & $+/-\%$ & $\mathrm{~m}^{3}$ water & $+l-\%$ & cm242 & 1.213 \\
\hline Charge & $9.004 \mathrm{E}+18$ & $\mathrm{~N} / \mathrm{A}$ & $1.701 \mathrm{E}+12$ & $\mathrm{~N} / \mathrm{A}$ & cm244 & 17.637 \\
\hline Discharge & $1.156 \mathrm{E}+19$ & $\mathrm{~N} / \mathrm{A}$ & $1.021 \mathrm{E}+13$ & $\mathrm{~N} / \mathrm{A}$ & cm245 & 14.425 \\
\hline $1 \mathrm{yr}$ & $1.015 \mathrm{E}+19$ & $\mathrm{~N} / \mathrm{A}$ & $2.783 E+12$ & $\mathrm{~N} / \mathrm{A}$ & \multicolumn{2}{|c|}{ FP (+/- \%) } \\
\hline $5 \mathrm{yr}$ & $9.341 \mathrm{E}+18$ & 8.639 & $2.037 \mathrm{E}+12$ & 7.608 & c 14 & 0.837 \\
\hline $10 \mathrm{yr}$ & $8.825 \mathrm{E}+18$ & 7.588 & $1.852 \mathrm{E}+12$ & 6.863 & se 79 & 0.734 \\
\hline $50 \mathrm{yr}$ & $6.207 \mathrm{E}+18$ & 2.906 & $1.238 \mathrm{E}+12$ & 2.758 & sr 90 & 0.690 \\
\hline $100 \mathrm{yr}$ & $4.600 \mathrm{E}+18$ & 1.989 & $8.878 \mathrm{E}+11$ & 1.944 & tc 99 & 2.561 \\
\hline $500 \mathrm{yr}$ & $1.410 \mathrm{E}+18$ & 2.612 & $2.652 \mathrm{E}+11$ & 2.611 & i129 & 0.764 \\
\hline $1000 \mathrm{yr}$ & $7.853 \mathrm{E}+17$ & 2.697 & $1.477 \mathrm{E}+11$ & 2.696 & cs137 & 0.759 \\
\hline $2500 \mathrm{yr}$ & $3.658 \mathrm{E}+17$ & 3.576 & $6.884 \mathrm{E}+10$ & 3.573 & ba137m & 0.759 \\
\hline $5000 \mathrm{yr}$ & $2.717 \mathrm{E}+17$ & 3.763 & $5.121 \mathrm{E}+10$ & 3.761 & y90 & 0.690 \\
\hline $10000 \mathrm{yr}$ & $1.895 \mathrm{E}+17$ & 3.386 & $3.591 \mathrm{E}+10$ & 3.383 & cs134 & 1.673 \\
\hline \multicolumn{5}{|c|}{$\begin{array}{l}\text { Main Contributors to Uncertainty in } \\
\text { Decay Heat and Radioactivity }\end{array}$} & \multicolumn{2}{|c|}{$\begin{array}{c}\text { 1-group Cross Sections } \\
\text { Causing Uncertainty }\end{array}$} \\
\hline & \multicolumn{2}{|c|}{ Heat } & \multicolumn{2}{|c|}{ Activity } & Nuclide & Reaction \\
\hline $5 \mathrm{yr}$ & \multicolumn{2}{|c|}{$\mathrm{Cm}-244$} & \multicolumn{2}{|c|}{ Pu-241, Cm-244 } & $\mathrm{Am}-243$ & $(\mathrm{n}, \gamma)$ \\
\hline $10 \mathrm{yr}$ & \multicolumn{2}{|c|}{$\mathrm{Cm}-244$} & \multicolumn{2}{|c|}{$\mathrm{Pu}-241, \mathrm{Cm}-244$} & Pu-239 & (fission) \\
\hline $50 \mathrm{yr}$ & \multicolumn{2}{|c|}{$\mathrm{Cm}-244$} & \multicolumn{2}{|c|}{$\mathrm{Pu}-241, \mathrm{Cm}-244$} & $\mathrm{Pu}-240$ & (fission) \\
\hline $100 \mathrm{yr}$ & \multicolumn{2}{|c|}{ Pu-238, Am-241, Cm-244 } & \multicolumn{2}{|c|}{ Pu-238, Am-241, Cm-244 } & Pu-242 & $(\mathrm{n}, \gamma)$ \\
\hline $500 \mathrm{yr}$ & \multicolumn{2}{|c|}{ Am-241 } & \multicolumn{2}{|c|}{ Am-241 } & $\mathrm{Pu}-240$ & $(\mathrm{n}, \gamma)$ \\
\hline $1000 \mathrm{yr}$ & \multicolumn{2}{|c|}{ Am-241, Am-243 } & \multicolumn{2}{|c|}{ Am-241, Am-243 } & & \\
\hline $2500 \mathrm{yr}$ & $\mathrm{Pu}-240$ & 243 & $\mathrm{Pu}-240, \mathrm{~A}$ & 243 & & \\
\hline $5000 \mathrm{yr}$ & Pu-240, & 243 & $\mathrm{Pu}-240, \mathrm{~A}$ & 243 & & \\
\hline $10000 \mathrm{yr}$ & Pu-240, & 243 & Pu-240, A & 243 & & \\
\hline
\end{tabular}

Table C.8: Results table for clean MOX fuel. 


\begin{tabular}{|c|c|c|c|c|c|c|}
\hline \multicolumn{7}{|c|}{$\begin{array}{r}\text { Results Table for PWR, MOX f } \\
\text { GWD }\end{array}$} \\
\hline \multicolumn{5}{|c|}{ Uncertainty for Key Metrics } & \multirow{2}{*}{\multicolumn{2}{|c|}{\begin{tabular}{|c|} 
ssotopic Mass Uncertainties \\
Actindes (+/- \%) \\
\end{tabular}}} \\
\hline 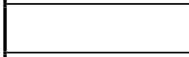 & \multicolumn{2}{|c|}{ Heat } & \multicolumn{2}{|c|}{ Activity } & & \\
\hline & W & $+/-\%$ & $\mathbf{C i}$ & $+/-\%$ & u235 & 1.354 \\
\hline Charge & $1.040 \mathrm{E}+06$ & $\mathrm{~N} / \mathrm{A}$ & $5.643 E+03$ & $\mathrm{~N} / \mathrm{A}$ & u236 & 1.059 \\
\hline Discharge & $2.977 \mathrm{E}+08$ & $\mathrm{~N} / \mathrm{A}$ & $3.134 \mathrm{E}+06$ & $\mathrm{~N} / \mathrm{A}$ & u237 & 1.863 \\
\hline $1 \mathrm{yr}$ & $5.927 \mathrm{E}+06$ & $\mathrm{~N} / \mathrm{A}$ & $6.168 \mathrm{E}+04$ & $\mathrm{~N} / \mathrm{A}$ & u238 & 0.096 \\
\hline $5 \mathrm{yr}$ & $1.796 \mathrm{E}+06$ & 6.146 & $1.419 \mathrm{E}+04$ & 2.353 & np237 & 0.373 \\
\hline $10 \mathrm{yr}$ & $1.343 \mathrm{E}+06$ & 5.588 & $1.232 \mathrm{E}+04$ & 2.281 & np239 & 23.074 \\
\hline $50 \mathrm{yr}$ & 4.637E+05 & 2.103 & $8.179 \mathrm{E}+03$ & 1.316 & pu238 & 0.791 \\
\hline $100 \mathrm{yr}$ & $2.200 \mathrm{E}+05$ & 1.265 & $5.641 \mathrm{E}+03$ & 1.009 & pu239 & 1.354 \\
\hline $500 \mathrm{yr}$ & $3.481 \mathrm{E}+04$ & 1.741 & $1.120 \mathrm{E}+03$ & 1.788 & pu240 & 2.299 \\
\hline $1000 \mathrm{yr}$ & $1.656 \mathrm{E}+04$ & 2.095 & $5.249 \mathrm{E}+02$ & 2.310 & pu241 & 1.863 \\
\hline $2500 \mathrm{yr}$ & $6.460 \mathrm{E}+03$ & 3.484 & $1.917 \mathrm{E}+02$ & 4.053 & pu242 & 2.393 \\
\hline $5000 \mathrm{yr}$ & $4.640 \mathrm{E}+03$ & 3.893 & $1.349 \mathrm{E}+02$ & 4.459 & am241 & 1.183 \\
\hline \multirow[t]{3}{*}{$10000 \mathrm{yr}$} & $3.332 \mathrm{E}+03$ & 3.457 & $9.670 \mathrm{E}+01$ & 3.955 & am242m & 1.162 \\
\hline & \multicolumn{2}{|c|}{ Inhalation Hazard } & \multicolumn{2}{|c|}{ Ingestion Hazard } & $\operatorname{am} 243$ & 23.074 \\
\hline & $\mathrm{m}^{3}$ air & $+/-\%$ & $\mathrm{~m}^{3}$ water & $+/-\%$ & $\mathrm{~cm} 242$ & 1.162 \\
\hline Charge & $1.196 \mathrm{E}+19$ & $\mathrm{~N} / \mathrm{A}$ & $2.257 \mathrm{E}+12$ & $\mathrm{~N} / \mathrm{A}$ & $\mathrm{cm} 244$ & 16.130 \\
\hline Discharge & $3.657 \mathrm{E}+19$ & $\mathrm{~N} / \mathrm{A}$ & $1.502 \mathrm{E}+13$ & $\mathrm{~N} / \mathrm{A}$ & cm245 & 13.127 \\
\hline $1 \mathrm{yr}$ & $2.528 \mathrm{E}+19$ & $\mathrm{~N} / \mathrm{A}$ & $5.618 \mathrm{E}+12$ & $\mathrm{~N} / \mathrm{A}$ & \multicolumn{2}{|c|}{ FP (+/- \%) } \\
\hline $5 \mathrm{yr}$ & $2.145 E+19$ & 3.866 & $4.323 E+12$ & 3.659 & c 14 & 0.946 \\
\hline $10 \mathrm{yr}$ & $2.046 \mathrm{E}+19$ & 3.401 & $4.049 \mathrm{E}+12$ & 3.259 & se 79 & 0.816 \\
\hline $50 \mathrm{yr}$ & $1.477 \mathrm{E}+19$ & 1.565 & $2.856 \mathrm{E}+12$ & 1.531 & sr 90 & 0.764 \\
\hline $100 \mathrm{yr}$ & 1.057E+19 & 1.256 & $2.016 \mathrm{E}+12$ & 1.244 & tc 99 & 2.942 \\
\hline $500 \mathrm{yr}$ & $2.069 \mathrm{E}+18$ & 1.681 & $3.895 \mathrm{E}+11$ & 1.679 & i129 & 0.828 \\
\hline $1000 \mathrm{yr}$ & $9.956 \mathrm{E}+17$ & 2.074 & $1.874 \mathrm{E}+11$ & 2.072 & cs137 & 0.819 \\
\hline $2500 \mathrm{yr}$ & $4.101 \mathrm{E}+17$ & 3.294 & $7.734 \mathrm{E}+10$ & 3.288 & ba137m & 0.819 \\
\hline $5000 \mathrm{yr}$ & $2.976 \mathrm{E}+17$ & 3.564 & $5.637 \mathrm{E}+10$ & 3.557 & y90 & 0.764 \\
\hline $10000 \mathrm{yr}$ & $2.104 \mathrm{E}+17$ & 3.194 & $4.041 \mathrm{E}+10$ & 3.189 & cs134 & 1.682 \\
\hline \multicolumn{5}{|c|}{$\begin{array}{l}\text { Main Contributors to Uncertainty in } \\
\text { Decay Heat and Radioactivity }\end{array}$} & \multicolumn{2}{|c|}{$\begin{array}{c}\text { 1-group Cross Sections } \\
\text { Causing Uncertainty }\end{array}$} \\
\hline & \multicolumn{2}{|c|}{ Heat } & \multicolumn{2}{|c|}{ Activity } & Nuclide & Reaction \\
\hline $5 \mathrm{yr}$ & \multicolumn{2}{|c|}{$\mathrm{Cm}-244$} & \multicolumn{2}{|c|}{$\mathrm{Pu}-241, \mathrm{Cm}-244$} & Am-243 & $(\mathrm{n}, \gamma)$ \\
\hline $10 \mathrm{yr}$ & \multicolumn{2}{|c|}{$\mathrm{Cm}-244$} & \multicolumn{2}{|c|}{ Pu-241, Cm-244 } & Pu-239 & (fission) \\
\hline $50 \mathrm{yr}$ & \multicolumn{2}{|c|}{$\mathrm{Cm}-244$} & \multicolumn{2}{|c|}{ Pu-241, Cm-244 } & Pu-242 & $(\mathrm{n}, \gamma)$ \\
\hline $100 \mathrm{yr}$ & \multicolumn{2}{|c|}{ Pu-238, Am-241 } & \multicolumn{2}{|c|}{ Pu-238, Am-241 } & Pu-241 & (fission) \\
\hline $500 \mathrm{yr}$ & \multicolumn{2}{|c|}{ Am-241 } & \multicolumn{2}{|c|}{ Am-241 } & Pu-240 & $(\mathrm{n}, \gamma)$ \\
\hline $1000 \mathrm{yr}$ & \multicolumn{2}{|c|}{ Am-241, Am-243 } & \multicolumn{2}{|c|}{ Am-241, Am-243 } & & \\
\hline $2500 \mathrm{yr}$ & $\mathrm{Pu}-240, \mathrm{~A}$ & 243 & $\mathrm{Pu}-240, \mathrm{~A}$ & 243 & & \\
\hline $5000 \mathrm{yr}$ & $\mathrm{Pu}-240, \mathrm{~A}$ & 243 & $\mathrm{Pu}-240, \mathrm{~A}$ & 243 & & \\
\hline $10000 \mathrm{yr}$ & $\mathrm{Pu}-240, \mathrm{~A}$ & 243 & $\mathrm{Pu}-240, \mathrm{~A}$ & 243 & & \\
\hline
\end{tabular}

Table C.9: Results table for MOX fuel with impurities. 


\begin{tabular}{|c|c|c|c|c|c|c|}
\hline \multicolumn{7}{|c|}{ Results Table for PWR, 4.5 w/c } \\
\hline \multicolumn{5}{|c|}{ Uncertainty for Key Metrics } & \multirow{2}{*}{\multicolumn{2}{|c|}{$\begin{array}{c}\begin{array}{c}\text { Isotopic Mass } \\
\text { Uncertainties }\end{array} \\
\text { Actindes (+/- \%) }\end{array}$}} \\
\hline 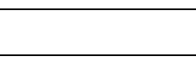 & \multicolumn{2}{|c|}{ Heat } & \multicolumn{2}{|c|}{ Activity } & & \\
\hline & $\mathbf{W}$ & $+/-\%$ & $\mathbf{C i}$ & $+/-\%$ & u235 & 0.620 \\
\hline Charge & 6.096E-02 & $\mathrm{N} / \mathrm{A}$ & $2.160 \mathrm{E}+00$ & $\mathrm{~N} / \mathrm{A}$ & u236 & 1.052 \\
\hline Discharge & $6.036 \mathrm{E}+04$ & $\mathrm{~N} / \mathrm{A}$ & $2.249 \mathrm{E}+07$ & $\mathrm{~N} / \mathrm{A}$ & $\mathbf{u} 237$ & 2.067 \\
\hline $1 \mathrm{yr}$ & $4.050 \mathrm{E}+03$ & $\mathrm{~N} / \mathrm{A}$ & $8.538 \mathrm{E}+05$ & $\mathrm{~N} / \mathrm{A}$ & u238 & 0.015 \\
\hline $5 \mathrm{yr}$ & $2.156 \mathrm{E}+03$ & 0.696 & $6.427 \mathrm{E}+05$ & 0.807 & np237 & 1.609 \\
\hline $10 \mathrm{yr}$ & $1.638 \mathrm{E}+03$ & 0.779 & $5.280 \mathrm{E}+05$ & 0.771 & np239 & 3.878 \\
\hline $50 \mathrm{yr}$ & $7.799 \mathrm{E}+02$ & 1.020 & $1.825 \mathrm{E}+05$ & 0.352 & pu238 & 1.749 \\
\hline $100 \mathrm{yr}$ & $4.090 \mathrm{E}+02$ & 1.736 & $5.788 \mathrm{E}+04$ & 0.384 & pu239 & 1.065 \\
\hline $500 \mathrm{yr}$ & $1.205 \mathrm{E}+02$ & 2.990 & $3.737 \mathrm{E}+03$ & 2.906 & pu240 & 2.486 \\
\hline $1000 \mathrm{yr}$ & $6.878 \mathrm{E}+01$ & 2.616 & $2.177 \mathrm{E}+03$ & 2.525 & pu241 & 2.065 \\
\hline $2500 \mathrm{yr}$ & $3.045 \mathrm{E}+01$ & 2.577 & $1.018 \mathrm{E}+03$ & 2.481 & pu242 & 3.897 \\
\hline $5000 \mathrm{yr}$ & $2.263 \mathrm{E}+01$ & 2.672 & $7.679 \mathrm{E}+02$ & 2.539 & am241 & 2.063 \\
\hline \multirow[t]{3}{*}{$10000 \mathrm{yr}$} & $1.625 \mathrm{E}+01$ & 2.343 & $5.551 \mathrm{E}+02$ & 2.212 & am242m & 2.121 \\
\hline & \multicolumn{2}{|c|}{ Inhalation Hazard } & \multicolumn{2}{|c|}{ Ingestion Hazard } & am243 & 3.884 \\
\hline & $\mathrm{m}^{3}$ air & $+1-\%$ & $\mathrm{~m}^{3}$ water & $+/-\%$ & cm242 & 2.121 \\
\hline Charge & $1.188 \mathrm{E}+13$ & $\mathrm{~N} / \mathrm{A}$ & $5.636 \mathrm{E}+06$ & $\mathrm{~N} / \mathrm{A}$ & $\mathrm{cm} 244$ & 4.337 \\
\hline Discharge & $1.051 \mathrm{E}+18$ & $\mathrm{~N} / \mathrm{A}$ & $1.654 \mathrm{E}+12$ & $\mathrm{~N} / \mathrm{A}$ & cm245 & 4.673 \\
\hline $1 \mathrm{yr}$ & $8.750 \mathrm{E}+17$ & $\mathrm{~N} / \mathrm{A}$ & $6.188 \mathrm{E}+11$ & $\mathrm{~N} / \mathrm{A}$ & \multicolumn{2}{|c|}{ FP (+/- \%) } \\
\hline $5 \mathrm{yr}$ & $8.076 \mathrm{E}+17$ & 2.334 & $4.527 \mathrm{E}+11$ & 0.794 & c 14 & 0.360 \\
\hline $10 \mathrm{yr}$ & $7.801 \mathrm{E}+17$ & 2.178 & $3.819 \mathrm{E}+11$ & 0.845 & se 79 & 0.087 \\
\hline $50 \mathrm{yr}$ & $6.216 \mathrm{E}+17$ & 2.233 & $2.036 \mathrm{E}+11$ & 1.285 & sr 90 & 0.120 \\
\hline $100 \mathrm{yr}$ & $5.075 \mathrm{E}+17$ & 2.476 & $1.216 \mathrm{E}+11$ & 1.945 & tc 99 & 0.076 \\
\hline $500 \mathrm{yr}$ & $2.257 \mathrm{E}+17$ & 2.824 & $4.246 \mathrm{E}+10$ & 2.823 & i129 & 0.174 \\
\hline $1000 \mathrm{yr}$ & $1.352 \mathrm{E}+17$ & 2.509 & $2.542 \mathrm{E}+10$ & 2.508 & cs137 & 0.025 \\
\hline $2500 \mathrm{yr}$ & $6.736 \mathrm{E}+16$ & 2.668 & $1.266 \mathrm{E}+10$ & 2.665 & ba137m & 0.025 \\
\hline $5000 \mathrm{yr}$ & $5.144 \mathrm{E}+16$ & 2.711 & $9.671 \mathrm{E}+09$ & 2.708 & y90 & 0.120 \\
\hline $10000 \mathrm{yr}$ & $3.693 \mathrm{E}+16$ & 2.378 & $6.944 \mathrm{E}+09$ & 2.376 & cs134 & 0.318 \\
\hline \multirow{2}{*}{\multicolumn{5}{|c|}{$\begin{array}{l}\text { Main Contributors to Uncertainty in } \\
\text { Decay Heat and Radioactivity }\end{array}$}} & \multicolumn{2}{|c|}{ k-effective Values } \\
\hline & & & & & BOL & 1.4181 \\
\hline & \multicolumn{2}{|c|}{ Heat } & \multicolumn{2}{|c|}{ Activity } & EOL & 0.9696 \\
\hline $5 \mathrm{yr}$ & \multicolumn{2}{|c|}{$\mathrm{Cm}-244$} & \multicolumn{2}{|c|}{ Pu241 } & & \\
\hline $10 \mathrm{yr}$ & \multicolumn{2}{|c|}{ Pu-238, Cm-244 } & \multicolumn{2}{|c|}{ Pu-241 } & & \\
\hline 50 yr & \multicolumn{2}{|c|}{$\mathrm{Pu}-238, \mathrm{Am}-241$} & \multicolumn{2}{|c|}{ Pu-241 } & & \\
\hline $100 \mathrm{yr}$ & \multicolumn{2}{|c|}{$\mathrm{Am}-241$} & \multicolumn{2}{|c|}{ Am-241 } & & \\
\hline $500 \mathrm{yr}$ & \multicolumn{2}{|c|}{ Am-241 } & \multicolumn{2}{|c|}{ Am-241 } & & \\
\hline $1000 \mathrm{yr}$ & \multicolumn{2}{|c|}{ Pu-240, Am-241 } & \multicolumn{2}{|c|}{ Pu-240, Am-241 } & & \\
\hline $2500 \mathrm{yr}$ & $\mathrm{Pu}-240 \mathrm{PL}$ & -239 & $\mathrm{Pu}-240, \mathrm{P}$ & -239 & & \\
\hline $5000 \mathrm{yr}$ & $\mathrm{Pu}-240 \mathrm{Pu}$ & -239 & $\mathrm{Pu}-240, \mathrm{P}$ & -239 & & \\
\hline $10000 \mathrm{yr}$ & $\mathrm{Pu}-240 \mathrm{Pu}$ & -239 & $\mathrm{Pu}-240, \mathrm{P}$ & -239 & & \\
\hline
\end{tabular}

Table C.10: Results table for TRITON PWR model, 48 GWD/MTU 


\begin{tabular}{|c|c|c|c|c|c|c|}
\hline \multicolumn{7}{|c|}{ Results Table for $F R, C R=0$} \\
\hline \multicolumn{5}{|c|}{ Uncertainty for Key Metrics } & \multicolumn{2}{|c|}{$\begin{array}{l}\text { Isotopic Mass } \\
\text { Uncertainties }\end{array}$} \\
\hline & \multicolumn{2}{|c|}{ Heat } & \multicolumn{2}{|c|}{ Activity } & \multicolumn{2}{|c|}{ Actindes (+/- \%) } \\
\hline & $\mathbf{W}$ & $+/-\%$ & $\mathbf{C i}$ & $+/-\%$ & u235 & 1.490 \\
\hline Charge & $6.397 \mathrm{E}+04$ & $\mathrm{~N} / \mathrm{A}$ & $5.520 \mathrm{E}+06$ & $\mathrm{~N} / \mathrm{A}$ & u236 & 1.795 \\
\hline Discharge & $3.748 \mathrm{E}+05$ & $\mathrm{~N} / \mathrm{A}$ & $3.982 \mathrm{E}+07$ & $\mathrm{~N} / \mathrm{A}$ & u237 & 2.764 \\
\hline $1 \mathrm{yr}$ & $8.308 \mathrm{E}+04$ & $\mathrm{~N} / \mathrm{A}$ & $6.625 \mathrm{E}+06$ & $\mathrm{~N} / \mathrm{A}$ & u238 & 0.205 \\
\hline $5 \mathrm{yr}$ & $5.399 \mathrm{E}+04$ & 14.160 & $5.170 \mathrm{E}+06$ & 5.200 & np237 & 1.114 \\
\hline $10 \mathrm{yr}$ & $4.820 \mathrm{E}+04$ & 13.142 & $4.290 \mathrm{E}+06$ & 5.104 & np239 & 10.752 \\
\hline $50 \mathrm{yr}$ & $2.555 E+04$ & 6.068 & $1.339 E+06$ & 3.610 & pu238 & 3.371 \\
\hline $100 \mathrm{yr}$ & $1.677 \mathrm{E}+04$ & 3.388 & $6.221 \mathrm{E}+05$ & 2.753 & pu239 & 1.468 \\
\hline $500 \mathrm{yr}$ & $5.375 E+03$ & 1.732 & $1.702 \mathrm{E}+05$ & 1.708 & pu240 & 0.906 \\
\hline $1000 \mathrm{yr}$ & $3.142 \mathrm{E}+03$ & 1.581 & $1.014 \mathrm{E}+05$ & 1.652 & pu241 & 2.764 \\
\hline $2500 \mathrm{yr}$ & $1.639 \mathrm{E}+03$ & 1.768 & $5.534 \mathrm{E}+04$ & 1.978 & pu242 & 0.548 \\
\hline $5000 \mathrm{yr}$ & $1.218 \mathrm{E}+03$ & 1.867 & $4.150 \mathrm{E}+04$ & 2.076 & am241 & 1.341 \\
\hline \multirow[t]{3}{*}{$10000 \mathrm{yr}$} & $8.012 \mathrm{E}+02$ & 1.817 & $2.728 \mathrm{E}+04$ & 2.009 & am242m & 1.434 \\
\hline & \multicolumn{2}{|c|}{ Inhalation Hazard } & \multicolumn{2}{|c|}{ Ingestion Hazard } & am243 & 10.752 \\
\hline & $\mathrm{m}^{3}$ air & $+/-\%$ & $\mathrm{~m}^{3}$ water & $+/-\%$ & cm242 & 1.434 \\
\hline Charge & $7.719 \mathrm{E}+19$ & $\mathrm{~N} / \mathrm{A}$ & $1.458 \mathrm{E}+13$ & $\mathrm{~N} / \mathrm{A}$ & cm244 & 13.025 \\
\hline Discharge & $1.044 \mathrm{E}+20$ & $\mathrm{~N} / \mathrm{A}$ & $2.136 \mathrm{E}+13$ & $\mathrm{~N} / \mathrm{A}$ & cm245 & 4.344 \\
\hline $1 \mathrm{yr}$ & $8.267 \mathrm{E}+19$ & $\mathrm{~N} / \mathrm{A}$ & $1.612 \mathrm{E}+13$ & $\mathrm{~N} / \mathrm{A}$ & \multicolumn{2}{|c|}{ FP (+/- \%) } \\
\hline $5 \mathrm{yr}$ & $7.580 \mathrm{E}+19$ & 10.165 & $1.471 \mathrm{E}+13$ & 9.930 & c 14 & 1.655 \\
\hline $10 \mathrm{yr}$ & $6.983 E+19$ & 9.232 & $1.350 \mathrm{E}+13$ & 9.048 & se 79 & 1.137 \\
\hline $50 \mathrm{yr}$ & $4.339 \mathrm{E}+19$ & 4.610 & $8.305 E+12$ & 4.559 & sr 90 & 0.348 \\
\hline $100 \mathrm{yr}$ & $3.108 E+19$ & 3.422 & $5.895 \mathrm{E}+12$ & 3.410 & tc 99 & 0.319 \\
\hline $500 \mathrm{yr}$ & $1.033 \mathrm{E}+19$ & 1.623 & $1.944 \mathrm{E}+12$ & 1.623 & i129 & 0.412 \\
\hline $1000 \mathrm{yr}$ & $6.330 \mathrm{E}+18$ & 1.486 & $1.190 \mathrm{E}+12$ & 1.485 & cs137 & 0.094 \\
\hline $2500 \mathrm{yr}$ & $3.619 \mathrm{E}+18$ & 1.664 & $6.805 \mathrm{E}+11$ & 1.663 & ba137m & 0.094 \\
\hline $5000 \mathrm{yr}$ & $2.735 \mathrm{E}+18$ & 1.733 & $5.143 \mathrm{E}+11$ & 1.732 & y90 & 0.348 \\
\hline $10000 \mathrm{yr}$ & $1.797 \mathrm{E}+18$ & 1.698 & $3.379 \mathrm{E}+11$ & 1.697 & cs134 & 2.553 \\
\hline \multirow{2}{*}{\multicolumn{5}{|c|}{$\begin{array}{l}\text { Main Contributors to Uncertainty in } \\
\text { Decay Heat and Radioactivity }\end{array}$}} & \multicolumn{2}{|c|}{ k-effective Values } \\
\hline & & & & & BOL & 1.2856 \\
\hline & \multicolumn{2}{|c|}{ Heat } & \multicolumn{2}{|c|}{ Activity } & EOL & 1.1792 \\
\hline $5 \mathrm{yr}$ & \multicolumn{2}{|c|}{$\mathrm{Cm}-244$} & \multicolumn{2}{|c|}{ Cm-244, Pu-241 } & & \\
\hline $10 \mathrm{yr}$ & \multicolumn{2}{|c|}{$\mathrm{Cm}-244$} & \multicolumn{2}{|c|}{$\mathrm{Cm}-244, \mathrm{Pu}-241$} & & \\
\hline $50 \mathrm{yr}$ & \multicolumn{2}{|c|}{$\mathrm{Cm}-244, \mathrm{Pu}-238$} & \multicolumn{2}{|c|}{$\mathrm{Cm}-244, \mathrm{Pu}-238$} & & \\
\hline $100 \mathrm{yr}$ & \multicolumn{2}{|c|}{ Pu-238, Am-241 } & \multicolumn{2}{|c|}{ Pu-238, Am-241 } & & \\
\hline $500 \mathrm{yr}$ & \multicolumn{2}{|c|}{$\mathrm{Am}-241$} & \multicolumn{2}{|c|}{$\mathrm{Am}-241$} & & \\
\hline $1000 \mathrm{yr}$ & \multicolumn{2}{|c|}{ Am-241 } & Am-24 & & & \\
\hline $2500 \mathrm{yr}$ & Am-243, & $1-240$ & Am-243, P & $1-240$ & & \\
\hline $5000 \mathrm{yr}$ & Am-243, & $1-240$ & Am-243, P & $1-240$ & & \\
\hline $10000 \mathrm{yr}$ & $\begin{array}{r}\mathrm{Am}-243, \mathrm{Pu} \\
24 \mathrm{C}\end{array}$ & $39, \mathrm{Pu}-$ & $\begin{array}{r}\mathrm{Am}-243, \mathrm{Pu}- \\
240 \\
\end{array}$ & 39, Pu- & & \\
\hline
\end{tabular}

Table C.11: Results table for fast reactor fuel of $\mathrm{CR}=\mathbf{0 . 2 5}$. 


\begin{tabular}{|c|c|c|c|c|c|c|}
\hline \multicolumn{7}{|c|}{ Results Table for $F R, C R=0.7$} \\
\hline \multicolumn{5}{|c|}{ Uncertainty for Key Metrics } & \multicolumn{2}{|c|}{$\begin{array}{l}\text { Isotopic Mass } \\
\text { Uncertainties }\end{array}$} \\
\hline & \multicolumn{2}{|c|}{ Heat } & \multicolumn{2}{|c|}{ Activity } & \multicolumn{2}{|c|}{ Actindes (+/- \%) } \\
\hline & $\mathbf{W}$ & $+/-\%$ & $\mathbf{C i}$ & $+/-\%$ & u235 & 2.587 \\
\hline Charge & $9.086 \mathrm{E}+03$ & $\mathrm{~N} / \mathrm{A}$ & $1.138 \mathrm{E}+06$ & $\mathrm{~N} / \mathrm{A}$ & u236 & 4.793 \\
\hline Discharge & $1.300 \mathrm{E}+05$ & $\mathrm{~N} / \mathrm{A}$ & $3.333 \mathrm{E}+07$ & $\mathrm{~N} / \mathrm{A}$ & u237 & 3.832 \\
\hline $1 \mathrm{yr}$ & $1.763 \mathrm{E}+04$ & $\mathrm{~N} / \mathrm{A}$ & $2.142 \mathrm{E}+06$ & $\mathrm{~N} / \mathrm{A}$ & u238 & 0.230 \\
\hline $5 \mathrm{yr}$ & $1.208 \mathrm{E}+04$ & 14.673 & $1.729 \mathrm{E}+06$ & 4.552 & np237 & 1.680 \\
\hline $10 \mathrm{yr}$ & $1.068 \mathrm{E}+04$ & 13.774 & $1.434 \mathrm{E}+06$ & 4.416 & np239 & 15.475 \\
\hline $50 \mathrm{yr}$ & $6.002 \mathrm{E}+03$ & 6.423 & $4.625 \mathrm{E}+05$ & 2.845 & pu238 & 4.824 \\
\hline $100 \mathrm{yr}$ & $4.051 \mathrm{E}+03$ & 4.192 & $1.920 \mathrm{E}+05$ & 2.680 & pu239 & 2.406 \\
\hline $500 \mathrm{yr}$ & $1.502 \mathrm{E}+03$ & 2.777 & $4.738 \mathrm{E}+04$ & 2.710 & pu240 & 1.992 \\
\hline $1000 \mathrm{yr}$ & $9.631 \mathrm{E}+02$ & 2.588 & $3.085 \mathrm{E}+04$ & 2.584 & pu241 & 3.833 \\
\hline $2500 \mathrm{yr}$ & $5.749 \mathrm{E}+02$ & 2.903 & $1.896 \mathrm{E}+04$ & 2.933 & pu242 & 1.150 \\
\hline $5000 \mathrm{yr}$ & $4.473 \mathrm{E}+02$ & 3.009 & $1.482 \mathrm{E}+04$ & 3.024 & am241 & 2.369 \\
\hline \multirow[t]{3}{*}{$10000 \mathrm{yr}$} & $3.094 \mathrm{E}+02$ & 2.993 & $1.024 \mathrm{E}+04$ & 2.993 & am242m & 1.836 \\
\hline & \multicolumn{2}{|c|}{ Inhalation Hazard } & \multicolumn{2}{|c|}{ Ingestion Hazard } & am243 & 15.475 \\
\hline & $\mathrm{m}^{3}$ air & $+/-\%$ & $\mathrm{~m}^{3}$ water & $+/-\%$ & cm242 & 1.837 \\
\hline Charge & $1.487 \mathrm{E}+19$ & $\mathrm{~N} / \mathrm{A}$ & $2.809 \mathrm{E}+12$ & $\mathrm{~N} / \mathrm{A}$ & cm244 & 16.925 \\
\hline Discharge & $2.039 \mathrm{E}+19$ & $\mathrm{~N} / \mathrm{A}$ & $5.674 \mathrm{E}+12$ & $\mathrm{~N} / \mathrm{A}$ & cm245 & 9.295 \\
\hline $1 \mathrm{yr}$ & $1.689 \mathrm{E}+19$ & $\mathrm{~N} / \mathrm{A}$ & $3.633 \mathrm{E}+12$ & $\mathrm{~N} / \mathrm{A}$ & \multicolumn{2}{|c|}{ FP (+/- \%) } \\
\hline $5 \mathrm{yr}$ & $1.571 \mathrm{E}+19$ & 11.560 & $3.293 \mathrm{E}+12$ & 10.454 & c 14 & 0.962 \\
\hline $10 \mathrm{yr}$ & $1.465 E+19$ & 10.447 & $3.025 \mathrm{E}+12$ & 9.585 & se 79 & 0.627 \\
\hline $50 \mathrm{yr}$ & $9.804 \mathrm{E}+18$ & 5.515 & $1.946 \mathrm{E}+12$ & 5.256 & sr 90 & 0.180 \\
\hline $100 \mathrm{yr}$ & $7.387 \mathrm{E}+18$ & 4.353 & $1.422 \mathrm{E}+12$ & 4.273 & tc 99 & 0.472 \\
\hline $500 \mathrm{yr}$ & $2.967 \mathrm{E}+18$ & 2.627 & $5.580 \mathrm{E}+11$ & 2.627 & i129 & 0.460 \\
\hline $1000 \mathrm{yr}$ & $2.003 E+18$ & 2.559 & $3.765 \mathrm{E}+11$ & 2.558 & cs137 & 0.098 \\
\hline $2500 \mathrm{yr}$ & $1.293 E+18$ & 2.922 & $2.431 \mathrm{E}+11$ & 2.920 & ba137m & 0.092 \\
\hline $5000 \mathrm{yr}$ & $1.019 \mathrm{E}+18$ & 2.998 & $1.916 \mathrm{E}+11$ & 2.996 & y90 & 0.181 \\
\hline $10000 \mathrm{yr}$ & $7.050 \mathrm{E}+17$ & 2.992 & $1.325 \mathrm{E}+11$ & 2.991 & cs134 & 2.250 \\
\hline \multirow{2}{*}{\multicolumn{5}{|c|}{$\begin{array}{l}\text { Main Contributors to Uncertainty in } \\
\text { Decay Heat and Radioactivity }\end{array}$}} & \multicolumn{2}{|c|}{ k-effective Values } \\
\hline & & & & & BOL & 1.1779 \\
\hline & \multicolumn{2}{|c|}{ Heat } & \multicolumn{2}{|c|}{ Activity } & EOL & 1.1195 \\
\hline $5 \mathrm{yr}$ & \multicolumn{2}{|c|}{$\mathrm{Cm}-244$} & \multicolumn{2}{|c|}{$\mathrm{Cm}-244, \mathrm{Pu}-241$} & & \\
\hline $10 \mathrm{yr}$ & \multicolumn{2}{|c|}{$\mathrm{Cm}-244$} & \multicolumn{2}{|c|}{ Cm-244, Pu-241 } & & \\
\hline $50 \mathrm{yr}$ & \multicolumn{2}{|c|}{$\mathrm{Cm}-244, \mathrm{Pu}-238$} & \multicolumn{2}{|c|}{$\begin{array}{c}\mathrm{Cm}-244, \mathrm{Pu}-238, \mathrm{Pu}- \\
241\end{array}$} & & \\
\hline $100 \mathrm{yr}$ & \multicolumn{2}{|c|}{ Pu-238, Am-241 } & \multicolumn{2}{|c|}{ Pu-238, Am-241 } & & \\
\hline $500 \mathrm{yr}$ & \multicolumn{2}{|c|}{ Am-241 } & \multicolumn{2}{|c|}{ Am-241 } & & \\
\hline $1000 \mathrm{yr}$ & \multicolumn{2}{|c|}{ Pu-240, Am-241 } & $\mathrm{Pu}-240, \mathrm{~A}$ & -241 & & \\
\hline $2500 \mathrm{yr}$ & Pu-239, F & $1-240$ & $\mathrm{Pu}-239, \mathrm{~F}$ & -240 & & \\
\hline $5000 \mathrm{yr}$ & Pu-239, & $1-240$ & Pu-239, F & -240 & & \\
\hline $10000 \mathrm{yr}$ & Pu-239, & $1-240$ & Pu-239, F & -240 & & \\
\hline
\end{tabular}

Table C.12: Results table for fast reactor fuel of $\mathrm{CR}=\mathbf{0 . 7 0}$. 


\begin{tabular}{|c|c|c|c|c|c|c|}
\hline \multicolumn{7}{|c|}{ Results Table for FR, CR=1. } \\
\hline \multicolumn{5}{|c|}{ Uncertainty for Key Metrics } & \multicolumn{2}{|c|}{$\begin{array}{l}\text { Isotopic Mass } \\
\text { Uncertainties }\end{array}$} \\
\hline & \multicolumn{2}{|c|}{ Heat } & \multicolumn{2}{|c|}{ Activity } & \multicolumn{2}{|c|}{ Actindes (+/- \%) } \\
\hline & $\mathbf{W}$ & $+/-\%$ & $\mathbf{C i}$ & $+/-\%$ & u235 & 3.267 \\
\hline Charge & $1.632 \mathrm{E}+03$ & $\mathrm{~N} / \mathrm{A}$ & $3.224 \mathrm{E}+05$ & $\mathrm{~N} / \mathrm{A}$ & u236 & 5.535 \\
\hline Discharge & $1.024 \mathrm{E}+05$ & $\mathrm{~N} / \mathrm{A}$ & $3.537 \mathrm{E}+07$ & $\mathrm{~N} / \mathrm{A}$ & u237 & 4.247 \\
\hline $1 \mathrm{yr}$ & $6.572 \mathrm{E}+03$ & $\mathrm{~N} / \mathrm{A}$ & $1.415 \mathrm{E}+06$ & $\mathrm{~N} / \mathrm{A}$ & u238 & 0.255 \\
\hline $5 \mathrm{yr}$ & $4.281 \mathrm{E}+03$ & 6.259 & $1.149 \mathrm{E}+06$ & 3.371 & np237 & 2.828 \\
\hline $10 \mathrm{yr}$ & $3.682 \mathrm{E}+03$ & 6.126 & $9.487 \mathrm{E}+05$ & 3.213 & np239 & 15.989 \\
\hline $50 \mathrm{yr}$ & $2.403 \mathrm{E}+03$ & 3.672 & $3.106 \mathrm{E}+05$ & 1.628 & pu238 & 5.204 \\
\hline $100 \mathrm{yr}$ & $1.735 \mathrm{E}+03$ & 3.634 & $1.156 \mathrm{E}+05$ & 1.685 & pu239 & 2.800 \\
\hline $500 \mathrm{yr}$ & $8.261 \mathrm{E}+02$ & 3.638 & $2.591 \mathrm{E}+04$ & 3.572 & pu240 & 3.066 \\
\hline $1000 \mathrm{yr}$ & $5.835 \mathrm{E}+02$ & 3.513 & $1.852 \mathrm{E}+04$ & 3.496 & pu241 & 4.246 \\
\hline $2500 \mathrm{yr}$ & $3.873 \mathrm{E}+02$ & 3.989 & $1.253 \mathrm{E}+04$ & 3.966 & pu242 & 3.141 \\
\hline $5000 \mathrm{yr}$ & $3.117 \mathrm{E}+02$ & 4.060 & $1.011 \mathrm{E}+04$ & 4.025 & am241 & 3.271 \\
\hline \multirow[t]{3}{*}{$10000 \mathrm{yr}$} & $2.240 \mathrm{E}+02$ & 4.014 & $7.275 \mathrm{E}+03$ & 3.977 & am242m & 1.972 \\
\hline & \multicolumn{2}{|c|}{ Inhalation Hazard } & \multicolumn{2}{|c|}{ Ingestion Hazard } & am243 & 15.989 \\
\hline & $\mathrm{m}^{3}$ air & $+/-\%$ & $\mathrm{~m}^{3}$ water & $+/-\%$ & cm242 & 1.972 \\
\hline Charge & $3.353 E+18$ & N/A & $6.323 \mathrm{E}+11$ & $\mathrm{~N} / \mathrm{A}$ & cm244 & 19.875 \\
\hline Discharge & $5.606 \mathrm{E}+18$ & $\mathrm{~N} / \mathrm{A}$ & $3.066 \mathrm{E}+12$ & $\mathrm{~N} / \mathrm{A}$ & cm245 & 11.948 \\
\hline $1 \mathrm{yr}$ & $4.657 \mathrm{E}+18$ & $\mathrm{~N} / \mathrm{A}$ & $1.334 \mathrm{E}+12$ & $\mathrm{~N} / \mathrm{A}$ & \multicolumn{2}{|c|}{ FP (+/- \%) } \\
\hline 5 yr & $4.466 \mathrm{E}+18$ & 6.841 & 1.169E+12 & 4.951 & c 14 & 0.708 \\
\hline $10 \mathrm{yr}$ & $4.339 \mathrm{E}+18$ & 6.173 & 1.076E+12 & 4.714 & se 79 & 0.544 \\
\hline $50 \mathrm{yr}$ & $3.603 E+18$ & 4.224 & $7.747 \mathrm{E}+11$ & 3.707 & sr 90 & 0.159 \\
\hline $100 \mathrm{yr}$ & $3.061 \mathrm{E}+18$ & 3.878 & $6.055 \mathrm{E}+11$ & 3.695 & tc 99 & 0.554 \\
\hline $500 \mathrm{yr}$ & $1.681 \mathrm{E}+18$ & 3.508 & $3.159 \mathrm{E}+11$ & 3.507 & i129 & 0.494 \\
\hline $1000 \mathrm{yr}$ & $1.247 \mathrm{E}+18$ & 3.560 & $2.344 \mathrm{E}+11$ & 3.559 & cs137 & 0.099 \\
\hline $2500 \mathrm{yr}$ & $8.838 \mathrm{E}+17$ & 4.036 & $1.661 \mathrm{E}+11$ & 4.035 & ba137m & 0.099 \\
\hline $5000 \mathrm{yr}$ & $7.181 \mathrm{E}+17$ & 4.073 & $1.349 \mathrm{E}+11$ & 4.072 & y90 & 0.158 \\
\hline $10000 \mathrm{yr}$ & $5.161 \mathrm{E}+17$ & 4.029 & $9.696 \mathrm{E}+10$ & 4.028 & $\operatorname{cs134}$ & 2.214 \\
\hline \multirow{2}{*}{\multicolumn{5}{|c|}{$\begin{array}{l}\text { Main Contributors to Uncertainty in } \\
\text { Decay Heat and Radioactivity }\end{array}$}} & \multicolumn{2}{|c|}{ k-effective Values } \\
\hline & & & & & BOL & 1.0234 \\
\hline & \multicolumn{2}{|c|}{ Heat } & \multicolumn{2}{|c|}{ Activity } & EOL & 1.0452 \\
\hline $5 \mathrm{yr}$ & \multicolumn{2}{|c|}{$\mathrm{Cm}-244$} & \multicolumn{2}{|c|}{$\mathrm{Cm}-244, \mathrm{Pu}-241$} & & \\
\hline $10 \mathrm{yr}$ & \multicolumn{2}{|c|}{$\mathrm{Cm}-244$} & \multicolumn{2}{|c|}{ Cm-244, Pu-241 } & & \\
\hline $50 \mathrm{yr}$ & \multicolumn{2}{|c|}{$\mathrm{Cm}-244, \mathrm{Pu}-238$} & \multicolumn{2}{|c|}{$\begin{array}{c}\mathrm{Cm}-244, \mathrm{Pu}-238, \mathrm{Pu}- \\
241\end{array}$} & & \\
\hline $100 \mathrm{yr}$ & \multicolumn{2}{|c|}{ Pu-238, Am-241 } & \multicolumn{2}{|c|}{ Pu-238, Am-241 } & & \\
\hline $500 \mathrm{yr}$ & \multicolumn{2}{|c|}{ Pu-240, Am-241 } & \multicolumn{2}{|c|}{ Pu-240,Am-241 } & & \\
\hline $1000 \mathrm{yr}$ & \multicolumn{2}{|c|}{$\begin{array}{l}\text { Pu-238, Pu-240, Am- } \\
241\end{array}$} & $\begin{array}{r}\mathrm{Pu}-239, \mathrm{Pu}-2 \\
241\end{array}$ & 40, Am- & & \\
\hline $2500 \mathrm{yr}$ & $\mathrm{Pu}-239, \mathrm{P}$ & -240 & $\mathrm{Pu}-239, \mathrm{P}$ & -240 & & \\
\hline $5000 \mathrm{yr}$ & $\mathrm{Pu}-239, \mathrm{P}$ & -240 & $\mathrm{Pu}-239, \mathrm{P}$ & -240 & & \\
\hline $10000 \mathrm{yr}$ & $\mathrm{Pu}-239, \mathrm{P}$ & -240 & $\mathrm{Pu}-239, \mathrm{P}$ & -240 & & \\
\hline
\end{tabular}

Table C.13: Results table for fast reactor fuel of $\mathrm{CR}=\mathbf{1 . 0 5}$. 


\begin{tabular}{|c|c|c|c|c|c|c|}
\hline \multicolumn{7}{|c|}{ Results Table for Recycled FR, CR=0.7, burned 41.4 GWD/MTU } \\
\hline \multicolumn{5}{|c|}{ Uncertainty for Key Metrics } & \multicolumn{2}{|c|}{$\begin{array}{c}\text { Isotopic Mass } \\
\text { Uncertainties }\end{array}$} \\
\hline & \multicolumn{2}{|c|}{ Heat } & \multicolumn{2}{|c|}{ Activity } & \multicolumn{2}{|c|}{ Actindes (+/- \%) } \\
\hline & $\mathbf{W}$ & $+/-\%$ & $\mathbf{C i}$ & $+/-\%$ & u235 & 2.819 \\
\hline Charge & $9.086 \mathrm{E}+03$ & $\mathrm{~N} / \mathrm{A}$ & $1.138 \mathrm{E}+06$ & N/A & u236 & 5.757 \\
\hline Discharge & $1.331 \mathrm{E}+05$ & $\mathrm{~N} / \mathrm{A}$ & $3.272 \mathrm{E}+07$ & $\mathrm{~N} / \mathrm{A}$ & u237 & 6.671 \\
\hline $1 \mathrm{yr}$ & $1.770 \mathrm{E}+04$ & $\mathrm{~N} / \mathrm{A}$ & $2.020 \mathrm{E}+06$ & $\mathrm{~N} / \mathrm{A}$ & u238 & 0.260 \\
\hline $5 \mathrm{yr}$ & $1.220 \mathrm{E}+04$ & 19.983 & $1.640 \mathrm{E}+06$ & 8.875 & np237 & 11.435 \\
\hline $10 \mathrm{yr}$ & $1.100 \mathrm{E}+04$ & 18.698 & $1.356 \mathrm{E}+06$ & 8.585 & np239 & 46.662 \\
\hline $50 \mathrm{yr}$ & $6.348 \mathrm{E}+03$ & 11.953 & $4.069 \mathrm{E}+05$ & 6.594 & pu238 & 12.869 \\
\hline $100 \mathrm{yr}$ & $4.433 \mathrm{E}+03$ & 10.818 & $1.790 \mathrm{E}+05$ & 8.118 & pu239 & 4.924 \\
\hline $500 \mathrm{yr}$ & $1.765 \mathrm{E}+03$ & 8.443 & $5.555 \mathrm{E}+04$ & 8.215 & pu240 & 5.482 \\
\hline $1000 \mathrm{yr}$ & $1.126 \mathrm{E}+03$ & 7.500 & $3.599 \mathrm{E}+04$ & 7.452 & pu241 & 6.671 \\
\hline $2500 \mathrm{yr}$ & $6.560 \mathrm{E}+02$ & 7.838 & $2.160 \mathrm{E}+04$ & 7.966 & pu242 & 6.429 \\
\hline $5000 \mathrm{yr}$ & $5.044 \mathrm{E}+02$ & 7.979 & $1.669 \mathrm{E}+04$ & 8.082 & am241 & 7.244 \\
\hline \multirow[t]{3}{*}{$10000 \mathrm{yr}$} & $3.430 \mathrm{E}+02$ & 7.500 & $1.134 \mathrm{E}+04$ & 7.591 & am242m & 9.284 \\
\hline & \multicolumn{2}{|c|}{ Inhalation Hazard } & \multicolumn{2}{|c|}{ Ingestion Hazard } & am243 & 27.480 \\
\hline & $\mathrm{m}^{3}$ air & $+/-\%$ & $\mathrm{~m}^{3}$ water & $+/-\%$ & cm242 & 9.284 \\
\hline Charge & $1.487 \mathrm{E}+19$ & $\mathrm{~N} / \mathrm{A}$ & $2.809 \mathrm{E}+12$ & N/A & cm244 & 19.265 \\
\hline Discharge & $2.265 \mathrm{E}+19$ & $\mathrm{~N} / \mathrm{A}$ & $5.779 \mathrm{E}+12$ & $\mathrm{~N} / \mathrm{A}$ & cm245 & 15.308 \\
\hline $1 \mathrm{yr}$ & $1.866 \mathrm{E}+19$ & $\mathrm{~N} / \mathrm{A}$ & $3.736 \mathrm{E}+12$ & $\mathrm{~N} / \mathrm{A}$ & \multicolumn{2}{|c|}{ FP (+/- \%) } \\
\hline $5 \mathrm{yr}$ & $1.733 \mathrm{E}+19$ & 16.097 & $3.442 \mathrm{E}+12$ & 15.353 & c 14 & 3.042 \\
\hline $10 \mathrm{yr}$ & $1.615 \mathrm{E}+19$ & 15.176 & $3.188 \mathrm{E}+12$ & 14.555 & se 79 & 1.548 \\
\hline $50 \mathrm{yr}$ & $1.081 \mathrm{E}+19$ & 12.097 & $2.092 \mathrm{E}+12$ & 11.819 & sr 90 & 0.410 \\
\hline $100 \mathrm{yr}$ & $8.227 \mathrm{E}+18$ & 11.132 & $1.566 \mathrm{E}+12$ & 11.042 & tc 99 & 0.514 \\
\hline $500 \mathrm{yr}$ & $3.474 \mathrm{E}+18$ & 7.866 & $6.533 \mathrm{E}+11$ & 7.866 & i129 & 0.854 \\
\hline $1000 \mathrm{yr}$ & $2.333 E+18$ & 7.262 & $4.385 \mathrm{E}+11$ & 7.261 & cs137 & 0.144 \\
\hline $2500 \mathrm{yr}$ & $1.475 \mathrm{E}+18$ & 7.865 & $2.772 \mathrm{E}+11$ & 7.861 & ba137m & 0.140 \\
\hline $5000 \mathrm{yr}$ & $1.150 \mathrm{E}+18$ & 7.919 & $2.161 \mathrm{E}+11$ & 7.915 & y90 & 0.410 \\
\hline $10000 \mathrm{yr}$ & $7.816 \mathrm{E}+17$ & 7.453 & $1.469 \mathrm{E}+11$ & 7.449 & cs134 & 2.966 \\
\hline \multirow{2}{*}{\multicolumn{5}{|c|}{$\begin{array}{l}\text { Main Contributors to Uncertainty in } \\
\text { Decay Heat and Radioactivity }\end{array}$}} & \multicolumn{2}{|c|}{ k-effective Values } \\
\hline & & & & & BOL & 1.1731 \\
\hline & \multicolumn{2}{|c|}{ Heat } & \multicolumn{2}{|c|}{ Activity } & EOL & 1.1468 \\
\hline $5 \mathrm{yr}$ & \multicolumn{2}{|c|}{$\mathrm{Cm}-244$} & \multicolumn{2}{|c|}{$\mathrm{Cm}-244, \mathrm{Pu}-241$} & & \\
\hline $10 \mathrm{yr}$ & \multicolumn{2}{|c|}{$\mathrm{Cm}-244$} & \multicolumn{2}{|c|}{ Cm-244, Pu-241 } & & \\
\hline $50 \mathrm{yr}$ & \multicolumn{2}{|c|}{$\mathrm{Cm}-244, \mathrm{Pu}-238$} & \multicolumn{2}{|c|}{$\begin{array}{c}\mathrm{Cm}-244, \mathrm{Pu}-238, \mathrm{Pu}- \\
241 \\
\end{array}$} & & \\
\hline $100 \mathrm{yr}$ & \multicolumn{2}{|c|}{ Pu-238, Am-241 } & \multicolumn{2}{|c|}{$\mathrm{Pu}-238, \mathrm{Am}-241$} & & \\
\hline $500 \mathrm{yr}$ & \multicolumn{2}{|c|}{ Pu-240, Am-241 } & \multicolumn{2}{|c|}{ Pu-240,Am-241 } & & \\
\hline $1000 \mathrm{yr}$ & \multicolumn{2}{|c|}{ Pu-239, Pu-240, Am-241 } & $\begin{array}{r}\mathrm{Pu}-239, \mathrm{Pu}- \\
241 \\
\end{array}$ & 40, Am- & & \\
\hline $2500 \mathrm{yr}$ & Pu-239 & $1-240$ & $\mathrm{Pu}-239, \mathrm{~F}$ & -240 & & \\
\hline $5000 \mathrm{yr}$ & Pu-239 & $1-240$ & $\mathrm{Pu}-239, \mathrm{~F}$ & $1-240$ & & \\
\hline $10000 \mathrm{yr}$ & Pu-239 & $1-240$ & $\mathrm{Pu}-239, \mathrm{~F}$ & -240 & & \\
\hline
\end{tabular}

Table C.14: Results table for equilibrium recycled fast reactor fuel of $\mathrm{CR}=0.70$. 


\begin{tabular}{|c|c|c|c|c|c|c|}
\hline \multicolumn{7}{|c|}{ Results Table for Recycled FR, } \\
\hline \multicolumn{5}{|c|}{ Uncertainty for Key Metrics } & \multirow{2}{*}{\multicolumn{2}{|c|}{$\begin{array}{c}\begin{array}{c}\text { Isotopic Mass } \\
\text { Uncertainties }\end{array} \\
\text { Actindes (+/- \%) }\end{array}$}} \\
\hline+2 & \multicolumn{2}{|c|}{ Heat } & \multicolumn{2}{|c|}{ Activity } & & \\
\hline & $\mathbf{W}$ & $+/-\%$ & $\mathbf{C i}$ & $+/-\%$ & u235 & 0.809 \\
\hline Charge & $3.474 \mathrm{E}+04$ & $\mathrm{~N} / \mathrm{A}$ & $1.806 \mathrm{E}+06$ & N/A & u236 & 2.034 \\
\hline Discharge & $3.871 \mathrm{E}+04$ & N/A & $1.941 \mathrm{E}+06$ & $\mathrm{~N} / \mathrm{A}$ & u237 & -- \\
\hline $1 \mathrm{yr}$ & $1.634 \mathrm{E}+04$ & $\mathrm{~N} / \mathrm{A}$ & $1.288 \mathrm{E}+06$ & $\mathrm{~N} / \mathrm{A}$ & u238 & 0.950 \\
\hline 5 yr & $9.725 \mathrm{E}+03$ & 22.005 & $9.632 \mathrm{E}+05$ & 15.819 & np237 & 7.344 \\
\hline $10 \mathrm{yr}$ & $8.980 \mathrm{E}+03$ & 20.959 & $7.979 \mathrm{E}+05$ & 15.327 & np239 & -- \\
\hline $50 \mathrm{yr}$ & $5.699 \mathrm{E}+03$ & 17.649 & $2.522 \mathrm{E}+05$ & 13.526 & pu238 & 18.616 \\
\hline $100 \mathrm{yr}$ & $4.192 \mathrm{E}+03$ & 16.693 & $1.374 \mathrm{E}+05$ & 15.399 & pu239 & 1.336 \\
\hline $500 \mathrm{yr}$ & $1.616 \mathrm{E}+03$ & 11.382 & $5.039 \mathrm{E}+04$ & 11.044 & pu240 & 7.463 \\
\hline $1000 \mathrm{yr}$ & $1.017 \mathrm{E}+03$ & 9.549 & $3.194 \mathrm{E}+04$ & 9.371 & pu241 & 10.570 \\
\hline $2500 \mathrm{yr}$ & $5.961 \mathrm{E}+02$ & 8.750 & $1.912 \mathrm{E}+04$ & 8.745 & pu242 & 18.769 \\
\hline $5000 \mathrm{yr}$ & $4.650 \mathrm{E}+02$ & 8.556 & $1.498 \mathrm{E}+04$ & 8.526 & am241 & 10.163 \\
\hline \multirow[t]{3}{*}{$10000 \mathrm{yr}$} & $3.258 \mathrm{E}+02$ & 7.269 & $1.050 \mathrm{E}+04$ & 7.241 & am242m & 14.836 \\
\hline & \multicolumn{2}{|c|}{ Inhalation Hazard } & \multicolumn{2}{|c|}{ Ingestion Hazard } & am243 & \\
\hline & $\mathrm{m}^{3}$ air & $+/-\%$ & $\mathrm{~m}^{3}$ water & $+/-\%$ & cm242 & 8.856 \\
\hline Charge & $1.779 \mathrm{E}+19$ & $\mathrm{~N} / \mathrm{A}$ & $3.348 \mathrm{E}+12$ & $\mathrm{~N} / \mathrm{A}$ & cm244 & 20.387 \\
\hline Discharge & $1.930 \mathrm{E}+19$ & $\mathrm{~N} / \mathrm{A}$ & $3.631 \mathrm{E}+12$ & $\mathrm{~N} / \mathrm{A}$ & cm245 & 38.508 \\
\hline $1 \mathrm{yr}$ & $1.712 \mathrm{E}+19$ & $\mathrm{~N} / \mathrm{A}$ & $3.230 \mathrm{E}+12$ & $\mathrm{~N} / \mathrm{A}$ & \multicolumn{2}{|c|}{ FP (+/- \%) } \\
\hline 5 yr & $1.575 \mathrm{E}+19$ & 19.334 & $2.974 \mathrm{E}+12$ & 19.369 & c 14 & -- \\
\hline $10 \mathrm{yr}$ & $1.482 \mathrm{E}+19$ & 19.027 & $2.797 \mathrm{E}+12$ & 19.062 & se 79 & -- \\
\hline $50 \mathrm{yr}$ & $1.040 \mathrm{E}+19$ & 18.267 & $1.962 E+12$ & 18.306 & sr 90 & -- \\
\hline $100 \mathrm{yr}$ & $7.993 \mathrm{E}+18$ & 16.936 & $1.506 \mathrm{E}+12$ & 16.977 & tc 99 & -- \\
\hline $500 \mathrm{yr}$ & $3.182 \mathrm{E}+18$ & 10.477 & $5.984 \mathrm{E}+11$ & 10.479 & i129 & -- \\
\hline $1000 \mathrm{yr}$ & $2.109 \mathrm{E}+18$ & 9.062 & $3.964 \mathrm{E}+11$ & 9.061 & cs137 & -- \\
\hline $2500 \mathrm{yr}$ & $1.342 \mathrm{E}+18$ & 8.907 & $2.522 \mathrm{E}+11$ & 8.902 & ba137m & -- \\
\hline $5000 \mathrm{yr}$ & $1.061 \mathrm{E}+18$ & 8.624 & $1.995 \mathrm{E}+11$ & 8.619 & y90 & -- \\
\hline $10000 \mathrm{yr}$ & $7.437 \mathrm{E}+17$ & 7.322 & $1.398 \mathrm{E}+11$ & 7.316 & cs134 & -- \\
\hline \multirow{2}{*}{\multicolumn{5}{|c|}{$\begin{array}{l}\text { Main Contributors to Uncertainty in } \\
\text { Decay Heat and Radioactivity }\end{array}$}} & \multicolumn{2}{|c|}{ k-Effective Values } \\
\hline & & & & & BOC & 1.00638 \\
\hline & \multicolumn{2}{|c|}{ Heat } & \multicolumn{2}{|c|}{ Activity } & EOC & 0.99925 \\
\hline $5 \mathrm{yr}$ & \multicolumn{2}{|c|}{$\mathrm{Pu}-238, \mathrm{Cm}-244$} & \multicolumn{2}{|c|}{ Cm-244, Pu-241 } & \multicolumn{2}{|c|}{ Conversion Ratio at EOC } \\
\hline $10 \mathrm{yr}$ & \multicolumn{2}{|c|}{ Cm-244, Pu-238 } & \multicolumn{2}{|c|}{$\begin{array}{c}\mathrm{Cm}-244, \mathrm{Pu}-238, \mathrm{Pu}- \\
241\end{array}$} & \multicolumn{2}{|c|}{0.7695} \\
\hline $50 \mathrm{yr}$ & \multicolumn{2}{|c|}{$\begin{array}{c}\text { Cm-244, Pu-238, } \\
\text { Am241 }\end{array}$} & \multicolumn{2}{|c|}{$\begin{array}{c}\text { Am-241, Pu-238, Pu- } \\
241 \\
\end{array}$} & & \\
\hline $100 \mathrm{yr}$ & \multicolumn{2}{|c|}{ Pu-238, Am-241 } & \multicolumn{2}{|c|}{ Pu-238, Am-241 } & & \\
\hline $500 \mathrm{yr}$ & \multicolumn{2}{|c|}{ Pu-240, Am-241 } & \multicolumn{2}{|c|}{ Pu-240,Am-241 } & & \\
\hline $1000 \mathrm{yr}$ & $\mathrm{Pu}-240, \mathrm{Ar}$ & -241 & $\mathrm{Pu}-240, \mathrm{Ar}$ & ר-241 & & \\
\hline $2500 \mathrm{yr}$ & $\mathrm{Pu}-239, \mathrm{P}$ & $1-240$ & $\mathrm{Pu}-239, \mathrm{P}$ & $1-240$ & & \\
\hline 5000 yr & $\mathrm{Pu}-239, \mathrm{P}$ & $1-240$ & $\mathrm{Pu}-239, \mathrm{P}$ & $1-240$ & & \\
\hline $10000 \mathrm{yr}$ & $\mathrm{Pu}-239, \mathrm{P}$ & $1-240$ & $\mathrm{Pu}-239, \mathrm{P}$ & $1-240$ & & \\
\hline
\end{tabular}

Table C.15: Results table for REBUS equilibrium recycled fast reactor fuel. 